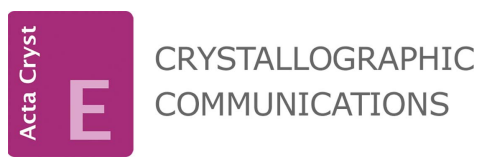

ISSN 2056-9890
Received 5 August 2019

Accepted 11 September 2019

Edited by J. Ellena, Universidade de Sâo Paulo, Brazil

Keywords: synthesis; piperazines; crystal structure; disorder; twinning; hydrogen bonding; supramolecular assembly.

CCDC references: $1953078 ; 1953077$; 1953076; 1953075; 1953074; 1953073; $1953072 ; 1953071 ; 1953070 ; 1953069$; $1953068 ; 1953067$

Supporting information: this article has supporting information at journals.iucr.org/e

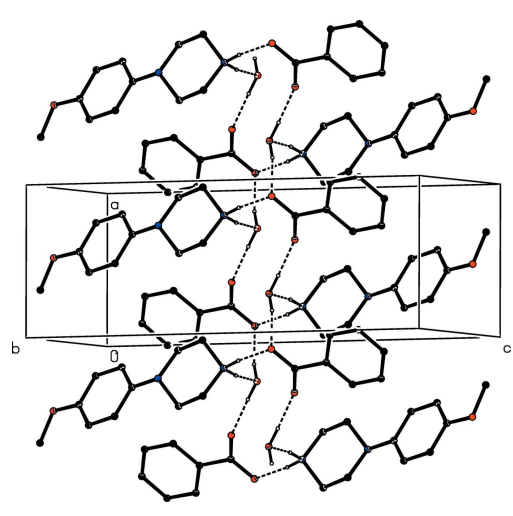

OPEN $\partial$ ACCESS

\section{Twelve 4-(4-methoxyphenyl)piperazin-1-ium salts containing organic anions: supramolecular assembly in one, two and three dimensions}

\author{
Haruvegowda Kiran Kumar, ${ }^{a}$ Hemmige S. Yathirajan, ${ }^{a *}$ Sabine Foro ${ }^{\mathrm{b}}$ and \\ Christopher Glidewell
}

\begin{abstract}
${ }^{\mathbf{a}}$ Department of Studies in Chemistry, University of Mysore, Manasagangotri, Mysuru-570 006, India, ${ }^{\mathbf{b}}$ Institute of Materials Science, Darmstadt University of Technology, Petersenstrasse 23, D-64287 Darmstadt, Germany, and ${ }^{\mathbf{c}}$ School of Chemistry, University of St Andrews, St Andrews, Fife KY16 9ST, UK. *Correspondence e-mail: yathirajan@hotmail.com
\end{abstract}

Twelve 4-(4-methoxyphenyl)piperazin-1-ium salts containing organic anions have been prepared and structurally characterized. The monohydrated benzoate, 4-fluorobenzoate, 4-chlorobenzoate and 4-bromobenzoate salts, $\mathrm{C}_{11} \mathrm{H}_{17} \mathrm{~N}_{2} \mathrm{O}^{+} \cdot \mathrm{C}_{7} \mathrm{H}_{5} \mathrm{O}_{2}^{-} \cdot \mathrm{H}_{2} \mathrm{O} \quad$ (I), $\quad \mathrm{C}_{11} \mathrm{H}_{17} \mathrm{~N}_{2} \mathrm{O}^{+} \cdot \mathrm{C}_{7} \mathrm{H}_{4} \mathrm{FO}_{2}{ }^{-} \cdot \mathrm{H}_{2} \mathrm{O} \quad$ (II), $\mathrm{C}_{11} \mathrm{H}_{17} \mathrm{~N}_{2} \mathrm{O}^{+} \cdot \mathrm{C}_{7} \mathrm{H}_{4} \mathrm{ClO}_{2}{ }^{-} \cdot \mathrm{H}_{2} \mathrm{O}$ (III), and $\mathrm{C}_{11} \mathrm{H}_{17} \mathrm{~N}_{2} \mathrm{O}^{+} \cdot \mathrm{C}_{7} \mathrm{H}_{4} \mathrm{BrO}_{2}{ }^{-} \cdot \mathrm{H}_{2} \mathrm{O}$ (IV), respectively, are isomorphous and all exhibit disorder in the 4-methoxyphenyl unit: the components are linked by $\mathrm{N}-\mathrm{H} \cdots \mathrm{O}$ and $\mathrm{O}-\mathrm{H} \cdots \mathrm{O}$ hydrogen bond to form chains of rings. The unsolvated 2-hydroxybenzoate, pyridine-3-carboxylate and 2-hydroxy-3,5-dinitrobenzoate salts, $\mathrm{C}_{11} \mathrm{H}_{17} \mathrm{~N}_{2} \mathrm{O}^{+} \cdot \mathrm{C}_{7} \mathrm{H}_{5} \mathrm{O}_{3}{ }^{-}$(V), $\mathrm{C}_{11} \mathrm{H}_{17} \mathrm{~N}_{2} \mathrm{O}^{+} \cdot \mathrm{C}_{6} \mathrm{H}_{4} \mathrm{NO}_{2}{ }^{-}$(VI) and $\mathrm{C}_{11} \mathrm{H}_{17} \mathrm{~N}_{2} \mathrm{O}^{+} \cdot \mathrm{C}_{7} \mathrm{H}_{3} \mathrm{~N}_{2} \mathrm{O}_{7}^{-}$(VII), respectively, are all fully ordered: the components of $(\mathrm{V})$ are linked by multiple $\mathrm{N}-\mathrm{H} \cdots \mathrm{O}$ hydrogen bonds to form a chain of rings; those of (VI) are linked into a threedimensional framework by a combination of $\mathrm{N}-\mathrm{H} \cdots \mathrm{O}, \mathrm{C}-\mathrm{H} \cdots \mathrm{O}$ and $\mathrm{C}-$ $\mathrm{H} \cdots \mathrm{N}$ hydrogen bonds and those of (VII), where the anion has a structure reminiscent of the picrate anion, are linked into a three-dimensional array by $\mathrm{N}-\mathrm{H} \cdots \mathrm{O}$ and $\mathrm{C}-\mathrm{H} \cdots \mathrm{O}$ hydrogen bonds. The hydrogensuccinate and hydrogenfumarate salts, $\mathrm{C}_{11} \mathrm{H}_{17} \mathrm{~N}_{2} \mathrm{O}^{+} \cdot \mathrm{C}_{4} \mathrm{H}_{5} \mathrm{O}_{4}{ }^{-}$(VIII) and $\mathrm{C}_{11} \mathrm{H}_{17} \mathrm{~N}_{2} \mathrm{O}^{+}$.$\mathrm{C}_{4} \mathrm{H}_{3} \mathrm{O}_{3}{ }^{-}$(IX), respectively, are isomorphous, and both exhibit disorder in the anionic component: $\mathrm{N}-\mathrm{H} \cdots \mathrm{O}$ and $\mathrm{O}-\mathrm{H} \cdots \mathrm{O}$ hydrogen bonds link the ions into sheets, which are further linked by $\mathrm{C}-\mathrm{H} \cdots \pi($ arene) interactions. The anion of the hydrogenmaleate salt, $\mathrm{C}_{11} \mathrm{H}_{17} \mathrm{~N}_{2} \mathrm{O}^{+} \cdot \mathrm{C}_{4} \mathrm{H}_{3} \mathrm{O}_{3}{ }^{-}(\mathrm{X})$, contains a very short and nearly symmetrical $\mathrm{O} \cdots \mathrm{H} \cdots \mathrm{O}$ hydrogen bond, and $\mathrm{N}-\mathrm{H} \cdots \mathrm{O}$ hydrogen bonds link the anions into chains of rings. The ions in the trichloroacetate salt, $\mathrm{C}_{11} \mathrm{H}_{17} \mathrm{~N}_{2} \mathrm{O}^{+} \cdot \mathrm{C}_{2} \mathrm{Cl}_{3} \mathrm{O}_{2}{ }^{-}$(XI), are linked into simple chains by $\mathrm{N}-\mathrm{H} \cdots \mathrm{O}$ hydrogen bonds. In the hydrated chloranilate salt, $2 \mathrm{C}_{11} \mathrm{H}_{17} \mathrm{~N}_{2} \mathrm{O}^{+} \cdot \mathrm{C}_{6} \mathrm{Cl}_{2} \mathrm{O}_{4}{ }^{2-} \cdot 2 \mathrm{H}_{2} \mathrm{O}$ (XII), which crystallizes as a non-merohedral twin, the anion lies across a centre of inversion in space group $P 2_{1} / n$, and a combination of $\mathrm{N}-\mathrm{H} \cdots \mathrm{O}$ and $\mathrm{O}-\mathrm{H} \cdots \mathrm{O}$ hydrogen bonds generates complex sheets. Comparisons are made with the structures of some related compounds.

\section{Chemical context}

In recent years, $N$-(4-methoxyphenyl)piperazine (MeOPP) has emerged as a new addition to the range of designer recreational drugs, and considerable effort has been invested in the development of methods for the detection both of MeOPP itself and of its metabolites $N$-(4-hydroxyphenyl)piperazine and 4-hydroxyaniline (Arbo et al., 2012) in human fluids (Staack \& Maurer, 2003; Staack et al., 2004). MeOPP has euphoric stimulant properties and its action on human physiology is similar to that of amphetamines (Staack 
\& Maurer, 2005; Wohlfarth et al., 2010), but it has a significantly lower potential for abuse (Nagai et al., 2007). However, no therapeutic applications of MeOPP have been reported to date. In view of the reported properties of MeOPP, coupled with the broad range of biological activities exhibited by piperazine derivatives (Asif, 2015; Brito et al., 2019), we have recently initiated a programme of study centred on $\mathrm{N}$-(4methoxyphenyl)piperazine derivatives, and we have recently reported the synthesis and structures of a series of 1-aroyl-4(4-methoxyphenyl)piperazines (Kiran Kumar et al., 2019). In a continuation of that work, we have now prepared a series of 4methoxyphenyl)piperazin-1-ium salts of simple organic acids, (I)-(XII), in order to study the various patterns of hydrogenbonding interactions present in these salts, which may eventually be of value in pharmacological and pharmaceutical applications (Kavitha et al., 2014; Kaur et al., 2015; Shaibah, Yathirajan et al., 2017; Shaibah, Sagar et al., 2017; Shaibah et al., 2019). Salts of this type are readily prepared by co-crystallizations of the piperazine and the acids in methanol solution and, in total, 28 different acids representing a wide range of chemical types were investigated (see Section 5): however, only twelve of these provided crystals suitable for singlecrystal X-ray diffraction, and thus we report here the molecular and supramolecular structures of (I)-(XII) (Figs. 1-12).<smiles></smiles><smiles>O=C(Br)Br</smiles>
$n\left(\mathrm{H}_{2} \mathrm{O}\right)$

(II) $\mathrm{Ar}=4-\mathrm{FC}_{6} \mathrm{H}_{4}, \quad n=1$

(III) $\mathrm{Ar}=4-\mathrm{ClC}_{6} \mathrm{H}_{4}, \quad n=$

(IV) $\mathrm{Ar}=4-\mathrm{BrC}_{6} \mathrm{H}_{4}, \quad n=$

(V) $\mathrm{Ar}=2-\mathrm{HOC}_{6} \mathrm{H}_{4}, \quad n=\mathrm{ni}$

(VI) $\mathrm{Ar}=3-\mathrm{C}_{5} \mathrm{H}_{4} \mathrm{~N}, \quad n=$ nil<smiles>COc1ccc(N2CC[NH+]3CC[NH+]2C3)cc1</smiles><smiles></smiles><smiles>[X][NH+]1CCN(c2ccc(OC)cc2)CC1</smiles>

(VIII) $X=$ hydrogensuccinate

(IX) $X=$ hydrogenfumarate

(X) $X=$ hydrogenmaleate

(XI) $X=$ trichloroacetate<smiles>COc1ccc(N2CC[NH+](C)CC2)cc1</smiles><smiles>O=c1c(Cl)c(Cl)c(=O)c(=O)c1=O</smiles>

(XII)

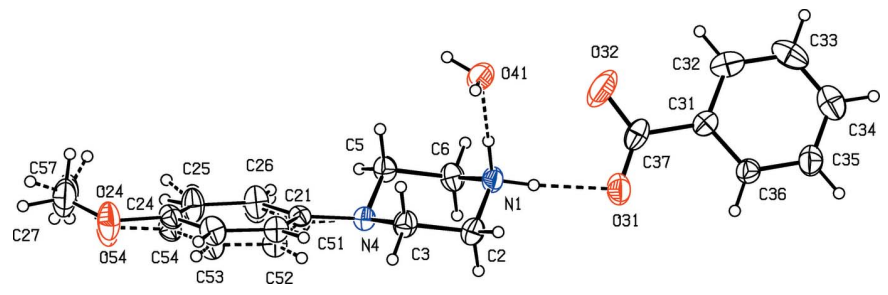

Figure 1

The independent components of compound (I), showing the atomlabelling scheme, the disorder of the 4-methoxyphenyl group, and the hydrogen bonds within the selected asymmetric unit. The major disorder component is drawn using full lines and the minor disorder component is drawn using dashed lines. Displacement ellipsoids are drawn at the $30 \%$ probability level and, for the sake of clarity, a few of the atom labels have been omitted.

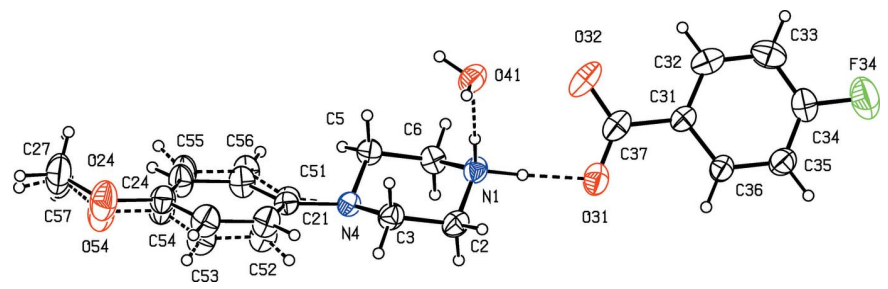

Figure 2

The independent components of compound (II), showing the atomlabelling scheme, the disorder of the 4-methoxyphenyl group, and the hydrogen bonds within the selected asymmetric unit. The major disorder component is drawn using full lines and the minor disorder component is drawn using dashed lines. Displacement ellipsoids are drawn at the $30 \%$ probability level and, for the sake of clarity, a few of the atom labels have been omitted.

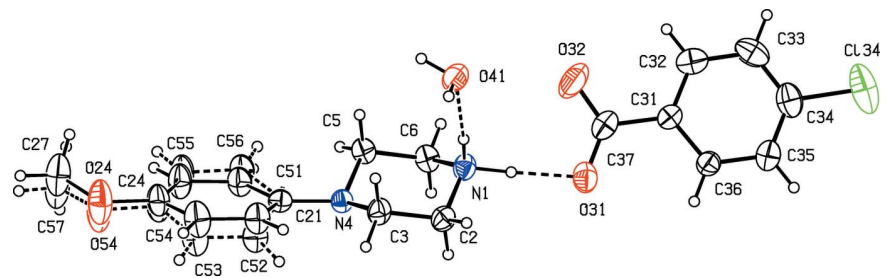

Figure 3

The independent components of compound (III), showing the atomlabelling scheme, the disorder of the 4-methoxyphenyl group, and the hydrogen bonds within the selected asymmetric unit. The major disorder component is drawn using full lines and the minor disorder component is drawn using dashed lines. Displacement ellipsoids are drawn at the $30 \%$ probability level and, for the sake of clarity, a few of the atom labels have been omitted.

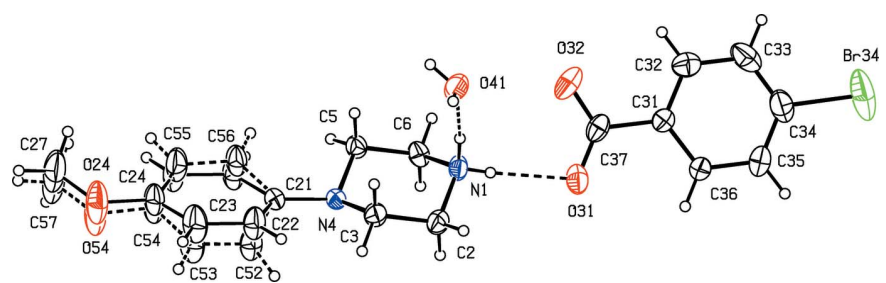

Figure 4

The independent components of compound (IV), showing the atomlabelling scheme, the disorder of the 4-methoxyphenyl group, and the hydrogen bonds within the selected asymmetric unit. The major disorder component is drawn using full lines and the minor disorder component is drawn dashed broken lines. Displacement ellipsoids are drawn at the $30 \%$ probability level and, for the sake of clarity, a few of the atom labels have been omitted. 


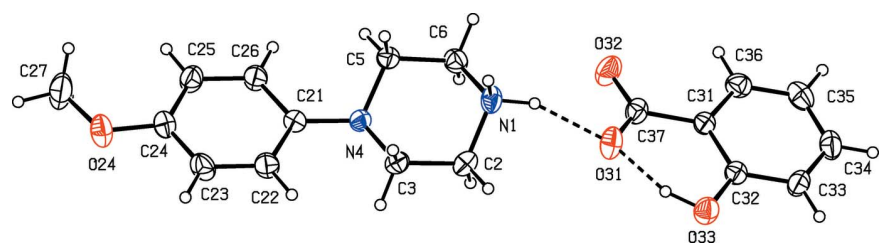

Figure 5

The independent components of compound (V), showing the atomlabelling scheme and the hydrogen bonds within the selected asymmetric unit. Displacement ellipsoids are drawn at the $30 \%$ probability level.

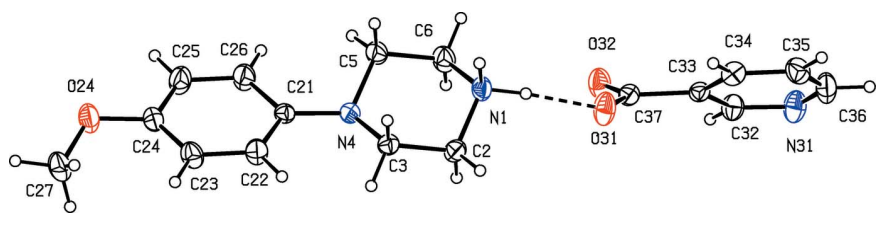

Figure 6

The independent components of compound (VI), showing the atomlabelling scheme and the hydrogen bonds within the selected asymmetric unit. Displacement ellipsoids are drawn at the $30 \%$ probability level.

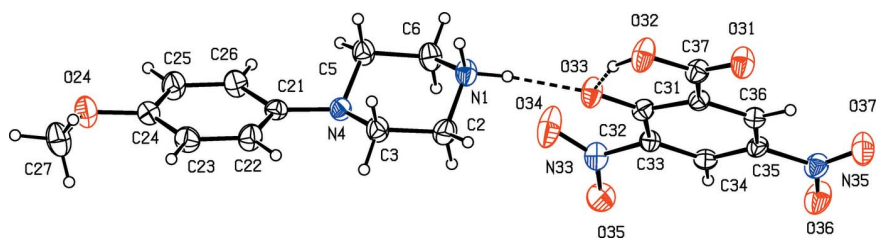

Figure 7

The independent components of compound (VII), showing the atomlabelling scheme and the hydrogen bond within the selected asymmetric unit. Displacement ellipsoids are drawn at the $30 \%$ probability level.

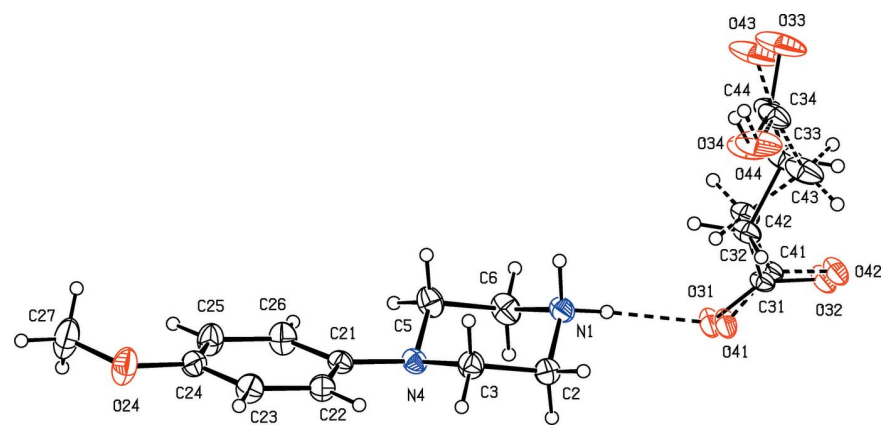

Figure 8

The independent components of compound (VIII), showing the atomlabelling scheme, the disorder of anion, and the hydrogen bonds within the selected asymmetric unit. The major disorder component is drawn using full lines and the minor disorder component is drawn using dashed lines. Displacement ellipsoids are drawn at the $30 \%$ probability level.

\section{Structural commentary}

Compounds (I)-(XI) are all 1:1 salts, but in (XII), where the dianion lies across a centre of inversion while the cation lies in a general position, the cation:anion ratio is 2:1. Compounds (I)-(IV) and (XII) all crystallize as hydrates, but compounds (V)-(XI) all crystallize in solvent-free form. Compounds (I)(IV) are isomorphous (Table 2), in each of which the 4-methoxyphenyl groups is disordered over two sets of atomic sites (Figs. 1-4), having occupancies 0.66 (2) and 0.34 (2) in (I),
0.81 (3) and 0.19 (3) in (II), 0.73 (2) and 0.27 (2) in (III) and 0.80 (2) and 0.20 (2) in (IV). Similarly, compounds (VIII) and (IX) are isomorphous, and in both of them the anion exhibits disorder, with occupancies of $0.660(15)$ and $0.340(15)$ in (VIII), and 0.906 (9) and 0.094 (9) in (IX) (Figs. 8 and 9).

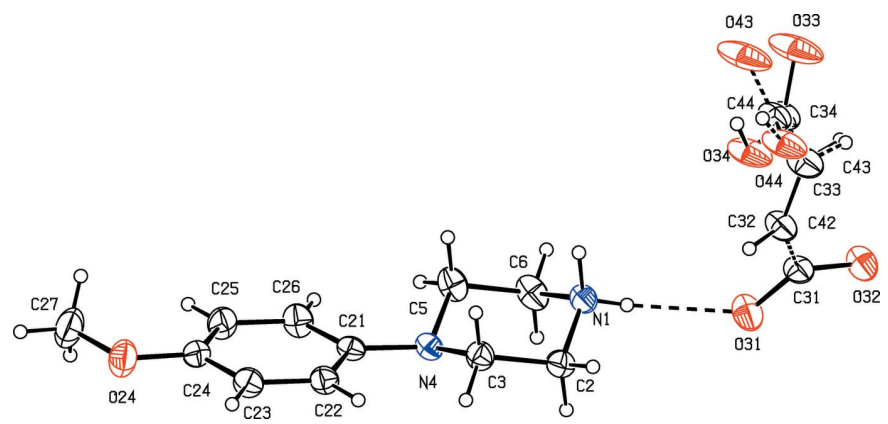

Figure 9

The independent components of compound (IX), showing the atomlabelling scheme, the disorder of anion, and the hydrogen bonds within the selected asymmetric unit. The major disorder component is drawn using full lines and the minor disorder component is drawn using dashed lines. Displacement ellipsoids are drawn at the $30 \%$ probability level.

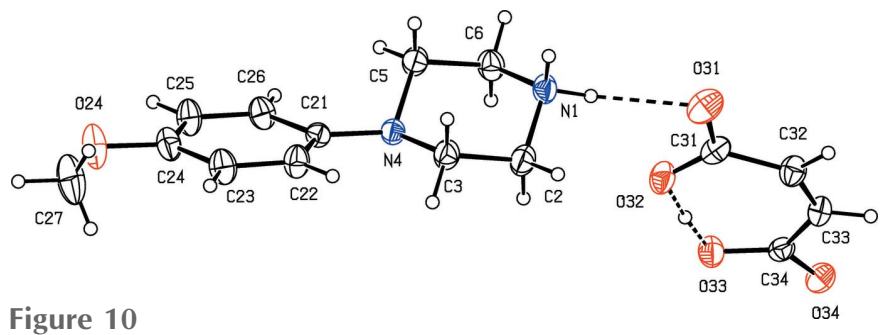

Figure 10

The independent components of compound (X), showing the atomlabelling scheme and the hydrogen bonds within the selected asymmetric unit. Displacement ellipsoids are drawn at the $30 \%$ probability level.

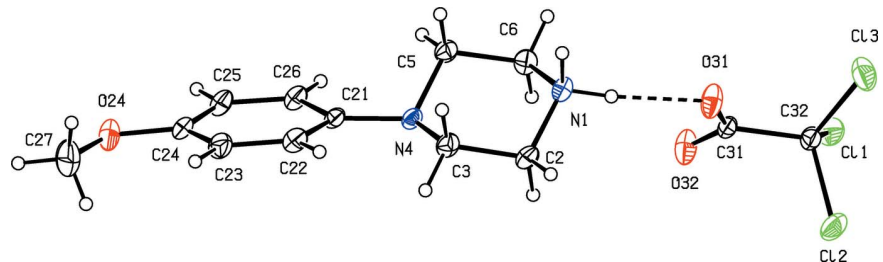

Figure 11

The independent components of compound (XI), showing the atomlabelling scheme and the hydrogen bond within the selected asymmetric unit. Displacement ellipsoids are drawn at the $30 \%$ probability level.

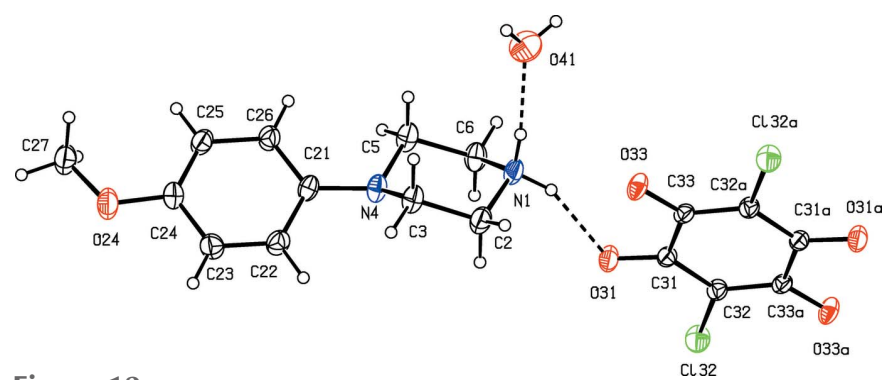

Figure 12

The independent components of compound (XII), showing the atomlabelling scheme and the hydrogen bonds within the selected asymmetric unit. Displacement ellipsoids are drawn at the $30 \%$ probability level, and the atoms marked with the suffix ' $\mathrm{a}$ ' are at the symmetry position $(1-x$, $1-y,-z)$ 
While compounds (I)-(IV) are isostructural, compounds (VIII) and (IX) are not, because of both the different configurations of their anions and the different degrees of disorder. Examples have been previously reported of compounds that are isomorphous but not strictly isostructural in terms of their intermolecular interactions (Acosta et al., 2009).

In the anion of compound (VII), the carboxyl group is unionized, with $\mathrm{C}-\mathrm{O}$ distances of 1.220 (3) and 1.309 (3) $\AA$ and it is the phenolic $\mathrm{H}$ atom which has been lost (Fig. 7). The C32-O33 distance, 1.280 (3) $\AA$, is closer to that normally found in ketones than to that typical of phenols or phenolates (Allen et al., 1987): in addition, the C31-C32 and C32-C33 distances, 1.437 (4) and 1.430 (4) $\AA$, respectively, are significantly larger than the other $\mathrm{C}-\mathrm{C}$ distances in this ring, which lie in the rather narrow range 1.370 (3)-1.385 (4) $\AA$, but the $\mathrm{C}-\mathrm{N}$ and $\mathrm{N}-\mathrm{O}$ distances are all typical of their types (Allen et al., 1987). These observations indicate that the negative charge in this anion is delocalized over the five atoms C31, C33, C34, C35 and C36, but without any significant delocalization onto the nitro groups, as has been observed in trinitrophenolate (picrate) anions (Kavitha et al., 2006; Sagar et al., 2017; Shaibah et al., 2017a,b).

The anion of compound (X) contains an almost linear and very short (Emsley, 1980; Herschlag \& Pinney, 2018) $\mathrm{O} \cdots \mathrm{H} \cdots \mathrm{O}$ hydrogen bond, in which the $\mathrm{H}$ atom is almost, but not exactly, centred between the two $\mathrm{O}$ atoms (Table 1). In the centrosymmetric anion of compound (XII) (Fig. 12), the two
(A)

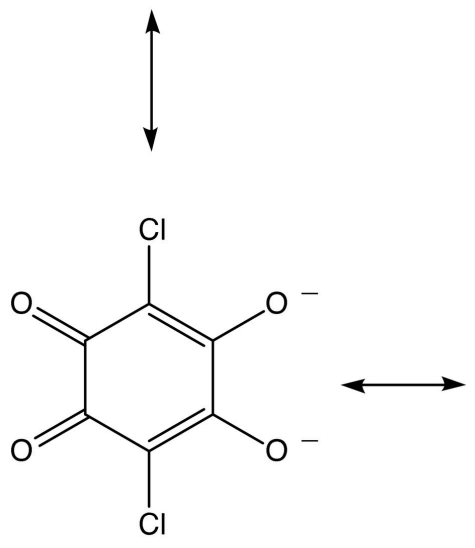

(C)<smiles>CCCCCO</smiles><smiles>COC1=C(Cl)C(=O)C(OC)=C(Cl)C1=O</smiles>

(B)<smiles>CC1([Tl])CCCCC1</smiles><smiles>O=C1C(=O)C(Cl)=C(O)C(O)=C1Cl</smiles>

(D)

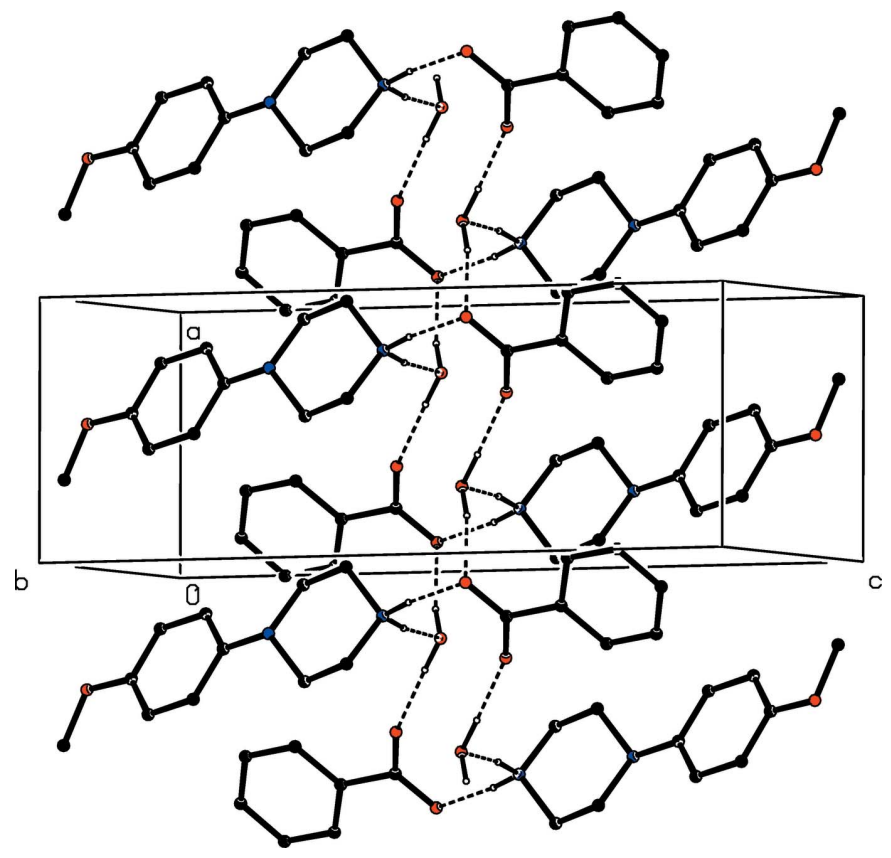

Figure 14

Part of the crystal structure of compound (I) showing the formation of a chain of rings parallel to the [100] direction. Hydrogen bonds are drawn as dashed lines and, for the sake of clarity, the minor disorder component and the $\mathrm{H}$ atoms bonded to $\mathrm{C}$ atoms have been omitted.

independent $\mathrm{C}-\mathrm{O}$ distances are identical within experimental uncertainty, $1.244(2)$ and $1.246(2) \AA$, as are the distances C31-C32 and C32-C33, 1.398 (3) and 1.392 (2) A. However, the remaining $\mathrm{C}-\mathrm{C}$ distance in this ring, 1.539 (3) $\AA$ is typical of a single C-C bond (Allen et al., 1987). These observations indicates the delocalization of a negative charge across each of the $\mathrm{O}-\mathrm{C}-\mathrm{C}-\mathrm{C}-\mathrm{O}$ units, and that these two units are effectively isolated from each other electronically. Despite the apparent simplicity of this dianion, with its high intrinsic symmetry, it is not possible adequately to describe its electronic structure in a single diagrammatic form, and four forms $(A)-(D)$ (Fig. 13) are required.

\section{Supramolecular features}

In each of the four isomorphous salts (I)-(IV), the ions are linked by a combination of $\mathrm{N}-\mathrm{H} \cdots \mathrm{O}$ and $\mathrm{O}-\mathrm{H} \cdots \mathrm{O}$ hydrogen bonds (Table 1) to form a chain of edge-fused centrosymmetric rings running parallel to the [100] direction, in which $R_{6}^{4}(12)$ (Etter, 1990; Etter et al., 1990; Bernstein et al., 1995) rings centred at $\left(n, \frac{1}{2}, \frac{1}{2}\right)$ alternate with $R_{6}^{6}(16)$ rings centred at $\left(n+\frac{1}{2}, \frac{1}{2}, \frac{1}{2}\right)$, where $n$ represents an integer in each case (Fig. 14). In each of these four salts, a combination of $\mathrm{C}-$ $\mathrm{H} \cdots \mathrm{O}$ and $\mathrm{C}-\mathrm{H} \cdots \pi$ (arene) hydrogen bonds links the [100] chain into complex sheets lying parallel to (001).

There is an intermolecular $\mathrm{O}-\mathrm{H} \cdots \mathrm{O}$ hydrogen bond in the anion of the unsolvated salt (V). The two anions in the selected asymmetric unit (Fig. 5) are linked by an asymmetric three-centre $\mathrm{N}-\mathrm{H} \cdots(\mathrm{O})_{2}$ hydrogen bond, and the resulting ion pairs, which are related by $2_{1}$ screw axis along $\left(\frac{1}{2}, y, \frac{1}{4}\right)$, are 
Table 1

Hydrogen-bond parameters and short intermolecular contacts $\left(\AA{ }^{\circ}{ }^{\circ}\right)$.

$C g 1$ and $C g 2$ are the centroids of the $\mathrm{C} 31-\mathrm{C} 36$ and $\mathrm{C} 21-\mathrm{C} 26$ rings, respectively.

\begin{tabular}{|c|c|c|c|c|c|}
\hline Compound & $D-\mathrm{H} \cdots A$ & $D-\mathrm{H}$ & $\mathrm{H} \cdots A$ & $D \cdots A$ & $D-\mathrm{H} \cdots A$ \\
\hline & $\mathrm{N} 1-\mathrm{H} 12 \cdots \mathrm{O} 41$ & $0.97(2)$ & $1.85(2)$ & $2.808(3)$ & $169.7(18)$ \\
\hline & $\mathrm{O} 41-\mathrm{H} 41 \cdots \mathrm{O} 32^{\mathrm{i}}$ & $0.88(3)$ & $1.75(3)$ & $2.631(3)$ & $177(3)$ \\
\hline & $\mathrm{C} 2-\mathrm{H} 2 B \cdots \mathrm{O} 31^{\mathrm{iii}}$ & 0.97 & 2.54 & $3.485(3)$ & 165 \\
\hline & $\mathrm{C} 22-\mathrm{H} 22 \cdots C g 1^{\mathrm{ii}}$ & 0.93 & 2.85 & $3.603(3)$ & 139 \\
\hline & $\mathrm{C} 26-\mathrm{H} 26 \cdots C g 1^{\text {iv }}$ & 0.93 & 2.90 & $3.62(2)$ & 135 \\
\hline \multirow{6}{*}{ (II) } & $\mathrm{N} 1-\mathrm{H} 12 \cdots \mathrm{O} 41$ & $0.86(3)$ & $1.96(3)$ & $2.818(4)$ & $170(3)$ \\
\hline & $\mathrm{O} 41-\mathrm{H} 41 \cdots \mathrm{O} 32^{\mathrm{i}}$ & $0.86(4)$ & $1.75(4)$ & $2.627(4)$ & $174(4)$ \\
\hline & $\mathrm{O} 41-\mathrm{H} 42 \cdots \mathrm{O} 31^{\mathrm{ii}}$ & $0.91(4)$ & $1.88(4)$ & $2.768(3)$ & $163(3)$ \\
\hline & $\mathrm{C} 2-\mathrm{H} 2 B \cdots \mathrm{O} 31^{\mathrm{iii}}$ & 0.97 & 2.58 & $3.529(4)$ & 166 \\
\hline & $\mathrm{C} 6-\mathrm{H} 6 B \cdots \mathrm{O} 41^{\mathrm{i}}$ & 0.97 & 2.57 & $3.386(4)$ & 142 \\
\hline & $\mathrm{C} 26-\mathrm{H} 26 \cdots C g 1^{\mathrm{iv}}$ & 0.93 & 2.81 & $3.56(2)$ & 138 \\
\hline \multirow{5}{*}{ (III) } & $\mathrm{O} 41-\mathrm{H} 42 \cdots \mathrm{O} 31^{\mathrm{ii}}$ & $0.94(4)$ & $1.84(4)$ & $2.775(4)$ & $170(4)$ \\
\hline & $\mathrm{C} 2-\mathrm{H} 2 B \cdots \mathrm{O} 31^{\mathrm{iii}}$ & 0.97 & 2.52 & 3.467 (4) & 165 \\
\hline & $\mathrm{C} 6-\mathrm{H} 6 B \cdots \mathrm{O} 41^{\mathrm{i}}$ & 0.97 & 2.60 & $3.408(4)$ & 141 \\
\hline & $\mathrm{C} 22-\mathrm{H} 22 \cdots C g 1^{\mathrm{iv}}$ & 0.93 & 2.89 & $3.631(13)$ & 137 \\
\hline & $\mathrm{C} 26-\mathrm{H} 26 \cdots C g 1^{\mathrm{iv}}$ & 0.93 & 2.81 & $3.58(2)$ & 141 \\
\hline \multirow[t]{7}{*}{ (IV) } & $\mathrm{N} 1-\mathrm{H} 11 \cdots \mathrm{O} 31$ & $0.78(4)$ & $2.03(4)$ & $2.805(5)$ & $174(5)$ \\
\hline & $\mathrm{N} 1-\mathrm{H} 12 \cdots \mathrm{O} 41$ & $0.95(5)$ & $1.86(5)$ & $2.802(5)$ & $172(4)$ \\
\hline & $\mathrm{O} 41-\mathrm{H} 41 \cdots \mathrm{O} 32^{\mathrm{i}}$ & $0.79(6)$ & $1.84(6)$ & $2.623(6)$ & $170(6)$ \\
\hline & $\mathrm{O} 41-\mathrm{H} 42 \cdots \mathrm{O} 31^{\mathrm{ii}}$ & $0.79(7)$ & $2.00(7)$ & $2.772(5)$ & $169(6)$ \\
\hline & $\mathrm{C} 2-\mathrm{H} 2 B \cdots \mathrm{O} 31^{\mathrm{iii}}$ & 0.97 & 2.52 & $3.471(5)$ & 166 \\
\hline & $\mathrm{C} 22-\mathrm{H} 22 \cdots C g 1^{\mathrm{ii}}$ & 0.93 & 2.52 & $3.471(5)$ & 166 \\
\hline & $\mathrm{C} 26-\mathrm{H} 26 \cdots C g 1^{\text {iv }}$ & 0.93 & 2.84 & $3.58(2)$ & 137 \\
\hline \multirow{4}{*}{ (VI) } & $\mathrm{N} 1-\mathrm{H} 12 \cdots \mathrm{O} 32^{\mathrm{ix}}$ & $0.94(2)$ & $1.82(2)$ & $2.749(2)$ & $168.3(17)$ \\
\hline & $\mathrm{C} 2-\mathrm{H} 2 B \cdots \mathrm{N} 31^{\mathrm{iv}}$ & 0.97 & 2.56 & $3.518(2)$ & 169 \\
\hline & $\mathrm{C} 36-\mathrm{H} 36 \cdots \mathrm{O} 24^{x}$ & 0.93 & 2.51 & $3.432(2)$ & 172 \\
\hline & $\mathrm{C} 3-\mathrm{H} 3 A \cdots C g 1^{\mathrm{xi}}$ & 0.97 & 2.97 & $3.775(2)$ & 156 \\
\hline \multirow[t]{7}{*}{ (VII) } & $\mathrm{O} 32-\mathrm{H} 32 \cdots \mathrm{O} 33$ & $1.04(4)$ & $1.47(4)$ & $2.472(3)$ & $158(3)$ \\
\hline & $\mathrm{N} 1-\mathrm{H} 11 \cdots \mathrm{O} 33$ & $0.93(3)$ & $1.98(3)$ & $2.020(3)$ & $150(3)$ \\
\hline & $\mathrm{N} 1-\mathrm{H} 11 \cdots \mathrm{O} 34$ & $0.93(3)$ & $2.27(3)$ & $2.910(3)$ & $126(2)$ \\
\hline & $\mathrm{N} 1-\mathrm{H} 12 \cdots \mathrm{O} 31^{\mathrm{i}}$ & $0.93(3)$ & $2.04(3)$ & $2.931(3)$ & $160(3)$ \\
\hline & $\mathrm{N} 1-\mathrm{H} 12 \cdots \mathrm{O} 32^{\mathrm{i}}$ & $0.93(3)$ & $2.58(3)$ & $3.250(3)$ & $129(2)$ \\
\hline & $\mathrm{C} 34-\mathrm{H} 34 \cdots \mathrm{O} 36^{\mathrm{xii}}$ & 0.93 & 2.53 & 3.449 (3) & 171 \\
\hline & $\mathrm{C} 5-\mathrm{H} 5 B \cdots C g 2^{\mathrm{xiii}}$ & 0.97 & 2.84 & $3.639(3)$ & 140 \\
\hline \multirow[t]{7}{*}{ (VIII) } & $\mathrm{N} 1-\mathrm{H} 11 \cdots \mathrm{O} 31$ & $0.86(3)$ & $1.90(3)$ & $2.750(15)$ & $167(4)$ \\
\hline & $\mathrm{N} 1-\mathrm{H} 12 \cdots \mathrm{O} 32^{\mathrm{xiv}}$ & $0.98(3)$ & $1.77(4)$ & 2.741 (19) & $171(3)$ \\
\hline & $\mathrm{O} 34-\mathrm{H} 34 \cdots \mathrm{O} 31^{\mathrm{xv}}$ & 0.82 & 1.79 & $2.60(2)$ & 168 \\
\hline & $\mathrm{N} 1-\mathrm{H} 11 \cdots \mathrm{O} 41$ & $0.86(3)$ & $2.18(4)$ & $3.03(3)$ & $165(4)$ \\
\hline & $\mathrm{N} 1-\mathrm{H} 12 \cdots \mathrm{O} 42^{\mathrm{xiv}}$ & $0.98(3)$ & $1.82(5)$ & $2.77(4)$ & $163(3)$ \\
\hline & $\mathrm{O} 44-\mathrm{H} 44 \cdots \mathrm{O} 41^{\mathrm{xv}}$ & 0.82 & 1.56 & $2.35(2)$ & 161 \\
\hline & $\mathrm{C} 3-\mathrm{H} 3 A \cdots C g 2^{\mathrm{xvi}}$ & 0.97 & 2.76 & $3.652(3)$ & 154 \\
\hline \multirow[t]{4}{*}{ (IX) } & $\mathrm{N} 1-\mathrm{H} 11 \cdots \mathrm{O} 31$ & $0.81(4)$ & $2.18(3)$ & $2.940(4)$ & $155(3)$ \\
\hline & $\mathrm{N} 1-\mathrm{H} 12 \cdots \mathrm{O} 32^{\mathrm{xiv}}$ & $0.96(4)$ & $1.77(4)$ & $2.714(4)$ & $169(3)$ \\
\hline & $\mathrm{O} 34-\mathrm{H} 34 \cdots \mathrm{O} 31^{\mathrm{xv}}$ & 0.82 & 1.71 & $2.522(5)$ & 170 \\
\hline & $\mathrm{O} 43-\mathrm{H} 34 \cdots \mathrm{O} 31^{\mathrm{xv}}$ & 0.82 & 1.62 & $2.44(2)$ & 175 \\
\hline
\end{tabular}


Table 1 (continued)

\begin{tabular}{|c|c|c|c|c|c|}
\hline Compound & $D-\mathrm{H} \cdots A$ & $D-\mathrm{H}$ & $\mathrm{H} \cdots A$ & $D \cdots A$ & $D-\mathrm{H} \cdots A$ \\
\hline & $\mathrm{C} 3-\mathrm{H} 3 A \cdots C g 2^{\mathrm{xvi}}$ & 0.97 & 2.76 & $3.650(3)$ & 153 \\
\hline \multirow[t]{4}{*}{$(X)$} & $\mathrm{O} 33-\mathrm{H} 33 A \cdots \mathrm{O} 32$ & $1.167(18)$ & $1.247(18)$ & $2.4121(16)$ & $175(2)$ \\
\hline & $\mathrm{N} 1-\mathrm{H} 11 \cdots \mathrm{O} 32$ & $0.915(17)$ & $2.296(17)$ & $3.0798(18)$ & $143.5(14)$ \\
\hline & $\mathrm{N} 1-\mathrm{H} 12 \cdots \mathrm{O} 34^{\mathrm{xvii}}$ & $0.919(18)$ & $1.881(18)$ & $2.7563(17)$ & $158.5(17)$ \\
\hline & $\mathrm{C} 2-\mathrm{H} 2 A \cdots \mathrm{O} 34^{\mathrm{ii}}$ & 0.97 & 2.56 & $3.363(2)$ & 140 \\
\hline \multirow[t]{5}{*}{ (XII) } & $\mathrm{N} 1-\mathrm{H} 11 \cdots \mathrm{O} 31$ & $0.89(3)$ & $1.96(3)$ & $2.802(3)$ & $157(2)$ \\
\hline & $\mathrm{N} 1-\mathrm{H} 11 \cdots \mathrm{O} 33$ & $0.89(3)$ & $2.29(2)$ & $2.838(3)$ & $119(2)$ \\
\hline & $\mathrm{N} 1-\mathrm{H} 12 \cdots \mathrm{O} 41$ & $0.90(2)$ & $1.92(2)$ & $2.798(3)$ & $168(3)$ \\
\hline & $\mathrm{O} 41-\mathrm{H} 41 \cdots \mathrm{O} 33^{\mathrm{i}}$ & $0.84(4)$ & $1.92(4)$ & $2.738(3)$ & $166(3)$ \\
\hline & $\mathrm{O} 41-\mathrm{H} 42 \cdots \mathrm{O} 24^{\mathrm{xix}}$ & $0.82(3)$ & $2.49(3)$ & $3.269(3)$ & $160(3)$ \\
\hline
\end{tabular}

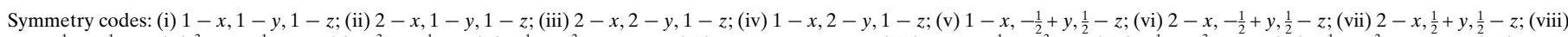

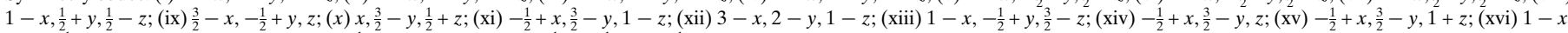
$1-y, \frac{1}{2}+z ;(\mathrm{xvii})-1+x, y, z$; (xviii) $-\frac{1}{2}+x, 1-y, z ;(\mathrm{xix})-\frac{1}{2}+x, \frac{1}{2}-y,-\frac{1}{2}+z$.

linked by a two-centre $\mathrm{N}-\mathrm{H}$... O hydrogen bond to form chain of rings running parallel to the [010] direction (Fig. 15). Chains of this type are weakly linked into sheets lying parallel to (001) by a combination of $\mathrm{C}-\mathrm{H} \cdots \mathrm{O}$ and $\mathrm{C}-\mathrm{H} \cdots \pi$ (arene) hydrogen bonds.

The component ions in compound (VI) (Fig. 6) are linked by a two-centre $\mathrm{N}-\mathrm{H} \cdots \mathrm{O}$ hydrogen bond and the resulting

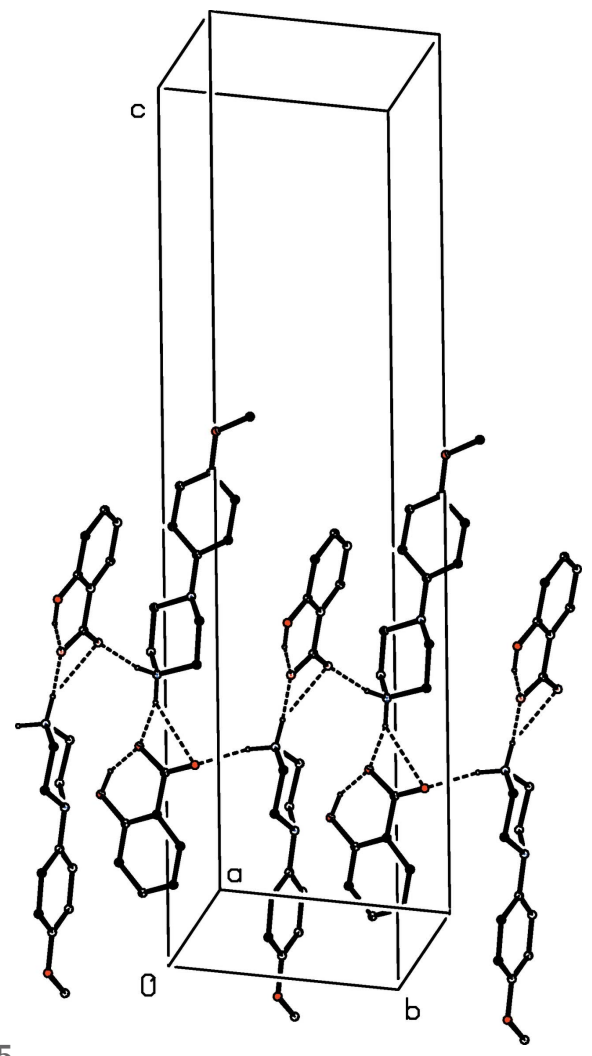

Figure 15

Part of the crystal structure of compound (V) showing the formation of a chain of rings parallel to the [010] direction. Hydrogen bonds are drawn as dashed lines and, for the sake of clarity, the $\mathrm{H}$ atoms bonded to $\mathrm{C}$ atoms have been omitted. ion pairs are further linked by a combination of $\mathrm{N}-\mathrm{H} \cdots \mathrm{O}$, $\mathrm{C}-\mathrm{H} \cdots \mathrm{O}$ and $\mathrm{C}-\mathrm{H} \cdots \mathrm{N}$ hydrogen bonds to form a threedimensional framework structure, whose formation can readily be analysed in terms of three simple sub-structures (Ferguson et al., 1998a,b; Gregson et al., 2000). Ion pairs which are related by the $b$-glide plane at $x=\frac{3}{4}$ are linked by a second $\mathrm{N}-\mathrm{H} \cdots \mathrm{O}$ hydrogen bond to form a $C_{2}^{2}(6)$ chain running

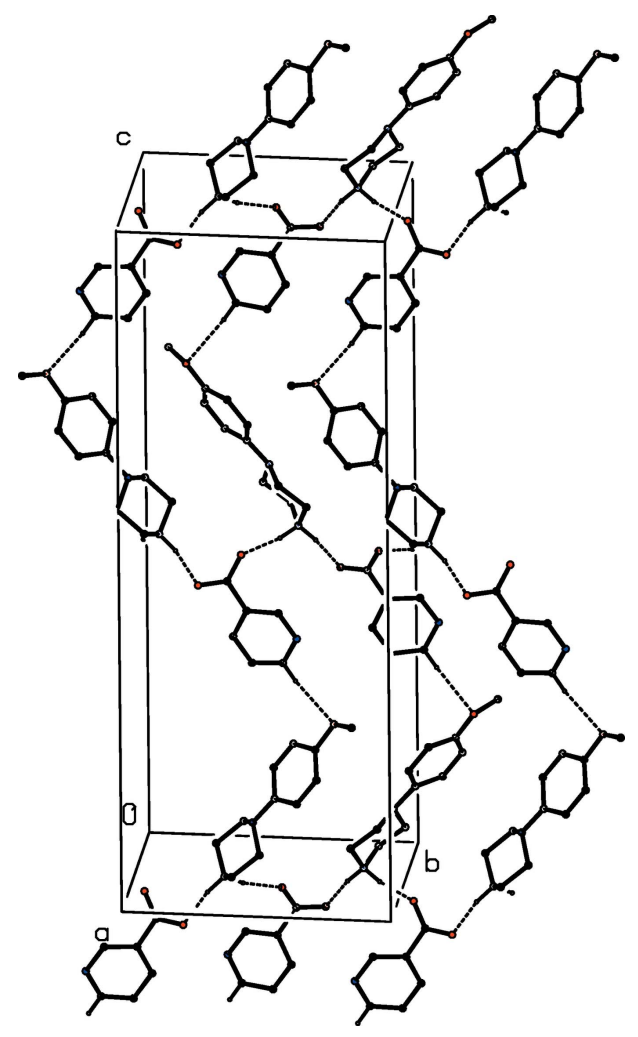

Figure 16

Part of the crystal structure of compound (VI) showing the formation of a sheet of $R_{6}^{6}(40)$ rings lying parallel to (100). Hydrogen bonds are drawn as dashed lines and, for the sake of clarity, the $\mathrm{H}$ atoms not involved in the motifs shown have been omitted. 
parallel to the [010] direction, and in the second sub-structure, ion pairs which are related by the $c$-glide plane at $y=\frac{3}{4}$ are linked by a $\mathrm{C}-\mathrm{H} \cdots \mathrm{O}$ hydrogen bond (Table 1 ) to form a $C_{2}^{2}(17)$ chain running parallel to the [001] direction. The combination of these two simple chain motifs generates a sheet of $R_{6}^{6}(40)$ rings lying parallel to (100) in the domain $\frac{1}{2}<x<1.0$ (Fig. 16). A second sheet of this type, related to the first by inversion lies in the domain $0<x<\frac{1}{2}$, and adjacent sheets are linked by the third sub-structure in which inversionrelated ion pairs are linked by $\mathrm{C}-\mathrm{H} \cdots \mathrm{N}$ hydrogen bonds into a centrosymmetric $R_{4}^{4}(18)$ ring (Fig. 17): the action of this interaction is to link all of the (100) sheets into a continuous three-dimensional array.

There is an intermolecular $\mathrm{O}-\mathrm{H} \cdots \mathrm{O}$ hydrogen bond in the anion of compound (VII) (Fig. 7), but the carboxyl $\mathrm{H}$ atom plays no part in the supramolecular assembly. The ions are linked by a combination of $\mathrm{N}-\mathrm{H} \cdots \mathrm{O}$ and $\mathrm{C}-\mathrm{H} \cdots \mathrm{O}$ hydrogen bonds to form a chain of centrosymmetric rings

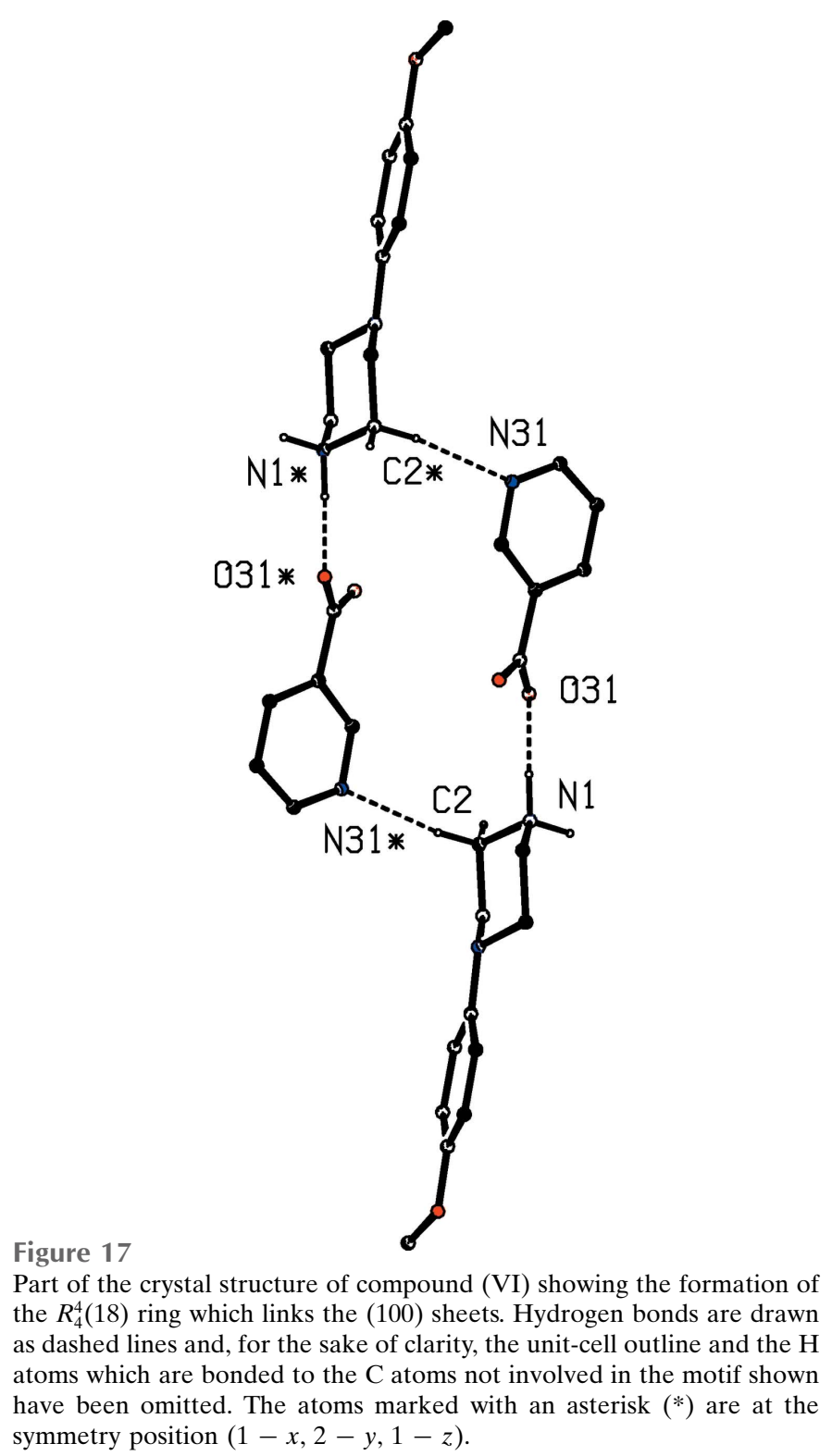

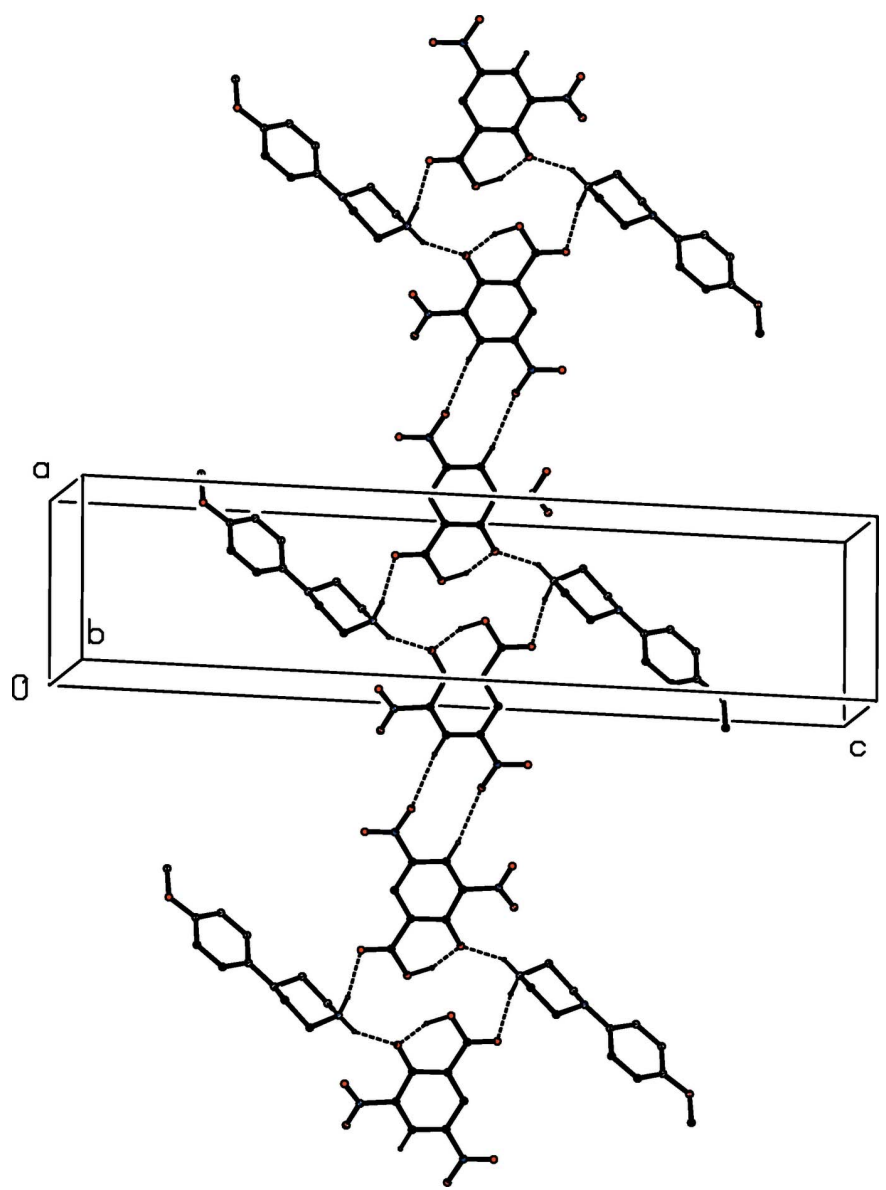

Figure 18

Part of the crystal structure of compound (VII) showing the formation of a chain of $R_{2}^{2}(10)$ and $R_{6}^{4}(16)$ rings parallel to the [210] direction. Hydrogen bonds are drawn as dashed lines and, for the sake of clarity, the $\mathrm{H}$ atoms which are bonded to the $\mathrm{C}$ atoms not involved in the motif shown have been omitted.

running parallel to the [210] direction, in which $R_{2}^{2}(10)$ rings centred at $\left(2 n-\frac{1}{2}, n, \frac{1}{2}\right)$ alternate with $R_{6}^{4}(16)$ rings centred at $\left(2 n+\frac{1}{2}, n+\frac{1}{2}, \frac{1}{2}\right)$, where $n$ represents an integer in each case (Fig. 18). Two chains of this type, related to one another by the translational symmetry operations, pass through each unit cell, and a weak $\mathrm{C}-\mathrm{H} \cdots \pi$ (arene) hydrogen bond links the chains into a three-dimensional framework structure.

For the disordered structure of compound (VIII), the hydrogen bonds formed by the major and minor disorder components are very similar (Table 1) so that only the major disorder form need be considered in detail. Within the selected asymmetric unit (Fig. 8), the component ions are linked by a two-centre $\mathrm{N}-\mathrm{H} \cdots \mathrm{O}$ hydrogen bond: the ion pairs are linked by a combination of $\mathrm{N}-\mathrm{H} \cdots \mathrm{O}$ and $\mathrm{O}-$ $\mathrm{H}$. . O hydrogen bonds to form sheets, whose formation can readily be analysed in terms of two simple sub-structures. In the simpler of these, anions which are related by the $a$-glide plane at $y=\frac{3}{4}$ are linked by $\mathrm{O}-\mathrm{H} \cdots \mathrm{O}$ hydrogen bonds into $C(7)$ chains running parallel to the [10̄] direction (Fig. 19); in the second sub-structure, ion pairs which are related by the same glide plane are linked by $\mathrm{N}-\mathrm{H} \cdots \mathrm{O}$ hydrogen bonds to form a $C_{2}^{2}(6)$ chain running parallel to the [100] direction 


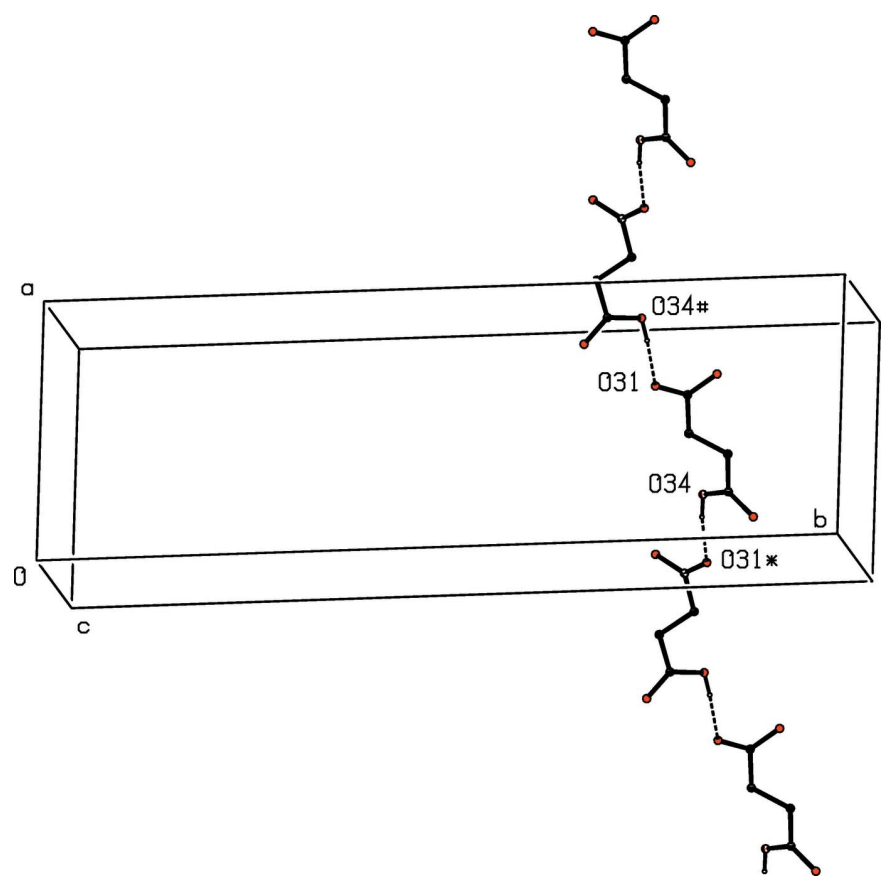

Figure 19

Part of the crystal structure of compound (VIII) showing the formation of a $C(7)$ chain of anions, parallel to [102] . Hydrogen bonds are drawn as dashed lines and, for the sake of clarity, the $\mathrm{H}$ atoms bonded to $\mathrm{C}$ atoms have been omitted. The atoms marked with an asterisk $(*)$ or a hash (\#) are at the symmetry positions $\left(\frac{1}{2}+x, \frac{3}{2}-y,-1+z\right)$ and $\left(-\frac{1}{2}+x, \frac{3}{2}-y\right.$, $1+z)$, respectively.

(Fig. 20). The combination of these two chain motifs generates a sheet lying parallel to (010), and a single $\mathrm{C}-\mathrm{H} \cdots \pi$ (arene) hydrogen bond links these sheets into a three-dimensional framework structure. The supramolecular aggregation in the isomorphous compound (IX) is similar to that in (VIII). As noted in Section 2 above, the anion in compound (X) contains

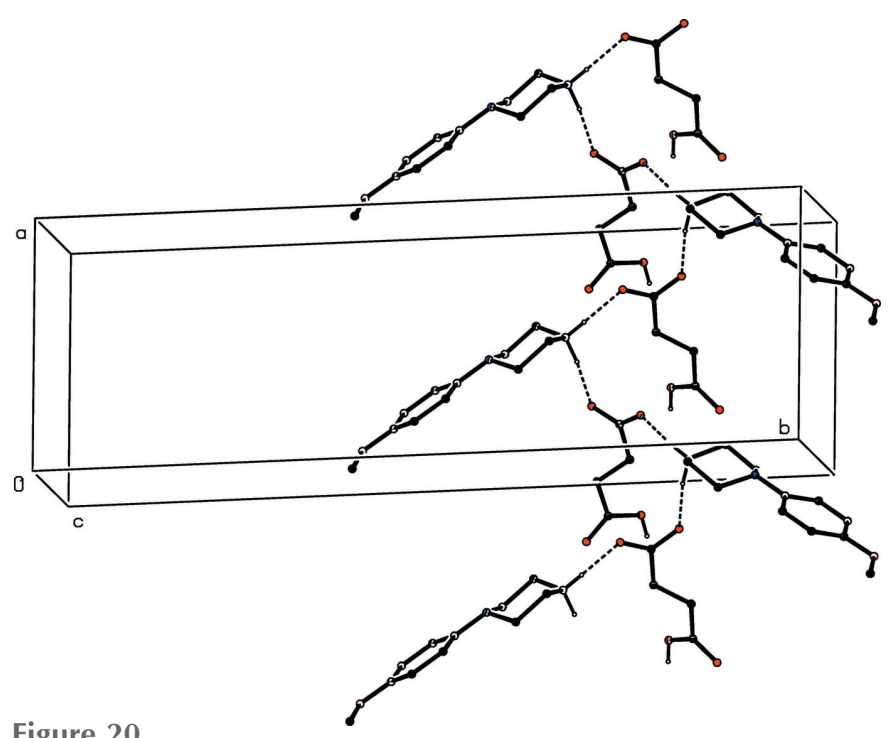

Figure 20

Part of the crystal structure of compound (VIII) showing the formation of a $C_{2}^{2}(6)$ chain parallel to [100]. Hydrogen bonds are drawn as dashed lines and, for the sake of clarity, the $\mathrm{H}$ atoms bonded to $\mathrm{C}$ atoms have been omitted.

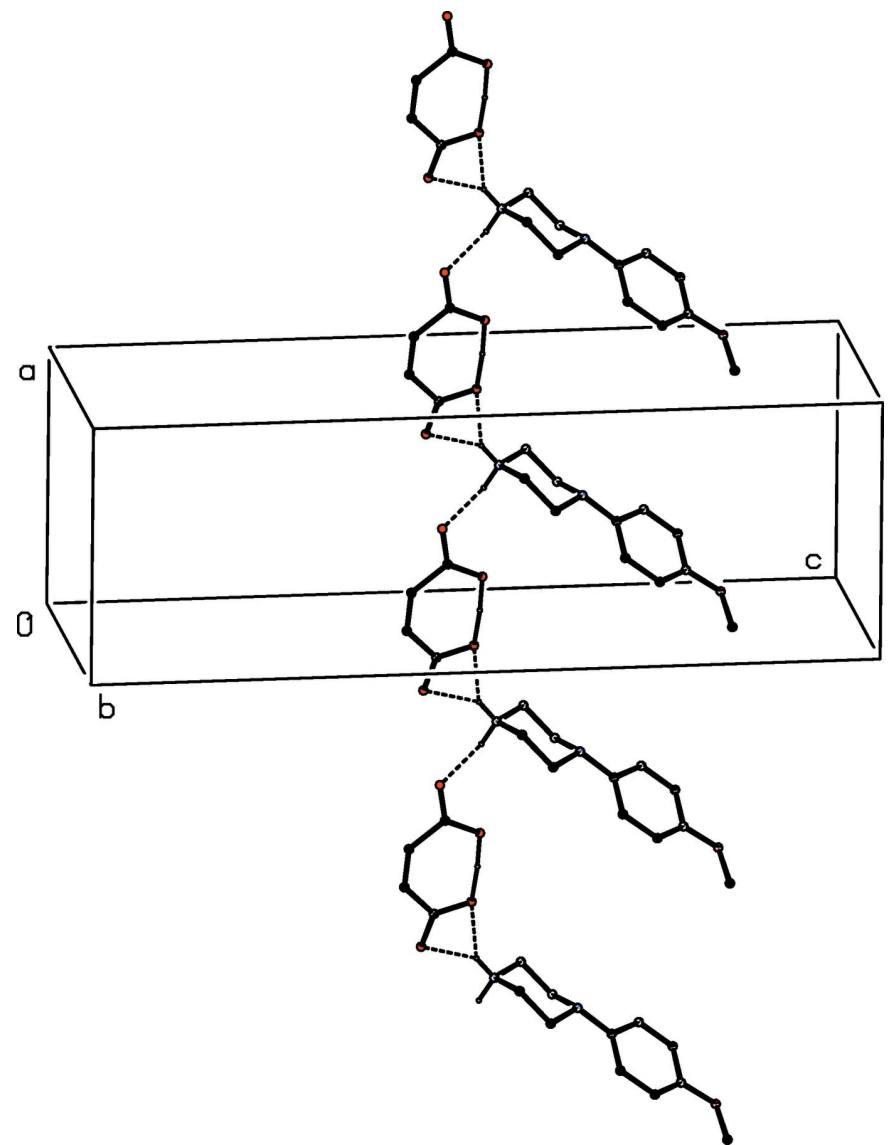

Figure 21

Part of the crystal structure of compound (X) showing the formation of a chain of rings parallel to [100]. Hydrogen bonds are drawn as dashed lines and, for the sake of clarity, the $\mathrm{H}$ atoms bonded to $\mathrm{C}$ atoms have been omitted.

a very short and nearly symmetrical $\mathrm{O} \cdots \mathrm{H} \cdots \mathrm{O}$ hydrogen bond. Within the selected asymmetric unit, the component ions are linked by the three-centre $\mathrm{N}-\mathrm{H} \cdots(\mathrm{O})_{2}$ hydrogen bond and ion pairs which are related by translation are linked by a two-centre $\mathrm{N}-\mathrm{H} \cdots \mathrm{O}$ hydrogen bond to form a $C(9) C(9)\left[R_{1}^{2}(4)\right]$ chain of rings running parallel to the [100] direction (Fig. 21). The $\mathrm{C}-\mathrm{H} \cdots \mathrm{O}$ contact is at the margin of significance (Wood et al., 2009), but it involves chains related by inversion.

The supramolecular assembly of compound (XI) is extremely simple: two $\mathrm{N}-\mathrm{H}$. . O hydrogen bonds link the ions into a $C_{2}^{2}(6)$ chain running parallel to the [100] direction (Fig. 22). In compound (XII), a combination of $\mathrm{N}-\mathrm{H} \cdots \mathrm{O}$ and $\mathrm{O}-$ $\mathrm{H}$. . O hydrogen bonds links all three components into a chain of $R_{6}^{6}(18)$ rings running parallel to the [001] direction (Fig. 23), while a second $\mathrm{O}-\mathrm{H}$. . O hydrogen bond links a combination of cations and water molecules into a simple $C_{2}^{2}(12)$ chain running parallel to the [101] direction (Fig. 24) and the combination of these two chain motifs generates a complex sheet lying parallel to (010).

Overall, therefore, the hydrogen-bonded assembly is onedimensional in each of compounds (X) and (XI), twodimensional in compounds (I)-(V) and (XII), and threedimensional in compounds (VI)-(IX). Sub-structures in the 


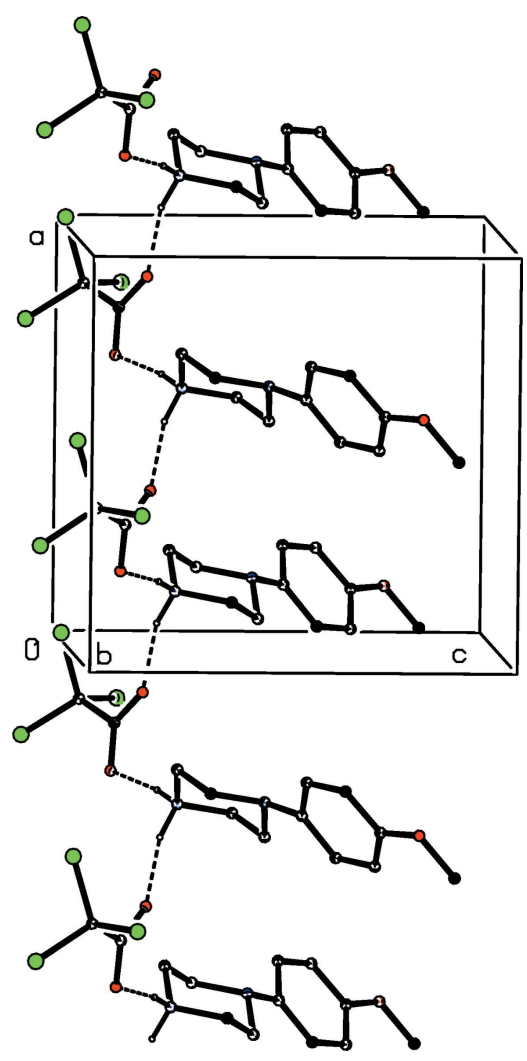

Figure 22

Part of the crystal structure of compound (XI) showing the formation of a $C_{2}^{2}(6)$ chain parallel to [100]. Hydrogen bonds are drawn as dashed lines and, for the sake of clarity, the $\mathrm{H}$ atoms bonded to $\mathrm{C}$ atoms have been omitted.

form of chains of rings can be identified in compounds (I)(IV) and in (VII), although (I)-(IV) are all monohydrates, while (VII) is solvent free: within the chain of rings formed by (I)-(IV) it is possible to identify a $C_{2}^{2}(6)$ motif formed by water molecules and anions only (Fig. 14), and a $C_{2}^{2}(6)$ motif built from alternating cations and anions can, in fact, be identified in each of compounds (V), (VI), (VIII), (IX) and (XI) (Figs. $15,16,20,22)$. By contrast, a $C_{2}^{2}(12)$ motif, built from water molecules and cations can be identified in the structure of compound (XII) (Fig. 24), but sub-structural motifs in the form of simple chains are uncommon in this series (Fig. 19).

\section{Database survey}

Compounds (I)-(IV), reported here, are isomorphous across the series of anions $4-\mathrm{XC}_{6} \mathrm{H}_{4} \mathrm{COO}^{-}$, where $X=\mathrm{H}, \mathrm{F}, \mathrm{Cl}$ or $\mathrm{Br}$, despite the rather disparate sizes of the substituents $X$. A similar, but more extreme, series of isomorphous salts was found in the substituted anilinium 5-nitro(hydrogenphthalate) salts $\left(4-\mathrm{XC}_{6} \mathrm{H}_{4} \mathrm{NH}_{3}\right)^{+} \cdot\left(\mathrm{C}_{8} \mathrm{H}_{4} \mathrm{NO}_{6}\right)^{-}$, which are isomorphous for $X=\mathrm{H}, \mathrm{Cl}, \mathrm{Br}$ and I (Glidewell et al., 2005). The structures of a number of salts containing the chloranilate dianion have been reported (Ishida, 2004a,b,c,d; Sovago et al., 2016), and the geometric features previously observed in this anion are fully consistent with the geometry found here in (XII): the

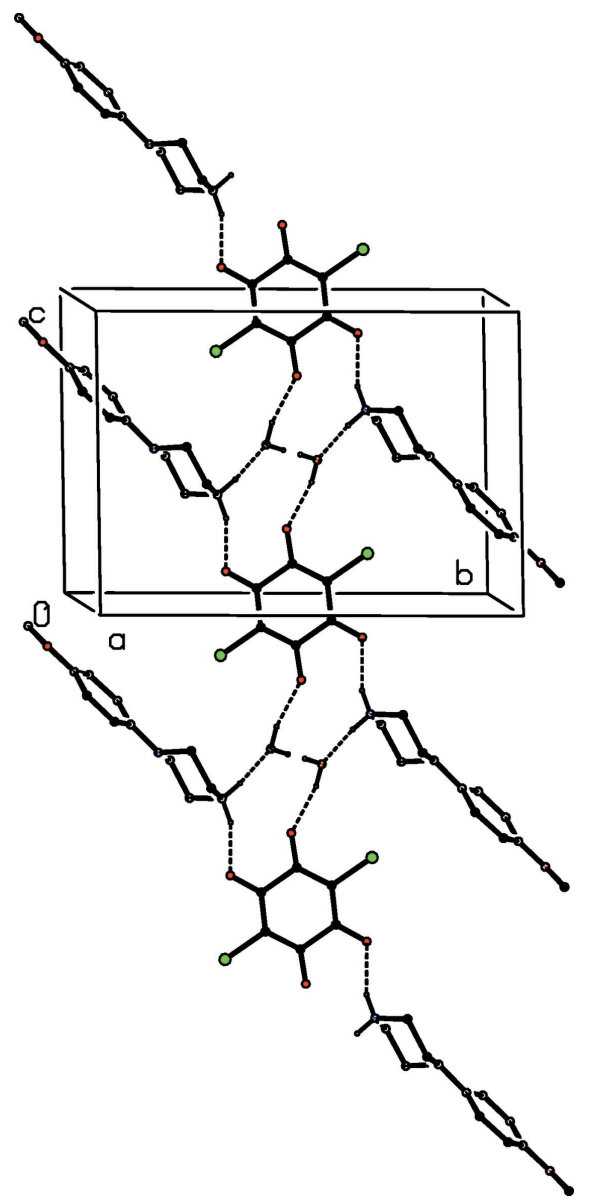

Figure 23

Part of the crystal structure of compound (XII) showing the formation of an $R_{6}^{6}(18)$ chain of rings parallel to [001]. Hydrogen bonds are drawn as dashed lines and, for the sake of clarity, the $\mathrm{H}$ atoms bonded to $\mathrm{C}$ atoms have been omitted.

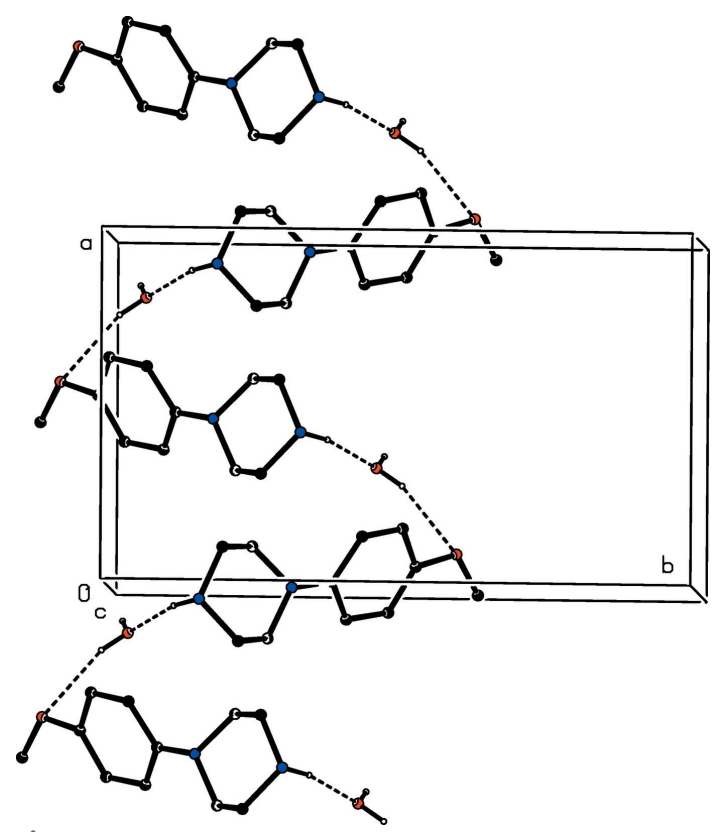

Figure 24

Part of the crystal structure of compound (XII) showing the formation of a $C_{2}^{2}(12)$ chain of cations and water molecules parallel to [101]. Hydrogen bonds are drawn as dashed lines and, for the sake of clarity, the $\mathrm{H}$ atoms bonded to $\mathrm{C}$ atoms have been omitted. 
nature of the electronic delocalization has been confirmed in several such salts using a combination of deformation density plots and net atomic charge calculations (Sovago et al., 2016).

The structures of very few salts containing the 4-(methoxyphenyl)piperazin-1-ium cations have been reported. In 4-(4-methoxyphenyl)piperazin-1-ium chloride, two N$\mathrm{H} \cdots \mathrm{Cl}$ hydrogen bonds link the ions into $C_{2}^{1}(4)$ chains (Zia-urRehman et al., 2009), and in the closely related 4-(4-nitrophenyl)piperazin-1-ium chloride monohydrate, a combination of $\mathrm{N}-\mathrm{H} \cdots \mathrm{O}, \mathrm{O}-\mathrm{H} \cdots \mathrm{Cl}$ and $\mathrm{N}-\mathrm{H} \cdots \mathrm{Cl}$ hydrogen bonds links the components into complex ribbons in which each anion accepts three hydrogen bonds $(\mathrm{Lu}, 2007)$. The structure of 4-(3-methoxyphenyl)piperazin-1-ium maleate has been reported (Verdonk et al., 1997), as have those of the picrate (Verdonk et al., 1997) and 6-chloro-5-isopropyl-2,4-dioxopyrimidin-1-ide (Al-Omary et al., 2014) salts of the 4-(2-methoxyphenyl)piperazin-1-ium cation. Finally we note, in addition to the 1-aroyl-4-(4-methoxyphenyl)piperazines referred to in Section 1 above (Kiran Kumar et al., 2019), the structure of 1-acetyl-(4-hydroxyphenyl)piperazine (Kavitha et al., 2013), which is an $\mathrm{N}$-acetylated derivative of 4-(4-hydroxyphenyl)piperazines, a metabolite of 4-(4-methoxyphenyl)piperazine.

\section{Synthesis and crystallization}

All reagents were obtained commercially and were used as received. For the synthesis of each of compounds (I)-(XII), equimolar quantities $(0.52 \mathrm{mmol}$ of each component) of $\mathrm{N}$-(4methoxyphenyl)piperazine and the appropriate acid were separately dissolved in methanol $(10 \mathrm{ml})$ and the two solutions were then mixed, stirred briefly, and then set aside to crystallize, giving the solid products (I)-(XII) after a few days. The products were all collected by filtration and then dried in air. Yields (I) $81 \%$, (II) $83 \%$, (III) $83 \%$, (IV) $81 \%$, (V) $83 \%$, (VI) $78 \%$, (VII) $80 \%$, (VIII) $82 \%$, (IX) $82 \%$, (X) $84 \%$, (XI) $79 \%$, (XII) $82 \%$ : melting ranges (I) $513-515 \mathrm{~K}$, (II) $405-407 \mathrm{~K}$, (III) 449-451 K, (IV) 447-449 K, (V) 471-473 K, (VI) 441-443 K, (VII) 475-477 K, (VIII) 439-441 K, (IX) 483-485 K, (X) 429431 K, (XI) 393-395 K, (XII) 575-577 K. Spectroscopic data (IR and ${ }^{1} \mathrm{H}$ NMR) are provided in the supporting information. Crystals of compounds (I), (II), and (VIII)-(XII) suitable for single-crystal X-ray diffraction analysis were selected directly from the prepared samples. Crystals of compounds (III)-(VII) suitable for single-crystal X-ray diffraction analysis were grown by slow evaporation, at ambient temperature and in the presence of air, of solutions in methanol-ethyl acetate (initial composition $1: 1, v / v)$. A number of other acids were used in similar co-crystallization experiments but they did not provide crystal suitable for single-crystal X-ray diffraction, thus: 2- and 3-fluorobenzoic acids [cf. compound (II)], 2- and 3-chlorobenzoic acids [cf. compound (III)], 2- and 3-bromobenzoic acids [cf. compound (IV)], 2- and 3-iodobenzoic acids, phthalic acid, 3-methylbenzoic acid [cf. compound (I)], 2,4-dichlorobenzoic acid, crotonic and adipic acids [cf. compounds (VIII)$(\mathrm{X})]$, and ascorbic, aspartic and glutamic acids.

\section{Refinement}

Crystal data, data collection and structure refinement details are summarized in Table 2. In each of the isomorphous compounds (I)-(IV), the 4-methoxyphenyl group exhibits disorder over two sets of atomic sites, and in each of (VIII) and (IX), the anion exhibits disorder involving two sets of atomic sites having unequal occupancies. In each case, the bonded distances and the 1,3 non-bonded distances in the minor disorder component were restrained to be the same as the equivalent distances in the major disorder component, subject to s.u. values of 0.01 and $0.02 \AA$, respectively, and the anisotropic displacement parameters for pairs of partialoccupancy atoms occupying essentially the same physical space were constrained to be equal: in addition, it was found necessary to constrain the minor component of the carboxyl group in (IX) to be planar. The ratio of observed-to-unique data was only $39 \%$ for compounds (II) and (III): this is probably a consequence of the ambient temperature data collection allied to the disorder: in both (VII) and (IX), the average $U_{3} / U_{1}$ ratio was $>4.0$ : this may be consequence of the disorder. Apart from those in the minor disorder components of (I)-(IV), (VIII) and (IX), all $\mathrm{H}$ atoms were located in difference maps. The $\mathrm{H}$ atoms bonded to $\mathrm{C}$ atoms were then treated as riding atoms in geometrically idealized positions with $\mathrm{C}-\mathrm{H}$ distances of $0.93 \AA$ (alkenyl and aromatic), $0.96 \AA$ $\left(\mathrm{CH}_{3}\right)$ or $0.97 \AA\left(\mathrm{CH}_{2}\right)$, and with $U_{\text {iso }}(\mathrm{H})=k U_{\text {eq }}(\mathrm{C})$, where $k=$ 1.5 for the methyl groups which were permitted to rotate but not to tilt, and 1.2 for all other $\mathrm{H}$ atoms bonded to $\mathrm{C}$ atoms: the $\mathrm{H}$ atoms bonded to $\mathrm{C}$ atoms in the minor disorder components were included on the same basis. The $\mathrm{H}$ atoms bonded to $\mathrm{O}$ atoms in the disordered components of (VIII) and (IX) were treated as riding atoms with $\mathrm{O}-\mathrm{H}=0.82 \AA$ and $U_{\text {iso }}(\mathrm{H})=1.5 U_{\text {eq }}(\mathrm{O})$, For the $\mathrm{H}$ atoms bonded to $\mathrm{N}$ atoms, and for the $\mathrm{H}$ atoms bonded to $\mathrm{O}$ atoms in $(\mathrm{I})-(\mathrm{V}),(\mathrm{VII}),(\mathrm{X})$ and (XII), the atomic coordinates were refined with $U_{\text {iso }}(\mathrm{H})=$ $1.2 U_{\text {eq }}(\mathrm{N})$ or $1.5 U_{\text {eq }}(\mathrm{O})$, leading to the $\mathrm{N}-\mathrm{H}$ and $\mathrm{O}-\mathrm{H}$ distances shown in Table 1 . The refined occupancies for the disorder components were 0.66 (2) and 0.34 (2) in (I), 0.81 (3) and 0.19 (3) in (II), 0.73 (2) and 0.27 (2) in (III), 0.80 (2) and $0.20(2)$ in (IV), $0.660(15)$ and $0.340(15)$ in (VIII), and 0.906 (9) and 0.094 (9) in (IX). For compound (XI), the correct orientation of the structure relative to the polar axis direction was established using the Flack $x$ parameter (Flack, 1983), $x=0.11$ (7). However, for compounds (V), (VIII) and (IX), where there is very little resonant scattering the values of the Flack $x$ parameter were indeterminate (Flack \& Bernardinelli, 2000), with values $-0.3(5),-0.6$ (7) and -0.3 (4), respectively: hence in these three cases, the correct orientation of the structure with respect to the polar axis direction cannot be established, although this has no chemical significance. The refinement of (XII) was treated as a non-merohedral twin, with twin matrix $(-1,0,0 / 0,-1,0 / 0.496,0,1)$ and with refined twin fractions 0.2467 (9) and 0.7533 (9).

\section{Acknowledgements}

HKK thanks University of Mysore for research facilities. 
Table 2

Experimental details.

$\begin{array}{llll}\text { (I) } & \text { (II) } & \text { (III) } & \text { (IV) }\end{array}$

Crystal data

Chemical formula

$M_{\mathrm{r}}$

Crystal system, space group

Temperature (K)

$a, b, c(\AA)$

$\alpha, \beta, \gamma\left({ }^{\circ}\right)$

$V\left(\AA^{3}\right)$

$Z$

Radiation type

$\mu\left(\mathrm{mm}^{-1}\right)$

Crystal size (mm)

Data collection

Diffractometer

Absorption correction

$T_{\min }, T_{\max }$

No. of measured, independent 5751, 3442, 1839 and observed $[I>2 \sigma(I)]$ reflections

$R_{\text {int }}$

$(\sin \theta / \lambda)_{\max }\left(\AA^{-1}\right)$

0.029

0.618

Oxford Diffraction Xcalibur with Sapphire CCD

Multi-scan (CrysAlis RED;

Oxford Diffraction, 2009)

Refinement

$R\left[F^{2}>2 \sigma\left(F^{2}\right)\right], w R\left(F^{2}\right), S$

No. of reflections

No. of parameters

No. of restraints

$\mathrm{H}$-atom treatment

$\Delta \rho_{\max }, \Delta \rho_{\min }\left(\mathrm{e} \AA^{-3}\right)$

\section{$0.054,0.134,1.02$}

3442

256

17

$\mathrm{H}$ atoms treated by a mixture of independent and constrained refinement

$\mathrm{C}_{11} \mathrm{H}_{17} \mathrm{~N}_{2} \mathrm{O}^{+} \cdot \mathrm{C}_{7} \mathrm{H}_{4} \mathrm{FO}_{2}{ }^{-} \cdot \mathrm{H}_{2} \mathrm{O}$
350.38
Triclinic, $P \overline{1}$
293

$\mathrm{C}_{11} \mathrm{H}_{17} \mathrm{~N}_{2} \mathrm{O}^{+} \cdot \mathrm{C}_{7} \mathrm{H}_{4} \mathrm{ClO}_{2}{ }^{-} \cdot \mathrm{H}_{2} \mathrm{O}$ 366.83

Triclinic, $P \overline{1}$

293

6.256 (1), 7.489 (1), 19.097 (2)

6.211 (1), 7.481 (1), 20.144 (4)

84.19 (1), 86.98 (2), 84.62 (2)

885.4 (2)

Mo $K \alpha$

0.10

$0.40 \times 0.24 \times 0.04$

84.90 (2), 87.48 (2), 85.19 (2)

928.4 (3)

Mo $K \alpha$

0.23

$0.20 \times 0.16 \times 0.02$

$\mathrm{C}_{11} \mathrm{H}_{17} \mathrm{~N}_{2} \mathrm{O}^{+} \cdot \mathrm{C}_{7} \mathrm{H}_{4} \mathrm{BrO}_{2}{ }^{-} \cdot \mathrm{H}_{2} \mathrm{O}$

411.28

Triclinic, $P \overline{1}$

293

6.2004 (8), 7.4957 (9),

$20.440(2)$

85.08 (1), 87.37 (1), 85.00 (1) 942.17 (19)

2

Mo $K \alpha$

2.21

$0.48 \times 0.44 \times 0.16$

Oxford Diffraction Xcalibur with Sapphire CCD

Multi-scan (CrysAlis RED;

Oxford Diffraction, 2009)

$0.973,0.996$

$5760,3477,1355$

Oxford Diffraction Xcalibur with Sapphire CCD

Multi-scan (CrysAlis RED;

Oxford Diffraction, 2009)

$0.951,0.995$

$5883,3454,1343$

Oxford Diffraction Xcalibur with Sapphire CCD

Multi-scan (CrysAlis RED;

Oxford Diffraction, 2009)

$0.536,0.719$

$6176,3818,2063$

0.046

0.618

0.041

0.607

0.018

0.629

$0.066,0.128,1.01$

$0.065,0.135,0.94$

3454

$0.068,0.197,1.06$

3477

265

17

265

17

3818

265

17

$\mathrm{H}$ atoms treated by a mixture $\mathrm{H}$ atoms treated by a mixture of independent and constrained refinement

of independent and

constrained refinement

$0.13,-0.14$

$0.24,-0.23$

toms treated by a mixture of independent and

constrained refinement $0.94,-0.64$
(V)

(VI)

$\mathrm{C}_{11} \mathrm{H}_{17} \mathrm{~N}_{2} \mathrm{O}^{+} \cdot \mathrm{C}_{7} \mathrm{H}_{5} \mathrm{O}_{3}{ }^{-}$

330.38

Orthorhombic, $P 2_{1} 2_{1} 2_{1}$

296

6.5009 (8), 7.9735 (9), 32.155 (4)

$90,90,90$

1666.8 (3)

4

$V\left(\AA^{3}\right)$

Radiation type

$\mu\left(\mathrm{mm}^{-1}\right)$

Crystal size (mm)

Mo $K \alpha$

0.09

$0.42 \times 0.42 \times 0.34$

Oxford Diffraction Xcalibur with Sapphire CCD

Diffractometer

Absorption correction

$T_{\min }, T_{\max }$

Multi-scan (CrysAlis RED;

Oxford Diffraction, 2009)

$0.899,0.969$

No. of measured, independent $6249,3564,2875$ and observed $[I>2 \sigma(I)]$ reflections

$R_{\text {int }}$

$(\sin \theta / \lambda)_{\max }\left(\AA^{-1}\right)$

0.014

0.656

$0.041,0.089,1.05$

3564

228

0

296

0.09

0.028

0.658

3593

215

0

\section{$\mathrm{C}_{11} \mathrm{H}_{17} \mathrm{~N}_{2} \mathrm{O}^{+} \cdot \mathrm{C}_{6} \mathrm{H}_{4} \mathrm{NO}_{2}{ }^{-}$}

315.37

Orthorhombic, $\mathrm{Pbca}$

9.2817 (7), 11.2905 (7),

30.309 (2)

$90,90,90$

3176.2 (4)

Mo $K \alpha$

$0.46 \times 0.42 \times 0.36$

(VII)

$\mathrm{C}_{7} \mathrm{H}_{3} \mathrm{~N}_{2} \mathrm{O}_{7}^{+} \cdot \mathrm{C}_{11} \mathrm{H}_{17} \mathrm{~N}_{2} \mathrm{O}^{-}$

420.38

Monoclinic, $P 2_{1} / c$

296

7.5500 (9), 7.6489 (9),

32.719 (6)

90, $91.30(1), 90$

$1889.0(5)$

Mo $K \alpha$

0.12

$0.18 \times 0.12 \times 0.06$

(VIII)

Oxford Diffraction Xcalibur with Sapphire CCD

Multi-scan (CrysAlis RED;

Oxford Diffraction, 2009)

$0.879,0.968$

22154, 3593, 2616

$0.048,0.119,1.04$
Oxford Diffraction Xcalibur with Sapphire CCD

Multi-scan (CrysAlis RED;

Oxford Diffraction, 2009)

$0.916,0.993$

$8215,4074,2003$

0.038

0.660

$0.066,0.128,1.03$

4074

281

0
$\mathrm{C}_{11} \mathrm{H}_{17} \mathrm{~N}_{2} \mathrm{O}^{+} \cdot \mathrm{C}_{4} \mathrm{H}_{5} \mathrm{O}_{4}$

310.35

Orthorhombic, Pna $2_{1}$

296

9.3225 (9), $28.261(3)$,

$5.8228(8)$

$90,90,90$

1534.1 (3)

Mo $K \alpha$

0.10

$0.44 \times 0.42 \times 0.24$

Oxford Diffraction Xcalibur with Sapphire CCD

Multi-scan (CrysAlis RED;

Oxford Diffraction, 2009)

$0.816,0.976$

5828, 2419, 2053

0.018

0.649

$0.043,0.104,1.14$

2419

233

16 
Table 2 (continued)

$\begin{array}{llll}\text { (V) } & \text { (VI) } & \text { (VII) }\end{array}$

H-atom treatment

$\mathrm{H}$ atoms treated by a mixture of independent and constrained refinement

$\mathrm{H}$ atoms treated by a mixture

$\mathrm{H}$ atoms treated by a mixture

$\mathrm{H}$ atoms treated by a mixture $0.14,-0.13$

of independent and of independent and constrained refinement constrained refinement

$\Delta \rho_{\max }, \Delta \rho_{\min }\left(\mathrm{e} \AA^{-3}\right)$

$0.19,-0.16$ $0.22,-0.23$ of independent and constrained refinement Absolute structure

Flack $x$ determined using 1011 $0.16,-0.24$ quotients $\left[\left(I^{+}\right)-\left(I^{-}\right)\right] /$ $\left[\left(I^{+}\right)+\left(I^{-}\right)\right]$(Parsons et al., 2013

Absolute structure parameter -

Flack $x$ determined using 460 quotients $\left[\left(I^{+}\right)-\left(I^{-}\right)\right] /$ $\left[\left(I^{+}\right)+\left(I^{-}\right)\right]$(Parsons et al., 2013)

\begin{tabular}{|c|c|c|c|c|}
\hline & (IX) & $(\mathrm{X})$ & $(\mathrm{XI})$ & (XII) \\
\hline \multicolumn{5}{|l|}{ Crystal data } \\
\hline Chemical formula & $\mathrm{C}_{11} \mathrm{H}_{17} \mathrm{~N}_{2} \mathrm{O}^{+} \cdot \mathrm{C}_{4} \mathrm{H}_{3} \mathrm{O}_{4}^{-}$ & $\mathrm{C}_{11} \mathrm{H}_{17} \mathrm{~N}_{2} \mathrm{O}^{+} \cdot \mathrm{C}_{4} \mathrm{H}_{3} \mathrm{O}_{4}^{-}$ & $\mathrm{C}_{11} \mathrm{H}_{17} \mathrm{~N}_{2} \mathrm{O}^{+} \cdot \mathrm{C}_{2} \mathrm{Cl}_{3} \mathrm{O}_{2}^{-}$ & $\begin{array}{l}\mathrm{C}_{11} \mathrm{H}_{17} \mathrm{~N}_{2} \mathrm{O}^{+} \cdot 0.5 \mathrm{C}_{6} \mathrm{Cl}_{2} \mathrm{O}_{4}{ }^{2-} \cdot . \\
\quad \mathrm{H}_{2} \mathrm{O}\end{array}$ \\
\hline$M_{\mathrm{r}}$ & 308.33 & 308.33 & 355.64 & 314.76 \\
\hline Crystal system, space group & Orthorhombic, Pna $2_{1}$ & Monoclinic, $P 2_{1} / c$ & Orthorhombic, $\mathrm{Pca}_{1}$ & Monoclinic, $P 2_{1} / n$ \\
\hline Temperature $(\mathrm{K})$ & 296 & 296 & 296 & 296 \\
\hline$a, b, c(\AA)$ & $9.069(1), 28.528(3), 5.8375$ (9) & $9.063(1), 6.4956(9), 26.093$ (3) & $\begin{array}{l}10.6117(11), 13.808(1), \\
10.9137(8)\end{array}$ & $\begin{array}{l}9.1597(5), 15.1434(8), \\
10.8742(6)\end{array}$ \\
\hline$\alpha, \beta, \gamma\left({ }^{\circ}\right)$ & $90,90,90$ & $90,93.18(1), 90$ & $90,90,90$ & $90,102.067(5), 90$ \\
\hline$V\left(\AA^{3}\right)$ & $1510.3(3)$ & $1533.7(3)$ & $1599.1(2)$ & $1475.02(14)$ \\
\hline$Z$ & 4 & 4 & 4 & 4 \\
\hline Radiation type & Mo $K \alpha$ & Mo $K \alpha$ & Mo $K \alpha$ & Mo $K \alpha$ \\
\hline$\mu\left(\mathrm{mm}^{-1}\right)$ & 0.10 & 0.10 & 0.58 & 0.28 \\
\hline Crystal size $(\mathrm{mm})$ & $0.48 \times 0.48 \times 0.08$ & $0.48 \times 0.44 \times 0.32$ & $0.48 \times 0.48 \times 0.20$ & $0.44 \times 0.24 \times 0.20$ \\
\hline \multicolumn{5}{|l|}{ Data collection } \\
\hline Diffractometer & $\begin{array}{l}\text { Oxford Diffraction Xcalibur } \\
\text { with Sapphire CCD }\end{array}$ & $\begin{array}{l}\text { Oxford Diffraction Xcalibur } \\
\text { with Sapphire CCD }\end{array}$ & $\begin{array}{l}\text { Oxford Diffraction Xcalibur } \\
\text { with Sapphire CCD }\end{array}$ & $\begin{array}{l}\text { Oxford Diffraction Xcalibur } \\
\text { with Sapphire CCD }\end{array}$ \\
\hline Absorption correction & $\begin{array}{l}\text { Multi-scan (CrysAlis RED; } \\
\text { Oxford Diffraction, 2009) }\end{array}$ & $\begin{array}{l}\text { Multi-scan (CrysAlis RED; } \\
\text { Oxford Diffraction, 2009) }\end{array}$ & $\begin{array}{l}\text { Multi-scan (CrysAlis RED; } \\
\text { Oxford Diffraction, 2009) }\end{array}$ & $\begin{array}{l}\text { Multi-scan (CrysAlis RED; } \\
\text { Oxford Diffraction, 2009) }\end{array}$ \\
\hline$T_{\min }, T_{\max }$ & $0.888,0.992$ & $0.871,0.968$ & $0.476,0.892$ & $0.892,0.947$ \\
\hline $\begin{array}{l}\text { No. of measured, independent } \\
\text { and observed }[I>2 \sigma(I)] \\
\text { reflections }\end{array}$ & $5834,2827,2316$ & $6112,3311,2459$ & $6173,2428,2278$ & $9650,9650,7444$ \\
\hline$R_{\text {int }}$ & 0.015 & 0.014 & 0.027 & $?$ \\
\hline$(\sin \theta / \lambda)_{\max }\left(\AA^{-1}\right)$ & 0.650 & 0.651 & 0.654 & 0.651 \\
\hline \multicolumn{5}{|l|}{ Refinement } \\
\hline$R\left[F^{2}>2 \sigma\left(F^{2}\right)\right], w R\left(F^{2}\right), S$ & $0.041,0.101,1.05$ & $0.040,0.111,1.05$ & $0.032,0.086,1.08$ & $0.039,0.105,1.02$ \\
\hline No. of reflections & 2827 & 3311 & 2428 & 9650 \\
\hline No. of parameters & 221 & 210 & 198 & 204 \\
\hline No. of restraints & 11 & 0 & 1 & 0 \\
\hline $\mathrm{H}$-atom treatment & $\begin{array}{l}\mathrm{H} \text { atoms treated by a mixture } \\
\text { of independent and } \\
\text { constrained refinement }\end{array}$ & $\begin{array}{l}\mathrm{H} \text { atoms treated by a mixture } \\
\text { of independent and } \\
\text { constrained refinement }\end{array}$ & $\begin{array}{l}\mathrm{H} \text { atoms treated by a mixture } \\
\text { of independent and } \\
\text { constrained refinement }\end{array}$ & $\begin{array}{l}\mathrm{H} \text { atoms treated by a mixture } \\
\text { of independent and } \\
\text { constrained refinement }\end{array}$ \\
\hline$\Delta \rho_{\max }, \Delta \rho_{\min }\left(\mathrm{e} \AA^{-3}\right)$ & $0.15,-0.14$ & $0.21,-0.13$ & $0.25,-0.31$ & $0.23,-0.32$ \\
\hline Absolute structure & $\begin{array}{l}\text { Flack } x \text { determined using } 769 \\
\text { quotients }\left[\left(I^{+}\right)-\left(I^{-}\right)\right] / \\
{\left[\left(I^{+}\right)+\left(I^{-}\right)\right](\text {Parsons } \text { et al., }} \\
\text { 2013) }\end{array}$ & - & $\begin{array}{l}\text { Classical Flack method } \\
\text { preferred over Parsons } \\
\text { because s.u. lower }\end{array}$ & - \\
\hline Absolute structure parameter & - & - & $0.11(7)$ & - \\
\hline
\end{tabular}

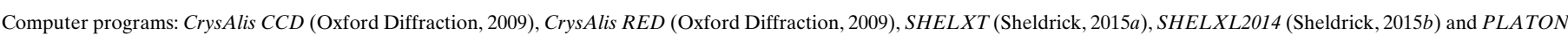
(Spek, 2009).

\section{Funding information}

HSY thanks the University Grants Commission, New Delhi, for the award of a BSR Faculty Fellowship for three years, and HKK thanks the UGC-BSR for a stipend.

\section{References}

Acosta, L. M., Bahsas, A., Palma, A., Cobo, J., Hursthouse, M. B. \& Glidewell, C. (2009). Acta Cryst. C65, o92-o96.
Allen, F. H., Kennard, O., Watson, D. G., Brammer, L., Orpen, A. G. \& Taylor, R. (1987). J. Chem. Soc. Perkin Trans. 2, pp. S1-S19.

Al-Omary, F. A. M., Ghabbour, H. A., El-Emam, A. A., Chidan Kumar, C. S. \& Fun, H.-K. (2014). Acta Cryst. E70, o245-o246.

Arbo, M. D., Bastos, M. L. \& Carmo, H. F. (2012). Drug Alcohol Depend. 122, 174-185.

Asif, M. (2015). Int. J. Adv. Sci. Res. 1, 5-11.

Bernstein, J., Davis, R. E., Shimoni, L. \& Chang, N.-L. (1995). Angew. Chem. Int. Ed. Engl. 34, 1555-1573.

Brito, A., Moreira, L. K. S., Menegatti, R. \& Costa, E. A. (2019). Fundam. Clin. Pharmacol. 33, 13-24.

Emsley, J. (1980). Chem. Soc. Rev. 9, 91-124. 
Etter, M. C. (1990). Acc. Chem. Res. 23, 120-126.

Etter, M. C., MacDonald, J. C. \& Bernstein, J. (1990). Acta Cryst. B46, 256-262.

Ferguson, G., Glidewell, C., Gregson, R. M. \& Meehan, P. R. (1998a). Acta Cryst. B54, 129-138.

Ferguson, G., Glidewell, C., Gregson, R. M. \& Meehan, P. R. (1998b). Acta Cryst. B54, 139-150.

Flack, H. D. (1983). Acta Cryst. A39, 876-881.

Flack, H. D. \& Bernardinelli, G. (2000). J. Appl. Cryst. 33, 1143-1148.

Glidewell, C., Low, J. N., Skakle, J. M. S. \& Wardell, J. L. (2005). Acta Cryst. C61, o276-o280.

Gregson, R. M., Glidewell, C., Ferguson, G. \& Lough, A. J. (2000). Acta Cryst. B56, 39-57.

Herschlag, D. \& Pinney, M. M. (2018). Biochemistry, 57, 3338-3352.

Ishida, H. (2004a). Acta Cryst. E60, o974-0976.

Ishida, H. (2004b). Acta Cryst. E60, o1900-o1901.

Ishida, H. (2004c). Acta Cryst. E60, o2005-o2006.

Ishida, H. (2004d). Acta Cryst. E60, o2506-o2508.

Kaur, M., Jasinski, J. P., Yathirajan, H. S., Kavitha, C. N. \& Glidewell, C. (2015). Acta Cryst. E71, 406-413.

Kavitha, C. N., Jasinski, J. P., Anderson, B. J., Yathirajan, H. S. \& Kaur, M. (2013). Acta Cryst. E69, o1671.

Kavitha, C. N., Yathirajan, H. S., Kaur, M., Hosten, E. C., Betz, R. \& Glidewell, C. (2014). Acta Cryst. C70, 805-811.

Kavitha, S. J., Panchanatheswaran, K., Low, J. N., Ferguson, G. \& Glidewell, C. (2006). Acta Cryst. C62, o165-o169.

Kiran Kumar, H., Yathirajan, H. S., Sagar, B. K., Foro, S. \& Glidewell, C. (2019). Acta Cryst. E75, 1253-1260.

Lu, Y.-X. (2007). Acta Cryst. E63, o3611.

Nagai, F., Nonaka, R. \& Kamimura, K. S. H. (2007). Eur. J. Pharmacol. 559, 132-137.
Oxford Diffraction (2009). CrysAlis CCD and CrysAlis RED. Oxford Diffraction Ltd, Abingdon, England.

Parsons, S., Flack, H. D. \& Wagner, T. (2013). Acta Cryst. B69, 249259.

Sagar, B. K., Girisha, M., Yathirajan, H. S., Rathore, R. S. \& Glidewell, C. (2017). Acta Cryst. E73, 1320-1325.

Shaibah, M. A. E., Sagar, B. K., Yathirajan, H. S., Kumar, S. M. \& Glidewell, C. (2017a). Acta Cryst. E73, 1513-1516.

Shaibah, M. A. E., Yathirajan, H. S., Kumar, S. M., Byrappa, K. \& Glidewell, C. (2017b). Acta Cryst. E73, 1488-1492.

Shaibah, M. A. E., Yathirajan, H. S., Rathore, R. S., Furuya, T., Haraguchi, T., Akitsu, T. \& Glidewell, C. (2019). Acta Cryst. E75, 292-298.

Sheldrick, G. M. (2015a). Acta Cryst. A71, 3-8.

Sheldrick, G. M. (2015b). Acta Cryst. C71, 3-8.

Sovago, I., Thomas, L. H., Adam, M. S., Capelli, S. C., Wilson, C. C. \& Farrugia, L. J. (2016). CrystEngComm, 18, 5697-5709.

Spek, A. L. (2009). Acta Cryst. D65, 148-155.

Staack, R. F. \& Maurer, H. H. (2003). J. Chromatogr. B, 798, 333-342.

Staack, R. F. \& Maurer, H. H. (2005). Curr. Drug Metab. 6, 259-274.

Staack, R. F., Theobald, D. S., Paul, D., Springer, D., Kraemer, T. \& Maurer, H. H. (2004). Xenobiotica, 34, 179-192.

Verdonk, M. L., Voogd, J. W., Kanters, J. A., Kroon, J., den Besten, R., Brandsma, L., Leysen, D. \& Kelder, J. (1997). Acta Cryst. B53, 976983.

Wohlfarth, A., Weinmann, W. \& Dresen, S. (2010). Anal. Bioanal. Chem. 396, 2403-2414.

Wood, P. A., Allen, F. H. \& Pidcock, E. (2009). CrystEngComm, 11, 1563-1571.

Zia-ur-Rehman, Tahir, M. N., Danish, M., Muhammad, N. \& Ali, S. (2009). Acta Cryst. E65, o503. 


\section{supporting information}

Acta Cryst. (2019). E75, 1494-1506 [https://doi.org/10.1107/S2056989019012702]

\section{Twelve 4-(4-methoxyphenyl)piperazin-1-ium salts containing organic anions:}

\section{supramolecular assembly in one, two and three dimensions}

\section{Haruvegowda Kiran Kumar, Hemmige S. Yathirajan, Sabine Foro and Christopher Glidewell}

Computing details

For all structures, data collection: CrysAlis CCD (Oxford Diffraction, 2009); cell refinement: CrysAlis RED (Oxford Diffraction, 2009); data reduction: CrysAlis RED (Oxford Diffraction, 2009); program(s) used to solve structure:

SHELXT (Sheldrick, 2015a); program(s) used to refine structure: SHELXL2014 (Sheldrick, 2015b); molecular graphics: PLATON (Spek, 2009); software used to prepare material for publication: SHELXL2014 (Sheldrick, 2015b) and PLATON (Spek, 2009).

4-(4-Methoxyphenyl)piperazin-1-ium benzoate monohydrate (I)

Crystal data

$\mathrm{C}_{11} \mathrm{H}_{17} \mathrm{~N}_{2} \mathrm{O}^{+} \cdot \mathrm{C}_{7} \mathrm{H}_{5} \mathrm{O}_{2}^{-} \cdot \mathrm{H}_{2} \mathrm{O}$

$M_{r}=332.39$

Triclinic, $P \overline{1}$

$a=6.215(1) \AA$

$b=7.547(1) \AA$

$c=18.716(4) \AA$

$\alpha=84.34(2)^{\circ}$

$\beta=87.14(2)^{\circ}$

$\gamma=84.69(2)^{\circ}$

$V=869.1(3) \AA^{3}$

\section{Data collection}

Oxford Diffraction Xcalibur with Sapphire CCD diffractometer

Radiation source: Enhance (Mo) X-ray Source

Graphite monochromator

$\omega$ scans

Absorption correction: multi-scan

(CrysAlis RED; Oxford Diffraction, 2009)

$T_{\min }=0.834, T_{\max }=0.996$

\section{Refinement}

Refinement on $F^{2}$

Least-squares matrix: full

$R\left[F^{2}>2 \sigma\left(F^{2}\right)\right]=0.054$

$w R\left(F^{2}\right)=0.134$

$S=1.02$

3442 reflections

256 parameters
$Z=2$

$F(000)=356$

$D_{\mathrm{x}}=1.270 \mathrm{Mg} \mathrm{m}^{-3}$

Mo $K \alpha$ radiation, $\lambda=0.71073 \AA$

Cell parameters from 3742 reflections

$\theta=2.8-28.0^{\circ}$

$\mu=0.09 \mathrm{~mm}^{-1}$

$T=296 \mathrm{~K}$

Plate, colourless

$0.40 \times 0.24 \times 0.04 \mathrm{~mm}$

5751 measured reflections

3442 independent reflections

1839 reflections with $I>2 \sigma(I)$

$R_{\text {int }}=0.029$

$\theta_{\max }=26.1^{\circ}, \theta_{\min }=2.8^{\circ}$

$h=-7 \rightarrow 7$

$k=-9 \rightarrow 9$

$l=-19 \rightarrow 23$

17 restraints

Primary atom site location: difference Fourier map

Hydrogen site location: mixed

$\mathrm{H}$ atoms treated by a mixture of independent and constrained refinement 
$w=1 /\left[\sigma^{2}\left(F_{\mathrm{o}}^{2}\right)+(0.0611 P)^{2}\right]$

where $P=\left(F_{\mathrm{o}}^{2}+2 F_{\mathrm{c}}^{2}\right) / 3$

$(\Delta / \sigma)_{\max }<0.001$

$$
\Delta \rho_{\max }=0.17 \mathrm{e} \AA^{-3}
$$

$\Delta \rho_{\min }=-0.19$ e $\AA^{-3}$

Special details

Experimental. Compound (I). IR $\left(\mathrm{KBr}, \mathrm{cm}^{-1}\right) 3328(\mathrm{OH}), 3002\left(\mathrm{H}_{2}\right) 2841\left(\mathrm{OCH}_{3}\right), 1591(\mathrm{COO})$. NMR $\left(\mathrm{CDCl}_{3}\right) \delta\left({ }^{1} \mathrm{H}\right)$ $3.22\left(\mathrm{~m}, 4 \mathrm{H}\right.$, piperazine), $3.29\left(\mathrm{~m}, 4 \mathrm{H}\right.$, piperazine), $3.77\left(\mathrm{~s}, 3 \mathrm{H}, \mathrm{OCH}_{3}\right), 6.86(\mathrm{~m}, 4 \mathrm{H}$, methoxyphenyl), $7.39(\mathrm{~m}, 2 \mathrm{H}$, phenyl), 7.46 (m, 1H, phenyl), 8.05 (m, 2H, phenyl).

Geometry. All esds (except the esd in the dihedral angle between two 1.s. planes) are estimated using the full covariance matrix. The cell esds are taken into account individually in the estimation of esds in distances, angles and torsion angles; correlations between esds in cell parameters are only used when they are defined by crystal symmetry. An approximate (isotropic) treatment of cell esds is used for estimating esds involving 1.s. planes.

Fractional atomic coordinates and isotropic or equivalent isotropic displacement parameters $\left(\AA^{2}\right)$

\begin{tabular}{|c|c|c|c|c|c|}
\hline & $x$ & $y$ & $z$ & $U_{\text {iso }} * / U_{\text {eq }}$ & Occ. $(<1)$ \\
\hline N1 & $0.7874(3)$ & 0.7435 & $0.44989(10)$ & $0.0482(5)$ & \\
\hline H11 & $0.834(3)$ & $0.766(3)$ & $0.4926(13)$ & $0.058^{*}$ & \\
\hline $\mathrm{H} 12$ & $0.748(3)$ & $0.621(3)$ & $0.4555(11)$ & $0.058^{*}$ & \\
\hline $\mathrm{C} 2$ & $0.9747(3)$ & 0.7530 & 0.39809 (12) & $0.0519(6)$ & \\
\hline $\mathrm{H} 2 \mathrm{~A}$ & 1.0940 & 0.6709 & 0.4157 & $0.062 *$ & \\
\hline $\mathrm{H} 2 \mathrm{~B}$ & 1.0215 & 0.8729 & 0.3934 & $0.062 *$ & \\
\hline $\mathrm{C} 3$ & $0.9144(3)$ & 0.7056 & $0.32596(12)$ & $0.0497(6)$ & \\
\hline $\mathrm{H} 3 \mathrm{~A}$ & 1.0363 & 0.7191 & 0.2921 & $0.060 *$ & \\
\hline H3B & 0.8837 & 0.5812 & 0.3301 & $0.060 *$ & \\
\hline N4 & $0.7262(2)$ & $0.8168(2)$ & $0.29814(9)$ & $0.0394(4)$ & \\
\hline $\mathrm{C} 5$ & 0.5425 & 0.8133 & $0.35024(11)$ & $0.0463(5)$ & \\
\hline $\mathrm{H} 5 \mathrm{~A}$ & 0.4932 & 0.6943 & 0.3561 & $0.056^{*}$ & \\
\hline H5B & 0.4244 & 0.8957 & 0.3320 & $0.056^{*}$ & \\
\hline C6 & $0.6019(3)$ & 0.8640 & $0.42214(12)$ & $0.0513(6)$ & \\
\hline H6A & 0.6397 & 0.9866 & 0.4173 & $0.062 *$ & \\
\hline H6B & 0.4789 & 0.8557 & 0.4559 & $0.062 *$ & \\
\hline $\mathrm{C} 21$ & $0.677(2)$ & $0.782(2)$ & $0.2275(6)$ & $0.034(2)$ & $0.66(2)$ \\
\hline $\mathrm{C} 22$ & $0.8134(13)$ & $0.6810(17)$ & $0.1831(5)$ & $0.0450(18)$ & $0.66(2)$ \\
\hline $\mathrm{H} 22$ & 0.9414 & 0.6235 & 0.2007 & $0.054 *$ & $0.66(2)$ \\
\hline $\mathrm{C} 23$ & $0.7627(13)$ & $0.6649(18)$ & $0.1132(5)$ & $0.053(2)$ & $0.66(2)$ \\
\hline $\mathrm{H} 23$ & 0.8602 & 0.6005 & 0.0841 & $0.064 *$ & $0.66(2)$ \\
\hline $\mathrm{C} 24$ & $0.5725(14)$ & $0.7413(16)$ & $0.0858(5)$ & $0.0438(17)$ & $0.66(2)$ \\
\hline $\mathrm{C} 25$ & $0.438(2)$ & $0.846(3)$ & $0.1278(8)$ & $0.0599(10)$ & $0.66(2)$ \\
\hline $\mathrm{H} 25$ & 0.3145 & 0.9097 & 0.1088 & $0.072 *$ & $0.66(2)$ \\
\hline $\mathrm{C} 26$ & $0.486(3)$ & $0.858(3)$ & $0.1984(8)$ & $0.054(2)$ & $0.66(2)$ \\
\hline H26 & 0.3857 & 0.9191 & 0.2276 & $0.064 *$ & $0.66(2)$ \\
\hline $\mathrm{O} 24$ & $0.541(3)$ & $0.720(3)$ & $0.0149(7)$ & $0.078(3)$ & $0.66(2)$ \\
\hline $\mathrm{C} 27$ & $0.335(3)$ & $0.773(4)$ & $-0.0122(11)$ & $0.090(2)$ & $0.66(2)$ \\
\hline $\mathrm{H} 27 \mathrm{~A}$ & 0.2297 & 0.7017 & 0.0128 & $0.135^{*}$ & $0.66(2)$ \\
\hline $\mathrm{H} 27 \mathrm{~B}$ & 0.3373 & 0.7580 & -0.0626 & $0.135^{*}$ & $0.66(2)$ \\
\hline $\mathrm{H} 27 \mathrm{C}$ & 0.2962 & 0.8970 & -0.0053 & $0.135 *$ & $0.66(2)$ \\
\hline C51 & $0.669(5)$ & $0.815(5)$ & $0.2258(12)$ & $0.034(2)$ & $0.34(2)$ \\
\hline C52 & $0.817(3)$ & $0.735(2)$ & 0.1784 (11) & $0.0450(18)$ & $0.34(2)$ \\
\hline
\end{tabular}




\begin{tabular}{|c|c|c|c|c|c|}
\hline H52 & 0.9535 & 0.6917 & 0.1937 & $0.054 *$ & $0.34(2)$ \\
\hline C53 & $0.764(3)$ & $0.718(3)$ & $0.1087(10)$ & $0.053(2)$ & $0.34(2)$ \\
\hline H53 & 0.8615 & 0.6555 & 0.0790 & $0.064 *$ & $0.34(2)$ \\
\hline C54 & $0.571(3)$ & $0.792(2)$ & $0.0828(10)$ & $0.0438(17)$ & $0.34(2)$ \\
\hline C55 & $0.414(4)$ & $0.854(7)$ & $0.1309(15)$ & $0.0599(10)$ & $0.34(2)$ \\
\hline H55 & 0.2702 & 0.8738 & 0.1184 & $0.072 *$ & $0.34(2)$ \\
\hline C56 & $0.474(5)$ & $0.886(7)$ & $0.1983(15)$ & $0.054(2)$ & $0.34(2)$ \\
\hline H56 & 0.3803 & 0.9570 & 0.2260 & $0.064^{*}$ & $0.34(2)$ \\
\hline O54 & $0.528(6)$ & $0.756(7)$ & $0.0142(14)$ & $0.078(3)$ & $0.34(2)$ \\
\hline C57 & $0.310(6)$ & $0.780(9)$ & $-0.006(2)$ & $0.090(2)$ & $0.34(2)$ \\
\hline H57A & 0.2919 & 0.7103 & -0.0451 & $0.135^{*}$ & $0.34(2)$ \\
\hline H57B & 0.2718 & 0.9042 & -0.0207 & $0.135^{*}$ & $0.34(2)$ \\
\hline $\mathrm{H} 57 \mathrm{C}$ & 0.2171 & 0.7422 & 0.0342 & $0.135^{*}$ & $0.34(2)$ \\
\hline C31 & $0.8112(3)$ & $0.7289(3)$ & $0.70824(12)$ & $0.0441(5)$ & \\
\hline C32 & 0.6694 (4) & $0.6501(3)$ & 0.75905 (17) & $0.0642(7)$ & \\
\hline H32 & 0.5518 & 0.5977 & 0.7441 & $0.077 *$ & \\
\hline C33 & $0.7024(5)$ & $0.6491(3)$ & $0.83138(17)$ & $0.0777(9)$ & \\
\hline H33 & 0.6070 & 0.5956 & 0.8649 & $0.093 *$ & \\
\hline C34 & $0.8735(5)$ & $0.7259(3)$ & $0.85422(15)$ & $0.0741(8)$ & \\
\hline H34 & 0.8935 & 0.7264 & 0.9031 & $0.089 *$ & \\
\hline C35 & 1.0147 (4) & $0.8017(3)$ & $0.80535(14)$ & $0.0631(7)$ & \\
\hline H35 & 1.1322 & 0.8530 & 0.8209 & $0.076^{*}$ & \\
\hline C36 & 0.9850 & $0.8032(3)$ & $0.73275(13)$ & $0.0483(6)$ & \\
\hline H36 & 1.0835 & 0.8550 & 0.6999 & $0.058^{*}$ & \\
\hline C37 & $0.7750(4)$ & $0.7325(3)$ & $0.62925(15)$ & $0.0563(6)$ & \\
\hline O31 & 0.9017 (3) & $0.8122(2)$ & $0.58536(9)$ & $0.0664(5)$ & \\
\hline O32 & $0.6242(3)$ & $0.6520(3)$ & $0.61161(12)$ & $0.1072(8)$ & \\
\hline O41 & 0.7231 & $0.3783(2)$ & $0.45956(10)$ & $0.0657(5)$ & \\
\hline H41 & $0.607(5)$ & $0.372(4)$ & $0.4354(16)$ & $0.099 *$ & \\
\hline $\mathrm{H} 42$ & $0.839(5)$ & $0.318(4)$ & $0.4391(16)$ & $0.099 *$ & \\
\hline
\end{tabular}

Atomic displacement parameters $\left(\AA^{2}\right)$

\begin{tabular}{lllllll}
\hline & $U^{11}$ & $U^{22}$ & $U^{33}$ & $U^{12}$ & $U^{13}$ & $U^{23}$ \\
\hline $\mathrm{N} 1$ & $0.0614(13)$ & $0.0460(11)$ & $0.0394(12)$ & $-0.0089(9)$ & $-0.0119(10)$ & $-0.0069(9)$ \\
$\mathrm{C} 2$ & $0.0452(14)$ & $0.0584(14)$ & $0.0529(16)$ & $-0.0018(11)$ & $-0.0116(11)$ & $-0.0075(12)$ \\
$\mathrm{C} 3$ & $0.0424(13)$ & $0.0583(14)$ & $0.0483(15)$ & $0.0016(10)$ & $-0.0047(10)$ & $-0.0084(11)$ \\
$\mathrm{N} 4$ & $0.0391(10)$ & $0.0403(10)$ & $0.0386(11)$ & $0.0002(7)$ & $-0.0018(8)$ & $-0.0064(8)$ \\
C5 & $0.0414(12)$ & $0.0553(13)$ & $0.0413(14)$ & $0.0022(10)$ & $-0.0001(10)$ & $-0.0072(10)$ \\
C6 & $0.0561(14)$ & $0.0529(14)$ & $0.0438(15)$ & $0.0044(11)$ & $-0.0012(11)$ & $-0.0083(11)$ \\
C21 & $0.0428(15)$ & $0.022(6)$ & $0.0377(14)$ & $-0.001(3)$ & $-0.0009(10)$ & $-0.002(2)$ \\
C22 & $0.0449(14)$ & $0.041(5)$ & $0.048(2)$ & $0.005(3)$ & $-0.0021(13)$ & $-0.007(3)$ \\
C23 & $0.0575(16)$ & $0.053(6)$ & $0.048(2)$ & $0.008(3)$ & $0.0060(13)$ & $-0.015(3)$ \\
C24 & $0.0628(16)$ & $0.031(5)$ & $0.0378(17)$ & $-0.004(3)$ & $-0.0012(13)$ & $-0.002(3)$ \\
C25 & $0.057(3)$ & $0.072(2)$ & $0.047(2)$ & $0.020(3)$ & $-0.013(2)$ & $-0.0097(16)$ \\
C26 & $0.057(2)$ & $0.054(7)$ & $0.0477(16)$ & $0.022(2)$ & $-0.0054(14)$ & $-0.014(3)$ \\
O24 & $0.083(2)$ & $0.110(10)$ & $0.0410(12)$ & $0.009(3)$ & $-0.0107(11)$ & $-0.023(3)$ \\
C27 & $0.081(5)$ & $0.140(3)$ & $0.052(3)$ & $-0.008(4)$ & $-0.018(4)$ & $-0.020(4)$
\end{tabular}




$\begin{array}{lllllll}\text { C51 } & 0.0428(15) & 0.022(6) & 0.0377(14) & -0.001(3) & -0.0009(10) & -0.002(2) \\ \text { C52 } & 0.0449(14) & 0.041(5) & 0.048(2) & 0.005(3) & -0.0021(13) & -0.007(3) \\ \text { C53 } & 0.0575(16) & 0.053(6) & 0.048(2) & 0.008(3) & 0.0060(13) & -0.015(3) \\ \text { C54 } & 0.0628(16) & 0.031(5) & 0.0378(17) & -0.004(3) & -0.0012(13) & -0.002(3) \\ \text { C55 } & 0.057(3) & 0.072(2) & 0.047(2) & 0.020(3) & -0.013(2) & -0.0097(16) \\ \text { C56 } & 0.057(2) & 0.054(7) & 0.0477(16) & 0.022(2) & -0.0054(14) & -0.014(3) \\ \text { O54 } & 0.083(2) & 0.110(10) & 0.0410(12) & 0.009(3) & -0.0107(11) & -0.023(3) \\ \text { C57 } & 0.081(5) & 0.140(3) & 0.052(3) & -0.008(4) & -0.018(4) & -0.020(4) \\ \text { C31 } & 0.0428(12) & 0.0353(11) & 0.0550(15) & 0.0023(10) & -0.0041(11) & -0.0117(10) \\ \text { C32 } & 0.0510(15) & 0.0506(15) & 0.092(2) & -0.0061(11) & 0.0098(14) & -0.0174(14) \\ \text { C33 } & 0.091(2) & 0.0614(17) & 0.073(2) & 0.0018(15) & 0.0343(17) & 0.0038(15) \\ \text { C34 } & 0.099(2) & 0.0639(17) & 0.0554(19) & 0.0101(16) & -0.0013(17) & -0.0025(14) \\ \text { C35 } & 0.0746(18) & 0.0624(16) & 0.0530(18) & -0.0031(13) & -0.0179(14) & -0.0044(13) \\ \text { C36 } & 0.0509(13) & 0.0454(13) & 0.0492(16) & -0.0057(10) & -0.0079(11) & -0.0023(10) \\ \text { C37 } & 0.0540(15) & 0.0504(15) & 0.0680(19) & 0.0062(12) & -0.0207(13) & -0.0233(13) \\ \text { O31 } & 0.0856(13) & 0.0687(11) & 0.0468(11) & -0.0072(10) & -0.0138(9) & -0.0092(9) \\ \text { O32 } & 0.0869(14) & 0.1422(19) & 0.1065(18) & -0.0380(13) & -0.0368(12) & -0.0380(15) \\ \text { O41 } & 0.0653(12) & 0.0631(11) & 0.0715(14) & -0.0142(9) & -0.0198(9) & -0.0044(9) \\ & & & & & \end{array}$

Geometric parameters $\left(\AA,{ }^{\circ}\right)$

\begin{tabular}{llll}
\hline $\mathrm{N} 1-\mathrm{C} 2$ & $1.480(3)$ & $\mathrm{C} 27-\mathrm{H} 27 \mathrm{C}$ & 0.9600 \\
$\mathrm{~N} 1-\mathrm{C} 6$ & $1.483(3)$ & $\mathrm{C} 51-\mathrm{C} 56$ & $1.381(7)$ \\
$\mathrm{N} 1-\mathrm{H} 11$ & $0.90(2)$ & $\mathrm{C} 51-\mathrm{C} 52$ & $1.385(7)$ \\
$\mathrm{N} 1-\mathrm{H} 12$ & $0.97(2)$ & $\mathrm{C} 52-\mathrm{C} 53$ & $1.380(7)$ \\
$\mathrm{C} 2-\mathrm{C} 3$ & $1.504(3)$ & $\mathrm{C} 52-\mathrm{H} 52$ & 0.9300 \\
$\mathrm{C} 2-\mathrm{H} 2 \mathrm{~A}$ & 0.9700 & $\mathrm{C} 53-\mathrm{C} 54$ & $1.368(7)$ \\
$\mathrm{C} 2-\mathrm{H} 2 \mathrm{~B}$ & 0.9700 & $\mathrm{C} 53-\mathrm{H} 53$ & 0.9300 \\
$\mathrm{C} 3-\mathrm{N} 4$ & $1.461(2)$ & $\mathrm{C} 54-\mathrm{C} 55$ & $1.374(9)$ \\
$\mathrm{C} 3-\mathrm{H} 3 \mathrm{~A}$ & 0.9700 & $\mathrm{C} 54-\mathrm{O} 54$ & $1.380(7)$ \\
$\mathrm{C} 3-\mathrm{H} 3 \mathrm{~B}$ & 0.9700 & $\mathrm{C} 55-\mathrm{C} 56$ & $1.383(9)$ \\
$\mathrm{N} 4-\mathrm{C} 51$ & $1.42(2)$ & $\mathrm{C} 55-\mathrm{H} 55$ & 0.9300 \\
$\mathrm{~N} 4-\mathrm{C} 21$ & $1.428(10)$ & $\mathrm{C} 56-\mathrm{H} 56$ & 0.9300 \\
$\mathrm{~N} 4-\mathrm{C} 5$ & $1.464(2)$ & $\mathrm{O} 54-\mathrm{C} 57$ & $1.415(9)$ \\
$\mathrm{C} 5-\mathrm{C} 6$ & $1.507(3)$ & $\mathrm{C} 57-\mathrm{H} 57 \mathrm{~A}$ & 0.9600 \\
$\mathrm{C} 5-\mathrm{H} 5 \mathrm{~A}$ & 0.9700 & $\mathrm{C} 57-\mathrm{H} 57 \mathrm{~B}$ & 0.9600 \\
$\mathrm{C} 5-\mathrm{H} 5 \mathrm{~B}$ & 0.9700 & $\mathrm{C} 57-\mathrm{H} 57 \mathrm{C}$ & 0.9600 \\
$\mathrm{C} 6-\mathrm{H} 6 \mathrm{~A}$ & 0.9700 & $\mathrm{C} 31-\mathrm{C} 36$ & $1.380(3)$ \\
$\mathrm{C} 6-\mathrm{H} 6 \mathrm{~B}$ & 0.9700 & $\mathrm{C} 31-\mathrm{C} 32$ & $1.391(3)$ \\
$\mathrm{C} 21-\mathrm{C} 26$ & $1.382(4)$ & $\mathrm{C} 31-\mathrm{C} 37$ & $1.504(3)$ \\
$\mathrm{C} 21-\mathrm{C} 22$ & $1.386(4)$ & $\mathrm{C} 32-\mathrm{C} 33$ & $1.378(4)$ \\
$\mathrm{C} 22-\mathrm{C} 23$ & $1.380(4)$ & $\mathrm{C} 32-\mathrm{H} 32$ & 0.9300 \\
$\mathrm{C} 22-\mathrm{H} 22$ & 0.9300 & $\mathrm{C} 33-\mathrm{C} 34$ & $1.363(4)$ \\
$\mathrm{C} 23-\mathrm{C} 24$ & $1.367(4)$ & $\mathrm{C} 33-\mathrm{H} 33$ & 0.9300 \\
$\mathrm{C} 23-\mathrm{H} 23$ & 0.9300 & $\mathrm{C} 34-\mathrm{C} 35$ & $1.358(3)$ \\
$\mathrm{C} 24-\mathrm{C} 25$ & $1.372(7)$ & $\mathrm{C} 34-\mathrm{H} 34$ & 0.9300 \\
$\mathrm{C} 24-\mathrm{O} 24$ & $1.378(4)$ & $\mathrm{C} 35-\mathrm{C} 36$ & $1.379(3)$ \\
$\mathrm{C} 25-\mathrm{C} 26$ & $1.382(5)$ & $\mathrm{C} 35-\mathrm{H} 35$ & 0.9300 \\
& & &
\end{tabular}




\begin{tabular}{|c|c|c|c|}
\hline $\mathrm{C} 25-\mathrm{H} 25$ & 0.9300 & $\mathrm{C} 36-\mathrm{H} 36$ & 0.9300 \\
\hline $\mathrm{C} 26-\mathrm{H} 26$ & 0.9300 & $\mathrm{C} 37-\mathrm{O} 32$ & $1.238(3)$ \\
\hline $\mathrm{O} 24-\mathrm{C} 27$ & $1.413(6)$ & $\mathrm{C} 37-\mathrm{O} 31$ & $1.258(3)$ \\
\hline $\mathrm{C} 27-\mathrm{H} 27 \mathrm{~A}$ & 0.9600 & $\mathrm{O} 41-\mathrm{H} 41$ & $0.88(3)$ \\
\hline $\mathrm{C} 27-\mathrm{H} 27 \mathrm{~B}$ & 0.9600 & $\mathrm{O} 41-\mathrm{H} 42$ & $0.91(3)$ \\
\hline $\mathrm{C} 2-\mathrm{N} 1-\mathrm{C} 6$ & $109.95(18)$ & $\mathrm{O} 24-\mathrm{C} 27-\mathrm{H} 27 \mathrm{~A}$ & 109.5 \\
\hline $\mathrm{C} 2-\mathrm{N} 1-\mathrm{H} 11$ & $106.8(14)$ & $\mathrm{O} 24-\mathrm{C} 27-\mathrm{H} 27 \mathrm{~B}$ & 109.5 \\
\hline $\mathrm{C} 6-\mathrm{N} 1-\mathrm{H} 11$ & $115.6(14)$ & $\mathrm{H} 27 \mathrm{~A}-\mathrm{C} 27-\mathrm{H} 27 \mathrm{~B}$ & 109.5 \\
\hline $\mathrm{C} 2-\mathrm{N} 1-\mathrm{H} 12$ & $108.2(12)$ & $\mathrm{O} 24-\mathrm{C} 27-\mathrm{H} 27 \mathrm{C}$ & 109.5 \\
\hline $\mathrm{C} 6-\mathrm{N} 1-\mathrm{H} 12$ & $109.2(12)$ & $\mathrm{H} 27 \mathrm{~A}-\mathrm{C} 27-\mathrm{H} 27 \mathrm{C}$ & 109.5 \\
\hline $\mathrm{H} 11-\mathrm{N} 1-\mathrm{H} 12$ & $106.8(18)$ & $\mathrm{H} 27 \mathrm{~B}-\mathrm{C} 27-\mathrm{H} 27 \mathrm{C}$ & 109.5 \\
\hline $\mathrm{N} 1-\mathrm{C} 2-\mathrm{C} 3$ & $110.23(17)$ & $\mathrm{C} 56-\mathrm{C} 51-\mathrm{C} 52$ & $116.4(9)$ \\
\hline $\mathrm{N} 1-\mathrm{C} 2-\mathrm{H} 2 \mathrm{~A}$ & 109.6 & $\mathrm{C} 56-\mathrm{C} 51-\mathrm{N} 4$ & $125.0(16)$ \\
\hline $\mathrm{C} 3-\mathrm{C} 2-\mathrm{H} 2 \mathrm{~A}$ & 109.6 & $\mathrm{C} 52-\mathrm{C} 51-\mathrm{N} 4$ & $118.6(16)$ \\
\hline $\mathrm{N} 1-\mathrm{C} 2-\mathrm{H} 2 \mathrm{~B}$ & 109.6 & $\mathrm{C} 53-\mathrm{C} 52-\mathrm{C} 51$ & $121.0(9)$ \\
\hline $\mathrm{C} 3-\mathrm{C} 2-\mathrm{H} 2 \mathrm{~B}$ & 109.6 & $\mathrm{C} 53-\mathrm{C} 52-\mathrm{H} 52$ & 119.5 \\
\hline $\mathrm{H} 2 \mathrm{~A}-\mathrm{C} 2-\mathrm{H} 2 \mathrm{~B}$ & 108.1 & $\mathrm{C} 51-\mathrm{C} 52-\mathrm{H} 52$ & 119.5 \\
\hline $\mathrm{N} 4-\mathrm{C} 3-\mathrm{C} 2$ & $112.64(17)$ & $\mathrm{C} 54-\mathrm{C} 53-\mathrm{C} 52$ & $121.0(9)$ \\
\hline $\mathrm{N} 4-\mathrm{C} 3-\mathrm{H} 3 \mathrm{~A}$ & 109.1 & $\mathrm{C} 54-\mathrm{C} 53-\mathrm{H} 53$ & 119.5 \\
\hline $\mathrm{C} 2-\mathrm{C} 3-\mathrm{H} 3 \mathrm{~A}$ & 109.1 & $\mathrm{C} 52-\mathrm{C} 53-\mathrm{H} 53$ & 119.5 \\
\hline $\mathrm{N} 4-\mathrm{C} 3-\mathrm{H} 3 \mathrm{~B}$ & 109.1 & $\mathrm{C} 53-\mathrm{C} 54-\mathrm{C} 55$ & $118.1(9)$ \\
\hline $\mathrm{C} 2-\mathrm{C} 3-\mathrm{H} 3 \mathrm{~B}$ & 109.1 & $\mathrm{C} 53-\mathrm{C} 54-\mathrm{O} 54$ & $116.3(10)$ \\
\hline $\mathrm{H} 3 \mathrm{~A}-\mathrm{C} 3-\mathrm{H} 3 \mathrm{~B}$ & 107.8 & $\mathrm{C} 55-\mathrm{C} 54-\mathrm{O} 54$ & $123.3(12)$ \\
\hline $\mathrm{C} 51-\mathrm{N} 4-\mathrm{C} 3$ & $120.7(9)$ & C54-C55-C56 & $118.8(13)$ \\
\hline $\mathrm{C} 21-\mathrm{N} 4-\mathrm{C} 3$ & $113.1(5)$ & $\mathrm{C} 54-\mathrm{C} 55-\mathrm{H} 55$ & 120.6 \\
\hline $\mathrm{C} 51-\mathrm{N} 4-\mathrm{C} 5$ & $114.2(13)$ & C56-C55-H55 & 120.6 \\
\hline $\mathrm{C} 21-\mathrm{N} 4-\mathrm{C} 5$ & $114.4(6)$ & $\mathrm{C} 51-\mathrm{C} 56-\mathrm{C} 55$ & $122.1(10)$ \\
\hline $\mathrm{C} 3-\mathrm{N} 4-\mathrm{C} 5$ & $111.20(16)$ & $\mathrm{C} 51-\mathrm{C} 56-\mathrm{H} 56$ & 119.0 \\
\hline $\mathrm{N} 4-\mathrm{C} 5-\mathrm{C} 6$ & $111.63(17)$ & $\mathrm{C} 55-\mathrm{C} 56-\mathrm{H} 56$ & 119.0 \\
\hline $\mathrm{N} 4-\mathrm{C} 5-\mathrm{H} 5 \mathrm{~A}$ & 109.3 & $\mathrm{C} 54-\mathrm{O} 54-\mathrm{C} 57$ & $117.7(12)$ \\
\hline $\mathrm{C} 6-\mathrm{C} 5-\mathrm{H} 5 \mathrm{~A}$ & 109.3 & $\mathrm{O} 54-\mathrm{C} 57-\mathrm{H} 57 \mathrm{~A}$ & 109.5 \\
\hline $\mathrm{N} 4-\mathrm{C} 5-\mathrm{H} 5 \mathrm{~B}$ & 109.3 & $\mathrm{O} 54-\mathrm{C} 57-\mathrm{H} 57 \mathrm{~B}$ & 109.5 \\
\hline $\mathrm{C} 6-\mathrm{C} 5-\mathrm{H} 5 \mathrm{~B}$ & 109.3 & $\mathrm{H} 57 \mathrm{~A}-\mathrm{C} 57-\mathrm{H} 57 \mathrm{~B}$ & 109.5 \\
\hline $\mathrm{H} 5 \mathrm{~A}-\mathrm{C} 5-\mathrm{H} 5 \mathrm{~B}$ & 108.0 & O54-C57-H57C & 109.5 \\
\hline $\mathrm{N} 1-\mathrm{C} 6-\mathrm{C} 5$ & $110.23(17)$ & $\mathrm{H} 57 \mathrm{~A}-\mathrm{C} 57-\mathrm{H} 57 \mathrm{C}$ & 109.5 \\
\hline $\mathrm{N} 1-\mathrm{C} 6-\mathrm{H} 6 \mathrm{~A}$ & 109.6 & $\mathrm{H} 57 \mathrm{~B}-\mathrm{C} 57-\mathrm{H} 57 \mathrm{C}$ & 109.5 \\
\hline $\mathrm{C} 5-\mathrm{C} 6-\mathrm{H} 6 \mathrm{~A}$ & 109.6 & $\mathrm{C} 36-\mathrm{C} 31-\mathrm{C} 32$ & $117.9(2)$ \\
\hline $\mathrm{N} 1-\mathrm{C} 6-\mathrm{H} 6 \mathrm{~B}$ & 109.6 & $\mathrm{C} 36-\mathrm{C} 31-\mathrm{C} 37$ & $121.4(2)$ \\
\hline $\mathrm{C} 5-\mathrm{C} 6-\mathrm{H} 6 \mathrm{~B}$ & 109.6 & $\mathrm{C} 32-\mathrm{C} 31-\mathrm{C} 37$ & $120.8(2)$ \\
\hline $\mathrm{H} 6 \mathrm{~A}-\mathrm{C} 6-\mathrm{H} 6 \mathrm{~B}$ & 108.1 & $\mathrm{C} 33-\mathrm{C} 32-\mathrm{C} 31$ & $120.4(2)$ \\
\hline $\mathrm{C} 26-\mathrm{C} 21-\mathrm{C} 22$ & $116.0(4)$ & $\mathrm{C} 33-\mathrm{C} 32-\mathrm{H} 32$ & 119.8 \\
\hline $\mathrm{C} 26-\mathrm{C} 21-\mathrm{N} 4$ & $119.5(8)$ & $\mathrm{C} 31-\mathrm{C} 32-\mathrm{H} 32$ & 119.8 \\
\hline $\mathrm{C} 22-\mathrm{C} 21-\mathrm{N} 4$ & $124.4(8)$ & $\mathrm{C} 34-\mathrm{C} 33-\mathrm{C} 32$ & $120.6(3)$ \\
\hline $\mathrm{C} 23-\mathrm{C} 22-\mathrm{C} 21$ & $121.2(4)$ & $\mathrm{C} 34-\mathrm{C} 33-\mathrm{H} 33$ & 119.7 \\
\hline $\mathrm{C} 23-\mathrm{C} 22-\mathrm{H} 22$ & 119.4 & $\mathrm{C} 32-\mathrm{C} 33-\mathrm{H} 33$ & 119.7 \\
\hline $\mathrm{C} 21-\mathrm{C} 22-\mathrm{H} 22$ & 119.4 & $\mathrm{C} 35-\mathrm{C} 34-\mathrm{C} 33$ & $119.8(3)$ \\
\hline $\mathrm{C} 24-\mathrm{C} 23-\mathrm{C} 22$ & $121.5(4)$ & $\mathrm{C} 35-\mathrm{C} 34-\mathrm{H} 34$ & 120.1 \\
\hline
\end{tabular}


$\mathrm{C} 24-\mathrm{C} 23-\mathrm{H} 23$
$\mathrm{C} 22-\mathrm{C} 23-\mathrm{H} 23$
$\mathrm{C} 23-\mathrm{C} 24-\mathrm{C} 25$
$\mathrm{C} 23-\mathrm{C} 24-\mathrm{O} 24$
$\mathrm{C} 25-\mathrm{C} 24-\mathrm{O} 24$
$\mathrm{C} 24-\mathrm{C} 25-\mathrm{C} 26$
$\mathrm{C} 24-\mathrm{C} 25-\mathrm{H} 25$
$\mathrm{C} 26-\mathrm{C} 25-\mathrm{H} 25$
$\mathrm{C} 21-\mathrm{C} 26-\mathrm{C} 25$
$\mathrm{C} 21-\mathrm{C} 26-\mathrm{H} 26$
$\mathrm{C} 25-\mathrm{C} 26-\mathrm{H} 26$
$\mathrm{C} 24-\mathrm{O} 24-\mathrm{C} 27$

$\mathrm{C} 6-\mathrm{N} 1-\mathrm{C} 2-\mathrm{C} 3$

$\mathrm{N} 1-\mathrm{C} 2-\mathrm{C} 3-\mathrm{N} 4$

$\mathrm{C} 2-\mathrm{C} 3-\mathrm{N} 4-\mathrm{C} 51$

$\mathrm{C} 2-\mathrm{C} 3-\mathrm{N} 4-\mathrm{C} 21$

$\mathrm{C} 2-\mathrm{C} 3-\mathrm{N} 4-\mathrm{C} 5$

$\mathrm{C} 51-\mathrm{N} 4-\mathrm{C} 5-\mathrm{C} 6$

$\mathrm{C} 21-\mathrm{N} 4-\mathrm{C} 5-\mathrm{C} 6$

$\mathrm{C} 3-\mathrm{N} 4-\mathrm{C} 5-\mathrm{C} 6$

$\mathrm{C} 2-\mathrm{N} 1-\mathrm{C} 6-\mathrm{C} 5$

N4-C5-C6-N1

$\mathrm{C} 51-\mathrm{N} 4-\mathrm{C} 21-\mathrm{C} 26$

$\mathrm{C} 3-\mathrm{N} 4-\mathrm{C} 21-\mathrm{C} 26$

$\mathrm{C} 5-\mathrm{N} 4-\mathrm{C} 21-\mathrm{C} 26$

$\mathrm{C} 51-\mathrm{N} 4-\mathrm{C} 21-\mathrm{C} 22$

$\mathrm{C} 3-\mathrm{N} 4-\mathrm{C} 21-\mathrm{C} 22$

$\mathrm{C} 5-\mathrm{N} 4-\mathrm{C} 21-\mathrm{C} 22$

$\mathrm{C} 26-\mathrm{C} 21-\mathrm{C} 22-\mathrm{C} 23$

$\mathrm{N} 4-\mathrm{C} 21-\mathrm{C} 22-\mathrm{C} 23$

$\mathrm{C} 21-\mathrm{C} 22-\mathrm{C} 23-\mathrm{C} 24$

$\mathrm{C} 22-\mathrm{C} 23-\mathrm{C} 24-\mathrm{C} 25$

$\mathrm{C} 22-\mathrm{C} 23-\mathrm{C} 24-\mathrm{O} 24$

$\mathrm{C} 23-\mathrm{C} 24-\mathrm{C} 25-\mathrm{C} 26$

$\mathrm{O} 24-\mathrm{C} 24-\mathrm{C} 25-\mathrm{C} 26$

$\mathrm{C} 22-\mathrm{C} 21-\mathrm{C} 26-\mathrm{C} 25$

N4-C21-C26-C25

$\mathrm{C} 24-\mathrm{C} 25-\mathrm{C} 26-\mathrm{C} 21$

$\mathrm{C} 23-\mathrm{C} 24-\mathrm{O} 24-\mathrm{C} 27$

$\mathrm{C} 25-\mathrm{C} 24-\mathrm{O} 24-\mathrm{C} 27$

$\mathrm{C} 21-\mathrm{N} 4-\mathrm{C} 51-\mathrm{C} 56$
119.3

119.3

$118.5(4)$

$116.6(5)$

$124.6(6)$

119.5 (7)

120.3

120.3

122.9 (5)

118.5

118.5

$118.1(6)$

$-57.3(2)$

55.6 (2)

168.3 (19)

$175.8(8)$

$-53.8(2)$

$-164.9(15)$

$-176.0(7)$

54.3 (2)

58.3 (2)

$-57.0(2)$

$-49(10)$

170.7 (16)

42 (2)

$128(12)$

$-12.5(17)$

-141.2 (12)

2 (2)

$-174.5(11)$

$-2.6(14)$

4.6 (17)

$178.6(15)$

$-6(3)$

$-180(2)$

-4 (3)

173 (2)

7 (4)

$171(2)$

$-16(3)$

$123(14)$
$\mathrm{C} 33-\mathrm{C} 34-\mathrm{H} 34$

C34-C35-C36

C $34-\mathrm{C} 35-\mathrm{H} 35$

C $36-\mathrm{C} 35-\mathrm{H} 35$

$\mathrm{C} 35-\mathrm{C} 36-\mathrm{C} 31$

$\mathrm{C} 35-\mathrm{C} 36-\mathrm{H} 36$

$\mathrm{C} 31-\mathrm{C} 36-\mathrm{H} 36$

$\mathrm{O} 32-\mathrm{C} 37-\mathrm{O} 31$

$\mathrm{O} 32-\mathrm{C} 37-\mathrm{C} 31$

$\mathrm{O} 31-\mathrm{C} 37-\mathrm{C} 31$

$\mathrm{H} 41-\mathrm{O} 41-\mathrm{H} 42$

C3-N4-C51-C56

$\mathrm{C} 5-\mathrm{N} 4-\mathrm{C} 51-\mathrm{C} 56$

$\mathrm{C} 21-\mathrm{N} 4-\mathrm{C} 51-\mathrm{C} 52$

C3-N4-C51-C52

$\mathrm{C} 5-\mathrm{N} 4-\mathrm{C} 51-\mathrm{C} 52$

C56-C51-C52-C53

N4-C51-C52-C53

$\mathrm{C} 51-\mathrm{C} 52-\mathrm{C} 53-\mathrm{C} 54$

$\mathrm{C} 52-\mathrm{C} 53-\mathrm{C} 54-\mathrm{C} 55$

$\mathrm{C} 52-\mathrm{C} 53-\mathrm{C} 54-\mathrm{O} 54$

C53-C54-C55-C56

$\mathrm{O} 54-\mathrm{C} 54-\mathrm{C} 55-\mathrm{C} 56$

C52-C51-C56-C55

N4-C51-C56-C55

C54-C55-C56-C51

$\mathrm{C} 53-\mathrm{C} 54-\mathrm{O} 54-\mathrm{C} 57$

$\mathrm{C} 55-\mathrm{C} 54-\mathrm{O} 54-\mathrm{C} 57$

$\mathrm{C} 36-\mathrm{C} 31-\mathrm{C} 32-\mathrm{C} 33$

$\mathrm{C} 37-\mathrm{C} 31-\mathrm{C} 32-\mathrm{C} 33$

$\mathrm{C} 31-\mathrm{C} 32-\mathrm{C} 33-\mathrm{C} 34$

$\mathrm{C} 32-\mathrm{C} 33-\mathrm{C} 34-\mathrm{C} 35$

$\mathrm{C} 33-\mathrm{C} 34-\mathrm{C} 35-\mathrm{C} 36$

$\mathrm{C} 34-\mathrm{C} 35-\mathrm{C} 36-\mathrm{C} 31$

$\mathrm{C} 32-\mathrm{C} 31-\mathrm{C} 36-\mathrm{C} 35$

$\mathrm{C} 37-\mathrm{C} 31-\mathrm{C} 36-\mathrm{C} 35$

$\mathrm{C} 36-\mathrm{C} 31-\mathrm{C} 37-\mathrm{O} 32$

$\mathrm{C} 32-\mathrm{C} 31-\mathrm{C} 37-\mathrm{O} 32$

$\mathrm{C} 36-\mathrm{C} 31-\mathrm{C} 37-\mathrm{O} 31$

$\mathrm{C} 32-\mathrm{C} 31-\mathrm{C} 37-\mathrm{O} 31$
120.1

120.5 (2)

119.7

119.7

120.9 (2)

119.6

119.6

124.1 (3)

117.3 (3)

118.6 (2)

111 (3)

166 (4)

$30(5)$

$-56(9)$

-13 (4)

-149 (2)

-4 (5)

175 (2)

5 (3)

-12 (4)

$-176(3)$

18 (6)

$-180(5)$

10 (7)

$-169(4)$

-17 (8)

161 (4)

$-2(7)$

0.7 (3)

-179.3 (2)

0.3 (4)

-1.0 (4)

0.7 (4)

0.4 (3)

-1.0 (3)

178.9 (2)

175.1 (2)

$-5.0(3)$

$-3.1(3)$

176.9 (2)

Hydrogen-bond geometry $\left(\AA,{ }^{\circ}\right)$

\begin{tabular}{lllll}
\hline$D-\mathrm{H} \cdots A$ & $D-\mathrm{H}$ & $\mathrm{H} \cdots A$ & $D \cdots A$ & $D-\mathrm{H} \cdots A$ \\
\hline $\mathrm{N} 1-\mathrm{H} 11 \cdots \mathrm{O} 31$ & $0.90(2)$ & $1.88(2)$ & $2.777(3)$ & $174.1(19)$ \\
$\mathrm{N} 1-\mathrm{H} 12 \cdots \mathrm{O} 41$ & $0.97(2)$ & $1.85(2)$ & $2.808(3)$ & $169.7(18)$
\end{tabular}




$\begin{array}{lllll}\mathrm{O} 41-\mathrm{H} 41 \cdots \mathrm{O} 32^{\mathrm{i}} & 0.88(3) & 1.75(3) & 2.631(3) & 177(3) \\ \mathrm{O} 41-\mathrm{H} 42 \cdots \mathrm{O} 31^{\mathrm{ii}} & 0.91(3) & 1.87(3) & 2.763(3) & 169(3) \\ \mathrm{C} 2-\mathrm{H} 2 B \cdots \mathrm{O} 31^{\mathrm{iii}} & 0.97 & 2.54 & 3.485(3) & 165 \\ \mathrm{C} 22-\mathrm{H} 22 \cdots C g 1^{\mathrm{ii}} & 0.93 & 2.85 & 3.603(3) & 139 \\ \mathrm{C} 26-\mathrm{H} 26 \cdots C g 1^{\mathrm{iv}} & 0.93 & 2.90 & 3.62(2) & 135 \\ \mathrm{C} 56-\mathrm{H} 56 \cdots C g 1^{\mathrm{iv}} & 0.93 & 2.64 & 3.41(5) & 141\end{array}$

Symmetry codes: (i) $-x+1,-y+1,-z+1$; (ii) $-x+2,-y+1,-z+1$; (iii) $-x+2,-y+2,-z+1$; (iv) $-x+1,-y+2,-z+1$.

4-(4-Methoxyphenyl) piperazin-1-ium 4-fluorobenzoate monohydrate (II)

Crystal data

$\mathrm{C}_{11} \mathrm{H}_{17} \mathrm{~N}_{2} \mathrm{O}^{+} \cdot \mathrm{C}_{7} \mathrm{H}_{4} \mathrm{FO}_{2}^{-} \cdot \mathrm{H}_{2} \mathrm{O}$

$M_{r}=350.38$

Triclinic, $P \overline{1}$

$a=6.256(1) \AA$

$b=7.489(1) \AA$

$c=19.097(2) \AA$

$\alpha=84.19(1)^{\circ}$

$\beta=86.98(2)^{\circ}$

$\gamma=84.62(2)^{\circ}$

$V=885.4(2) \AA^{3}$

Data collection

Oxford Diffraction Xcalibur with Sapphire CCD diffractometer

Radiation source: Enhance (Mo) X-ray Source

Graphite monochromator

$\omega$ scans

Absorption correction: multi-scan

(CrysAlis RED; Oxford Diffraction, 2009)

$T_{\min }=0.973, T_{\max }=0.996$

\section{Refinement}

Refinement on $F^{2}$

Least-squares matrix: full

$R\left[F^{2}>2 \sigma\left(F^{2}\right)\right]=0.066$

$w R\left(F^{2}\right)=0.128$

$S=1.01$

3477 reflections

265 parameters

17 restraints
$Z=2$

$F(000)=372$

$D_{\mathrm{x}}=1.314 \mathrm{Mg} \mathrm{m}^{-3}$

Mo $K \alpha$ radiation, $\lambda=0.71073 \AA$

Cell parameters from 3771 reflections

$\theta=2.9-27.9^{\circ}$

$\mu=0.10 \mathrm{~mm}^{-1}$

$T=293 \mathrm{~K}$

Plate, colourless

$0.40 \times 0.24 \times 0.04 \mathrm{~mm}$

5760 measured reflections

3477 independent reflections

1355 reflections with $I>2 \sigma(I)$

$R_{\text {int }}=0.046$

$\theta_{\max }=26.1^{\circ}, \theta_{\min }=2.9^{\circ}$

$h=-7 \rightarrow 6$

$k=-9 \rightarrow 9$

$l=-23 \rightarrow 22$
Primary atom site location: difference Fourier map

Hydrogen site location: mixed

$\mathrm{H}$ atoms treated by a mixture of independent and constrained refinement

$w=1 /\left[\sigma^{2}\left(F_{\mathrm{o}}^{2}\right)+(0.0404 P)^{2}\right]$ where $P=\left(F_{\mathrm{o}}^{2}+2 F_{\mathrm{c}}{ }^{2}\right) / 3$

$(\Delta / \sigma)_{\max }<0.001$

$\Delta \rho_{\max }=0.13$ e $\AA^{-3}$

$\Delta \rho_{\min }=-0.14$ e $\AA^{-3}$

Special details

Experimental. Compound (II). IR ( $\left.\mathrm{KBr}, \mathrm{cm}^{-1}\right) 3317(\mathrm{OH}), 3011\left(\mathrm{NH}_{2}\right), 2838\left(\mathrm{OCH}_{3}\right), 1588(\mathrm{COO}), 1365(\mathrm{CF}) \mathrm{NMR}$ $\left(\mathrm{CDCl}_{3}\right) \delta\left({ }^{1} \mathrm{H}\right) 3.23\left(\mathrm{~m}, 4 \mathrm{H}\right.$, piperazine), $3.29(\mathrm{~m}, 4 \mathrm{H}$, piperazine $), 3.77\left(\mathrm{~s}, 3 \mathrm{H}, \mathrm{OCH}_{3}\right), 6.86(\mathrm{~m}, 4 \mathrm{H}$, methoxyphenyl), 7.05 (m, 2H, fluorophenyl), 8.05 ( $\mathrm{m}, 2 \mathrm{H}$, fluorophenyl).

Geometry. All esds (except the esd in the dihedral angle between two 1.s. planes) are estimated using the full covariance matrix. The cell esds are taken into account individually in the estimation of esds in distances, angles and torsion angles; correlations between esds in cell parameters are only used when they are defined by crystal symmetry. An approximate (isotropic) treatment of cell esds is used for estimating esds involving 1.s. planes. 
Fractional atomic coordinates and isotropic or equivalent isotropic displacement parameters $\left(\hat{A}^{2}\right)$

\begin{tabular}{|c|c|c|c|c|c|}
\hline & $x$ & $y$ & $z$ & $U_{\text {iso }} * / U_{\text {eq }}$ & Occ. $(<1)$ \\
\hline N1 & $0.7813(5)$ & $0.7442(3)$ & $0.45140(15)$ & $0.0536(8)$ & \\
\hline H11 & $0.823(4)$ & $0.778(3)$ & $0.5031(15)$ & $0.064 *$ & \\
\hline H12 & $0.749(4)$ & $0.634(4)$ & 0.4565 (14) & $0.064 *$ & \\
\hline $\mathrm{C} 2$ & $0.9661(5)$ & $0.7540(4)$ & $0.39966(16)$ & $0.0599(9)$ & \\
\hline $\mathrm{H} 2 \mathrm{~A}$ & 1.0848 & 0.6706 & 0.4164 & $0.072 *$ & \\
\hline $\mathrm{H} 2 \mathrm{~B}$ & 1.0131 & 0.8746 & 0.3952 & $0.072 *$ & \\
\hline $\mathrm{C} 3$ & $0.9056(5)$ & $0.7083(4)$ & $0.32889(15)$ & $0.0574(9)$ & \\
\hline $\mathrm{H} 3 \mathrm{~A}$ & 1.0264 & 0.7229 & 0.2955 & $0.069^{*}$ & \\
\hline H3B & 0.8755 & 0.5829 & 0.3326 & $0.069^{*}$ & \\
\hline N4 & $0.7185(4)$ & $0.8206(3)$ & $0.30227(12)$ & $0.0444(6)$ & \\
\hline C5 & 0.5377 & $0.8151(4)$ & $0.35391(14)$ & $0.0527(8)$ & \\
\hline $\mathrm{H} 5 \mathrm{~A}$ & 0.4900 & 0.6947 & 0.3596 & $0.063 *$ & \\
\hline $\mathrm{H} 5 \mathrm{~B}$ & 0.4192 & 0.8977 & 0.3365 & $0.063^{*}$ & \\
\hline C6 & $0.5960(5)$ & $0.8649(4)$ & $0.42410(14)$ & $0.0567(9)$ & \\
\hline H6A & 0.6324 & 0.9887 & 0.4194 & $0.068 *$ & \\
\hline H6B & 0.4736 & 0.8556 & 0.4572 & $0.068^{*}$ & \\
\hline $\mathrm{C} 21$ & $0.670(3)$ & $0.795(6)$ & $0.2319(6)$ & $0.0442(18)$ & $0.81(3)$ \\
\hline $\mathrm{C} 22$ & $0.8062(12)$ & $0.6932(18)$ & $0.1883(4)$ & $0.064(2)$ & $0.81(3)$ \\
\hline $\mathrm{H} 22$ & 0.9351 & 0.6378 & 0.2053 & $0.077^{*}$ & $0.81(3)$ \\
\hline $\mathrm{C} 23$ & $0.7544(9)$ & $0.673(2)$ & $0.1207(3)$ & $0.069(3)$ & $0.81(3)$ \\
\hline $\mathrm{H} 23$ & 0.8520 & 0.6083 & 0.0923 & $0.083^{*}$ & $0.81(3)$ \\
\hline $\mathrm{C} 24$ & $0.5644(10)$ & $0.7455(18)$ & $0.0942(3)$ & $0.058(2)$ & $0.81(3)$ \\
\hline $\mathrm{C} 25$ & $0.4255(15)$ & $0.846(3)$ & $0.1357(5)$ & $0.0680(18)$ & $0.81(3)$ \\
\hline $\mathrm{H} 25$ & 0.2967 & 0.9002 & 0.1182 & $0.082 *$ & $0.81(3)$ \\
\hline $\mathrm{C} 26$ & $0.4782(18)$ & $0.866(3)$ & $0.2037(5)$ & $0.062(2)$ & $0.81(3)$ \\
\hline $\mathrm{H} 26$ & 0.3798 & 0.9313 & 0.2317 & $0.074 *$ & $0.81(3)$ \\
\hline $\mathrm{O} 24$ & $0.5299(13)$ & 0.7159 (19) & 0.0261 & $0.095(2)$ & $0.81(3)$ \\
\hline $\mathrm{C} 27$ & $0.3282(18)$ & $0.772(3)$ & $-0.0015(6)$ & $0.107(4)$ & $0.81(3)$ \\
\hline $\mathrm{H} 27 \mathrm{~A}$ & 0.2181 & 0.7178 & 0.0279 & $0.160 *$ & $0.81(3)$ \\
\hline H27B & 0.3249 & 0.7366 & -0.0483 & $0.160 *$ & $0.81(3)$ \\
\hline $\mathrm{H} 27 \mathrm{C}$ & 0.3035 & 0.9011 & -0.0029 & $0.160 *$ & $0.81(3)$ \\
\hline C51 & $0.655(11)$ & $0.79(3)$ & $0.236(2)$ & $0.0442(18)$ & $0.19(3)$ \\
\hline $\mathrm{C} 52$ & $0.810(6)$ & $0.754(6)$ & 0.1833 (17) & $0.064(2)$ & $0.19(3)$ \\
\hline H52 & 0.9531 & 0.7290 & 0.1949 & $0.077^{*}$ & $0.19(3)$ \\
\hline C53 & $0.756(4)$ & $0.752(6)$ & $0.1146(15)$ & $0.069(3)$ & $0.19(3)$ \\
\hline H53 & 0.8593 & 0.7106 & 0.0818 & $0.083^{*}$ & $0.19(3)$ \\
\hline $\mathrm{C} 54$ & $0.554(4)$ & $0.808(5)$ & $0.0936(13)$ & $0.058(2)$ & $0.19(3)$ \\
\hline $\mathrm{C} 55$ & $0.396(6)$ & $0.842(13)$ & $0.144(2)$ & $0.0680(18)$ & $0.19(3)$ \\
\hline H55 & 0.2524 & 0.8581 & 0.1327 & $0.082 *$ & $0.19(3)$ \\
\hline $\mathrm{C} 56$ & $0.453(7)$ & $0.851(13)$ & $0.213(2)$ & $0.062(2)$ & $0.19(3)$ \\
\hline H56 & 0.3509 & 0.8987 & 0.2444 & $0.074 *$ & $0.19(3)$ \\
\hline O54 & $0.512(5)$ & $0.786(6)$ & $0.0251(14)$ & $0.095(2)$ & $0.19(3)$ \\
\hline $\mathrm{C} 57$ & $0.300(7)$ & $0.827(12)$ & $0.004(3)$ & $0.107(4)$ & $0.19(3)$ \\
\hline H57A & 0.2472 & 0.9444 & 0.0166 & $0.160 *$ & $0.19(3)$ \\
\hline H57B & 0.2101 & 0.7391 & 0.0266 & $0.160 *$ & $0.19(3)$ \\
\hline
\end{tabular}




$\begin{array}{lllll}\text { H57C } & 0.2965 & 0.8256 & -0.0463 & 0.160^{*} \\ \text { C31 } & 0.8132(5) & 0.7284(4) & 0.70495(17) & 0.0485(8) \\ \text { C32 } & 0.6742(5) & 0.6533(4) & 0.7564(2) & 0.0676(10) \\ \text { H32 } & 0.5542 & 0.6028 & 0.7431 & 0.081^{*} \\ \text { C33 } & 0.7114(6) & 0.6527(5) & 0.8267(2) & 0.0798(11) \\ \text { H33 } & 0.6181 & 0.6022 & 0.8611 & 0.096^{*} \\ \text { C34 } & 0.8883(7) & 0.7278(5) & 0.8448(2) & 0.0752(11) \\ \text { F34 } & 0.9236(3) & 0.7291(3) & 0.91437(11) & 0.1208(9) \\ \text { C35 } & 1.0294(5) & 0.8010(4) & 0.7961(2) & 0.0658(10) \\ \text { H35 } & 1.1495 & 0.8505 & 0.8100 & 0.079^{*} \\ \text { C36 } & 0.9911(5) & 0.8003(4) & 0.72606(17) & 0.0542(9) \\ \text { H36 } & 1.0870 & 0.8494 & 0.6922 & 0.065^{*} \\ \text { C37 } & 0.7690(6) & 0.7297(5) & 0.6289(2) & 0.0614(10) \\ \text { O31 } & 0.8943(4) & 0.8069(3) & 0.58380(12) & 0.0715(7) \\ \text { O32 } & 0.6112(4) & 0.6566(4) & 0.61332(13) & 0.1091(10) \\ \text { O41 } & 0.7239(4) & 0.3737(3) & 0.46049(12) & 0.0700(8) \\ \text { H41 } & 0.615(6) & 0.356(5) & 0.4356(17) & 0.105^{*} \\ \text { H42 } & 0.835(6) & 0.312(5) & 0.4379(18) & 0.105^{*}\end{array}$

Atomic displacement parameters $\left(\AA^{2}\right)$

\begin{tabular}{|c|c|c|c|c|c|c|}
\hline & $U^{11}$ & $U^{22}$ & $U^{33}$ & $U^{12}$ & $U^{13}$ & $U^{23}$ \\
\hline N1 & $0.065(2)$ & $0.0429(18)$ & $0.0550(18)$ & $-0.0089(16)$ & $-0.0145(16)$ & $-0.0061(15)$ \\
\hline $\mathrm{C} 2$ & $0.050(2)$ & $0.060(3)$ & $0.070(2)$ & $-0.0047(18)$ & -0.0107 (19) & $-0.0069(18)$ \\
\hline $\mathrm{C} 3$ & $0.049(2)$ & $0.065(2)$ & $0.059(2)$ & $-0.0019(18)$ & $-0.0035(17)$ & $-0.0091(18)$ \\
\hline N4 & $0.0405(16)$ & $0.0437(17)$ & $0.0483(16)$ & $0.0016(13)$ & $-0.0044(13)$ & $-0.0054(12)$ \\
\hline $\mathrm{C} 5$ & $0.049(2)$ & $0.057(2)$ & $0.052(2)$ & $-0.0005(16)$ & $-0.0015(17)$ & $-0.0083(16)$ \\
\hline C6 & $0.059(2)$ & $0.057(2)$ & $0.053(2)$ & $0.0029(18)$ & $-0.0011(17)$ & $-0.0049(17)$ \\
\hline $\mathrm{C} 21$ & $0.044(3)$ & $0.042(3)$ & $0.047(2)$ & $-0.004(4)$ & $0.001(2)$ & $-0.008(3)$ \\
\hline $\mathrm{C} 22$ & $0.055(2)$ & $0.070(7)$ & $0.068(3)$ & $0.009(3)$ & $-0.005(2)$ & $-0.020(3)$ \\
\hline $\mathrm{C} 23$ & $0.063(3)$ & $0.080(8)$ & $0.067(3)$ & $0.009(3)$ & $0.004(2)$ & $-0.030(4)$ \\
\hline $\mathrm{C} 24$ & $0.066(3)$ & $0.058(6)$ & $0.053(3)$ & $-0.007(3)$ & $-0.003(2)$ & $-0.017(3)$ \\
\hline $\mathrm{C} 25$ & $0.061(3)$ & $0.087(3)$ & $0.054(4)$ & $0.012(4)$ & -0.009 (3) & -0.009 (4) \\
\hline $\mathrm{C} 26$ & $0.056(3)$ & $0.070(5)$ & $0.056(3)$ & $0.016(4)$ & $-0.001(2)$ & $-0.013(4)$ \\
\hline $\mathrm{O} 24$ & $0.094(3)$ & $0.129(7)$ & 0.0660 (19) & 0.008 (4) & $-0.0132(16)$ & -0.039 (3) \\
\hline $\mathrm{C} 27$ & $0.102(5)$ & $0.156(14)$ & 0.067 (4) & $-0.004(5)$ & $-0.027(4)$ & $-0.023(4)$ \\
\hline $\mathrm{C} 51$ & $0.044(3)$ & $0.042(3)$ & $0.047(2)$ & $-0.004(4)$ & $0.001(2)$ & $-0.008(3)$ \\
\hline $\mathrm{C} 52$ & $0.055(2)$ & $0.070(7)$ & $0.068(3)$ & 0.009 (3) & $-0.005(2)$ & -0.020 \\
\hline $\mathrm{C} 53$ & $0.063(3)$ & $0.080(8)$ & $0.067(3)$ & $0.009(3)$ & $0.004(2)$ & $-0.030(4)$ \\
\hline $\mathrm{C} 54$ & $0.066(3)$ & $0.058(6)$ & $0.053(3)$ & $-0.007(3)$ & $-0.003(2)$ & $-0.017(3)$ \\
\hline C55 & $0.061(3)$ & $0.087(3)$ & $0.054(4)$ & $0.012(4)$ & -0.009 & -0.009 \\
\hline $\mathrm{C} 56$ & $0.056(3)$ & $0.070(5)$ & $0.056(3)$ & $0.016(4)$ & $-0.001(2)$ & $-0.013(4)$ \\
\hline O54 & $0.094(3)$ & $0.129(7)$ & 0.0660 (19) & $0.008(4)$ & $-0.0132(16)$ & -0.039 (3) \\
\hline $\mathrm{C} 57$ & $0.102(5)$ & $0.156(14)$ & 0.067 (4) & $-0.004(5)$ & $-0.027(4)$ & $-0.023(4)$ \\
\hline $\mathrm{C} 31$ & $0.045(2)$ & $0.039(2)$ & $0.063(2)$ & $0.0008(16)$ & $-0.0084(18)$ & $-0.0119(16)$ \\
\hline $\mathrm{C} 32$ & $0.051(2)$ & $0.055(3)$ & $0.099(3)$ & $-0.0062(18)$ & $-0.002(2)$ & $-0.017(2)$ \\
\hline C33 & $0.072(3)$ & $0.073(3)$ & $0.089(3)$ & $-0.004(2)$ & $0.020(2)$ & $-0.001(2)$ \\
\hline C34 & $0.083(3)$ & $0.084(3)$ & $0.057(3)$ & $0.003(2)$ & -0.009 (2) & $-0.004(2)$ \\
\hline
\end{tabular}




\begin{tabular}{lllllll} 
F34 & $0.131(2)$ & $0.164(2)$ & $0.0633(15)$ & $0.0008(17)$ & $-0.0068(13)$ & $-0.0036(14)$ \\
C35 & $0.059(2)$ & $0.068(3)$ & $0.072(3)$ & $-0.0106(19)$ & $-0.011(2)$ & $-0.006(2)$ \\
C36 & $0.056(2)$ & $0.052(2)$ & $0.056(2)$ & $-0.0059(18)$ & $-0.0122(17)$ & $-0.0070(16)$ \\
C37 & $0.057(3)$ & $0.042(2)$ & $0.087(3)$ & $0.0070(19)$ & $-0.022(2)$ & $-0.019(2)$ \\
O31 & $0.0848(18)$ & $0.0686(18)$ & $0.0638(17)$ & $-0.0057(15)$ & $-0.0208(14)$ & $-0.0123(13)$ \\
O32 & $0.0871(19)$ & $0.129(3)$ & $0.124(2)$ & $-0.0362(18)$ & $-0.0427(16)$ & $-0.0343(17)$ \\
O41 & $0.0693(17)$ & $0.0628(18)$ & $0.0816(18)$ & $-0.0137(14)$ & $-0.0227(13)$ & $-0.0073(13)$ \\
\hline
\end{tabular}

Geometric parameters $\left(\AA,{ }^{\circ}\right)$

\begin{tabular}{|c|c|c|c|}
\hline $\mathrm{N} 1-\mathrm{C} 2$ & $1.483(4)$ & $\mathrm{C} 27-\mathrm{H} 27 \mathrm{C}$ & 0.9600 \\
\hline $\mathrm{N} 1-\mathrm{C} 6$ & $1.485(4)$ & $\mathrm{C} 51-\mathrm{C} 56$ & $1.380(9)$ \\
\hline $\mathrm{N} 1-\mathrm{H} 11$ & $1.09(3)$ & $\mathrm{C} 51-\mathrm{C} 52$ & $1.390(19)$ \\
\hline $\mathrm{N} 1-\mathrm{H} 12$ & $0.87(3)$ & $\mathrm{C} 52-\mathrm{C} 53$ & $1.374(9)$ \\
\hline $\mathrm{C} 2-\mathrm{C} 3$ & $1.503(3)$ & C52-H52 & 0.9300 \\
\hline $\mathrm{C} 2-\mathrm{H} 2 \mathrm{~A}$ & 0.9700 & $\mathrm{C} 53-\mathrm{C} 54$ & $1.362(9)$ \\
\hline $\mathrm{C} 2-\mathrm{H} 2 \mathrm{~B}$ & 0.9700 & $\mathrm{C} 53-\mathrm{H} 53$ & 0.9300 \\
\hline $\mathrm{C} 3-\mathrm{N} 4$ & $1.458(3)$ & C54-C55 & $1.370(13)$ \\
\hline $\mathrm{C} 3-\mathrm{H} 3 \mathrm{~A}$ & 0.9700 & $\mathrm{C} 54-\mathrm{O} 54$ & $1.377(9)$ \\
\hline $\mathrm{C} 3-\mathrm{H} 3 \mathrm{~B}$ & 0.9700 & $\mathrm{C} 55-\mathrm{C} 56$ & $1.383(9)$ \\
\hline $\mathrm{N} 4-\mathrm{C} 51$ & $1.39(5)$ & C55-H55 & 0.9300 \\
\hline $\mathrm{N} 4-\mathrm{C} 21$ & $1.429(10)$ & C56-H56 & 0.9300 \\
\hline $\mathrm{N} 4-\mathrm{C} 5$ & $1.462(3)$ & $\mathrm{O} 54-\mathrm{C} 57$ & $1.408(10)$ \\
\hline $\mathrm{C} 5-\mathrm{C} 6$ & $1.499(3)$ & C57-H57A & 0.9600 \\
\hline $\mathrm{C} 5-\mathrm{H} 5 \mathrm{~A}$ & 0.9700 & C57-H57B & 0.9600 \\
\hline C5-H5B & 0.9700 & $\mathrm{C} 57-\mathrm{H} 57 \mathrm{C}$ & 0.9600 \\
\hline C6-H6A & 0.9700 & $\mathrm{C} 31-\mathrm{C} 36$ & $1.377(3)$ \\
\hline C6-H6B & 0.9700 & $\mathrm{C} 31-\mathrm{C} 32$ & $1.388(4)$ \\
\hline $\mathrm{C} 21-\mathrm{C} 26$ & $1.379(6)$ & $\mathrm{C} 31-\mathrm{C} 37$ & $1.491(4)$ \\
\hline $\mathrm{C} 21-\mathrm{C} 22$ & $1.389(15)$ & $\mathrm{C} 32-\mathrm{C} 33$ & $1.374(4)$ \\
\hline $\mathrm{C} 22-\mathrm{C} 23$ & $1.374(4)$ & C $32-\mathrm{H} 32$ & 0.9300 \\
\hline $\mathrm{C} 22-\mathrm{H} 22$ & 0.9300 & $\mathrm{C} 33-\mathrm{C} 34$ & $1.363(4)$ \\
\hline $\mathrm{C} 23-\mathrm{C} 24$ & $1.361(5)$ & C $33-\mathrm{H} 33$ & 0.9300 \\
\hline $\mathrm{C} 23-\mathrm{H} 23$ & 0.9300 & $\mathrm{C} 34-\mathrm{C} 35$ & $1.358(4)$ \\
\hline $\mathrm{C} 24-\mathrm{C} 25$ & $1.369(9)$ & C34-F34 & $1.360(4)$ \\
\hline $\mathrm{C} 24-\mathrm{O} 24$ & $1.373(4)$ & $\mathrm{C} 35-\mathrm{C} 36$ & $1.373(4)$ \\
\hline $\mathrm{C} 25-\mathrm{C} 26$ & $1.383(5)$ & C $35-\mathrm{H} 35$ & 0.9300 \\
\hline $\mathrm{C} 25-\mathrm{H} 25$ & 0.9300 & C36- & 0.9300 \\
\hline $\mathrm{C} 26-\mathrm{H} 26$ & 0.9300 & $\mathrm{C} 37-\mathrm{O} 32$ & $1.236(3)$ \\
\hline $\mathrm{O} 24-\mathrm{C} 27$ & $1.405(4)$ & $\mathrm{C} 37-\mathrm{O} 31$ & $1.266(4)$ \\
\hline $\mathrm{C} 27-\mathrm{H} 27 \mathrm{~A}$ & 0.9600 & $\mathrm{O} 41-\mathrm{H} 41$ & $0.88(3)$ \\
\hline C27-H27B & 0.9600 & $\mathrm{O} 41-\mathrm{H} 42$ & $0.91(4)$ \\
\hline $\mathrm{C} 2-\mathrm{N} 1-\mathrm{C} 6$ & $109.5(2)$ & $\mathrm{O} 24-\mathrm{C} 27-\mathrm{H} 27 \mathrm{~A}$ & 109.5 \\
\hline $\mathrm{C} 2-\mathrm{N} 1-\mathrm{H} 11$ & $111.6(13)$ & $\mathrm{O} 24-\mathrm{C} 27-\mathrm{H} 27 \mathrm{~B}$ & 109.5 \\
\hline $\mathrm{C} 6-\mathrm{N} 1-\mathrm{H} 11$ & $111.1(13)$ & $\mathrm{H} 27 \mathrm{~A}-\mathrm{C} 27-\mathrm{H} 27 \mathrm{~B}$ & 109.5 \\
\hline $\mathrm{C} 2-\mathrm{N} 1-\mathrm{H} 12$ & $107(2)$ & $\mathrm{O} 24-\mathrm{C} 27-\mathrm{H} 27 \mathrm{C}$ & 109.5 \\
\hline $\mathrm{C} 6-\mathrm{N} 1-\mathrm{H} 12$ & $110.0(19)$ & $\mathrm{H} 27 \mathrm{~A}-\mathrm{C} 27-\mathrm{H} 27 \mathrm{C}$ & 109.5 \\
\hline
\end{tabular}




\begin{tabular}{|c|c|c|c|}
\hline $\mathrm{H} 11-\mathrm{N} 1-\mathrm{H} 12$ & $107(2)$ & $\mathrm{H} 27 \mathrm{~B}-\mathrm{C} 27-\mathrm{H} 27 \mathrm{C}$ & 109.5 \\
\hline $\mathrm{N} 1-\mathrm{C} 2-\mathrm{C} 3$ & $110.9(2)$ & $\mathrm{C} 56-\mathrm{C} 51-\mathrm{C} 52$ & $115.3(18)$ \\
\hline $\mathrm{N} 1-\mathrm{C} 2-\mathrm{H} 2 \mathrm{~A}$ & 109.5 & $\mathrm{C} 56-\mathrm{C} 51-\mathrm{N} 4$ & $122(4)$ \\
\hline $\mathrm{C} 3-\mathrm{C} 2-\mathrm{H} 2 \mathrm{~A}$ & 109.5 & $\mathrm{C} 52-\mathrm{C} 51-\mathrm{N} 4$ & $120(5)$ \\
\hline $\mathrm{N} 1-\mathrm{C} 2-\mathrm{H} 2 \mathrm{~B}$ & 109.5 & $\mathrm{C} 53-\mathrm{C} 52-\mathrm{C} 51$ & $121.2(14)$ \\
\hline $\mathrm{C} 3-\mathrm{C} 2-\mathrm{H} 2 \mathrm{~B}$ & 109.5 & $\mathrm{C} 53-\mathrm{C} 52-\mathrm{H} 52$ & 119.4 \\
\hline $\mathrm{H} 2 \mathrm{~A}-\mathrm{C} 2-\mathrm{H} 2 \mathrm{~B}$ & 108.1 & $\mathrm{C} 51-\mathrm{C} 52-\mathrm{H} 52$ & 119.4 \\
\hline $\mathrm{N} 4-\mathrm{C} 3-\mathrm{C} 2$ & $112.7(2)$ & $\mathrm{C} 54-\mathrm{C} 53-\mathrm{C} 52$ & $121.5(11)$ \\
\hline $\mathrm{N} 4-\mathrm{C} 3-\mathrm{H} 3 \mathrm{~A}$ & 109.0 & $\mathrm{C} 54-\mathrm{C} 53-\mathrm{H} 53$ & 119.3 \\
\hline $\mathrm{C} 2-\mathrm{C} 3-\mathrm{H} 3 \mathrm{~A}$ & 109.0 & $\mathrm{C} 52-\mathrm{C} 53-\mathrm{H} 53$ & 119.3 \\
\hline $\mathrm{N} 4-\mathrm{C} 3-\mathrm{H} 3 \mathrm{~B}$ & 109.0 & $\mathrm{C} 53-\mathrm{C} 54-\mathrm{C} 55$ & $118.3(12)$ \\
\hline $\mathrm{C} 2-\mathrm{C} 3-\mathrm{H} 3 \mathrm{~B}$ & 109.0 & $\mathrm{C} 53-\mathrm{C} 54-\mathrm{O} 54$ & $116.6(13)$ \\
\hline $\mathrm{H} 3 \mathrm{~A}-\mathrm{C} 3-\mathrm{H} 3 \mathrm{~B}$ & 107.8 & $\mathrm{C} 55-\mathrm{C} 54-\mathrm{O} 54$ & $123.2(15)$ \\
\hline $\mathrm{C} 51-\mathrm{N} 4-\mathrm{C} 3$ & $117(4)$ & $\mathrm{C} 54-\mathrm{C} 55-\mathrm{C} 56$ & $119.0(16)$ \\
\hline $\mathrm{C} 21-\mathrm{N} 4-\mathrm{C} 3$ & $114.4(9)$ & $\mathrm{C} 54-\mathrm{C} 55-\mathrm{H} 55$ & 120.5 \\
\hline $\mathrm{C} 51-\mathrm{N} 4-\mathrm{C} 5$ & $111(4)$ & $\mathrm{C} 56-\mathrm{C} 55-\mathrm{H} 55$ & 120.5 \\
\hline $\mathrm{C} 21-\mathrm{N} 4-\mathrm{C} 5$ & $115.5(10)$ & $\mathrm{C} 51-\mathrm{C} 56-\mathrm{C} 55$ & $122.8(13)$ \\
\hline $\mathrm{C} 3-\mathrm{N} 4-\mathrm{C} 5$ & $110.9(2)$ & $\mathrm{C} 51-\mathrm{C} 56-\mathrm{H} 56$ & 118.6 \\
\hline $\mathrm{N} 4-\mathrm{C} 5-\mathrm{C} 6$ & $112.1(2)$ & $\mathrm{C} 55-\mathrm{C} 56-\mathrm{H} 56$ & 118.6 \\
\hline $\mathrm{N} 4-\mathrm{C} 5-\mathrm{H} 5 \mathrm{~A}$ & 109.2 & $\mathrm{C} 54-\mathrm{O} 54-\mathrm{C} 57$ & $117.8(15)$ \\
\hline $\mathrm{C} 6-\mathrm{C} 5-\mathrm{H} 5 \mathrm{~A}$ & 109.2 & O54-C57-H57A & 109.5 \\
\hline $\mathrm{N} 4-\mathrm{C} 5-\mathrm{H} 5 \mathrm{~B}$ & 109.2 & O54-C57-H57B & 109.5 \\
\hline $\mathrm{C} 6-\mathrm{C} 5-\mathrm{H} 5 \mathrm{~B}$ & 109.2 & $\mathrm{H} 57 \mathrm{~A}-\mathrm{C} 57-\mathrm{H} 57 \mathrm{~B}$ & 109.5 \\
\hline $\mathrm{H} 5 \mathrm{~A}-\mathrm{C} 5-\mathrm{H} 5 \mathrm{~B}$ & 107.9 & $\mathrm{O} 54-\mathrm{C} 57-\mathrm{H} 57 \mathrm{C}$ & 109.5 \\
\hline $\mathrm{N} 1-\mathrm{C} 6-\mathrm{C} 5$ & $110.6(2)$ & $\mathrm{H} 57 \mathrm{~A}-\mathrm{C} 57-\mathrm{H} 57 \mathrm{C}$ & 109.5 \\
\hline $\mathrm{N} 1-\mathrm{C} 6-\mathrm{H} 6 \mathrm{~A}$ & 109.5 & $\mathrm{H} 57 \mathrm{~B}-\mathrm{C} 57-\mathrm{H} 57 \mathrm{C}$ & 109.5 \\
\hline $\mathrm{C} 5-\mathrm{C} 6-\mathrm{H} 6 \mathrm{~A}$ & 109.5 & $\mathrm{C} 36-\mathrm{C} 31-\mathrm{C} 32$ & $118.3(3)$ \\
\hline $\mathrm{N} 1-\mathrm{C} 6-\mathrm{H} 6 \mathrm{~B}$ & 109.5 & $\mathrm{C} 36-\mathrm{C} 31-\mathrm{C} 37$ & $121.4(3)$ \\
\hline $\mathrm{C} 5-\mathrm{C} 6-\mathrm{H} 6 \mathrm{~B}$ & 109.5 & $\mathrm{C} 32-\mathrm{C} 31-\mathrm{C} 37$ & $120.2(3)$ \\
\hline $\mathrm{H} 6 \mathrm{~A}-\mathrm{C} 6-\mathrm{H} 6 \mathrm{~B}$ & 108.1 & $\mathrm{C} 33-\mathrm{C} 32-\mathrm{C} 31$ & $121.0(3)$ \\
\hline $\mathrm{C} 26-\mathrm{C} 21-\mathrm{C} 22$ & $115.6(7)$ & $\mathrm{C} 33-\mathrm{C} 32-\mathrm{H} 32$ & 119.5 \\
\hline $\mathrm{C} 26-\mathrm{C} 21-\mathrm{N} 4$ & $121.3(11)$ & $\mathrm{C} 31-\mathrm{C} 32-\mathrm{H} 32$ & 119.5 \\
\hline $\mathrm{C} 22-\mathrm{C} 21-\mathrm{N} 4$ & $123.1(9)$ & $\mathrm{C} 34-\mathrm{C} 33-\mathrm{C} 32$ & $118.3(4)$ \\
\hline $\mathrm{C} 23-\mathrm{C} 22-\mathrm{C} 21$ & $121.3(4)$ & $\mathrm{C} 34-\mathrm{C} 33-\mathrm{H} 33$ & 120.8 \\
\hline $\mathrm{C} 23-\mathrm{C} 22-\mathrm{H} 22$ & 119.4 & $\mathrm{C} 32-\mathrm{C} 33-\mathrm{H} 33$ & 120.8 \\
\hline $\mathrm{C} 21-\mathrm{C} 22-\mathrm{H} 22$ & 119.4 & $\mathrm{C} 35-\mathrm{C} 34-\mathrm{F} 34$ & $119.1(4)$ \\
\hline $\mathrm{C} 24-\mathrm{C} 23-\mathrm{C} 22$ & $121.8(4)$ & $\mathrm{C} 35-\mathrm{C} 34-\mathrm{C} 33$ & $122.6(4)$ \\
\hline $\mathrm{C} 24-\mathrm{C} 23-\mathrm{H} 23$ & 119.1 & $\mathrm{~F} 34-\mathrm{C} 34-\mathrm{C} 33$ & $118.3(4)$ \\
\hline $\mathrm{C} 22-\mathrm{C} 23-\mathrm{H} 23$ & 119.1 & $\mathrm{C} 34-\mathrm{C} 35-\mathrm{C} 36$ & $118.6(3)$ \\
\hline $\mathrm{C} 23-\mathrm{C} 24-\mathrm{C} 25$ & $118.5(4)$ & $\mathrm{C} 34-\mathrm{C} 35-\mathrm{H} 35$ & 120.7 \\
\hline $\mathrm{C} 23-\mathrm{C} 24-\mathrm{O} 24$ & $116.6(4)$ & $\mathrm{C} 36-\mathrm{C} 35-\mathrm{H} 35$ & 120.7 \\
\hline $\mathrm{C} 25-\mathrm{C} 24-\mathrm{O} 24$ & $124.8(4)$ & $\mathrm{C} 35-\mathrm{C} 36-\mathrm{C} 31$ & $121.2(3)$ \\
\hline $\mathrm{C} 24-\mathrm{C} 25-\mathrm{C} 26$ & $119.5(6)$ & $\mathrm{C} 35-\mathrm{C} 36-\mathrm{H} 36$ & 119.4 \\
\hline $\mathrm{C} 24-\mathrm{C} 25-\mathrm{H} 25$ & 120.2 & $\mathrm{C} 31-\mathrm{C} 36-\mathrm{H} 36$ & 119.4 \\
\hline $\mathrm{C} 26-\mathrm{C} 25-\mathrm{H} 25$ & 120.2 & $\mathrm{O} 32-\mathrm{C} 37-\mathrm{O} 31$ & $123.5(4)$ \\
\hline $\mathrm{C} 21-\mathrm{C} 26-\mathrm{C} 25$ & $123.2(6)$ & $\mathrm{O} 32-\mathrm{C} 37-\mathrm{C} 31$ & $118.3(4)$ \\
\hline $\mathrm{C} 21-\mathrm{C} 26-\mathrm{H} 26$ & 118.4 & $\mathrm{O} 31-\mathrm{C} 37-\mathrm{C} 31$ & $118.2(3)$ \\
\hline $\mathrm{C} 25-\mathrm{C} 26-\mathrm{H} 26$ & 118.4 & $\mathrm{H} 41-\mathrm{O} 41-\mathrm{H} 42$ & $101(3)$ \\
\hline
\end{tabular}




$\begin{array}{ll}\mathrm{C} 24-\mathrm{O} 24-\mathrm{C} 27 & 118.8(4) \\ \mathrm{C} 6-\mathrm{N} 1-\mathrm{C} 2-\mathrm{C} 3 & -56.3(3) \\ \mathrm{N} 1-\mathrm{C} 2-\mathrm{C} 3-\mathrm{N} 4 & 55.3(3) \\ \mathrm{C} 2-\mathrm{C} 3-\mathrm{N} 4-\mathrm{C} 51 & 178(9) \\ \mathrm{C} 2-\mathrm{C} 3-\mathrm{N} 4-\mathrm{C} 21 & 174(2) \\ \mathrm{C} 2-\mathrm{C} 3-\mathrm{N} 4-\mathrm{C} 5 & -53.7(3) \\ \mathrm{C} 51-\mathrm{N} 4-\mathrm{C} 5-\mathrm{C} 6 & -174(8) \\ \mathrm{C} 21-\mathrm{N} 4-\mathrm{C} 5-\mathrm{C} 6 & -173(2) \\ \mathrm{C} 3-\mathrm{N} 4-\mathrm{C} 5-\mathrm{C} 6 & 54.6(3) \\ \mathrm{C} 2-\mathrm{N} 1-\mathrm{C} 6-\mathrm{C} 5 & 57.4(3) \\ \mathrm{N} 4-\mathrm{C} 5-\mathrm{C} 6-\mathrm{N} 1 & -57.2(3) \\ \mathrm{C} 3-\mathrm{N} 4-\mathrm{C} 21-\mathrm{C} 26 & 169(3) \\ \mathrm{C} 5-\mathrm{N} 4-\mathrm{C} 21-\mathrm{C} 26 & 38(4) \\ \mathrm{C} 3-\mathrm{N} 4-\mathrm{C} 21-\mathrm{C} 22 & -10(5) \\ \mathrm{C} 5-\mathrm{N} 4-\mathrm{C} 21-\mathrm{C} 22 & -140(3) \\ \mathrm{C} 26-\mathrm{C} 21-\mathrm{C} 22-\mathrm{C} 23 & 2(4) \\ \mathrm{N} 4-\mathrm{C} 21-\mathrm{C} 22-\mathrm{C} 23 & -179(2) \\ \mathrm{C} 21-\mathrm{C} 22-\mathrm{C} 23-\mathrm{C} 24 & -3(2) \\ \mathrm{C} 22-\mathrm{C} 23-\mathrm{C} 24-\mathrm{C} 25 & 2.3(13) \\ \mathrm{C} 22-\mathrm{C} 23-\mathrm{C} 24-\mathrm{O} 24 & -179.7(6) \\ \mathrm{C} 23-\mathrm{C} 24-\mathrm{C} 25-\mathrm{C} 26 & -2(2) \\ \mathrm{O} 24-\mathrm{C} 24-\mathrm{C} 25-\mathrm{C} 26 & -179.9(14) \\ \mathrm{C} 22-\mathrm{C} 21-\mathrm{C} 26-\mathrm{C} 25 & -2(4) \\ \mathrm{N} 4-\mathrm{C} 21-\mathrm{C} 26-\mathrm{C} 25 & 179(3) \\ \mathrm{C} 24-\mathrm{C} 25-\mathrm{C} 26-\mathrm{C} 21 & 2(3) \\ \mathrm{C} 23-\mathrm{C} 24-\mathrm{O} 24-\mathrm{C} 27 & 173.8(10) \\ \mathrm{C} 25-\mathrm{C} 24-\mathrm{O} 24-\mathrm{C} 27 & -8.4(16) \\ \mathrm{C} 3-\mathrm{N} 4-\mathrm{C} 51-\mathrm{C} 56 & 162(12) \\ \mathrm{C} 5-\mathrm{N} 4-\mathrm{C} 51-\mathrm{C} 56 & 34(18) \\ & \end{array}$

$\begin{array}{ll}\text { C3-N4-C51-C52 } & -38(19) \\ \text { C5-N4-C51-C52 } & -166(12) \\ \text { C56-C51-C52-C53 } & -9(19) \\ \text { N4-C51-C52-C53 } & -170(10) \\ \text { C51-C52-C53-C54 } & 8(11) \\ \text { C52-C53-C54-C55 } & -10(7) \\ \text { C52-C53-C54-O54 } & -175(4) \\ \text { C53-C54-C55-C56 } & 12(11) \\ \text { O54-C54-C55-C56 } & 176(7) \\ \text { N4-C51-C56-C55 } & 172(13) \\ \text { C54-C55-C56-C51 } & -14(15) \\ \text { C53-C54-O54-C57 } & 174(5) \\ \text { C55-C54-O54-C57 } & 10(8) \\ \text { C36-C31-C32-C33 } & 0.8(4) \\ \text { C37-C31-C32-C33 } & -179.3(3) \\ \text { C31-C32-C33-C34 } & 0.1(5) \\ \text { C32-C33-C34-C35 } & -0.7(5) \\ \text { C32-C33-C34-F34 } & 179.2(3) \\ \text { F34-C34-C35-C36 } & -179.4(3) \\ \text { C33-C34-C35-C36 } & 0.5(5) \\ \text { C34-C35-C36-C31 } & 0.4(5) \\ \text { C32-C31-C36-C35 } & -1.0(4) \\ \text { C37-C31-C36-C35 } & 179.1(3) \\ \text { C36-C31-C37-O32 } & 177.2(3) \\ \text { C32-C31-C37-O32 } & -2.7(4) \\ \text { C36-C31-C37-O31 } & -3.3(4) \\ \text { C32-C31-C37-O31 } & 176.8(3) \\ & \end{array}$

Hydrogen-bond geometry $\left(\AA,{ }^{\circ}\right)$

\begin{tabular}{lllll}
\hline$D-\mathrm{H} \cdots A$ & $D-\mathrm{H}$ & $\mathrm{H} \cdots A$ & $D \cdots A$ & $D-\mathrm{H} \cdots A$ \\
\hline $\mathrm{N} 1-\mathrm{H} 11 \cdots \mathrm{O} 31$ & $1.09(3)$ & $1.67(3)$ & $2.758(4)$ & $174.1(19)$ \\
$\mathrm{N} 1-\mathrm{H} 12 \cdots \mathrm{O} 41$ & $0.86(3)$ & $1.96(3)$ & $2.818(4)$ & $170(3)$ \\
$\mathrm{O} 41-\mathrm{H} 41 \cdots \mathrm{O} 32^{\mathrm{i}}$ & $0.86(4)$ & $1.75(4)$ & $2.627(4)$ & $174(4)$ \\
$\mathrm{O} 41-\mathrm{H} 42 \cdots \mathrm{O} 31^{\mathrm{ii}}$ & $0.91(4)$ & $1.88(4)$ & $2.768(3)$ & $163(3)$ \\
$\mathrm{C} 2-\mathrm{H} 2 B \cdots \mathrm{O} 31^{\mathrm{iii}}$ & 0.97 & 2.58 & $3.529(4)$ & 166 \\
$\mathrm{C} 6-\mathrm{H} 6 B \cdots \mathrm{O} 41^{\mathrm{i}}$ & 0.97 & 2.57 & $3.386(4)$ & 142 \\
$\mathrm{C} 22-\mathrm{H} 22 \cdots C g 1^{\mathrm{ii}}$ & 0.93 & 2.93 & $3.664(12)$ & 137 \\
$\mathrm{C} 26-\mathrm{H} 26 \cdots C g 1^{\text {iv }}$ & 0.93 & 2.81 & $3.56(2)$ & 138 \\
$\mathrm{C} 56-\mathrm{H} 56 \cdots C g 1^{\mathrm{iv}}$ & 0.93 & 2.96 & $3.55(9)$ & 123
\end{tabular}

Symmetry codes: (i) $-x+1,-y+1,-z+1$; (ii) $-x+2,-y+1,-z+1$; (iii) $-x+2,-y+2,-z+1$; (iv) $-x+1,-y+2,-z+1$. 
4-(4-Methoxyphenyl)piperazin-1-ium 4-chlorobenzoate monohydrate (III)

Crystal data

$\mathrm{C}_{11} \mathrm{H}_{17} \mathrm{~N}_{2} \mathrm{O}^{+} \cdot \mathrm{C}_{7} \mathrm{H}_{4} \mathrm{ClO}_{2}^{-} \cdot \mathrm{H}_{2} \mathrm{O}$

$M_{r}=366.83$

Triclinic, $P \overline{1}$

$a=6.211(1) \AA$

$b=7.481(1) \AA$

$c=20.144(4) \AA$

$\alpha=84.90(2)^{\circ}$

$\beta=87.48(2)^{\circ}$

$\gamma=85.19(2)^{\circ}$

$V=928.4(3) \AA^{3}$

\section{Data collection}

Oxford Diffraction Xcalibur with Sapphire CCD diffractometer

Radiation source: Enhance (Mo) X-ray Source

Graphite monochromator

$\omega$ scans

Absorption correction: multi-scan

(CrysAlis RED; Oxford Diffraction, 2009)

$T_{\min }=0.951, T_{\max }=0.995$

\section{Refinement}

Refinement on $F^{2}$

Least-squares matrix: full

$R\left[F^{2}>2 \sigma\left(F^{2}\right)\right]=0.065$

$w R\left(F^{2}\right)=0.135$

$S=0.94$

3454 reflections

265 parameters

17 restraints
$Z=2$

$F(000)=388$

$D_{\mathrm{x}}=1.312 \mathrm{Mg} \mathrm{m}^{-3}$

Mo $K \alpha$ radiation, $\lambda=0.71073 \AA$

Cell parameters from 3962 reflections

$\theta=2.8-28.8^{\circ}$

$\mu=0.23 \mathrm{~mm}^{-1}$

$T=293 \mathrm{~K}$

Plate, colourless

$0.20 \times 0.16 \times 0.02 \mathrm{~mm}$

5883 measured reflections

3454 independent reflections

1343 reflections with $I>2 \sigma(I)$

$R_{\text {int }}=0.041$

$\theta_{\max }=25.5^{\circ}, \theta_{\min }=2.8^{\circ}$

$h=-7 \rightarrow 7$

$k=-9 \rightarrow 8$

$l=-24 \rightarrow 22$

Primary atom site location: difference Fourier map

Hydrogen site location: mixed

$\mathrm{H}$ atoms treated by a mixture of independent and constrained refinement

$w=1 /\left[\sigma^{2}\left(F_{\mathrm{o}}^{2}\right)+(0.0492 P)^{2}\right]$

where $P=\left(F_{\mathrm{o}}^{2}+2 F_{\mathrm{c}}^{2}\right) / 3$

$(\Delta / \sigma)_{\max }<0.001$

$\Delta \rho_{\max }=0.24 \mathrm{e} \AA^{-3}$

$\Delta \rho_{\min }=-0.23$ e $\AA^{-3}$

Special details

Experimental. Compound (III). IR ( $\left.\mathrm{KBr}, \mathrm{cm}^{-1}\right) 3320(\mathrm{OH}), 3003\left(\mathrm{NH}_{2}\right), 2837\left(\mathrm{OCH}_{3}\right), 1582(\mathrm{COO}), 772(\mathrm{CCl})$. NMR $\left(\mathrm{CDCl}_{3}\right) \delta\left({ }^{1} \mathrm{H}\right) 3.23\left(\mathrm{~m}, 4 \mathrm{H}\right.$, piperazine), $3.28(\mathrm{~m}, 4 \mathrm{H}$, piperazine $), 3.77\left(\mathrm{~s}, 3 \mathrm{H}, \mathrm{OCH}_{3}\right), 6.86(\mathrm{~m}, 4 \mathrm{H}$, methoxyphenyl), 7.36 (d, J = 8.4 Hz, 2H, chlorophenyl), 7.98 (d, J = 8.4 Hz,2H, chlorophenyl).

Geometry. All esds (except the esd in the dihedral angle between two 1.s. planes) are estimated using the full covariance matrix. The cell esds are taken into account individually in the estimation of esds in distances, angles and torsion angles; correlations between esds in cell parameters are only used when they are defined by crystal symmetry. An approximate (isotropic) treatment of cell esds is used for estimating esds involving l.s. planes.

Fractional atomic coordinates and isotropic or equivalent isotropic displacement parameters $\left(\AA^{2}\right)$

\begin{tabular}{llllll}
\hline & $x$ & $y$ & $z$ & $U_{\text {iso }} * U_{\text {eq }}$ & Occ. $(<1)$ \\
\hline N1 & $0.7833(5)$ & $0.7442(4)$ & $0.45388(15)$ & $0.0536(9)$ & \\
H11 & $0.827(5)$ & $0.777(4)$ & $0.5028(16)$ & $0.064^{*}$ & \\
H12 & $0.760(5)$ & $0.636(4)$ & $0.4593(16)$ & $0.064^{*}$ & \\
C2 & $0.9718(6)$ & $0.7498(5)$ & $0.40636(16)$ & $0.0570(10)$ & \\
H2A & 1.0888 & 0.6667 & 0.4234 & $0.068^{*}$ &
\end{tabular}




\begin{tabular}{|c|c|c|c|c|c|}
\hline $\mathrm{H} 2 \mathrm{~B}$ & 1.0216 & 0.8699 & 0.4020 & $0.068 *$ & \\
\hline C3 & $0.9136(6)$ & $0.6998(4)$ & $0.33895(16)$ & $0.0545(10)$ & \\
\hline $\mathrm{H} 3 \mathrm{~A}$ & 1.0371 & 0.7116 & 0.3082 & $0.065^{*}$ & \\
\hline H3B & 0.8803 & 0.5749 & 0.3427 & $0.065^{*}$ & \\
\hline N4 & $0.7287(4)$ & $0.8126(3)$ & $0.31215(12)$ & $0.0433(7)$ & \\
\hline C5 & $0.5425(5)$ & $0.8110(4)$ & $0.35968(15)$ & $0.0516(9)$ & \\
\hline H5A & 0.4918 & 0.6912 & 0.3650 & $0.062 *$ & \\
\hline H5B & 0.4260 & 0.8935 & 0.3420 & $0.062 *$ & \\
\hline C6 & $0.5992(6)$ & $0.8644(4)$ & $0.42667(15)$ & $0.0551(10)$ & \\
\hline H6A & 0.6377 & 0.9880 & 0.4222 & $0.066^{*}$ & \\
\hline H6B & 0.4747 & 0.8569 & 0.4573 & $0.066^{*}$ & \\
\hline $\mathrm{C} 21$ & $0.685(4)$ & $0.783(9)$ & $0.2449(9)$ & $0.0446(14)$ & $0.73(2)$ \\
\hline $\mathrm{C} 22$ & $0.8261(13)$ & 0.6777 (19) & $0.2052(4)$ & $0.060(3)$ & $0.73(2)$ \\
\hline $\mathrm{H} 22$ & 0.9523 & 0.6211 & 0.2230 & $0.072 *$ & $0.73(2)$ \\
\hline $\mathrm{C} 23$ & 0.7808 (13) & $0.657(2)$ & $0.1401(4)$ & $0.074(3)$ & $0.73(2)$ \\
\hline $\mathrm{H} 23$ & 0.8807 & 0.5916 & 0.1141 & $0.088^{*}$ & $0.73(2)$ \\
\hline $\mathrm{C} 24$ & $0.5923(18)$ & $0.730(3)$ & $0.1130(5)$ & $0.065(4)$ & $0.73(2)$ \\
\hline $\mathrm{C} 25$ & $0.450(2)$ & $0.828(5)$ & $0.1516(8)$ & $0.075(3)$ & $0.73(2)$ \\
\hline $\mathrm{H} 25$ & 0.3196 & 0.8781 & 0.1341 & $0.089 *$ & $0.73(2)$ \\
\hline $\mathrm{C} 26$ & $0.496(2)$ & $0.854(3)$ & $0.2162(6)$ & $0.060(3)$ & $0.73(2)$ \\
\hline $\mathrm{H} 26$ & 0.3958 & 0.9221 & 0.2413 & $0.072 *$ & $0.73(2)$ \\
\hline $\mathrm{O} 24$ & $0.564(2)$ & $0.697(2)$ & $0.0482(6)$ & $0.104(4)$ & $0.73(2)$ \\
\hline $\mathrm{C} 27$ & $0.362(3)$ & $0.743(2)$ & $0.0203(7)$ & $0.101(4)$ & $0.73(2)$ \\
\hline $\mathrm{H} 27 \mathrm{~A}$ & 0.2517 & 0.6892 & 0.0485 & $0.152 *$ & $0.73(2)$ \\
\hline $\mathrm{H} 27 \mathrm{~B}$ & 0.3623 & 0.6989 & -0.0231 & $0.152^{*}$ & $0.73(2)$ \\
\hline $\mathrm{H} 27 \mathrm{C}$ & 0.3331 & 0.8713 & 0.0164 & $0.152^{*}$ & $0.73(2)$ \\
\hline $\mathrm{C} 51$ & $0.668(10)$ & $0.79(3)$ & $0.247(2)$ & $0.0446(14)$ & $0.27(2)$ \\
\hline C52 & $0.833(4)$ & $0.755(4)$ & $0.1991(13)$ & $0.060(3)$ & $0.27(2)$ \\
\hline H52 & 0.9761 & 0.7413 & 0.2112 & $0.072 *$ & $0.27(2)$ \\
\hline C53 & $0.783(4)$ & $0.739(4)$ & $0.1340(12)$ & $0.074(3)$ & $0.27(2)$ \\
\hline H53 & 0.8924 & 0.7021 & 0.1039 & $0.088^{*}$ & $0.27(2)$ \\
\hline $\mathrm{C} 54$ & $0.577(5)$ & $0.775(9)$ & $0.1126(15)$ & $0.065(4)$ & $0.27(2)$ \\
\hline C55 & $0.418(6)$ & $0.825(15)$ & $0.158(2)$ & $0.075(3)$ & $0.27(2)$ \\
\hline H55 & 0.2775 & 0.8534 & 0.1437 & $0.089^{*}$ & $0.27(2)$ \\
\hline C56 & $0.464(5)$ & $0.834(8)$ & 0.2235 (18) & $0.060(3)$ & $0.27(2)$ \\
\hline H56 & 0.3532 & 0.8701 & 0.2531 & $0.072 *$ & $0.27(2)$ \\
\hline O54 & $0.548(7)$ & $0.751(7)$ & $0.0468(16)$ & $0.104(4)$ & $0.27(2)$ \\
\hline C57 & $0.351(8)$ & $0.814(6)$ & $0.018(2)$ & $0.101(4)$ & $0.27(2)$ \\
\hline H57A & 0.2346 & 0.7622 & 0.0438 & $0.152^{*}$ & $0.27(2)$ \\
\hline H57B & 0.3518 & 0.7814 & -0.0267 & $0.152 *$ & $0.27(2)$ \\
\hline $\mathrm{H} 57 \mathrm{C}$ & 0.3326 & 0.9432 & 0.0182 & $0.152^{*}$ & $0.27(2)$ \\
\hline C31 & $0.8074(6)$ & $0.7372(4)$ & $0.69495(18)$ & $0.0470(9)$ & \\
\hline C32 & $0.6605(7)$ & $0.6665(5)$ & $0.7412(2)$ & $0.0704(11)$ & \\
\hline H32 & 0.5407 & 0.6164 & 0.7267 & $0.084^{*}$ & \\
\hline C33 & $0.6886(8)$ & 0.6688 & $0.8091(2)$ & $0.0834(13)$ & \\
\hline H33 & 0.5883 & 0.6212 & 0.8400 & $0.100 *$ & \\
\hline C34 & $0.8662(9)$ & $0.7424(6)$ & $0.8301(2)$ & $0.0770(13)$ & \\
\hline $\mathrm{Cl34}$ & $0.8970(3)$ & 0.74735 (19) & $0.91487(5)$ & $0.1383(7)$ & \\
\hline
\end{tabular}




\begin{tabular}{lllll}
$\mathrm{C} 35$ & $1.0144(7)$ & $0.8114(5)$ & $0.7851(2)$ & $0.0695(11)$ \\
$\mathrm{H} 35$ & 1.1350 & 0.8601 & 0.7997 & $0.083^{*}$ \\
$\mathrm{C} 36$ & $0.9845(6)$ & $0.8084(4)$ & $0.71778(17)$ & $0.0543(10)$ \\
$\mathrm{H} 36$ & 1.0861 & 0.8555 & 0.6872 & $0.065^{*}$ \\
$\mathrm{C} 37$ & $0.7721(7)$ & $0.7351(5)$ & $0.6222(2)$ & $0.0581(11)$ \\
$\mathrm{O} 31$ & $0.9006(5)$ & $0.8125(3)$ & $0.58079(12)$ & $0.0697(8)$ \\
$\mathrm{O} 32$ & $0.6183(5)$ & $0.6568(4)$ & $0.60573(15)$ & $0.1085(11)$ \\
$\mathrm{O} 41$ & $0.7230(5)$ & $0.3744(3)$ & $0.46334(13)$ & $0.0683(8)$ \\
$\mathrm{H} 41$ & $0.614(7)$ & $0.363(5)$ & $0.4360(19)$ & $0.102^{*}$ \\
$\mathrm{H} 42$ & $0.846(7)$ & $0.315(5)$ & $0.4434(19)$ & $0.102^{*}$ \\
\hline
\end{tabular}

Atomic displacement parameters $\left(\AA^{2}\right)$

\begin{tabular}{|c|c|c|c|c|c|c|}
\hline & $U^{11}$ & $U^{22}$ & $U^{33}$ & $U^{12}$ & $U^{13}$ & $U^{23}$ \\
\hline N1 & $0.065(2)$ & $0.0467(18)$ & 0.0505 (19) & $-0.0043(19)$ & $-0.0122(18)$ & $-0.0079(17)$ \\
\hline $\mathrm{C} 2$ & $0.044(2)$ & $0.069(3)$ & $0.057(2)$ & -0.003 & -0.008 & $-0.0023(19)$ \\
\hline $\mathrm{C} 3$ & $0.043(2)$ & $0.065(2)$ & $0.053(2)$ & $0.007(2)$ & $-0.0072(19)$ & $-0.0050(18)$ \\
\hline N4 & $0.0390(19)$ & $0.0473(17)$ & $0.0432(17)$ & $0.0009(15)$ & $-0.0023(15)$ & $-0.0044(13)$ \\
\hline $\mathrm{C} 5$ & $0.049(2)$ & $0.055(2)$ & $0.049(2)$ & $0.0054(19)$ & 0.0007 (19) & $-0.0041(18)$ \\
\hline C6 & $0.057(3)$ & $0.056(2)$ & $0.051(2)$ & $0.006(2)$ & -0.0009 (19) & $-0.0052(18)$ \\
\hline $\mathrm{C} 21$ & $0.038(4)$ & $0.046(7)$ & $0.049(3)$ & $0.001(5)$ & $0.001(3)$ & $-0.005(2)$ \\
\hline $\mathrm{C} 22$ & $0.054(3)$ & $0.064(8)$ & $0.059(3)$ & $0.021(4)$ & $-0.007(2)$ & $-0.011(4)$ \\
\hline $\mathrm{C} 23$ & $0.073(4)$ & $0.084(9)$ & $0.059(3)$ & $0.035(5)$ & $-0.002(3)$ & $-0.020(5)$ \\
\hline $\mathrm{C} 24$ & $0.074(4)$ & $0.077(12)$ & $0.043(2)$ & $0.008(3)$ & $-0.004(2)$ & $-0.016(4)$ \\
\hline $\mathrm{C} 25$ & $0.064(5)$ & $0.104(4)$ & $0.053(4)$ & $0.021(7)$ & $-0.013(4)$ & $-0.013(5)$ \\
\hline $\mathrm{C} 26$ & $0.048(4)$ & $0.081(6)$ & $0.049(4)$ & $0.012(5)$ & 0.003 & $-0.012(4)$ \\
\hline $\mathrm{O} 24$ & $0.104(3)$ & $0.148(12)$ & $0.059(2)$ & $0.039(5)$ & $-0.0194(19)$ & $-0.038(4)$ \\
\hline $\mathrm{C} 27$ & $0.109(5)$ & $0.125(13)$ & $0.070(3)$ & $0.022(7)$ & $-0.034(3)$ & $-0.025(7)$ \\
\hline $\mathrm{C} 51$ & $0.038(4)$ & $0.046(7)$ & 0.049 (3) & $0.001(5)$ & $0.001(3)$ & $-0.005(2)$ \\
\hline C52 & $0.054(3)$ & $0.064(8)$ & $0.059(3)$ & $0.021(4)$ & $-0.007(2)$ & $-0.011(4)$ \\
\hline $\mathrm{C} 53$ & $0.073(4)$ & $0.084(9)$ & $0.059(3)$ & $0.035(5)$ & $-0.002(3)$ & $-0.020(5)$ \\
\hline $\mathrm{C} 54$ & $0.074(4)$ & $0.077(12)$ & $0.043(2)$ & $0.008(3)$ & $-0.004(2)$ & $-0.016(4)$ \\
\hline $\mathrm{C} 55$ & $0.064(5)$ & $0.104(4)$ & $0.053(4)$ & $0.021(7)$ & $-0.013(4)$ & $-0.013(5)$ \\
\hline $\mathrm{C} 56$ & $0.048(4)$ & $0.081(6)$ & 0.049 (4) & $0.012(5)$ & $0.003(3)$ & $-0.012(4)$ \\
\hline $\mathrm{O} 54$ & $0.104(3)$ & $0.148(12)$ & $0.059(2)$ & $0.039(5)$ & $-0.0194(19)$ & $-0.038(4)$ \\
\hline C57 & $0.109(5)$ & $0.125(13)$ & 0.070 & $0.022(7)$ & $-0.034(3)$ & $-0.025(7)$ \\
\hline $\mathrm{C} 31$ & $0.042(2)$ & $0.040(2)$ & $0.059(2)$ & $0.0033(19)$ & $-0.006(2)$ & $-0.0082(18)$ \\
\hline $\mathrm{C} 32$ & $0.061(3)$ & $0.061(3)$ & $0.090(3)$ & $-0.008(2)$ & $0.005(3)$ & $-0.012(2)$ \\
\hline $\mathrm{C} 33$ & $0.080(4)$ & $0.085(3)$ & $0.080(4)$ & $-0.001(3)$ & $0.029(3)$ & $0.002(3)$ \\
\hline C34 & $0.089(4)$ & $0.078(3)$ & $0.060(3)$ & $0.017(3)$ & $-0.007(3)$ & $-0.005(2)$ \\
\hline $\mathrm{Cl} 34$ & $0.1856(16)$ & $0.1630(13)$ & $0.0572(8)$ & $0.0402(11)$ & $-0.0065(8)$ & $-0.0083(8)$ \\
\hline $\mathrm{C} 35$ & $0.070(3)$ & $0.077(3)$ & $0.062(3)$ & $-0.002(2)$ & $-0.015(3)$ & $-0.010(2)$ \\
\hline C36 & $0.054(3)$ & $0.054(2)$ & 0.055 & $-0.007(2)$ & $-0.007(2)$ & $-0.0026(18)$ \\
\hline C37 & $0.056(3)$ & $0.044(2)$ & 0.075 & $0.007(2)$ & $-0.017(3)$ & $-0.017(2)$ \\
\hline $\mathrm{O} 31$ & $0.087(2)$ & $0.0683(18)$ & $0.0553(17)$ & $-0.0048(16)$ & $-0.0119(16)$ & $-0.0086(14)$ \\
\hline $\mathrm{O} 32$ & $0.095(2)$ & $0.132(3)$ & $0.110(2)$ & $-0.033(2)$ & $-0.040(2)$ & $-0.0309(19)$ \\
\hline $\mathrm{O} 41$ & $0.068(2)$ & $0.0661(17)$ & $0.0737(19)$ & $-0.0116(16)$ & $-0.0191(15)$ & $-0.0057(14)$ \\
\hline
\end{tabular}


Geometric parameters $\left(\AA,{ }^{\circ}\right)$

\begin{tabular}{|c|c|c|c|}
\hline $\mathrm{N} 1-\mathrm{C} 2$ & $1.481(4)$ & $\mathrm{C} 27-\mathrm{H} 27 \mathrm{C}$ & 0.9600 \\
\hline $\mathrm{N} 1-\mathrm{C} 6$ & $1.487(4)$ & C51-C56 & $1.376(12)$ \\
\hline N1-H11 & $1.09(3)$ & $\mathrm{C} 51-\mathrm{C} 52$ & $1.40(2)$ \\
\hline $\mathrm{N} 1-\mathrm{H} 12$ & $0.83(3)$ & $\mathrm{C} 52-\mathrm{C} 53$ & $1.378(9)$ \\
\hline $\mathrm{C} 2-\mathrm{C} 3$ & $1.507(4)$ & C52-H52 & 0.9300 \\
\hline $\mathrm{C} 2-\mathrm{H} 2 \mathrm{~A}$ & 0.9700 & $\mathrm{C} 53-\mathrm{C} 54$ & $1.366(9)$ \\
\hline $\mathrm{C} 2-\mathrm{H} 2 \mathrm{~B}$ & 0.9700 & $\mathrm{C} 53-\mathrm{H} 53$ & 0.9300 \\
\hline $\mathrm{C} 3-\mathrm{N} 4$ & $1.461(4)$ & $\mathrm{C} 54-\mathrm{C} 55$ & $1.363(14)$ \\
\hline $\mathrm{C} 3-\mathrm{H} 3 \mathrm{~A}$ & 0.9700 & $\mathrm{C} 54-\mathrm{O} 54$ & $1.373(8)$ \\
\hline C $3-\mathrm{H} 3 \mathrm{~B}$ & 0.9700 & $\mathrm{C} 55-\mathrm{C} 56$ & $1.380(9)$ \\
\hline $\mathrm{N} 4-\mathrm{C} 51$ & $1.40(3)$ & C55-H55 & 0.9300 \\
\hline $\mathrm{N} 4-\mathrm{C} 21$ & $1.434(12)$ & C56-H56 & 0.9300 \\
\hline $\mathrm{N} 4-\mathrm{C} 5$ & $1.468(4)$ & $\mathrm{O} 54-\mathrm{C} 57$ & $1.401(9)$ \\
\hline $\mathrm{C} 5-\mathrm{C} 6$ & $1.505(4)$ & C57-H57A & 0.9600 \\
\hline $\mathrm{C} 5-\mathrm{H} 5 \mathrm{~A}$ & 0.9700 & C57-H57B & 0.9600 \\
\hline C5-H5B & 0.9700 & $\mathrm{C} 57-\mathrm{H} 57 \mathrm{C}$ & 0.9600 \\
\hline C6-H6A & 0.9700 & $\mathrm{C} 31-\mathrm{C} 36$ & $1.376(4)$ \\
\hline C6-H6B & 0.9700 & $\mathrm{C} 31-\mathrm{C} 32$ & $1.378(5)$ \\
\hline $\mathrm{C} 21-\mathrm{C} 26$ & $1.376(12)$ & $\mathrm{C} 31-\mathrm{C} 37$ & $1.494(5)$ \\
\hline $\mathrm{C} 21-\mathrm{C} 22$ & $1.40(2)$ & $\mathrm{C} 32-\mathrm{C} 33$ & $1.389(5)$ \\
\hline $\mathrm{C} 22-\mathrm{C} 23$ & $1.378(5)$ & $\mathrm{C} 32-\mathrm{H} 32$ & 0.9300 \\
\hline $\mathrm{C} 22-\mathrm{H} 22$ & 0.9300 & $\mathrm{C} 33-\mathrm{C} 34$ & $1.372(5)$ \\
\hline $\mathrm{C} 23-\mathrm{C} 24$ & $1.366(6)$ & C $33-\mathrm{H} 33$ & 0.9300 \\
\hline $\mathrm{C} 23-\mathrm{H} 23$ & 0.9300 & $\mathrm{C} 34-\mathrm{C} 35$ & $1.363(5)$ \\
\hline $\mathrm{C} 24-\mathrm{C} 25$ & $1.363(12)$ & $\mathrm{C} 34-\mathrm{Cl} 34$ & $1.731(4)$ \\
\hline $\mathrm{C} 24-\mathrm{O} 24$ & $1.373(5)$ & $\mathrm{C} 35-\mathrm{C} 36$ & $1.379(4)$ \\
\hline $\mathrm{C} 25-\mathrm{C} 26$ & $1.380(6)$ & C35-H35 & 0.9300 \\
\hline $\mathrm{C} 25-\mathrm{H} 25$ & 0.9300 & C36-H36 & 0.9300 \\
\hline $\mathrm{C} 26-\mathrm{H} 26$ & 0.9300 & $\mathrm{C} 37-\mathrm{O} 32$ & $1.233(4)$ \\
\hline $\mathrm{O} 24-\mathrm{C} 27$ & $1.400(6)$ & $\mathrm{C} 37-\mathrm{O} 31$ & $1.265(4)$ \\
\hline $\mathrm{C} 27-\mathrm{H} 27 \mathrm{~A}$ & 0.9600 & $\mathrm{O} 41-\mathrm{H} 41$ & $0.91(4)$ \\
\hline $\mathrm{C} 27-\mathrm{H} 27 \mathrm{~B}$ & 0.9600 & $\mathrm{O} 41-\mathrm{H} 42$ & $0.94(4)$ \\
\hline $\mathrm{C} 2-\mathrm{N} 1-\mathrm{C} 6$ & $110.0(3)$ & $\mathrm{O} 24-\mathrm{C} 27-\mathrm{H} 27 \mathrm{~A}$ & 109.5 \\
\hline $\mathrm{C} 2-\mathrm{N} 1-\mathrm{H} 11$ & $110.7(16)$ & $\mathrm{O} 24-\mathrm{C} 27-\mathrm{H} 27 \mathrm{~B}$ & 109.5 \\
\hline $\mathrm{C} 6-\mathrm{N} 1-\mathrm{H} 11$ & $112.6(15)$ & $\mathrm{H} 27 \mathrm{~A}-\mathrm{C} 27-\mathrm{H} 27 \mathrm{~B}$ & 109.5 \\
\hline $\mathrm{C} 2-\mathrm{N} 1-\mathrm{H} 12$ & $105(2)$ & $\mathrm{O} 24-\mathrm{C} 27-\mathrm{H} 27 \mathrm{C}$ & 109.5 \\
\hline $\mathrm{C} 6-\mathrm{N} 1-\mathrm{H} 12$ & $114(2)$ & $\mathrm{H} 27 \mathrm{~A}-\mathrm{C} 27-\mathrm{H} 27 \mathrm{C}$ & 109.5 \\
\hline $\mathrm{H} 11-\mathrm{N} 1-\mathrm{H} 12$ & $105(3)$ & $\mathrm{H} 27 \mathrm{~B}-\mathrm{C} 27-\mathrm{H} 27 \mathrm{C}$ & 109.5 \\
\hline $\mathrm{N} 1-\mathrm{C} 2-\mathrm{C} 3$ & $110.9(3)$ & $\mathrm{C} 56-\mathrm{C} 51-\mathrm{C} 52$ & $116.1(16)$ \\
\hline $\mathrm{N} 1-\mathrm{C} 2-\mathrm{H} 2 \mathrm{~A}$ & 109.5 & $\mathrm{C} 56-\mathrm{C} 51-\mathrm{N} 4$ & $125(2)$ \\
\hline $\mathrm{C} 3-\mathrm{C} 2-\mathrm{H} 2 \mathrm{~A}$ & 109.5 & $\mathrm{C} 52-\mathrm{C} 51-\mathrm{N} 4$ & $118(4)$ \\
\hline $\mathrm{N} 1-\mathrm{C} 2-\mathrm{H} 2 \mathrm{~B}$ & 109.5 & $\mathrm{C} 53-\mathrm{C} 52-\mathrm{C} 51$ & $120.5(14)$ \\
\hline $\mathrm{C} 3-\mathrm{C} 2-\mathrm{H} 2 \mathrm{~B}$ & 109.5 & $\mathrm{C} 53-\mathrm{C} 52-\mathrm{H} 52$ & 119.7 \\
\hline $\mathrm{H} 2 \mathrm{~A}-\mathrm{C} 2-\mathrm{H} 2 \mathrm{~B}$ & 108.1 & $\mathrm{C} 51-\mathrm{C} 52-\mathrm{H} 52$ & 119.7 \\
\hline $\mathrm{N} 4-\mathrm{C} 3-\mathrm{C} 2$ & $112.4(3)$ & $\mathrm{C} 54-\mathrm{C} 53-\mathrm{C} 52$ & $121.5(11)$ \\
\hline
\end{tabular}




\begin{tabular}{|c|c|c|c|}
\hline $\mathrm{N} 4-\mathrm{C} 3-\mathrm{H} 3 \mathrm{~A}$ & 109.1 & $\mathrm{C} 54-\mathrm{C} 53-\mathrm{H} 53$ & 119.2 \\
\hline $\mathrm{C} 2-\mathrm{C} 3-\mathrm{H} 3 \mathrm{~A}$ & 109.1 & $\mathrm{C} 52-\mathrm{C} 53-\mathrm{H} 53$ & 119.2 \\
\hline $\mathrm{N} 4-\mathrm{C} 3-\mathrm{H} 3 \mathrm{~B}$ & 109.1 & $\mathrm{C} 55-\mathrm{C} 54-\mathrm{C} 53$ & $118.5(10)$ \\
\hline $\mathrm{C} 2-\mathrm{C} 3-\mathrm{H} 3 \mathrm{~B}$ & 109.1 & $\mathrm{C} 55-\mathrm{C} 54-\mathrm{O} 54$ & $125.5(13)$ \\
\hline $\mathrm{H} 3 \mathrm{~A}-\mathrm{C} 3-\mathrm{H} 3 \mathrm{~B}$ & 107.9 & $\mathrm{C} 53-\mathrm{C} 54-\mathrm{O} 54$ & $116.0(12)$ \\
\hline $\mathrm{C} 51-\mathrm{N} 4-\mathrm{C} 3$ & $118(4)$ & $\mathrm{C} 54-\mathrm{C} 55-\mathrm{C} 56$ & $120.5(12)$ \\
\hline $\mathrm{C} 21-\mathrm{N} 4-\mathrm{C} 3$ & $113.7(13)$ & $\mathrm{C} 54-\mathrm{C} 55-\mathrm{H} 55$ & 119.8 \\
\hline $\mathrm{C} 51-\mathrm{N} 4-\mathrm{C} 5$ & $112(4)$ & $\mathrm{C} 56-\mathrm{C} 55-\mathrm{H} 55$ & 119.8 \\
\hline $\mathrm{C} 21-\mathrm{N} 4-\mathrm{C} 5$ & $115.8(14)$ & $\mathrm{C} 51-\mathrm{C} 56-\mathrm{C} 55$ & $122.4(12)$ \\
\hline $\mathrm{C} 3-\mathrm{N} 4-\mathrm{C} 5$ & $111.1(2)$ & $\mathrm{C} 51-\mathrm{C} 56-\mathrm{H} 56$ & 118.8 \\
\hline $\mathrm{N} 4-\mathrm{C} 5-\mathrm{C} 6$ & $111.8(3)$ & $\mathrm{C} 55-\mathrm{C} 56-\mathrm{H} 56$ & 118.8 \\
\hline $\mathrm{N} 4-\mathrm{C} 5-\mathrm{H} 5 \mathrm{~A}$ & 109.3 & $\mathrm{C} 54-\mathrm{O} 54-\mathrm{C} 57$ & $119.2(14)$ \\
\hline $\mathrm{C} 6-\mathrm{C} 5-\mathrm{H} 5 \mathrm{~A}$ & 109.3 & $\mathrm{O} 54-\mathrm{C} 57-\mathrm{H} 57 \mathrm{~A}$ & 109.5 \\
\hline $\mathrm{N} 4-\mathrm{C} 5-\mathrm{H} 5 \mathrm{~B}$ & 109.3 & O54-C57-H57B & 109.5 \\
\hline $\mathrm{C} 6-\mathrm{C} 5-\mathrm{H} 5 \mathrm{~B}$ & 109.3 & $\mathrm{H} 57 \mathrm{~A}-\mathrm{C} 57-\mathrm{H} 57 \mathrm{~B}$ & 109.5 \\
\hline $\mathrm{H} 5 \mathrm{~A}-\mathrm{C} 5-\mathrm{H} 5 \mathrm{~B}$ & 107.9 & $\mathrm{O} 54-\mathrm{C} 57-\mathrm{H} 57 \mathrm{C}$ & 109.5 \\
\hline $\mathrm{N} 1-\mathrm{C} 6-\mathrm{C} 5$ & $110.5(3)$ & $\mathrm{H} 57 \mathrm{~A}-\mathrm{C} 57-\mathrm{H} 57 \mathrm{C}$ & 109.5 \\
\hline $\mathrm{N} 1-\mathrm{C} 6-\mathrm{H} 6 \mathrm{~A}$ & 109.6 & $\mathrm{H} 57 \mathrm{~B}-\mathrm{C} 57-\mathrm{H} 57 \mathrm{C}$ & 109.5 \\
\hline $\mathrm{C} 5-\mathrm{C} 6-\mathrm{H} 6 \mathrm{~A}$ & 109.6 & $\mathrm{C} 36-\mathrm{C} 31-\mathrm{C} 32$ & $118.3(3)$ \\
\hline $\mathrm{N} 1-\mathrm{C} 6-\mathrm{H} 6 \mathrm{~B}$ & 109.6 & $\mathrm{C} 36-\mathrm{C} 31-\mathrm{C} 37$ & $121.7(4)$ \\
\hline $\mathrm{C} 5-\mathrm{C} 6-\mathrm{H} 6 \mathrm{~B}$ & 109.6 & $\mathrm{C} 32-\mathrm{C} 31-\mathrm{C} 37$ & $120.0(4)$ \\
\hline $\mathrm{H} 6 \mathrm{~A}-\mathrm{C} 6-\mathrm{H} 6 \mathrm{~B}$ & 108.1 & $\mathrm{C} 31-\mathrm{C} 32-\mathrm{C} 33$ & $121.0(4)$ \\
\hline $\mathrm{C} 26-\mathrm{C} 21-\mathrm{C} 22$ & $116.2(8)$ & $\mathrm{C} 31-\mathrm{C} 32-\mathrm{H} 32$ & 119.5 \\
\hline $\mathrm{C} 26-\mathrm{C} 21-\mathrm{N} 4$ & $121.0(15)$ & $\mathrm{C} 33-\mathrm{C} 32-\mathrm{H} 32$ & 119.5 \\
\hline $\mathrm{C} 22-\mathrm{C} 21-\mathrm{N} 4$ & $122.8(12)$ & $\mathrm{C} 34-\mathrm{C} 33-\mathrm{C} 32$ & $119.1(4)$ \\
\hline $\mathrm{C} 23-\mathrm{C} 22-\mathrm{C} 21$ & $120.9(6)$ & $\mathrm{C} 34-\mathrm{C} 33-\mathrm{H} 33$ & 120.4 \\
\hline $\mathrm{C} 23-\mathrm{C} 22-\mathrm{H} 22$ & 119.5 & $\mathrm{C} 32-\mathrm{C} 33-\mathrm{H} 33$ & 120.4 \\
\hline $\mathrm{C} 21-\mathrm{C} 22-\mathrm{H} 22$ & 119.5 & $\mathrm{C} 35-\mathrm{C} 34-\mathrm{C} 33$ & $120.7(4)$ \\
\hline $\mathrm{C} 24-\mathrm{C} 23-\mathrm{C} 22$ & $121.4(5)$ & $\mathrm{C} 35-\mathrm{C} 34-\mathrm{Cl} 34$ & $120.7(4)$ \\
\hline $\mathrm{C} 24-\mathrm{C} 23-\mathrm{H} 23$ & 119.3 & $\mathrm{C} 33-\mathrm{C} 34-\mathrm{Cl} 34$ & $118.6(4)$ \\
\hline $\mathrm{C} 22-\mathrm{C} 23-\mathrm{H} 23$ & 119.3 & $\mathrm{C} 34-\mathrm{C} 35-\mathrm{C} 36$ & $119.6(4)$ \\
\hline $\mathrm{C} 25-\mathrm{C} 24-\mathrm{C} 23$ & $118.5(5)$ & $\mathrm{C} 34-\mathrm{C} 35-\mathrm{H} 35$ & 120.2 \\
\hline $\mathrm{C} 25-\mathrm{C} 24-\mathrm{O} 24$ & $125.6(6)$ & $\mathrm{C} 36-\mathrm{C} 35-\mathrm{H} 35$ & 120.2 \\
\hline $\mathrm{C} 23-\mathrm{C} 24-\mathrm{O} 24$ & $115.9(6)$ & $\mathrm{C} 31-\mathrm{C} 36-\mathrm{C} 35$ & $121.3(4)$ \\
\hline $\mathrm{C} 24-\mathrm{C} 25-\mathrm{C} 26$ & $120.7(8)$ & $\mathrm{C} 31-\mathrm{C} 36-\mathrm{H} 36$ & 119.4 \\
\hline $\mathrm{C} 24-\mathrm{C} 25-\mathrm{H} 25$ & 119.7 & $\mathrm{C} 35-\mathrm{C} 36-\mathrm{H} 36$ & 119.4 \\
\hline $\mathrm{C} 26-\mathrm{C} 25-\mathrm{H} 25$ & 119.7 & $\mathrm{O} 32-\mathrm{C} 37-\mathrm{O} 31$ & $123.5(4)$ \\
\hline $\mathrm{C} 21-\mathrm{C} 26-\mathrm{C} 25$ & $122.3(10)$ & $\mathrm{O} 32-\mathrm{C} 37-\mathrm{C} 31$ & $117.7(4)$ \\
\hline $\mathrm{C} 21-\mathrm{C} 26-\mathrm{H} 26$ & 118.8 & $\mathrm{O} 31-\mathrm{C} 37-\mathrm{C} 31$ & $118.7(4)$ \\
\hline $\mathrm{C} 25-\mathrm{C} 26-\mathrm{H} 26$ & 118.8 & $\mathrm{H} 41-\mathrm{O} 41-\mathrm{H} 42$ & $105(3)$ \\
\hline $\mathrm{C} 24-\mathrm{O} 24-\mathrm{C} 27$ & $119.3(6)$ & & \\
\hline $\mathrm{C} 6-\mathrm{N} 1-\mathrm{C} 2-\mathrm{C} 3$ & $-56.4(3)$ & $\mathrm{C} 3-\mathrm{N} 4-\mathrm{C} 51-\mathrm{C} 52$ & $-37(18)$ \\
\hline $\mathrm{N} 1-\mathrm{C} 2-\mathrm{C} 3-\mathrm{N} 4$ & $55.4(4)$ & $\mathrm{C} 5-\mathrm{N} 4-\mathrm{C} 51-\mathrm{C} 52$ & $-168(11)$ \\
\hline $\mathrm{C} 2-\mathrm{C} 3-\mathrm{N} 4-\mathrm{C} 51$ & $175(9)$ & $\mathrm{C} 56-\mathrm{C} 51-\mathrm{C} 52-\mathrm{C} 53$ & $-9(19)$ \\
\hline $\mathrm{C} 2-\mathrm{C} 3-\mathrm{N} 4-\mathrm{C} 21$ & $173(3)$ & $\mathrm{N} 4-\mathrm{C} 51-\mathrm{C} 52-\mathrm{C} 53$ & $-178(9)$ \\
\hline $\mathrm{C} 2-\mathrm{C} 3-\mathrm{N} 4-\mathrm{C} 5$ & $-54.1(3)$ & $\mathrm{C} 51-\mathrm{C} 52-\mathrm{C} 53-\mathrm{C} 54$ & $7(11)$ \\
\hline $\mathrm{C} 51-\mathrm{N} 4-\mathrm{C} 5-\mathrm{C} 6$ & $-171(8)$ & $\mathrm{C} 52-\mathrm{C} 53-\mathrm{C} 54-\mathrm{C} 55$ & $-1(10)$ \\
\hline
\end{tabular}




$\begin{array}{ll}\mathrm{C} 21-\mathrm{N} 4-\mathrm{C} 5-\mathrm{C} 6 & -173(3) \\ \mathrm{C} 3-\mathrm{N} 4-\mathrm{C} 5-\mathrm{C} 6 & 54.8(3) \\ \mathrm{C} 2-\mathrm{N} 1-\mathrm{C} 6-\mathrm{C} 5 & 57.2(3) \\ \mathrm{N} 4-\mathrm{C} 5-\mathrm{C} 6-\mathrm{N} 1 & -56.8(4) \\ \mathrm{C} 3-\mathrm{N} 4-\mathrm{C} 21-\mathrm{C} 26 & 169(4) \\ \mathrm{C} 5-\mathrm{N} 4-\mathrm{C} 21-\mathrm{C} 26 & 38(7) \\ \mathrm{C} 3-\mathrm{N} 4-\mathrm{C} 21-\mathrm{C} 22 & -10(7) \\ \mathrm{C} 5-\mathrm{N} 4-\mathrm{C} 21-\mathrm{C} 22 & -140(4) \\ \mathrm{C} 26-\mathrm{C} 21-\mathrm{C} 22-\mathrm{C} 23 & 3(7) \\ \mathrm{N} 4-\mathrm{C} 21-\mathrm{C} 22-\mathrm{C} 23 & -178(4) \\ \mathrm{C} 21-\mathrm{C} 22-\mathrm{C} 23-\mathrm{C} 24 & -3(4) \\ \mathrm{C} 22-\mathrm{C} 23-\mathrm{C} 24-\mathrm{C} 25 & 1(3) \\ \mathrm{C} 22-\mathrm{C} 23-\mathrm{C} 24-\mathrm{O} 24 & -178.6(14) \\ \mathrm{C} 23-\mathrm{C} 24-\mathrm{C} 25-\mathrm{C} 26 & 1(5) \\ \mathrm{O} 24-\mathrm{C} 24-\mathrm{C} 25-\mathrm{C} 26 & -180(3) \\ \mathrm{C} 22-\mathrm{C} 21-\mathrm{C} 26-\mathrm{C} 25 & -2(7) \\ \mathrm{N} 4-\mathrm{C} 21-\mathrm{C} 26-\mathrm{C} 25 & 180(4) \\ \mathrm{C} 24-\mathrm{C} 25-\mathrm{C} 26-\mathrm{C} 21 & 0(6) \\ \mathrm{C} 25-\mathrm{C} 24-\mathrm{O} 24-\mathrm{C} 27 & -10(4) \\ \mathrm{C} 23-\mathrm{C} 24-\mathrm{O} 24-\mathrm{C} 27 & 169.9(15) \\ \mathrm{C} 3-\mathrm{N} 4-\mathrm{C} 51-\mathrm{C} 56 & 155(12) \\ \mathrm{C} 5-\mathrm{N} 4-\mathrm{C} 51-\mathrm{C} 56 & 24(19)\end{array}$

$-179(5)$
$-2(14)$
$176(8)$
$6(20)$
$174(13)$
$-1(17)$
$13(13)$
$-169(5)$
$0.8(5)$
$-179.2(3)$
$-0.3(6)$
$-0.4(6)$
$179.1(3)$
$0.5(6)$
$-179.0(3)$
$-0.7(5)$
$179.3(3)$
$0.1(5)$
$174.6(3)$
$-5.3(5)$
$-5.1(5)$
$174.9(3)$

Hydrogen-bond geometry $\left(\AA,{ }^{\circ}\right)$

\begin{tabular}{lllll}
\hline$D-\mathrm{H} \cdots A$ & $D-\mathrm{H}$ & $\mathrm{H} \cdots A$ & $D \cdots A$ & $D-\mathrm{H} \cdots A$ \\
\hline $\mathrm{N} 1-\mathrm{H} 11 \cdots \mathrm{O} 31$ & $1.09(3)$ & $1.71(3)$ & $2.790(4)$ & $176(3)$ \\
$\mathrm{N} 1-\mathrm{H} 12 \cdots \mathrm{O} 41$ & $0.83(3)$ & $1.98(3)$ & $2.811(4)$ & $174(3)$ \\
$\mathrm{O} 41-\mathrm{H} 41 \cdots \mathrm{O} 32^{\mathrm{i}}$ & $0.91(4)$ & $1.73(4)$ & $2.624(4)$ & $172(4)$ \\
$\mathrm{O} 41-\mathrm{H} 42 \cdots \mathrm{O} 31^{\mathrm{ii}}$ & $0.94(4)$ & $1.84(4)$ & $2.775(4)$ & $170(4)$ \\
$\mathrm{C} 2-\mathrm{H} 2 B \cdots \mathrm{O} 31^{\mathrm{iii}}$ & 0.97 & 2.52 & $3.467(4)$ & 165 \\
$\mathrm{C} 6-\mathrm{H} 6 B \cdots \mathrm{O} 41^{\mathrm{i}}$ & 0.97 & 2.60 & $3.408(4)$ & 141 \\
$\mathrm{C} 22-\mathrm{H} 22 \cdots \mathrm{Cg} 1^{\text {iv }}$ & 0.93 & 2.89 & $3.631(13)$ & 137 \\
$\mathrm{C} 26-\mathrm{H} 26 \cdots \mathrm{Cg} 1^{\text {iv }}$ & 0.93 & 2.81 & $3.58(2)$ & 141
\end{tabular}

Symmetry codes: (i) $-x+1,-y+1,-z+1$; (ii) $-x+2,-y+1,-z+1$; (iii) $-x+2,-y+2,-z+1$; (iv) $-x+1,-y+2,-z+1$.

4-(4-Methoxyphenyl)piperazin-1-ium 4-bromobenzoate monohydrate (IV)

Crystal data

$\mathrm{C}_{11} \mathrm{H}_{17} \mathrm{~N}_{2} \mathrm{O}^{+} \cdot \mathrm{C}_{7} \mathrm{H}_{4} \mathrm{BrO}_{2}^{-} \cdot \mathrm{H}_{2} \mathrm{O}$

$M_{r}=411.28$

Triclinic, $P \overline{1}$

$a=6.2004(8) \AA$

$b=7.4957$ (9) $\AA$

$c=20.440(2) \AA$

$\alpha=85.08(1)^{\circ}$

$\beta=87.37(1)^{\circ}$

$\gamma=85.00(1)^{\circ}$

$V=942.17(19) \AA^{3}$
$Z=2$

$F(000)=424$

$D_{\mathrm{x}}=1.450 \mathrm{Mg} \mathrm{m}^{-3}$

Mo $K \alpha$ radiation, $\lambda=0.71073 \AA$

Cell parameters from 3927 reflections

$\theta=2.8-27.9^{\circ}$

$\mu=2.21 \mathrm{~mm}^{-1}$

$T=293 \mathrm{~K}$

Plate, colourless

$0.48 \times 0.44 \times 0.16 \mathrm{~mm}$ 


\section{Data collection}

Oxford Diffraction Xcalibur with Sapphire CCD diffractometer

Radiation source: Enhance (Mo) X-ray Source Graphite monochromator

$\omega$ scans

Absorption correction: multi-scan

(CrysAlis RED; Oxford Diffraction, 2009)

$T_{\min }=0.536, T_{\max }=0.719$

\section{Refinement}

Refinement on $F^{2}$

Least-squares matrix: full

$R\left[F^{2}>2 \sigma\left(F^{2}\right)\right]=0.068$

$w R\left(F^{2}\right)=0.197$

$S=1.06$

3818 reflections

265 parameters

17 restraints
6176 measured reflections

3818 independent reflections

2063 reflections with $I>2 \sigma(I)$

$R_{\text {int }}=0.018$

$\theta_{\text {max }}=26.6^{\circ}, \theta_{\text {min }}=2.8^{\circ}$

$h=-7 \rightarrow 7$

$k=-8 \rightarrow 9$

$l=-23 \rightarrow 25$

Primary atom site location: difference Fourier map

Hydrogen site location: mixed

$\mathrm{H}$ atoms treated by a mixture of independent and constrained refinement

$w=1 /\left[\sigma^{2}\left(F_{\mathrm{o}}^{2}\right)+(0.0819 P)^{2}+0.9249 P\right]$

where $P=\left(F_{\mathrm{o}}^{2}+2 F_{\mathrm{c}}^{2}\right) / 3$

$(\Delta / \sigma)_{\max }=0.001$

$\Delta \rho_{\max }=0.94$ e $\AA^{-3}$

$\Delta \rho_{\min }=-0.64$ e $\AA^{-3}$

Special details

Experimental. Compound (IV). IR ( $\left.\mathrm{KBr}, \mathrm{cm}^{-1}\right) 3319(\mathrm{OH}), 3001\left(\mathrm{NH}_{2}\right), 2836\left(\mathrm{OCH}_{3}\right), 1580(\mathrm{COO}), 600(\mathrm{CBr})$. . NMR $\left.\left(\mathrm{CDCl}_{3}\right) \delta\left({ }^{1} \mathrm{H}\right)\right) 3.23\left(\mathrm{~m}, 4 \mathrm{H}\right.$, piperazine), $3.30\left(\mathrm{~m}, 4 \mathrm{H}\right.$, piperazine), $3.77\left(\mathrm{~s}, 3 \mathrm{H}, \mathrm{OCH}_{3}\right), 6.85$ (m, 4H, methoxyphenyl), 7.51 (d, J = 8.4 Hz, 2H, bromophenyl), 7.90 (d, J = 8.4 Hz,2H, bromophenyl).

Geometry. All esds (except the esd in the dihedral angle between two 1.s. planes) are estimated using the full covariance matrix. The cell esds are taken into account individually in the estimation of esds in distances, angles and torsion angles; correlations between esds in cell parameters are only used when they are defined by crystal symmetry. An approximate (isotropic) treatment of cell esds is used for estimating esds involving 1.s. planes.

Fractional atomic coordinates and isotropic or equivalent isotropic displacement parameters $\left(\AA^{2}\right)$

\begin{tabular}{|c|c|c|c|c|c|}
\hline & $x$ & $y$ & $z$ & $U_{\text {iso }} * / U_{\text {eq }}$ & Occ. $(<1)$ \\
\hline N1 & $0.7803(6)$ & $0.7425(5)$ & $0.45428(17)$ & $0.0475(9)$ & \\
\hline H11 & $0.813(7)$ & $0.770(6)$ & $0.488(2)$ & $0.057 *$ & \\
\hline H12 & $0.753(7)$ & $0.620(7)$ & $0.461(2)$ & $0.057^{*}$ & \\
\hline $\mathrm{C} 2$ & $0.9709(7)$ & $0.7508(6)$ & $0.4079(2)$ & $0.0530(11)$ & \\
\hline $\mathrm{H} 2 \mathrm{~A}$ & 1.0899 & 0.6702 & 0.4250 & $0.064 *$ & \\
\hline $\mathrm{H} 2 \mathrm{~B}$ & 1.0169 & 0.8718 & 0.4034 & $0.064 *$ & \\
\hline C3 & $0.9143(6)$ & $0.6978(6)$ & $0.3414(2)$ & $0.0502(10)$ & \\
\hline $\mathrm{H} 3 \mathrm{~A}$ & 1.0382 & 0.7086 & 0.3110 & $0.060^{*}$ & \\
\hline $\mathrm{H} 3 \mathrm{~B}$ & 0.8808 & 0.5732 & 0.3454 & $0.060^{*}$ & \\
\hline N4 & $0.7286(5)$ & $0.8112(4)$ & $0.31535(14)$ & $0.0389(7)$ & \\
\hline $\mathrm{C} 5$ & $0.5420(6)$ & $0.8089(6)$ & $0.36137(18)$ & $0.0454(10)$ & \\
\hline H5A & 0.4924 & 0.6891 & 0.3665 & $0.055^{*}$ & \\
\hline H5B & 0.4249 & 0.8909 & 0.3438 & $0.055^{*}$ & \\
\hline C6 & $0.5976(7)$ & $0.8631(6)$ & 0.42775 (19) & 0.0485 (10) & \\
\hline H6A & 0.6368 & 0.9862 & 0.4234 & $0.058 *$ & \\
\hline H6B & 0.4723 & 0.8565 & 0.4578 & $0.058 *$ & \\
\hline $\mathrm{C} 21$ & 0.6845 (19) & $0.784(6)$ & 0.2485 & $0.0421(11)$ & $0.80(2)$ \\
\hline
\end{tabular}




\begin{tabular}{|c|c|c|c|c|c|}
\hline $\mathrm{C} 22$ & $0.8313(11)$ & $0.6853(18)$ & 0.2086 & $0.066(3)$ & $0.80(2)$ \\
\hline $\mathrm{H} 22$ & 0.9604 & 0.6325 & 0.2256 & $0.079 *$ & $0.80(2)$ \\
\hline $\mathrm{C} 23$ & $0.7862(12)$ & $0.666(2)$ & $0.1442(3)$ & $0.084(4)$ & $0.80(2)$ \\
\hline $\mathrm{H} 23$ & 0.8895 & 0.6040 & 0.1183 & $0.101^{*}$ & $0.80(2)$ \\
\hline $\mathrm{C} 24$ & $0.5980(13)$ & $0.7331(16)$ & $0.1174(4)$ & $0.062(3)$ & $0.80(2)$ \\
\hline $\mathrm{C} 25$ & $0.4512(15)$ & $0.826(5)$ & $0.1561(6)$ & $0.080(4)$ & $0.80(2)$ \\
\hline $\mathrm{H} 25$ & 0.3201 & 0.8733 & 0.1390 & $0.096^{*}$ & $0.80(2)$ \\
\hline $\mathrm{C} 26$ & $0.4941(16)$ & $0.850(3)$ & $0.2209(4)$ & $0.064(4)$ & $0.80(2)$ \\
\hline $\mathrm{H} 26$ & 0.3899 & 0.9127 & 0.2462 & $0.077^{*}$ & $0.80(2)$ \\
\hline $\mathrm{O} 24$ & $0.5730(16)$ & $0.7011(17)$ & 0.0530 & $0.101(3)$ & $0.80(2)$ \\
\hline $\mathrm{C} 27$ & $0.373(2)$ & $0.743(3)$ & $0.0258(6)$ & $0.114(6)$ & $0.80(2)$ \\
\hline $\mathrm{H} 27 \mathrm{~A}$ & 0.2623 & 0.6966 & 0.0551 & $0.171^{*}$ & $0.80(2)$ \\
\hline H27B & 0.3717 & 0.6905 & -0.0154 & $0.171 *$ & $0.80(2)$ \\
\hline $\mathrm{H} 27 \mathrm{C}$ & 0.3464 & 0.8712 & 0.0186 & $0.171 *$ & $0.80(2)$ \\
\hline C51 & $0.672(7)$ & $0.79(2)$ & $0.2512(15)$ & $0.0421(11)$ & $0.20(2)$ \\
\hline C52 & $0.838(5)$ & $0.786(6)$ & $0.2026(13)$ & $0.066(3)$ & $0.20(2)$ \\
\hline H52 & 0.9785 & 0.8027 & 0.2134 & $0.079 *$ & $0.20(2)$ \\
\hline C53 & $0.795(5)$ & $0.760(7)$ & $0.1384(13)$ & $0.084(4)$ & $0.20(2)$ \\
\hline H53 & 0.9078 & 0.7242 & 0.1096 & $0.101^{*}$ & $0.20(2)$ \\
\hline C54 & $0.590(5)$ & $0.787(9)$ & 0.1169 (14) & $0.062(3)$ & $0.20(2)$ \\
\hline C55 & $0.426(6)$ & $0.81(2)$ & $0.163(2)$ & $0.080(4)$ & $0.20(2)$ \\
\hline H55 & 0.2833 & 0.8234 & 0.1492 & $0.096^{*}$ & $0.20(2)$ \\
\hline C56 & $0.466(6)$ & $0.818(13)$ & $0.2286(19)$ & $0.064(4)$ & $0.20(2)$ \\
\hline H56 & 0.3515 & 0.8441 & 0.2581 & $0.077^{*}$ & $0.20(2)$ \\
\hline O54 & $0.565(7)$ & $0.773(7)$ & $0.0512(15)$ & $0.101(3)$ & $0.20(2)$ \\
\hline C57 & $0.364(9)$ & $0.817(13)$ & $0.025(3)$ & $0.114(6)$ & $0.20(2)$ \\
\hline H57A & 0.2541 & 0.7740 & 0.0554 & $0.171^{*}$ & $0.20(2)$ \\
\hline H57B & 0.3582 & 0.7635 & -0.0157 & $0.171^{*}$ & $0.20(2)$ \\
\hline $\mathrm{H} 57 \mathrm{C}$ & 0.3404 & 0.9457 & 0.0173 & $0.171 *$ & $0.20(2)$ \\
\hline C31 & $0.8064(6)$ & $0.7392(5)$ & $0.6932(2)$ & $0.0436(9)$ & \\
\hline C32 & $0.6590(8)$ & $0.6690(7)$ & $0.7386(3)$ & $0.0661(13)$ & \\
\hline H32 & 0.5388 & 0.6192 & 0.7245 & $0.079 *$ & \\
\hline C33 & $0.6890(10)$ & $0.6724(8)$ & 0.8053 & $0.0832(17)$ & \\
\hline H33 & 0.5888 & 0.6259 & 0.8360 & $0.100^{*}$ & \\
\hline C34 & $0.8682(10)$ & $0.7450(8)$ & $0.8256(2)$ & $0.0802(16)$ & \\
\hline Br34 & $0.90280(17)$ & $0.75220(15)$ & $0.91705(3)$ & $0.1491(5)$ & \\
\hline $\mathrm{C} 35$ & $1.0170(9)$ & $0.8124(7)$ & $0.7814(2)$ & $0.0683(14)$ & \\
\hline H35 & 1.1383 & 0.8599 & 0.7959 & $0.082 *$ & \\
\hline $\mathrm{C} 36$ & $0.9870(7)$ & $0.8100(6)$ & $0.7146(2)$ & $0.0500(10)$ & \\
\hline H36 & 1.0884 & 0.8561 & 0.6843 & $0.060 *$ & \\
\hline C37 & $0.7721(7)$ & $0.7357(6)$ & $0.6201(2)$ & $0.0528(11)$ & \\
\hline $\mathrm{O} 31$ & $0.8989(6)$ & $0.8126(4)$ & $0.57976(15)$ & $0.0631(9)$ & \\
\hline $\mathrm{O} 32$ & $0.6180(7)$ & $0.6578(6)$ & $0.6044(2)$ & $0.0999(14)$ & \\
\hline O41 & $0.7233(6)$ & $0.3743(5)$ & $0.46351(17)$ & $0.0633(9)$ & \\
\hline H41 & $0.620(10)$ & $0.353(8)$ & $0.445(3)$ & $0.095^{*}$ & \\
\hline $\mathrm{H} 42$ & $0.820(11)$ & $0.313(9)$ & 0.449 (3) & $0.095^{*}$ & \\
\hline
\end{tabular}


Atomic displacement parameters $\left(\AA^{2}\right)$

\begin{tabular}{|c|c|c|c|c|c|c|}
\hline & $U^{11}$ & $U^{22}$ & $U^{33}$ & $U^{12}$ & $U^{13}$ & $U^{23}$ \\
\hline N1 & $0.062(2)$ & $0.048(2)$ & $0.0349(17)$ & $-0.0089(18)$ & $-0.0128(16)$ & $-0.0048(16)$ \\
\hline $\mathrm{C} 2$ & $0.044(2)$ & $0.067(3)$ & $0.049(2)$ & $-0.004(2)$ & $-0.0092(19)$ & $-0.004(2)$ \\
\hline $\mathrm{C} 3$ & $0.037(2)$ & $0.067(3)$ & $0.046(2)$ & $0.002(2)$ & $-0.0018(18)$ & $-0.003(2)$ \\
\hline N4 & 0.0365 (17) & $0.047(2)$ & $0.0333(15)$ & $-0.0045(14)$ & $0.0000(13)$ & $-0.0017(14)$ \\
\hline $\mathrm{C} 5$ & $0.039(2)$ & $0.059(3)$ & $0.038(2)$ & 0.0005 (19) & $-0.0004(17)$ & $-0.0020(19)$ \\
\hline C6 & $0.055(3)$ & $0.052(3)$ & $0.037(2)$ & $0.003(2)$ & $0.0016(18)$ & $-0.0034(18)$ \\
\hline $\mathrm{C} 21$ & $0.044(3)$ & $0.048(3)$ & $0.034(2)$ & $-0.006(3)$ & $0.0000(19)$ & $-0.002(3)$ \\
\hline $\mathrm{C} 22$ & $0.053(3)$ & $0.093(9)$ & $0.048(3)$ & $0.025(4)$ & $-0.009(2)$ & $-0.017(4)$ \\
\hline $\mathrm{C} 23$ & $0.076(4)$ & $0.122(10)$ & 0.049 (3) & $0.036(5)$ & 0.001 & $-0.031(5)$ \\
\hline $\mathrm{C} 24$ & $0.072(3)$ & $0.079(9)$ & $0.036(2)$ & $0.009(4)$ & $-0.007(2)$ & $-0.018(3)$ \\
\hline $\mathrm{C} 25$ & $0.058(4)$ & $0.133(10)$ & $0.046(4)$ & $0.015(7)$ & -0.015 & $-0.008(6)$ \\
\hline $\mathrm{C} 26$ & $0.055(4)$ & $0.099(10)$ & $0.036(3)$ & $0.015(5)$ & $-0.001(2)$ & $-0.015(5)$ \\
\hline $\mathrm{O} 24$ & $0.108(3)$ & $0.148(10)$ & $0.046(2)$ & $0.033(5)$ & $-0.019(2)$ & $-0.037(4)$ \\
\hline $\mathrm{C} 27$ & $0.108(5)$ & 0.181 (19) & $0.058(4)$ & $0.008(7)$ & -0.030 & $-0.038(7)$ \\
\hline C51 & $0.044(3)$ & $0.048(3)$ & $0.034(2)$ & $-0.006(3)$ & 0.0000 (19) & $-0.002(3)$ \\
\hline C52 & $0.053(3)$ & $0.093(9)$ & $0.048(3)$ & $0.025(4)$ & -0.009 (2) & -0.017 (4) \\
\hline $\mathrm{C} 53$ & $0.076(4)$ & $0.122(10)$ & $0.049(3)$ & $0.036(5)$ & $0.001(3)$ & $-0.031(5)$ \\
\hline C54 & $0.072(3)$ & $0.079(9)$ & $0.036(2)$ & 0.009 (4) & $-0.007(2)$ & -0.018 \\
\hline C55 & $0.058(4)$ & $0.133(10)$ & $0.046(4)$ & $0.015(7)$ & $-0.015(3)$ & $-0.008(6)$ \\
\hline C56 & $0.055(4)$ & $0.099(10)$ & $0.036(3)$ & $0.015(5)$ & $-0.001(2)$ & $-0.015(5)$ \\
\hline O54 & $0.108(3)$ & $0.148(10)$ & $0.046(2)$ & $0.033(5)$ & $-0.019(2)$ & $-0.037(4)$ \\
\hline $\mathrm{C} 57$ & $0.108(5)$ & $0.181(19)$ & $0.058(4)$ & $0.008(7)$ & $-0.030(4)$ & $-0.038(7)$ \\
\hline $\mathrm{C} 31$ & $0.041(2)$ & $0.040(2)$ & $0.049(2)$ & $0.0019(18)$ & $-0.0051(18)$ & $-0.0056(18)$ \\
\hline $\mathrm{C} 32$ & $0.052(3)$ & $0.070(3)$ & $0.076(3)$ & $-0.009(2)$ & $0.001(2)$ & -0.005 \\
\hline $\mathrm{C} 33$ & $0.082(4)$ & $0.098(4)$ & $0.063(3)$ & $-0.002(3)$ & $0.023(3)$ & $0.011(3)$ \\
\hline C34 & $0.089(4)$ & $0.100(4)$ & $0.048(3)$ & $0.001(3)$ & $-0.006(3)$ & $0.004(3)$ \\
\hline Br34 & $0.1950(10)$ & $0.2001(11)$ & $0.0444(4)$ & $0.0349(8)$ & $-0.0150(4)$ & $-0.0114(4)$ \\
\hline $\mathrm{C} 35$ & $0.073(3)$ & $0.082(4)$ & $0.053(3)$ & $-0.006(3)$ & -0.021 & -0.010 \\
\hline C36 & $0.049(2)$ & $0.056(3)$ & $0.046(2)$ & -0.010 & -0.0083 (19) & $0.000(2)$ \\
\hline C37 & $0.051(3)$ & $0.045(3)$ & $0.063(3)$ & $0.006(2)$ & -0.020 & $-0.012(2)$ \\
\hline $\mathrm{O} 31$ & $0.083(2)$ & $0.063(2)$ & $0.0446(17)$ & $-0.0054(18)$ & $-0.0124(17)$ & $-0.0053(15)$ \\
\hline $\mathrm{O} 32$ & $0.087(3)$ & $0.130(4)$ & $0.094(3)$ & $-0.037(3)$ & $-0.038(2)$ & $-0.024(3)$ \\
\hline $\mathrm{O} 41$ & $0.064(2)$ & $0.065(2)$ & $0.064(2)$ & $-0.0133(17)$ & $-0.0210(17)$ & $-0.0029(17)$ \\
\hline
\end{tabular}

Geometric parameters $\left(\AA,{ }^{\circ}\right)$

\begin{tabular}{llll}
\hline $\mathrm{N} 1-\mathrm{C} 6$ & $1.479(5)$ & $\mathrm{C} 27-\mathrm{H} 27 \mathrm{C}$ & 0.9600 \\
$\mathrm{~N} 1-\mathrm{C} 2$ & $1.483(6)$ & $\mathrm{C} 51-\mathrm{C} 56$ & $1.369(11)$ \\
$\mathrm{N} 1-\mathrm{H} 11$ & $0.78(5)$ & $\mathrm{C} 51-\mathrm{C} 52$ & $1.40(2)$ \\
$\mathrm{N} 1-\mathrm{H} 12$ & $0.95(5)$ & $\mathrm{C} 52-\mathrm{C} 53$ & 0.9300 \\
$\mathrm{C} 2-\mathrm{C} 3$ & $1.513(6)$ & $\mathrm{C} 52-\mathrm{H} 52$ & $1.353(11)$ \\
$\mathrm{C} 2-\mathrm{H} 2 \mathrm{~A}$ & 0.9700 & $\mathrm{C} 53-\mathrm{C} 54$ & 0.9300 \\
$\mathrm{C} 2-\mathrm{H} 2 \mathrm{~B}$ & 0.9700 & $\mathrm{C} 53-\mathrm{H} 53$ & $1.362(19)$ \\
$\mathrm{C} 3-\mathrm{N} 4$ & $1.463(5)$ & $\mathrm{C} 54-\mathrm{C} 55$ & $1.374(10)$ \\
$\mathrm{C} 3-\mathrm{H} 3 \mathrm{~A}$ & 0.9700 & $\mathrm{C} 54-\mathrm{O} 54$ &
\end{tabular}




\begin{tabular}{|c|c|c|c|}
\hline $\mathrm{C} 3-\mathrm{H} 3 \mathrm{~B}$ & 0.9700 & $\mathrm{C} 55-\mathrm{C} 56$ & $1.392(11)$ \\
\hline N4-C51 & $1.40(4)$ & $\mathrm{C} 55-\mathrm{H} 55$ & 0.9300 \\
\hline $\mathrm{N} 4-\mathrm{C} 21$ & $1.441(10)$ & C56-H56 & 0.9300 \\
\hline $\mathrm{N} 4-\mathrm{C} 5$ & $1.458(5)$ & $\mathrm{O} 54-\mathrm{C} 57$ & $1.383(12)$ \\
\hline $\mathrm{C} 5-\mathrm{C} 6$ & $1.512(5)$ & C57-H57A & 0.9600 \\
\hline $\mathrm{C} 5-\mathrm{H} 5 \mathrm{~A}$ & 0.9700 & C57-H57B & 0.9600 \\
\hline C5-H5B & 0.9700 & C57-H57C & 0.9600 \\
\hline C6-H6A & 0.9700 & $\mathrm{C} 31-\mathrm{C} 32$ & $1.377(6)$ \\
\hline C6-H6B & 0.9700 & $\mathrm{C} 31-\mathrm{C} 36$ & $1.385(5)$ \\
\hline $\mathrm{C} 21-\mathrm{C} 26$ & $1.367(10)$ & $\mathrm{C} 31-\mathrm{C} 37$ & $1.521(6)$ \\
\hline $\mathrm{C} 21-\mathrm{C} 22$ & $1.399(19)$ & $\mathrm{C} 32-\mathrm{C} 33$ & $1.387(7)$ \\
\hline $\mathrm{C} 22-\mathrm{C} 23$ & $1.380(7)$ & $\mathrm{C} 32-\mathrm{H} 32$ & 0.9300 \\
\hline $\mathrm{C} 22-\mathrm{H} 22$ & 0.9300 & $\mathrm{C} 33-\mathrm{C} 34$ & $1.375(8)$ \\
\hline $\mathrm{C} 23-\mathrm{C} 24$ & $1.351(8)$ & $\mathrm{C} 33-\mathrm{H} 33$ & 0.9300 \\
\hline $\mathrm{C} 23-\mathrm{H} 23$ & 0.9300 & $\mathrm{C} 34-\mathrm{C} 35$ & $1.360(8)$ \\
\hline $\mathrm{C} 24-\mathrm{C} 25$ & $1.362(16)$ & $\mathrm{C} 34-\mathrm{Br} 34$ & $1.897(5)$ \\
\hline $\mathrm{C} 24-\mathrm{O} 24$ & $1.374(6)$ & $\mathrm{C} 35-\mathrm{C} 36$ & $1.388(6)$ \\
\hline $\mathrm{C} 25-\mathrm{C} 26$ & $1.392(8)$ & $\mathrm{C} 35-\mathrm{H} 35$ & 0.9300 \\
\hline $\mathrm{C} 25-\mathrm{H} 25$ & 0.9300 & C36-H36 & 0.9300 \\
\hline $\mathrm{C} 26-\mathrm{H} 26$ & 0.9300 & $\mathrm{C} 37-\mathrm{O} 32$ & $1.231(5)$ \\
\hline $\mathrm{O} 24-\mathrm{C} 27$ & $1.383(8)$ & $\mathrm{C} 37-\mathrm{O} 31$ & $1.252(6)$ \\
\hline $\mathrm{C} 27-\mathrm{H} 27 \mathrm{~A}$ & 0.9600 & $\mathrm{O} 41-\mathrm{H} 41$ & $0.79(6)$ \\
\hline $\mathrm{C} 27-\mathrm{H} 27 \mathrm{~B}$ & 0.9600 & $\mathrm{O} 41-\mathrm{H} 42$ & $0.79(7)$ \\
\hline $\mathrm{C} 6-\mathrm{N} 1-\mathrm{C} 2$ & $109.7(3)$ & $\mathrm{O} 24-\mathrm{C} 27-\mathrm{H} 27 \mathrm{~A}$ & 109.5 \\
\hline $\mathrm{C} 6-\mathrm{N} 1-\mathrm{H} 11$ & $111(3)$ & $\mathrm{O} 24-\mathrm{C} 27-\mathrm{H} 27 \mathrm{~B}$ & 109.5 \\
\hline $\mathrm{C} 2-\mathrm{N} 1-\mathrm{H} 11$ & $108(3)$ & $\mathrm{H} 27 \mathrm{~A}-\mathrm{C} 27-\mathrm{H} 27 \mathrm{~B}$ & 109.5 \\
\hline $\mathrm{C} 6-\mathrm{N} 1-\mathrm{H} 12$ & $115(3)$ & $\mathrm{O} 24-\mathrm{C} 27-\mathrm{H} 27 \mathrm{C}$ & 109.5 \\
\hline $\mathrm{C} 2-\mathrm{N} 1-\mathrm{H} 12$ & $106(3)$ & $\mathrm{H} 27 \mathrm{~A}-\mathrm{C} 27-\mathrm{H} 27 \mathrm{C}$ & 109.5 \\
\hline $\mathrm{H} 11-\mathrm{N} 1-\mathrm{H} 12$ & $106(4)$ & $\mathrm{H} 27 \mathrm{~B}-\mathrm{C} 27-\mathrm{H} 27 \mathrm{C}$ & 109.5 \\
\hline $\mathrm{N} 1-\mathrm{C} 2-\mathrm{C} 3$ & $110.1(3)$ & $\mathrm{C} 56-\mathrm{C} 51-\mathrm{C} 52$ & $115(2)$ \\
\hline $\mathrm{N} 1-\mathrm{C} 2-\mathrm{H} 2 \mathrm{~A}$ & 109.6 & $\mathrm{C} 56-\mathrm{C} 51-\mathrm{N} 4$ & $125(3)$ \\
\hline $\mathrm{C} 3-\mathrm{C} 2-\mathrm{H} 2 \mathrm{~A}$ & 109.6 & $\mathrm{C} 52-\mathrm{C} 51-\mathrm{N} 4$ & $117(4)$ \\
\hline $\mathrm{N} 1-\mathrm{C} 2-\mathrm{H} 2 \mathrm{~B}$ & 109.6 & $\mathrm{C} 53-\mathrm{C} 52-\mathrm{C} 51$ & 120.4 (19) \\
\hline $\mathrm{C} 3-\mathrm{C} 2-\mathrm{H} 2 \mathrm{~B}$ & 109.6 & $\mathrm{C} 53-\mathrm{C} 52-\mathrm{H} 52$ & 119.8 \\
\hline $\mathrm{H} 2 \mathrm{~A}-\mathrm{C} 2-\mathrm{H} 2 \mathrm{~B}$ & 108.1 & $\mathrm{C} 51-\mathrm{C} 52-\mathrm{H} 52$ & 119.8 \\
\hline $\mathrm{N} 4-\mathrm{C} 3-\mathrm{C} 2$ & $111.3(3)$ & $\mathrm{C} 54-\mathrm{C} 53-\mathrm{C} 52$ & $120.8(13)$ \\
\hline $\mathrm{N} 4-\mathrm{C} 3-\mathrm{H} 3 \mathrm{~A}$ & 109.4 & $\mathrm{C} 54-\mathrm{C} 53-\mathrm{H} 53$ & 119.6 \\
\hline $\mathrm{C} 2-\mathrm{C} 3-\mathrm{H} 3 \mathrm{~A}$ & 109.4 & $\mathrm{C} 52-\mathrm{C} 53-\mathrm{H} 53$ & 119.6 \\
\hline $\mathrm{N} 4-\mathrm{C} 3-\mathrm{H} 3 \mathrm{~B}$ & 109.4 & $\mathrm{C} 53-\mathrm{C} 54-\mathrm{C} 55$ & $117.8(11)$ \\
\hline $\mathrm{C} 2-\mathrm{C} 3-\mathrm{H} 3 \mathrm{~B}$ & 109.4 & $\mathrm{C} 53-\mathrm{C} 54-\mathrm{O} 54$ & $116.7(13)$ \\
\hline $\mathrm{H} 3 \mathrm{~A}-\mathrm{C} 3-\mathrm{H} 3 \mathrm{~B}$ & 108.0 & $\mathrm{C} 55-\mathrm{C} 54-\mathrm{O} 54$ & $125.2(15)$ \\
\hline $\mathrm{C} 51-\mathrm{N} 4-\mathrm{C} 5$ & $112(2)$ & $\mathrm{C} 54-\mathrm{C} 55-\mathrm{C} 56$ & $121.2(16)$ \\
\hline $\mathrm{C} 21-\mathrm{N} 4-\mathrm{C} 5$ & $115.3(7)$ & C54-C55-H55 & 119.4 \\
\hline $\mathrm{C} 51-\mathrm{N} 4-\mathrm{C} 3$ & $117(4)$ & $\mathrm{C} 56-\mathrm{C} 55-\mathrm{H} 55$ & 119.4 \\
\hline $\mathrm{C} 21-\mathrm{N} 4-\mathrm{C} 3$ & $114.0(11)$ & $\mathrm{C} 51-\mathrm{C} 56-\mathrm{C} 55$ & $121.4(12)$ \\
\hline $\mathrm{C} 5-\mathrm{N} 4-\mathrm{C} 3$ & $111.5(3)$ & $\mathrm{C} 51-\mathrm{C} 56-\mathrm{H} 56$ & 119.3 \\
\hline $\mathrm{N} 4-\mathrm{C} 5-\mathrm{C} 6$ & $111.4(3)$ & $\mathrm{C} 55-\mathrm{C} 56-\mathrm{H} 56$ & 119.3 \\
\hline
\end{tabular}




\begin{tabular}{|c|c|c|c|}
\hline $\mathrm{N} 4-\mathrm{C} 5-\mathrm{H} 5 \mathrm{~A}$ & 109.4 & $\mathrm{C} 54-\mathrm{O} 54-\mathrm{C} 57$ & $119.3(17)$ \\
\hline $\mathrm{C} 6-\mathrm{C} 5-\mathrm{H} 5 \mathrm{~A}$ & 109.4 & $\mathrm{O} 54-\mathrm{C} 57-\mathrm{H} 57 \mathrm{~A}$ & 109.5 \\
\hline $\mathrm{N} 4-\mathrm{C} 5-\mathrm{H} 5 \mathrm{~B}$ & 109.4 & $\mathrm{O} 54-\mathrm{C} 57-\mathrm{H} 57 \mathrm{~B}$ & 109.5 \\
\hline $\mathrm{C} 6-\mathrm{C} 5-\mathrm{H} 5 \mathrm{~B}$ & 109.4 & $\mathrm{H} 57 \mathrm{~A}-\mathrm{C} 57-\mathrm{H} 57 \mathrm{~B}$ & 109.5 \\
\hline $\mathrm{H} 5 \mathrm{~A}-\mathrm{C} 5-\mathrm{H} 5 \mathrm{~B}$ & 108.0 & $\mathrm{O} 54-\mathrm{C} 57-\mathrm{H} 57 \mathrm{C}$ & 109.5 \\
\hline $\mathrm{N} 1-\mathrm{C} 6-\mathrm{C} 5$ & $110.0(3)$ & $\mathrm{H} 57 \mathrm{~A}-\mathrm{C} 57-\mathrm{H} 57 \mathrm{C}$ & 109.5 \\
\hline $\mathrm{N} 1-\mathrm{C} 6-\mathrm{H} 6 \mathrm{~A}$ & 109.7 & $\mathrm{H} 57 \mathrm{~B}-\mathrm{C} 57-\mathrm{H} 57 \mathrm{C}$ & 109.5 \\
\hline $\mathrm{C} 5-\mathrm{C} 6-\mathrm{H} 6 \mathrm{~A}$ & 109.7 & $\mathrm{C} 32-\mathrm{C} 31-\mathrm{C} 36$ & $119.5(4)$ \\
\hline $\mathrm{N} 1-\mathrm{C} 6-\mathrm{H} 6 \mathrm{~B}$ & 109.7 & $\mathrm{C} 32-\mathrm{C} 31-\mathrm{C} 37$ & $120.0(4)$ \\
\hline $\mathrm{C} 5-\mathrm{C} 6-\mathrm{H} 6 \mathrm{~B}$ & 109.7 & $\mathrm{C} 36-\mathrm{C} 31-\mathrm{C} 37$ & $120.5(4)$ \\
\hline $\mathrm{H} 6 \mathrm{~A}-\mathrm{C} 6-\mathrm{H} 6 \mathrm{~B}$ & 108.2 & $\mathrm{C} 31-\mathrm{C} 32-\mathrm{C} 33$ & $120.3(5)$ \\
\hline $\mathrm{C} 26-\mathrm{C} 21-\mathrm{C} 22$ & $116.1(7)$ & $\mathrm{C} 31-\mathrm{C} 32-\mathrm{H} 32$ & 119.9 \\
\hline $\mathrm{C} 26-\mathrm{C} 21-\mathrm{N} 4$ & $121.7(13)$ & $\mathrm{C} 33-\mathrm{C} 32-\mathrm{H} 32$ & 119.9 \\
\hline $\mathrm{C} 22-\mathrm{C} 21-\mathrm{N} 4$ & $122.2(10)$ & $\mathrm{C} 34-\mathrm{C} 33-\mathrm{C} 32$ & $119.4(5)$ \\
\hline $\mathrm{C} 23-\mathrm{C} 22-\mathrm{C} 21$ & $120.6(6)$ & $\mathrm{C} 34-\mathrm{C} 33-\mathrm{H} 33$ & 120.3 \\
\hline $\mathrm{C} 23-\mathrm{C} 22-\mathrm{H} 22$ & 119.7 & $\mathrm{C} 32-\mathrm{C} 33-\mathrm{H} 33$ & 120.3 \\
\hline $\mathrm{C} 21-\mathrm{C} 22-\mathrm{H} 22$ & 119.7 & $\mathrm{C} 35-\mathrm{C} 34-\mathrm{C} 33$ & $121.1(5)$ \\
\hline $\mathrm{C} 24-\mathrm{C} 23-\mathrm{C} 22$ & $122.6(5)$ & $\mathrm{C} 35-\mathrm{C} 34-\mathrm{Br} 34$ & $120.3(5)$ \\
\hline $\mathrm{C} 24-\mathrm{C} 23-\mathrm{H} 23$ & 118.7 & $\mathrm{C} 33-\mathrm{C} 34-\mathrm{Br} 34$ & $118.6(4)$ \\
\hline $\mathrm{C} 22-\mathrm{C} 23-\mathrm{H} 23$ & 118.7 & $\mathrm{C} 34-\mathrm{C} 35-\mathrm{C} 36$ & $119.7(5)$ \\
\hline $\mathrm{C} 23-\mathrm{C} 24-\mathrm{C} 25$ & $117.4(5)$ & $\mathrm{C} 34-\mathrm{C} 35-\mathrm{H} 35$ & 120.2 \\
\hline $\mathrm{C} 23-\mathrm{C} 24-\mathrm{O} 24$ & $116.5(5)$ & $\mathrm{C} 36-\mathrm{C} 35-\mathrm{H} 35$ & 120.2 \\
\hline $\mathrm{C} 25-\mathrm{C} 24-\mathrm{O} 24$ & $126.1(6)$ & $\mathrm{C} 31-\mathrm{C} 36-\mathrm{C} 35$ & $120.1(4)$ \\
\hline $\mathrm{C} 24-\mathrm{C} 25-\mathrm{C} 26$ & $121.1(10)$ & $\mathrm{C} 31-\mathrm{C} 36-\mathrm{H} 36$ & 119.9 \\
\hline $\mathrm{C} 24-\mathrm{C} 25-\mathrm{H} 25$ & 119.5 & $\mathrm{C} 35-\mathrm{C} 36-\mathrm{H} 36$ & 119.9 \\
\hline $\mathrm{C} 26-\mathrm{C} 25-\mathrm{H} 25$ & 119.5 & $\mathrm{O} 32-\mathrm{C} 37-\mathrm{O} 31$ & $124.0(5)$ \\
\hline $\mathrm{C} 21-\mathrm{C} 26-\mathrm{C} 25$ & $122.1(10)$ & $\mathrm{O} 32-\mathrm{C} 37-\mathrm{C} 31$ & $117.1(5)$ \\
\hline $\mathrm{C} 21-\mathrm{C} 26-\mathrm{H} 26$ & 119.0 & $\mathrm{O} 31-\mathrm{C} 37-\mathrm{C} 31$ & $118.9(4)$ \\
\hline $\mathrm{C} 25-\mathrm{C} 26-\mathrm{H} 26$ & 119.0 & $\mathrm{H} 41-\mathrm{O} 41-\mathrm{H} 42$ & $105(6)$ \\
\hline $\mathrm{C} 24-\mathrm{O} 24-\mathrm{C} 27$ & $119.2(5)$ & & \\
\hline $\mathrm{C} 6-\mathrm{N} 1-\mathrm{C} 2-\mathrm{C} 3$ & $-58.3(5)$ & $\mathrm{C} 5-\mathrm{N} 4-\mathrm{C} 51-\mathrm{C} 52$ & $-180(9)$ \\
\hline $\mathrm{N} 1-\mathrm{C} 2-\mathrm{C} 3-\mathrm{N} 4$ & $56.7(5)$ & $\mathrm{C} 3-\mathrm{N} 4-\mathrm{C} 51-\mathrm{C} 52$ & $-49(14)$ \\
\hline $\mathrm{C} 2-\mathrm{C} 3-\mathrm{N} 4-\mathrm{C} 51$ & $174(7)$ & $\mathrm{C} 56-\mathrm{C} 51-\mathrm{C} 52-\mathrm{C} 53$ & $-20(15)$ \\
\hline $\mathrm{C} 2-\mathrm{C} 3-\mathrm{N} 4-\mathrm{C} 21$ & $172.1(16)$ & $\mathrm{N} 4-\mathrm{C} 51-\mathrm{C} 52-\mathrm{C} 53$ & $179(8)$ \\
\hline $\mathrm{C} 2-\mathrm{C} 3-\mathrm{N} 4-\mathrm{C} 5$ & $-55.2(4)$ & $\mathrm{C} 51-\mathrm{C} 52-\mathrm{C} 53-\mathrm{C} 54$ & $19(9)$ \\
\hline $\mathrm{C} 51-\mathrm{N} 4-\mathrm{C} 5-\mathrm{C} 6$ & $-171(8)$ & $\mathrm{C} 52-\mathrm{C} 53-\mathrm{C} 54-\mathrm{C} 55$ & $-10(12)$ \\
\hline $\mathrm{C} 21-\mathrm{N} 4-\mathrm{C} 5-\mathrm{C} 6$ & $-172.5(19)$ & $\mathrm{C} 52-\mathrm{C} 53-\mathrm{C} 54-\mathrm{O} 54$ & $175(5)$ \\
\hline $\mathrm{C} 3-\mathrm{N} 4-\mathrm{C} 5-\mathrm{C} 6$ & $55.6(4)$ & $\mathrm{C} 53-\mathrm{C} 54-\mathrm{C} 55-\mathrm{C} 56$ & $3(19)$ \\
\hline $\mathrm{C} 2-\mathrm{N} 1-\mathrm{C} 6-\mathrm{C} 5$ & $58.6(4)$ & $\mathrm{O} 54-\mathrm{C} 54-\mathrm{C} 55-\mathrm{C} 56$ & $178(10)$ \\
\hline $\mathrm{N} 4-\mathrm{C} 5-\mathrm{C} 6-\mathrm{N} 1$ & $-57.4(4)$ & $\mathrm{C} 52-\mathrm{C} 51-\mathrm{C} 56-\mathrm{C} 55$ & $13(18)$ \\
\hline $\mathrm{C} 5-\mathrm{N} 4-\mathrm{C} 21-\mathrm{C} 26$ & $36(4)$ & $\mathrm{N} 4-\mathrm{C} 51-\mathrm{C} 56-\mathrm{C} 55$ & $173(14)$ \\
\hline $\mathrm{C} 3-\mathrm{N} 4-\mathrm{C} 21-\mathrm{C} 26$ & $166(2)$ & $\mathrm{C} 54-\mathrm{C} 55-\mathrm{C} 56-\mathrm{C} 51$ & $-5(21)$ \\
\hline $\mathrm{C} 5-\mathrm{N} 4-\mathrm{C} 21-\mathrm{C} 22$ & $-143(2)$ & $\mathrm{C} 53-\mathrm{C} 54-\mathrm{O} 54-\mathrm{C} 57$ & $-173(6)$ \\
\hline $\mathrm{C} 3-\mathrm{N} 4-\mathrm{C} 21-\mathrm{C} 22$ & $-12(4)$ & $\mathrm{C} 55-\mathrm{C} 54-\mathrm{O} 54-\mathrm{C} 57$ & $12(14)$ \\
\hline $\mathrm{C} 26-\mathrm{C} 21-\mathrm{C} 22-\mathrm{C} 23$ & $3(4)$ & $\mathrm{C} 36-\mathrm{C} 31-\mathrm{C} 32-\mathrm{C} 33$ & $1.2(7)$ \\
\hline $\mathrm{N} 4-\mathrm{C} 21-\mathrm{C} 22-\mathrm{C} 23$ & $-178(2)$ & $\mathrm{C} 37-\mathrm{C} 31-\mathrm{C} 32-\mathrm{C} 33$ & $-179.8(5)$ \\
\hline $\mathrm{C} 21-\mathrm{C} 22-\mathrm{C} 23-\mathrm{C} 24$ & $-3(2)$ & $\mathrm{C} 31-\mathrm{C} 32-\mathrm{C} 33-\mathrm{C} 34$ & $-0.5(8)$ \\
\hline
\end{tabular}




$\begin{array}{llll}\mathrm{C} 22-\mathrm{C} 23-\mathrm{C} 24-\mathrm{C} 25 & 1(2) & \mathrm{C} 32-\mathrm{C} 33-\mathrm{C} 34-\mathrm{C} 35 & -0.4(9) \\ \mathrm{C} 22-\mathrm{C} 23-\mathrm{C} 24-\mathrm{O} 24 & -178.9(9) & \mathrm{C} 32-\mathrm{C} 33-\mathrm{C} 34-\mathrm{Br} 34 & 178.8(4) \\ \mathrm{C} 23-\mathrm{C} 24-\mathrm{C} 25-\mathrm{C} 26 & 0(4) & \mathrm{C} 33-\mathrm{C} 34-\mathrm{C} 35-\mathrm{C} 36 & 0.7(9) \\ \mathrm{O} 24-\mathrm{C} 24-\mathrm{C} 25-\mathrm{C} 26 & 180(2) & \mathrm{B} 34-\mathrm{C} 34-\mathrm{C} 35-\mathrm{C} 36 & -178.5(4) \\ \mathrm{C} 22-\mathrm{C} 21-\mathrm{C} 26-\mathrm{C} 25 & -2(4) & \mathrm{C} 32-\mathrm{C} 31-\mathrm{C} 36-\mathrm{C} 35 & -0.9(6) \\ \mathrm{N} 4-\mathrm{C} 21-\mathrm{C} 26-\mathrm{C} 25 & 179(3) & \mathrm{C} 37-\mathrm{C} 31-\mathrm{C} 36-\mathrm{C} 35 & -179.9(4) \\ \mathrm{C} 24-\mathrm{C} 25-\mathrm{C} 26-\mathrm{C} 21 & 1(4) & \mathrm{C} 34-\mathrm{C} 35-\mathrm{C} 36-\mathrm{C} 31 & 0.0(7) \\ \mathrm{C} 23-\mathrm{C} 24-\mathrm{O} 24-\mathrm{C} 27 & 170.6(12) & \mathrm{C} 32-\mathrm{C} 31-\mathrm{C} 37-\mathrm{O} 32 & -4.8(6) \\ \mathrm{C} 25-\mathrm{C} 24-\mathrm{O} 24-\mathrm{C} 27 & -9(3) & \mathrm{C} 36-\mathrm{C} 31-\mathrm{C} 37-\mathrm{O} 32 & 174.2(4) \\ \mathrm{C} 5-\mathrm{N} 4-\mathrm{C} 51-\mathrm{C} 56 & 21(16) & \mathrm{C} 32-\mathrm{C} 31-\mathrm{C} 37-\mathrm{O} 31 & 174.5(4) \\ \mathrm{C} 3-\mathrm{N} 4-\mathrm{C} 51-\mathrm{C} 56 & 151(11) & \mathrm{C} 36-\mathrm{C} 31-\mathrm{C} 37-\mathrm{O} 31 & -6.5(6)\end{array}$

Hydrogen-bond geometry $\left(A,{ }^{\circ}\right)$

\begin{tabular}{lllll}
\hline$D-\mathrm{H} \cdots A$ & $D-\mathrm{H}$ & $\mathrm{H} \cdots A$ & $D \cdots A$ & $D-\mathrm{H} \cdots A$ \\
\hline $\mathrm{N} 1-\mathrm{H} 11 \cdots \mathrm{O} 31$ & $0.78(4)$ & $2.03(4)$ & $2.805(5)$ & $174(5)$ \\
$\mathrm{N} 1-\mathrm{H} 12 \cdots \mathrm{O} 41$ & $0.95(5)$ & $1.86(5)$ & $2.802(5)$ & $172(4)$ \\
$\mathrm{O} 41-\mathrm{H} 41 \cdots \mathrm{O} 32^{\mathrm{i}}$ & $0.79(6)$ & $1.84(6)$ & $2.623(6)$ & $170(6)$ \\
$\mathrm{O} 41-\mathrm{H} 42 \cdots \mathrm{O} 31^{\mathrm{ii}}$ & $0.79(7)$ & $2.00(7)$ & $2.772(5)$ & $169(6)$ \\
$\mathrm{C} 2-\mathrm{H} 2 B \cdots \mathrm{O} 31^{\mathrm{iii}}$ & 0.97 & 2.52 & $3.471(5)$ & 166 \\
$\mathrm{C} 22-\mathrm{H} 22 \cdots \mathrm{Cg} 1^{\mathrm{ii}}$ & 0.93 & 2.52 & $3.471(5)$ & 166 \\
$\mathrm{C} 26-\mathrm{H} 26 \cdots \mathrm{Cg} 1^{\text {iv }}$ & 0.93 & 2.84 & $3.58(2)$ & 137
\end{tabular}

Symmetry codes: (i) $-x+1,-y+1,-z+1$; (ii) $-x+2,-y+1,-z+1$; (iii) $-x+2,-y+2,-z+1$; (iv) $-x+1,-y+2,-z+1$.

4-(4-Methoxyphenyl)piperazin-1-ium 2-hydroxybenzoate (V)

Crystal data

$\mathrm{C}_{11} \mathrm{H}_{17} \mathrm{~N}_{2} \mathrm{O}^{+} \cdot \mathrm{C}_{7} \mathrm{H}_{5} \mathrm{O}_{3}^{-}$

$M_{r}=330.38$

Orthorhombic, $P 22_{1} 22_{1}$

$a=6.5009(8) \AA$

$b=7.9735(9) \AA$

$c=32.155(4) \AA$

$V=1666.8(3) \AA^{3}$

$Z=4$

$F(000)=704$

Data collection

Oxford Diffraction Xcalibur with Sapphire CCD diffractometer

Radiation source: Enhance (Mo) X-ray Source

Graphite monochromator

$\omega$ scans

Absorption correction: multi-scan

(CrysAlis RED; Oxford Diffraction, 2009)

$T_{\min }=0.899, T_{\max }=0.969$
$D_{\mathrm{x}}=1.317 \mathrm{Mg} \mathrm{m}^{-3}$

Mo $K \alpha$ radiation, $\lambda=0.71073 \AA$

Cell parameters from 3564 reflections

$\theta=2.5-27.8^{\circ}$

$\mu=0.09 \mathrm{~mm}^{-1}$

$T=296 \mathrm{~K}$

Block, colourless

$0.42 \times 0.42 \times 0.34 \mathrm{~mm}$

6249 measured reflections

3564 independent reflections

2875 reflections with $I>2 \sigma(I)$

$R_{\text {int }}=0.014$

$\theta_{\text {max }}=27.8^{\circ}, \theta_{\min }=2.5^{\circ}$

$h=-6 \rightarrow 8$

$k=-10 \rightarrow 6$

$l=-38 \rightarrow 41$ 


\section{Refinement}

Refinement on $F^{2}$

Least-squares matrix: full

$R\left[F^{2}>2 \sigma\left(F^{2}\right)\right]=0.041$

$w R\left(F^{2}\right)=0.089$

$S=1.05$

3564 reflections

228 parameters

0 restraints

Primary atom site location: difference Fourier map

Hydrogen site location: mixed
$\mathrm{H}$ atoms treated by a mixture of independent and constrained refinement

$w=1 /\left[\sigma^{2}\left(F_{\mathrm{o}}^{2}\right)+(0.0299 P)^{2}+0.3737 P\right]$ where $P=\left(F_{\mathrm{o}}^{2}+2 F_{\mathrm{c}}^{2}\right) / 3$

$(\Delta / \sigma)_{\max }<0.001$

$\Delta \rho_{\max }=0.14 \mathrm{e} \AA^{-3}$

$\Delta \rho_{\min }=-0.13$ e $\AA^{-3}$

Extinction correction: SHELXL, $\mathrm{Fc}^{*}=\mathrm{kFc}\left[1+0.001 \mathrm{xFc}^{2} \lambda^{3} / \sin (2 \theta)\right]^{-1 / 4}$

Extinction coefficient: 0.0244 (17)

Absolute structure: Flack $x$ determined using 1011 quotients $\left[\left(I^{+}\right)-(I)\right] /\left[\left(I^{+}\right)+(I)\right]$ (Parsons et al., 2013)

\section{Special details}

Experimental. Compound (V). IR $\left(\mathrm{KBr}, \mathrm{cm}^{-1}\right) 3650(\mathrm{OH}), 3040\left(\mathrm{NH}_{2}\right), 2835\left(\mathrm{OCH}_{3}\right), 1571(\mathrm{COO})$. NMR $\left(\mathrm{CDCl}_{3}\right) \delta\left({ }^{1} \mathrm{H}\right)$ $3.31\left(\mathrm{~m}, 8 \mathrm{H}\right.$, piperazine), $3.77\left(\mathrm{~s}, 3 \mathrm{H}, \mathrm{OCH}_{3}\right), 6.85(\mathrm{~m}, 5 \mathrm{H}$, hydroxyphenyl and methoxyphenyl), $6.92(\mathrm{~m}, 1 \mathrm{H}$, hydroxyphenyl), 7.35 (t, 1H, hydroxyphenyl), 7.87 (m, 1H, hydroxyphenyl).

Geometry. All esds (except the esd in the dihedral angle between two 1.s. planes) are estimated using the full covariance matrix. The cell esds are taken into account individually in the estimation of esds in distances, angles and torsion angles; correlations between esds in cell parameters are only used when they are defined by crystal symmetry. An approximate (isotropic) treatment of cell esds is used for estimating esds involving l.s. planes.

Fractional atomic coordinates and isotropic or equivalent isotropic displacement parameters $\left(\AA^{2}\right)$

\begin{tabular}{lllll}
\hline & $x$ & $y$ & $z$ & $U_{\text {iso }} * / U_{\text {eq }}$ \\
\hline $\mathrm{N} 1$ & $0.5261(3)$ & $0.3567(3)$ & $0.21309(7)$ & $0.0468(5)$ \\
$\mathrm{H} 11$ & $0.616(4)$ & $0.369(4)$ & $0.2367(9)$ & $0.056^{*}$ \\
$\mathrm{H} 12$ & $0.452(4)$ & $0.254(4)$ & $0.2144(8)$ & $0.056^{*}$ \\
$\mathrm{C} 2$ & $0.6568(4)$ & $0.3607(4)$ & $0.17563(8)$ & $0.0488(6)$ \\
$\mathrm{H} 2 \mathrm{~A}$ & 0.7532 & 0.2680 & 0.1765 & $0.059^{*}$ \\
$\mathrm{H} 2 \mathrm{~B}$ & 0.7353 & 0.4642 & 0.1753 & $0.059^{*}$ \\
$\mathrm{C} 3$ & $0.5299(4)$ & $0.3490(3)$ & $0.13672(8)$ & $0.0443(6)$ \\
$\mathrm{H} 3 \mathrm{~A}$ & 0.6189 & 0.3643 & 0.1128 & $0.053^{*}$ \\
$\mathrm{H} 3 \mathrm{~B}$ & 0.4705 & 0.2377 & 0.1349 & $0.053^{*}$ \\
$\mathrm{~N} 4$ & $0.3654(3)$ & $0.4727(3)$ & $0.13512(6)$ & $0.0381(5)$ \\
$\mathrm{C} 5$ & $0.2422(4)$ & $0.4788(3)$ & $0.17339(7)$ & $0.0426(6)$ \\
$\mathrm{H} 5 \mathrm{~A}$ & 0.1606 & 0.3773 & 0.1755 & $0.051^{*}$ \\
$\mathrm{H} 5 \mathrm{~B}$ & 0.1485 & 0.5733 & 0.1720 & $0.051^{*}$ \\
$\mathrm{C} 6$ & $0.3752(4)$ & $0.4953(3)$ & $0.21130(8)$ & $0.0460(6)$ \\
$\mathrm{H} 6 \mathrm{~A}$ & 0.4472 & 0.6018 & 0.2106 & $0.055^{*}$ \\
$\mathrm{H} 6 \mathrm{~B}$ & 0.2898 & 0.4933 & 0.2360 & $0.055^{*}$ \\
$\mathrm{C} 21$ & $0.2490(4)$ & $0.4737(3)$ & $0.09771(7)$ & $0.0373(5)$ \\
$\mathrm{C} 22$ & $0.3077(4)$ & $0.3820(4)$ & $0.06280(8)$ & $0.0515(7)$ \\
$\mathrm{H} 22$ & 0.4232 & 0.3134 & 0.0642 & $0.062^{*}$ \\
$\mathrm{C} 23$ & $0.1976(5)$ & $0.3911(4)$ & $0.02617(8)$ & $0.0544(7)$ \\
H23 & 0.2412 & 0.3295 & 0.0033 & $0.065^{*}$ \\
C24 & $0.0260(4)$ & $0.4886(3)$ & $0.02292(8)$ & $0.0460(6)$ \\
C25 & $-0.0385(4)$ & $0.5770(4)$ & $0.05738(8)$ & $0.0529(7)$ \\
& & & &
\end{tabular}




$\begin{array}{lllll}\mathrm{H} 25 & -0.1573 & 0.6418 & 0.0560 & 0.063^{*} \\ \mathrm{C} 26 & 0.0728(4) & 0.5697(4) & 0.09394(8) & 0.0503(7) \\ \mathrm{H} 26 & 0.0277 & 0.6312 & 0.1168 & 0.060^{*} \\ \mathrm{O} 24 & -0.0704(3) & 0.4899(3) & -0.01523(5) & 0.0638(6) \\ \mathrm{C} 27 & -0.2374(6) & 0.6009(5) & -0.02052(10) & 0.0833(11) \\ \mathrm{H} 27 \mathrm{~A} & -0.2851 & 0.5953 & -0.0487 & 0.125^{*} \\ \mathrm{H} 27 \mathrm{~B} & -0.1938 & 0.7133 & -0.0144 & 0.125^{*} \\ \mathrm{H} 27 \mathrm{C} & -0.3469 & 0.5697 & -0.0020 & 0.125^{*} \\ \mathrm{C} 31 & 0.9904(4) & 0.4542(3) & 0.32938(7) & 0.0370(5) \\ \mathrm{C} 32 & 1.1705(4) & 0.3584(3) & 0.32627(7) & 0.0394(5) \\ \mathrm{C} 33 & 1.3127(4) & 0.3600(4) & 0.35834(8) & 0.0501(7) \\ \mathrm{H} 33 & 1.4324 & 0.2967 & 0.3561 & 0.060^{*} \\ \mathrm{C} 34 & 1.2782(5) & 0.4544(4) & 0.39334(8) & 0.0557(7) \\ \mathrm{H} 34 & 1.3746 & 0.4546 & 0.4147 & 0.067^{*} \\ \mathrm{C} 35 & 1.1023(5) & 0.5484(4) & 0.39698(8) & 0.0584(8) \\ \mathrm{H} 35 & 1.0790 & 0.6119 & 0.4208 & 0.070^{*} \\ \mathrm{C} 36 & 0.9613(4) & 0.5480(3) & 0.36539(8) & 0.0501(7) \\ \mathrm{H} 36 & 0.8427 & 0.6123 & 0.3681 & 0.060^{*} \\ \text { O33 } & 1.2074(3) & 0.2609(2) & 0.29254(6) & 0.0548(5) \\ \mathrm{H} 33 \mathrm{~A} & 1.082(5) & 0.275(4) & 0.2766(10) & 0.082^{*} \\ \mathrm{C} 37 & 0.8348(4) & 0.4561(3) & 0.29516(8) & 0.0453(6) \\ \text { O31 } & 0.8614(3) & 0.3519(3) & 0.26569(6) & 0.0601(6) \\ \text { O32 } & 0.6867(3) & 0.5531(3) & 0.29674(7) & 0.0682(6) \\ & & & & \end{array}$

Atomic displacement parameters $\left(\AA^{2}\right)$

\begin{tabular}{lllllll}
\hline & $U^{11}$ & $U^{22}$ & $U^{33}$ & $U^{12}$ & $U^{13}$ & $U^{23}$ \\
\hline $\mathrm{N} 1$ & $0.0464(13)$ & $0.0489(12)$ & $0.0452(12)$ & $-0.0102(11)$ & $-0.0143(11)$ & $0.0037(11)$ \\
$\mathrm{C} 2$ & $0.0363(13)$ & $0.0520(15)$ & $0.0581(15)$ & $-0.0003(12)$ & $-0.0058(13)$ & $-0.0004(14)$ \\
$\mathrm{C} 3$ & $0.0364(13)$ & $0.0475(14)$ & $0.0489(14)$ & $0.0030(12)$ & $0.0007(11)$ & $-0.0024(12)$ \\
$\mathrm{N} 4$ & $0.0353(10)$ & $0.0416(11)$ & $0.0374(10)$ & $0.0037(9)$ & $-0.0007(8)$ & $-0.0024(9)$ \\
$\mathrm{C} 5$ & $0.0388(12)$ & $0.0494(14)$ & $0.0395(12)$ & $0.0027(12)$ & $0.0008(11)$ & $-0.0041(11)$ \\
$\mathrm{C} 6$ & $0.0484(14)$ & $0.0492(15)$ & $0.0404(13)$ & $-0.0030(13)$ & $0.0000(12)$ & $-0.0022(12)$ \\
$\mathrm{C} 21$ & $0.0396(12)$ & $0.0350(12)$ & $0.0374(12)$ & $-0.0018(11)$ & $0.0015(10)$ & $-0.0011(10)$ \\
$\mathrm{C} 22$ & $0.0559(16)$ & $0.0543(16)$ & $0.0444(14)$ & $0.0172(14)$ & $0.0005(13)$ & $-0.0062(13)$ \\
$\mathrm{C} 23$ & $0.0699(19)$ & $0.0551(17)$ & $0.0381(13)$ & $0.0109(15)$ & $0.0013(14)$ & $-0.0102(12)$ \\
$\mathrm{C} 24$ & $0.0555(15)$ & $0.0431(14)$ & $0.0393(13)$ & $-0.0032(13)$ & $-0.0070(12)$ & $0.0005(11)$ \\
$\mathrm{C} 25$ & $0.0501(15)$ & $0.0582(17)$ & $0.0503(16)$ & $0.0139(14)$ & $-0.0088(13)$ & $-0.0047(13)$ \\
$\mathrm{C} 26$ & $0.0523(15)$ & $0.0567(17)$ & $0.0418(14)$ & $0.0153(14)$ & $-0.0030(12)$ & $-0.0121(12)$ \\
$\mathrm{O} 24$ & $0.0793(14)$ & $0.0684(14)$ & $0.0435(10)$ & $0.0051(12)$ & $-0.0190(10)$ & $-0.0036(9)$ \\
$\mathrm{C} 27$ & $0.080(2)$ & $0.102(3)$ & $0.068(2)$ & $0.014(2)$ & $-0.0331(19)$ & $0.000(2)$ \\
$\mathrm{C} 31$ & $0.0382(12)$ & $0.0331(12)$ & $0.0396(13)$ & $-0.0030(10)$ & $-0.0004(10)$ & $0.0076(10)$ \\
$\mathrm{C} 32$ & $0.0416(12)$ & $0.0353(12)$ & $0.0413(13)$ & $-0.0017(11)$ & $-0.0006(12)$ & $0.0047(11)$ \\
$\mathrm{C} 33$ & $0.0398(14)$ & $0.0552(16)$ & $0.0554(16)$ & $0.0006(13)$ & $-0.0092(13)$ & $0.0093(14)$ \\
$\mathrm{C} 34$ & $0.0586(17)$ & $0.0642(18)$ & $0.0442(15)$ & $-0.0139(16)$ & $-0.0139(14)$ & $0.0084(14)$ \\
$\mathrm{C} 35$ & $0.076(2)$ & $0.0595(18)$ & $0.0401(15)$ & $-0.0090(17)$ & $0.0015(14)$ & $-0.0046(13)$ \\
$\mathrm{C} 36$ & $0.0524(15)$ & $0.0472(15)$ & $0.0506(15)$ & $0.0064(14)$ & $0.0064(13)$ & $0.0028(12)$ \\
O33 & $0.0547(12)$ & $0.0555(12)$ & $0.0542(11)$ & $0.0139(10)$ & $-0.0054(10)$ & $-0.0087(9)$
\end{tabular}




\begin{tabular}{lllllll}
$\mathrm{C} 37$ & $0.0431(14)$ & $0.0409(14)$ & $0.0517(15)$ & $-0.0037(13)$ & $-0.0069(12)$ & $0.0127(12)$ \\
$\mathrm{O} 31$ & $0.0639(13)$ & $0.0586(12)$ & $0.0580(12)$ & $0.0036(11)$ & $-0.0245(10)$ & $-0.0057(10)$ \\
$\mathrm{O} 32$ & $0.0524(11)$ & $0.0730(14)$ & $0.0793(14)$ & $0.0200(11)$ & $-0.0115(11)$ & $0.0136(11)$ \\
\hline
\end{tabular}

Geometric parameters $\left(A,{ }^{o}\right)$

\begin{tabular}{|c|c|c|c|}
\hline $\mathrm{N} 1-\mathrm{C} 2$ & $1.474(3)$ & $\mathrm{C} 24-\mathrm{C} 25$ & $1.379(4)$ \\
\hline $\mathrm{N} 1-\mathrm{C} 6$ & $1.479(3)$ & $\mathrm{C} 25-\mathrm{C} 26$ & $1.382(4)$ \\
\hline $\mathrm{N} 1-\mathrm{H} 11$ & $0.96(3)$ & $\mathrm{C} 25-\mathrm{H} 25$ & 0.9300 \\
\hline $\mathrm{N} 1-\mathrm{H} 12$ & $0.95(3)$ & $\mathrm{C} 26-\mathrm{H} 26$ & 0.9300 \\
\hline $\mathrm{C} 2-\mathrm{C} 3$ & $1.502(3)$ & $\mathrm{O} 24-\mathrm{C} 27$ & $1.411(4)$ \\
\hline $\mathrm{C} 2-\mathrm{H} 2 \mathrm{~A}$ & 0.9700 & $\mathrm{C} 27-\mathrm{H} 27 \mathrm{~A}$ & 0.9600 \\
\hline $\mathrm{C} 2-\mathrm{H} 2 \mathrm{~B}$ & 0.9700 & $\mathrm{C} 27-\mathrm{H} 27 \mathrm{~B}$ & 0.9600 \\
\hline $\mathrm{C} 3-\mathrm{N} 4$ & $1.456(3)$ & $\mathrm{C} 27-\mathrm{H} 27 \mathrm{C}$ & 0.9600 \\
\hline $\mathrm{C} 3-\mathrm{H} 3 \mathrm{~A}$ & 0.9700 & $\mathrm{C} 31-\mathrm{C} 36$ & $1.391(3)$ \\
\hline $\mathrm{C} 3-\mathrm{H} 3 \mathrm{~B}$ & 0.9700 & $\mathrm{C} 31-\mathrm{C} 32$ & $1.401(3)$ \\
\hline $\mathrm{N} 4-\mathrm{C} 21$ & $1.421(3)$ & $\mathrm{C} 31-\mathrm{C} 37$ & $1.495(3)$ \\
\hline $\mathrm{N} 4-\mathrm{C} 5$ & $1.469(3)$ & $\mathrm{C} 32-\mathrm{O} 33$ & $1.356(3)$ \\
\hline $\mathrm{C} 5-\mathrm{C} 6$ & $1.500(3)$ & $\mathrm{C} 32-\mathrm{C} 33$ & $1.385(3)$ \\
\hline $\mathrm{C} 5-\mathrm{H} 5 \mathrm{~A}$ & 0.9700 & $\mathrm{C} 33-\mathrm{C} 34$ & $1.372(4)$ \\
\hline C5-H5B & 0.9700 & $\mathrm{C} 33-\mathrm{H} 33$ & 0.9300 \\
\hline C6-H6A & 0.9700 & $\mathrm{C} 34-\mathrm{C} 35$ & $1.372(4)$ \\
\hline C6-H6B & 0.9700 & C $34-\mathrm{H} 34$ & 0.9300 \\
\hline $\mathrm{C} 21-\mathrm{C} 26$ & $1.383(3)$ & $\mathrm{C} 35-\mathrm{C} 36$ & $1.368(4)$ \\
\hline $\mathrm{C} 21-\mathrm{C} 22$ & $1.393(3)$ & $\mathrm{C} 35-\mathrm{H} 35$ & 0.9300 \\
\hline $\mathrm{C} 22-\mathrm{C} 23$ & $1.380(4)$ & $\mathrm{C} 36-\mathrm{H} 36$ & 0.9300 \\
\hline $\mathrm{C} 22-\mathrm{H} 22$ & 0.9300 & $\mathrm{O} 33-\mathrm{H} 33 \mathrm{~A}$ & $0.97(3)$ \\
\hline $\mathrm{C} 23-\mathrm{C} 24$ & $1.364(4)$ & $\mathrm{C} 37-\mathrm{O} 32$ & $1.236(3)$ \\
\hline $\mathrm{C} 23-\mathrm{H} 23$ & 0.9300 & $\mathrm{C} 37-\mathrm{O} 31$ & $1.272(3)$ \\
\hline $\mathrm{C} 24-\mathrm{O} 24$ & $1.378(3)$ & & \\
\hline $\mathrm{C} 2-\mathrm{N} 1-\mathrm{C} 6$ & $109.5(2)$ & $\mathrm{C} 24-\mathrm{C} 23-\mathrm{H} 23$ & 119.3 \\
\hline $\mathrm{C} 2-\mathrm{N} 1-\mathrm{H} 11$ & $107.1(16)$ & $\mathrm{C} 22-\mathrm{C} 23-\mathrm{H} 23$ & 119.3 \\
\hline $\mathrm{C} 6-\mathrm{N} 1-\mathrm{H} 11$ & $110.9(17)$ & $\mathrm{C} 23-\mathrm{C} 24-\mathrm{O} 24$ & $116.4(2)$ \\
\hline $\mathrm{C} 2-\mathrm{N} 1-\mathrm{H} 12$ & $110.3(16)$ & $\mathrm{C} 23-\mathrm{C} 24-\mathrm{C} 25$ & $118.6(2)$ \\
\hline $\mathrm{C} 6-\mathrm{N} 1-\mathrm{H} 12$ & $108.2(16)$ & $\mathrm{O} 24-\mathrm{C} 24-\mathrm{C} 25$ & $125.0(2)$ \\
\hline $\mathrm{H} 11-\mathrm{N} 1-\mathrm{H} 12$ & $111(2)$ & $\mathrm{C} 24-\mathrm{C} 25-\mathrm{C} 26$ & $120.2(2)$ \\
\hline $\mathrm{N} 1-\mathrm{C} 2-\mathrm{C} 3$ & $111.3(2)$ & $\mathrm{C} 24-\mathrm{C} 25-\mathrm{H} 25$ & 119.9 \\
\hline $\mathrm{N} 1-\mathrm{C} 2-\mathrm{H} 2 \mathrm{~A}$ & 109.4 & $\mathrm{C} 26-\mathrm{C} 25-\mathrm{H} 25$ & 119.9 \\
\hline $\mathrm{C} 3-\mathrm{C} 2-\mathrm{H} 2 \mathrm{~A}$ & 109.4 & $\mathrm{C} 25-\mathrm{C} 26-\mathrm{C} 21$ & $122.1(2)$ \\
\hline $\mathrm{N} 1-\mathrm{C} 2-\mathrm{H} 2 \mathrm{~B}$ & 109.4 & $\mathrm{C} 25-\mathrm{C} 26-\mathrm{H} 26$ & 118.9 \\
\hline $\mathrm{C} 3-\mathrm{C} 2-\mathrm{H} 2 \mathrm{~B}$ & 109.4 & $\mathrm{C} 21-\mathrm{C} 26-\mathrm{H} 26$ & 118.9 \\
\hline $\mathrm{H} 2 \mathrm{~A}-\mathrm{C} 2-\mathrm{H} 2 \mathrm{~B}$ & 108.0 & $\mathrm{C} 24-\mathrm{O} 24-\mathrm{C} 27$ & $117.5(2)$ \\
\hline $\mathrm{N} 4-\mathrm{C} 3-\mathrm{C} 2$ & $113.0(2)$ & $\mathrm{O} 24-\mathrm{C} 27-\mathrm{H} 27 \mathrm{~A}$ & 109.5 \\
\hline $\mathrm{N} 4-\mathrm{C} 3-\mathrm{H} 3 \mathrm{~A}$ & 109.0 & $\mathrm{O} 24-\mathrm{C} 27-\mathrm{H} 27 \mathrm{~B}$ & 109.5 \\
\hline $\mathrm{C} 2-\mathrm{C} 3-\mathrm{H} 3 \mathrm{~A}$ & 109.0 & $\mathrm{H} 27 \mathrm{~A}-\mathrm{C} 27-\mathrm{H} 27 \mathrm{~B}$ & 109.5 \\
\hline $\mathrm{N} 4-\mathrm{C} 3-\mathrm{H} 3 \mathrm{~B}$ & 109.0 & $\mathrm{O} 24-\mathrm{C} 27-\mathrm{H} 27 \mathrm{C}$ & 109.5 \\
\hline $\mathrm{C} 2-\mathrm{C} 3-\mathrm{H} 3 \mathrm{~B}$ & 109.0 & $\mathrm{H} 27 \mathrm{~A}-\mathrm{C} 27-\mathrm{H} 27 \mathrm{C}$ & 109.5 \\
\hline
\end{tabular}




\begin{tabular}{|c|c|c|c|}
\hline $\mathrm{H} 3 \mathrm{~A}-\mathrm{C} 3-\mathrm{H} 3 \mathrm{~B}$ & 107.8 & $\mathrm{H} 27 \mathrm{~B}-\mathrm{C} 27-\mathrm{H} 27 \mathrm{C}$ & 109.5 \\
\hline $\mathrm{C} 21-\mathrm{N} 4-\mathrm{C} 3$ & $115.14(18)$ & $\mathrm{C} 36-\mathrm{C} 31-\mathrm{C} 32$ & $117.8(2)$ \\
\hline $\mathrm{C} 21-\mathrm{N} 4-\mathrm{C} 5$ & $114.77(18)$ & $\mathrm{C} 36-\mathrm{C} 31-\mathrm{C} 37$ & $121.0(2)$ \\
\hline $\mathrm{C} 3-\mathrm{N} 4-\mathrm{C} 5$ & $113.17(19)$ & $\mathrm{C} 32-\mathrm{C} 31-\mathrm{C} 37$ & $121.2(2)$ \\
\hline $\mathrm{N} 4-\mathrm{C} 5-\mathrm{C} 6$ & $111.69(19)$ & $\mathrm{O} 33-\mathrm{C} 32-\mathrm{C} 33$ & $118.8(2)$ \\
\hline $\mathrm{N} 4-\mathrm{C} 5-\mathrm{H} 5 \mathrm{~A}$ & 109.3 & $\mathrm{O} 33-\mathrm{C} 32-\mathrm{C} 31$ & $121.2(2)$ \\
\hline $\mathrm{C} 6-\mathrm{C} 5-\mathrm{H} 5 \mathrm{~A}$ & 109.3 & $\mathrm{C} 33-\mathrm{C} 32-\mathrm{C} 31$ & $120.0(2)$ \\
\hline $\mathrm{N} 4-\mathrm{C} 5-\mathrm{H} 5 \mathrm{~B}$ & 109.3 & $\mathrm{C} 34-\mathrm{C} 33-\mathrm{C} 32$ & $120.4(3)$ \\
\hline $\mathrm{C} 6-\mathrm{C} 5-\mathrm{H} 5 \mathrm{~B}$ & 109.3 & $\mathrm{C} 34-\mathrm{C} 33-\mathrm{H} 33$ & 119.8 \\
\hline $\mathrm{H} 5 \mathrm{~A}-\mathrm{C} 5-\mathrm{H} 5 \mathrm{~B}$ & 107.9 & $\mathrm{C} 32-\mathrm{C} 33-\mathrm{H} 33$ & 119.8 \\
\hline $\mathrm{N} 1-\mathrm{C} 6-\mathrm{C} 5$ & $110.4(2)$ & $\mathrm{C} 35-\mathrm{C} 34-\mathrm{C} 33$ & $120.4(3)$ \\
\hline $\mathrm{N} 1-\mathrm{C} 6-\mathrm{H} 6 \mathrm{~A}$ & 109.6 & $\mathrm{C} 35-\mathrm{C} 34-\mathrm{H} 34$ & 119.8 \\
\hline $\mathrm{C} 5-\mathrm{C} 6-\mathrm{H} 6 \mathrm{~A}$ & 109.6 & $\mathrm{C} 33-\mathrm{C} 34-\mathrm{H} 34$ & 119.8 \\
\hline $\mathrm{N} 1-\mathrm{C} 6-\mathrm{H} 6 \mathrm{~B}$ & 109.6 & $\mathrm{C} 36-\mathrm{C} 35-\mathrm{C} 34$ & $119.6(3)$ \\
\hline $\mathrm{C} 5-\mathrm{C} 6-\mathrm{H} 6 \mathrm{~B}$ & 109.6 & $\mathrm{C} 36-\mathrm{C} 35-\mathrm{H} 35$ & 120.2 \\
\hline $\mathrm{H} 6 \mathrm{~A}-\mathrm{C} 6-\mathrm{H} 6 \mathrm{~B}$ & 108.1 & $\mathrm{C} 34-\mathrm{C} 35-\mathrm{H} 35$ & 120.2 \\
\hline $\mathrm{C} 26-\mathrm{C} 21-\mathrm{C} 22$ & $116.5(2)$ & $\mathrm{C} 35-\mathrm{C} 36-\mathrm{C} 31$ & $121.9(3)$ \\
\hline $\mathrm{C} 26-\mathrm{C} 21-\mathrm{N} 4$ & $121.2(2)$ & $\mathrm{C} 35-\mathrm{C} 36-\mathrm{H} 36$ & 119.1 \\
\hline $\mathrm{C} 22-\mathrm{C} 21-\mathrm{N} 4$ & $122.2(2)$ & $\mathrm{C} 31-\mathrm{C} 36-\mathrm{H} 36$ & 119.1 \\
\hline $\mathrm{C} 23-\mathrm{C} 22-\mathrm{C} 21$ & $121.2(3)$ & $\mathrm{C} 32-\mathrm{O} 33-\mathrm{H} 33 \mathrm{~A}$ & $102(2)$ \\
\hline $\mathrm{C} 23-\mathrm{C} 22-\mathrm{H} 22$ & 119.4 & $\mathrm{O} 32-\mathrm{C} 37-\mathrm{O} 31$ & $123.0(2)$ \\
\hline $\mathrm{C} 21-\mathrm{C} 22-\mathrm{H} 22$ & 119.4 & $\mathrm{O} 32-\mathrm{C} 37-\mathrm{C} 31$ & $120.2(3)$ \\
\hline $\mathrm{C} 24-\mathrm{C} 23-\mathrm{C} 22$ & $121.3(2)$ & $\mathrm{O} 31-\mathrm{C} 37-\mathrm{C} 31$ & $116.7(2)$ \\
\hline $\mathrm{C} 6-\mathrm{N} 1-\mathrm{C} 2-\mathrm{C} 3$ & $-57.5(3)$ & $\mathrm{C} 22-\mathrm{C} 21-\mathrm{C} 26-\mathrm{C} 25$ & $-1.0(4)$ \\
\hline $\mathrm{N} 1-\mathrm{C} 2-\mathrm{C} 3-\mathrm{N} 4$ & $52.5(3)$ & $\mathrm{N} 4-\mathrm{C} 21-\mathrm{C} 26-\mathrm{C} 25$ & $177.7(3)$ \\
\hline $\mathrm{C} 2-\mathrm{C} 3-\mathrm{N} 4-\mathrm{C} 21$ & $176.2(2)$ & $\mathrm{C} 23-\mathrm{C} 24-\mathrm{O} 24-\mathrm{C} 27$ & $-174.6(3)$ \\
\hline $\mathrm{C} 2-\mathrm{C} 3-\mathrm{N} 4-\mathrm{C} 5$ & $-49.1(3)$ & $\mathrm{C} 25-\mathrm{C} 24-\mathrm{O} 24-\mathrm{C} 27$ & $5.7(4)$ \\
\hline $\mathrm{C} 21-\mathrm{N} 4-\mathrm{C} 5-\mathrm{C} 6$ & $-174.1(2)$ & $\mathrm{C} 36-\mathrm{C} 31-\mathrm{C} 32-\mathrm{O} 33$ & $178.5(2)$ \\
\hline $\mathrm{C} 3-\mathrm{N} 4-\mathrm{C} 5-\mathrm{C} 6$ & $51.0(3)$ & $\mathrm{C} 37-\mathrm{C} 31-\mathrm{C} 32-\mathrm{O} 33$ & $-1.6(3)$ \\
\hline $\mathrm{C} 2-\mathrm{N} 1-\mathrm{C} 6-\mathrm{C} 5$ & $59.7(3)$ & $\mathrm{C} 36-\mathrm{C} 31-\mathrm{C} 32-\mathrm{C} 33$ & $-0.3(3)$ \\
\hline $\mathrm{N} 4-\mathrm{C} 5-\mathrm{C} 6-\mathrm{N} 1$ & $-56.4(3)$ & $\mathrm{C} 37-\mathrm{C} 31-\mathrm{C} 32-\mathrm{C} 33$ & $179.5(2)$ \\
\hline $\mathrm{C} 3-\mathrm{N} 4-\mathrm{C} 21-\mathrm{C} 26$ & $171.8(2)$ & $\mathrm{O} 33-\mathrm{C} 32-\mathrm{C} 33-\mathrm{C} 34$ & $-178.6(2)$ \\
\hline $\mathrm{C} 5-\mathrm{N} 4-\mathrm{C} 21-\mathrm{C} 26$ & $37.8(3)$ & $\mathrm{C} 31-\mathrm{C} 32-\mathrm{C} 33-\mathrm{C} 34$ & $0.3(4)$ \\
\hline $\mathrm{C} 3-\mathrm{N} 4-\mathrm{C} 21-\mathrm{C} 22$ & $-9.6(3)$ & $\mathrm{C} 32-\mathrm{C} 33-\mathrm{C} 34-\mathrm{C} 35$ & $0.0(4)$ \\
\hline $\mathrm{C} 5-\mathrm{N} 4-\mathrm{C} 21-\mathrm{C} 22$ & $-143.6(2)$ & $\mathrm{C} 33-\mathrm{C} 34-\mathrm{C} 35-\mathrm{C} 36$ & $-0.2(4)$ \\
\hline $\mathrm{C} 26-\mathrm{C} 21-\mathrm{C} 22-\mathrm{C} 23$ & $1.7(4)$ & $\mathrm{C} 34-\mathrm{C} 35-\mathrm{C} 36-\mathrm{C} 31$ & $0.2(4)$ \\
\hline $\mathrm{N} 4-\mathrm{C} 21-\mathrm{C} 22-\mathrm{C} 23$ & $-176.9(3)$ & $\mathrm{C} 32-\mathrm{C} 31-\mathrm{C} 36-\mathrm{C} 35$ & $0.1(4)$ \\
\hline $\mathrm{C} 21-\mathrm{C} 22-\mathrm{C} 23-\mathrm{C} 24$ & $-0.8(4)$ & $\mathrm{C} 37-\mathrm{C} 31-\mathrm{C} 36-\mathrm{C} 35$ & $-179.8(2)$ \\
\hline $\mathrm{C} 22-\mathrm{C} 23-\mathrm{C} 24-\mathrm{O} 24$ & $179.2(3)$ & $\mathrm{C} 36-\mathrm{C} 31-\mathrm{C} 37-\mathrm{O} 32$ & $6.6(4)$ \\
\hline $\mathrm{C} 22-\mathrm{C} 23-\mathrm{C} 24-\mathrm{C} 25$ & $-1.0(4)$ & $\mathrm{C} 32-\mathrm{C} 31-\mathrm{C} 37-\mathrm{O} 32$ & $-173.2(2)$ \\
\hline $\mathrm{C} 23-\mathrm{C} 24-\mathrm{C} 25-\mathrm{C} 26$ & $1.8(4)$ & $\mathrm{C} 36-\mathrm{C} 31-\mathrm{C} 37-\mathrm{O} 31$ & $-171.9(2)$ \\
\hline $\mathrm{O} 24-\mathrm{C} 24-\mathrm{C} 25-\mathrm{C} 26$ & $-178.5(3)$ & $\mathrm{C} 32-\mathrm{C} 31-\mathrm{C} 37-\mathrm{O} 31$ & $8.3(3)$ \\
\hline $\mathrm{C} 24-\mathrm{C} 25-\mathrm{C} 26-\mathrm{C} 21$ & $-0.7(5)$ & & \\
\hline
\end{tabular}


Hydrogen-bond geometry $\left(\AA,{ }^{\circ}\right)$

\begin{tabular}{lllll}
\hline$D-\mathrm{H} \cdots A$ & $D-\mathrm{H}$ & $\mathrm{H} \cdots A$ & $D \cdots A$ & $D-\mathrm{H} \cdots A$ \\
\hline $\mathrm{N} 1-\mathrm{H} 11 \cdots \mathrm{O} 31$ & $0.96(3)$ & $1.85(3)$ & $2.759(3)$ & $156(3)$ \\
$\mathrm{N} 1-\mathrm{H} 11 \cdots \mathrm{O} 32$ & $0.96(3)$ & $2.47(3)$ & $3.283(3)$ & $142(2)$ \\
$\mathrm{N} 1-\mathrm{H} 12 \cdots \mathrm{O} 32^{\mathrm{i}}$ & $0.95(3)$ & $1.87(3)$ & $2.806(3)$ & $166(2)$ \\
$\mathrm{O} 33-\mathrm{H} 33 A \cdots \mathrm{O} 31$ & $0.97(3)$ & $1.60(3)$ & $2.516(3)$ & $156(3)$ \\
$\mathrm{C} 6-\mathrm{H} 6 A \cdots \mathrm{O} 33^{\mathrm{ii}}$ & 0.97 & 2.58 & $3.444(3)$ & 148 \\
$\mathrm{C} 2-\mathrm{H} 2 A \cdots C g 1^{\mathrm{iii}}$ & 0.97 & 2.88 & $3.711(3)$ & 144 \\
$\mathrm{C} 26-\mathrm{H} 26 \cdots C g 1^{\mathrm{iv}}$ & 0.93 & 2.87 & $3.642(3)$ & 141
\end{tabular}

Symmetry codes: (i) $-x+1, y-1 / 2,-z+1 / 2$; (ii) $-x+2, y+1 / 2,-z+1 / 2$; (iii) $-x+2, y-1 / 2,-z+1 / 2$; (iv) $-x+1, y+1 / 2,-z+1 / 2$.

4-(4-Methoxyphenyl)piperazin-1-ium pyridine-3-carboxylate (VI)

\section{Crystal data}

$\mathrm{C}_{11} \mathrm{H}_{17} \mathrm{~N}_{2} \mathrm{O}^{+} \cdot \mathrm{C}_{6} \mathrm{H}_{4} \mathrm{NO}_{2}^{-}$

$M_{r}=315.37$

Orthorhombic, $\mathrm{Pbca}$

$a=9.2817(7) \AA$

$b=11.2905(7) \AA$

$c=30.309(2) \AA$

$V=3176.2(4) \AA^{3}$

$Z=8$

$F(000)=1344$

\section{Data collection}

Oxford Diffraction Xcalibur with Sapphire CCD diffractometer

Radiation source: Enhance (Mo) X-ray Source

Graphite monochromator

$\omega$ scans

Absorption correction: multi-scan

(CrysAlis RED; Oxford Diffraction, 2009)

$T_{\min }=0.879, T_{\max }=0.968$

\section{Refinement}

Refinement on $F^{2}$

Least-squares matrix: full

$R\left[F^{2}>2 \sigma\left(F^{2}\right)\right]=0.048$

$w R\left(F^{2}\right)=0.119$

$S=1.03$

3593 reflections

215 parameters

0 restraints

Primary atom site location: difference Fourier map

Hydrogen site location: mixed
$D_{\mathrm{x}}=1.319 \mathrm{Mg} \mathrm{m}^{-3}$

Mo $K \alpha$ radiation, $\lambda=0.71073 \AA$

Cell parameters from 3593 reflections

$\theta=2.6-27.9^{\circ}$

$\mu=0.09 \mathrm{~mm}^{-1}$

$T=296 \mathrm{~K}$

Block, colourless

$0.46 \times 0.42 \times 0.36 \mathrm{~mm}$

22154 measured reflections

3593 independent reflections

2616 reflections with $I>2 \sigma(I)$

$R_{\text {int }}=0.028$

$\theta_{\text {max }}=27.9^{\circ}, \theta_{\min }=2.6^{\circ}$

$h=-11 \rightarrow 11$

$k=-14 \rightarrow 13$

$l=-38 \rightarrow 35$

$\mathrm{H}$ atoms treated by a mixture of independent and constrained refinement

$w=1 /\left[\sigma^{2}\left(F_{\mathrm{o}}^{2}\right)+(0.0416 P)^{2}+1.5726 P\right]$ where $P=\left(F_{\mathrm{o}}^{2}+2 F_{\mathrm{c}}{ }^{2}\right) / 3$

$(\Delta / \sigma)_{\max }=0.001$

$\Delta \rho_{\max }=0.19 \mathrm{e} \AA^{-3}$

$\Delta \rho_{\min }=-0.16$ e $\AA^{-3}$

Extinction correction: SHELXL, $\mathrm{Fc}^{*}=\mathrm{kFc}\left[1+0.001 \mathrm{xFc}^{2} \lambda^{3} / \sin (2 \theta)\right]^{-1 / 4}$

Extinction coefficient: 0.0074 (6)

\section{Special details}

Experimental. Compound (VI). IR $\left(\mathrm{KBr}, \mathrm{cm}^{-1}\right) 3040\left(\mathrm{NH}_{2}\right), 2829\left(\mathrm{OCH}_{3}\right), 1584(\mathrm{COO})$. NMR $\left(\mathrm{CDCl}_{3}\right) \delta\left({ }^{1} \mathrm{H}\right) 3.27(\mathrm{~m}$, $4 \mathrm{H}$, piperazine), $3.34\left(\mathrm{~m}, 4 \mathrm{H}\right.$, piperazine), $3.77\left(\mathrm{~s}, 3 \mathrm{H}, \mathrm{OCH}_{3}\right), 6.90(\mathrm{~m}, 4 \mathrm{H}$, methoxyphenyl), $7.33(\mathrm{~m}, 1 \mathrm{H}$, nicotinate), $8.67(\mathrm{~m}, 2 \mathrm{H}$, nicotinate), $9.24(\mathrm{~m}, 1 \mathrm{H}$, nicotinate). 
Geometry. All esds (except the esd in the dihedral angle between two 1.s. planes) are estimated using the full covariance matrix. The cell esds are taken into account individually in the estimation of esds in distances, angles and torsion angles; correlations between esds in cell parameters are only used when they are defined by crystal symmetry. An approximate (isotropic) treatment of cell esds is used for estimating esds involving 1.s. planes.

Fractional atomic coordinates and isotropic or equivalent isotropic displacement parameters $\left(\hat{A}^{2}\right)$

\begin{tabular}{|c|c|c|c|c|}
\hline & $x$ & $y$ & $z$ & $U_{\text {iso }} * / U_{\text {eq }}$ \\
\hline N1 & $0.66467(17)$ & $0.63416(13)$ & $0.53807(5)$ & 0.0405 (4) \\
\hline H12 & $0.711(2)$ & $0.5701(18)$ & $0.5241(6)$ & $0.049 *$ \\
\hline H11 & $0.650(2)$ & $0.6962(17)$ & $0.5161(6)$ & $0.049 *$ \\
\hline $\mathrm{C} 2$ & $0.52299(18)$ & $0.59306(15)$ & $0.55418(6)$ & $0.0401(4)$ \\
\hline $\mathrm{H} 2 \mathrm{~A}$ & 0.4642 & 0.5684 & 0.5294 & $0.048 *$ \\
\hline $\mathrm{H} 2 \mathrm{~B}$ & 0.4739 & 0.6577 & 0.5690 & $0.048 *$ \\
\hline C3 & $0.54099(18)$ & $0.49095(14)$ & $0.58566(5)$ & $0.0368(4)$ \\
\hline $\mathrm{H} 3 \mathrm{~A}$ & 0.4473 & 0.4669 & 0.5967 & $0.044^{*}$ \\
\hline $\mathrm{H} 3 \mathrm{~B}$ & 0.5831 & 0.4242 & 0.5702 & $0.044 *$ \\
\hline N4 & $0.63342(14)$ & $0.52403(11)$ & $0.62269(4)$ & $0.0337(3)$ \\
\hline $\mathrm{C} 5$ & $0.77180(19)$ & $0.57192(16)$ & $0.60810(6)$ & $0.0440(4)$ \\
\hline $\mathrm{H} 5 \mathrm{~A}$ & 0.8273 & 0.5094 & 0.5942 & $0.053 *$ \\
\hline H5B & 0.8255 & 0.5995 & 0.6336 & $0.053 *$ \\
\hline C6 & $0.7534(2)$ & $0.67296(16)$ & $0.57600(6)$ & $0.0479(5)$ \\
\hline H6A & 0.7070 & 0.7391 & 0.5907 & $0.058 *$ \\
\hline H6B & 0.8470 & 0.6990 & 0.5656 & $0.058 *$ \\
\hline $\mathrm{C} 21$ & $0.63956(17)$ & $0.44215(13)$ & $0.65799(5)$ & $0.0317(3)$ \\
\hline $\mathrm{C} 22$ & $0.5429(2)$ & $0.35039(16)$ & $0.66255(6)$ & $0.0449(4)$ \\
\hline $\mathrm{H} 22$ & 0.4750 & 0.3379 & 0.6405 & $0.054 *$ \\
\hline $\mathrm{C} 23$ & $0.5436(2)$ & $0.27603(15)$ & $0.69898(6)$ & $0.0464(5)$ \\
\hline $\mathrm{H} 23$ & 0.4759 & 0.2155 & 0.7010 & $0.056^{*}$ \\
\hline $\mathrm{C} 24$ & 0.64245 (19) & $0.29057(14)$ & $0.73195(5)$ & $0.0391(4)$ \\
\hline $\mathrm{C} 25$ & $0.7405(2)$ & $0.38118(18)$ & $0.72806(6)$ & $0.0558(5)$ \\
\hline $\mathrm{H} 25$ & 0.8087 & 0.3926 & 0.7501 & $0.067 *$ \\
\hline $\mathrm{C} 26$ & $0.7395(2)$ & $0.45523(17)$ & $0.69203(6)$ & $0.0523(5)$ \\
\hline H26 & 0.8073 & 0.5157 & 0.6903 & $0.063 *$ \\
\hline $\mathrm{O} 24$ & $0.65388(16)$ & $0.22083(12)$ & $0.76910(4)$ & $0.0551(4)$ \\
\hline $\mathrm{C} 27$ & $0.5378(3)$ & $0.14351(19)$ & $0.77845(7)$ & $0.0632(6)$ \\
\hline H27A & 0.5581 & 0.0999 & 0.8049 & $0.095^{*}$ \\
\hline H27B & 0.4512 & 0.1888 & 0.7824 & $0.095^{*}$ \\
\hline $\mathrm{H} 27 \mathrm{C}$ & 0.5252 & 0.0893 & 0.7544 & $0.095 *$ \\
\hline N31 & $0.6143(2)$ & $1.14730(14)$ & $0.39733(6)$ & $0.0587(5)$ \\
\hline C32 & 0.6210 & $1.07950(15)$ & $0.43309(6)$ & $0.0439(4)$ \\
\hline H32 & 0.6092 & 1.1158 & 0.4604 & $0.053 *$ \\
\hline C33 & $0.64434(17)$ & $0.95882(13)$ & $0.43243(5)$ & $0.0332(4)$ \\
\hline C34 & $0.66384(19)$ & $0.90618(16)$ & $0.39174(6)$ & $0.0419(4)$ \\
\hline H34 & 0.6786 & 0.8249 & 0.3896 & $0.050 *$ \\
\hline C35 & $0.6611(2)$ & 0.97549 (19) & $0.35436(6)$ & $0.0517(5)$ \\
\hline H35 & 0.6767 & 0.9422 & 0.3267 & $0.062 *$ \\
\hline C36 & $0.6353(2)$ & $1.09376(19)$ & $0.35863(7)$ & $0.0588(6)$ \\
\hline
\end{tabular}




\begin{tabular}{lllll}
$\mathrm{H} 36$ & 0.6321 & 1.1397 & 0.3332 & $0.071^{*}$ \\
$\mathrm{C} 37$ & $0.64844(18)$ & $0.88736(15)$ & $0.47434(6)$ & $0.0378(4)$ \\
O31 & $0.60454(17)$ & $0.78319(11)$ & $0.47188(4)$ & $0.0589(4)$ \\
O32 & $0.69492(15)$ & $0.93503(12)$ & $0.50849(4)$ & $0.0543(4)$ \\
\hline
\end{tabular}

Atomic displacement parameters $\left(\AA^{2}\right)$

\begin{tabular}{lllllll}
\hline & $U^{11}$ & $U^{22}$ & $U^{33}$ & $U^{12}$ & $U^{13}$ & $U^{23}$ \\
\hline $\mathrm{N} 1$ & $0.0484(9)$ & $0.0353(7)$ & $0.0379(8)$ & $0.0052(7)$ & $0.0025(7)$ & $0.0083(6)$ \\
$\mathrm{C} 2$ & $0.0387(9)$ & $0.0413(9)$ & $0.0403(9)$ & $0.0052(7)$ & $-0.0030(7)$ & $0.0026(8)$ \\
$\mathrm{C} 3$ & $0.0337(9)$ & $0.0384(9)$ & $0.0383(9)$ & $-0.0016(7)$ & $-0.0021(7)$ & $0.0027(7)$ \\
$\mathrm{N} 4$ & $0.0313(7)$ & $0.0354(7)$ & $0.0344(7)$ & $-0.0020(6)$ & $0.0001(6)$ & $0.0032(6)$ \\
$\mathrm{C} 5$ & $0.0382(9)$ & $0.0509(10)$ & $0.0428(9)$ & $-0.0112(8)$ & $-0.0039(8)$ & $0.0099(8)$ \\
$\mathrm{C} 6$ & $0.0515(11)$ & $0.0435(10)$ & $0.0488(11)$ & $-0.0119(8)$ & $-0.0021(9)$ & $0.0082(8)$ \\
$\mathrm{C} 21$ & $0.0315(8)$ & $0.0321(8)$ & $0.0314(8)$ & $0.0024(6)$ & $0.0031(6)$ & $-0.0009(6)$ \\
$\mathrm{C} 22$ & $0.0483(11)$ & $0.0452(10)$ & $0.0413(10)$ & $-0.0135(8)$ & $-0.0089(8)$ & $0.0042(8)$ \\
C23 & $0.0536(11)$ & $0.0405(9)$ & $0.0452(10)$ & $-0.0153(8)$ & $0.0004(8)$ & $0.0044(8)$ \\
C24 & $0.0465(10)$ & $0.0363(8)$ & $0.0345(8)$ & $0.0008(8)$ & $0.0049(7)$ & $0.0038(7)$ \\
C25 & $0.0547(12)$ & $0.0632(12)$ & $0.0495(11)$ & $-0.0151(10)$ & $-0.0169(9)$ & $0.0159(10)$ \\
C26 & $0.0487(11)$ & $0.0556(11)$ & $0.0526(11)$ & $-0.0201(9)$ & $-0.0122(9)$ & $0.0165(9)$ \\
O24 & $0.0657(9)$ & $0.0559(8)$ & $0.0436(7)$ & $-0.0092(7)$ & $-0.0005(6)$ & $0.0169(6)$ \\
C27 & $0.0701(14)$ & $0.0575(12)$ & $0.0620(13)$ & $-0.0050(11)$ & $0.0117(11)$ & $0.0232(10)$ \\
N31 & $0.0794(13)$ & $0.0393(8)$ & $0.0573(10)$ & $-0.0007(9)$ & $-0.0084(9)$ & $0.0127(8)$ \\
C32 & $0.0537(11)$ & $0.0356(9)$ & $0.0425(10)$ & $0.0018(8)$ & $-0.0021(8)$ & $0.0007(8)$ \\
C33 & $0.0283(8)$ & $0.0318(8)$ & $0.0396(9)$ & $-0.0024(6)$ & $-0.0031(7)$ & $0.0036(6)$ \\
C34 & $0.0434(10)$ & $0.0364(9)$ & $0.0458(10)$ & $0.0001(8)$ & $-0.0040(8)$ & $-0.0040(8)$ \\
C35 & $0.0523(12)$ & $0.0664(13)$ & $0.0363(10)$ & $-0.0086(10)$ & $-0.0004(8)$ & $-0.0028(9)$ \\
C36 & $0.0712(14)$ & $0.0595(13)$ & $0.0458(11)$ & $-0.0115(11)$ & $-0.0079(10)$ & $0.0195(10)$ \\
C37 & $0.0343(9)$ & $0.0377(9)$ & $0.0415(9)$ & $-0.0008(7)$ & $-0.0032(7)$ & $0.0065(7)$ \\
O31 & $0.0833(11)$ & $0.0368(7)$ & $0.0566(8)$ & $-0.0120(7)$ & $-0.0157(7)$ & $0.0144(6)$ \\
O32 & $0.0670(9)$ & $0.0575(8)$ & $0.0385(7)$ & $-0.0166(7)$ & $-0.0106(6)$ & $0.0060(6)$ \\
& & & & & & \\
& & & & & &
\end{tabular}

Geometric parameters $\left(A,{ }^{\circ}\right)$

\begin{tabular}{llll}
\hline $\mathrm{N} 1-\mathrm{C} 2$ & $1.477(2)$ & $\mathrm{C} 24-\mathrm{C} 25$ & $1.375(3)$ \\
$\mathrm{N} 1-\mathrm{C} 6$ & $1.480(2)$ & $\mathrm{C} 24-\mathrm{O} 24$ & $1.3781(19)$ \\
$\mathrm{N} 1-\mathrm{H} 12$ & $0.94(2)$ & $\mathrm{C} 25-\mathrm{C} 26$ & $1.375(2)$ \\
$\mathrm{N} 1-\mathrm{H} 11$ & $0.97(2)$ & $\mathrm{C} 25-\mathrm{H} 25$ & 0.9300 \\
$\mathrm{C} 2-\mathrm{C} 3$ & $1.506(2)$ & $\mathrm{C} 26-\mathrm{H} 26$ & 0.9300 \\
$\mathrm{C} 2-\mathrm{H} 2 \mathrm{~A}$ & 0.9700 & $\mathrm{O} 24-\mathrm{C} 27$ & $1.416(2)$ \\
$\mathrm{C} 2-\mathrm{H} 2 \mathrm{~B}$ & 0.9700 & $\mathrm{C} 27-\mathrm{H} 27 \mathrm{~A}$ & 0.9600 \\
$\mathrm{C} 3-\mathrm{N} 4$ & $1.461(2)$ & $\mathrm{C} 27-\mathrm{H} 27 \mathrm{~B}$ & 0.9600 \\
$\mathrm{C} 3-\mathrm{H} 3 \mathrm{~A}$ & 0.9700 & $\mathrm{C} 27-\mathrm{H} 27 \mathrm{C}$ & 0.9600 \\
$\mathrm{C} 3-\mathrm{H} 3 \mathrm{~B}$ & 0.9700 & $\mathrm{~N} 31-\mathrm{C} 32$ & $1.328(2)$ \\
$\mathrm{N} 4-\mathrm{C} 21$ & $1.4152(19)$ & $\mathrm{N} 31-\mathrm{C} 36$ & $1.334(3)$ \\
$\mathrm{N} 4-\mathrm{C} 5$ & $1.462(2)$ & $\mathrm{C} 32-\mathrm{C} 33$ & $1.380(2)$ \\
$\mathrm{C} 5-\mathrm{C} 6$ & $1.509(2)$ & $\mathrm{C} 32-\mathrm{H} 32$ & 0.9300 \\
$\mathrm{C} 5-\mathrm{H} 5 \mathrm{~A}$ & 0.9700 & $\mathrm{C} 33-\mathrm{C} 34$ & $1.381(2)$
\end{tabular}




\begin{tabular}{|c|c|c|c|}
\hline $\mathrm{C} 5-\mathrm{H} 5 \mathrm{~B}$ & 0.9700 & $\mathrm{C} 33-\mathrm{C} 37$ & $1.505(2)$ \\
\hline C6-H6A & 0.9700 & $\mathrm{C} 34-\mathrm{C} 35$ & $1.377(3)$ \\
\hline C6-H6B & 0.9700 & C34-H34 & 0.9300 \\
\hline $\mathrm{C} 21-\mathrm{C} 22$ & $1.377(2)$ & $\mathrm{C} 35-\mathrm{C} 36$ & $1.363(3)$ \\
\hline $\mathrm{C} 21-\mathrm{C} 26$ & $1.395(2)$ & C35-H35 & 0.9300 \\
\hline $\mathrm{C} 22-\mathrm{C} 23$ & $1.387(2)$ & $\mathrm{C} 36-\mathrm{H} 36$ & 0.9300 \\
\hline $\mathrm{C} 22-\mathrm{H} 22$ & 0.9300 & $\mathrm{C} 37-\mathrm{O} 32$ & $1.244(2)$ \\
\hline $\mathrm{C} 23-\mathrm{C} 24$ & $1.366(3)$ & $\mathrm{C} 37-\mathrm{O} 31$ & $1.247(2)$ \\
\hline $\mathrm{C} 23-\mathrm{H} 23$ & 0.9300 & & \\
\hline $\mathrm{C} 2-\mathrm{N} 1-\mathrm{C} 6$ & $109.37(13)$ & $\mathrm{C} 24-\mathrm{C} 23-\mathrm{C} 22$ & $120.82(16)$ \\
\hline $\mathrm{C} 2-\mathrm{N} 1-\mathrm{H} 12$ & $108.5(12)$ & $\mathrm{C} 24-\mathrm{C} 23-\mathrm{H} 23$ & 119.6 \\
\hline $\mathrm{C} 6-\mathrm{N} 1-\mathrm{H} 12$ & $108.6(12)$ & $\mathrm{C} 22-\mathrm{C} 23-\mathrm{H} 23$ & 119.6 \\
\hline $\mathrm{C} 2-\mathrm{N} 1-\mathrm{H} 11$ & $109.1(11)$ & $\mathrm{C} 23-\mathrm{C} 24-\mathrm{C} 25$ & $118.14(16)$ \\
\hline $\mathrm{C} 6-\mathrm{N} 1-\mathrm{H} 11$ & $113.2(11)$ & $\mathrm{C} 23-\mathrm{C} 24-\mathrm{O} 24$ & $125.48(16)$ \\
\hline $\mathrm{H} 12-\mathrm{N} 1-\mathrm{H} 11$ & $107.9(15)$ & $\mathrm{C} 25-\mathrm{C} 24-\mathrm{O} 24$ & $116.38(16)$ \\
\hline $\mathrm{N} 1-\mathrm{C} 2-\mathrm{C} 3$ & $110.55(13)$ & $\mathrm{C} 24-\mathrm{C} 25-\mathrm{C} 26$ & $121.05(17)$ \\
\hline $\mathrm{N} 1-\mathrm{C} 2-\mathrm{H} 2 \mathrm{~A}$ & 109.5 & $\mathrm{C} 24-\mathrm{C} 25-\mathrm{H} 25$ & 119.5 \\
\hline $\mathrm{C} 3-\mathrm{C} 2-\mathrm{H} 2 \mathrm{~A}$ & 109.5 & $\mathrm{C} 26-\mathrm{C} 25-\mathrm{H} 25$ & 119.5 \\
\hline $\mathrm{N} 1-\mathrm{C} 2-\mathrm{H} 2 \mathrm{~B}$ & 109.5 & $\mathrm{C} 25-\mathrm{C} 26-\mathrm{C} 21$ & $121.82(17)$ \\
\hline $\mathrm{C} 3-\mathrm{C} 2-\mathrm{H} 2 \mathrm{~B}$ & 109.5 & $\mathrm{C} 25-\mathrm{C} 26-\mathrm{H} 26$ & 119.1 \\
\hline $\mathrm{H} 2 \mathrm{~A}-\mathrm{C} 2-\mathrm{H} 2 \mathrm{~B}$ & 108.1 & $\mathrm{C} 21-\mathrm{C} 26-\mathrm{H} 26$ & 119.1 \\
\hline $\mathrm{N} 4-\mathrm{C} 3-\mathrm{C} 2$ & $110.87(13)$ & $\mathrm{C} 24-\mathrm{O} 24-\mathrm{C} 27$ & $117.22(15)$ \\
\hline $\mathrm{N} 4-\mathrm{C} 3-\mathrm{H} 3 \mathrm{~A}$ & 109.5 & $\mathrm{O} 24-\mathrm{C} 27-\mathrm{H} 27 \mathrm{~A}$ & 109.5 \\
\hline $\mathrm{C} 2-\mathrm{C} 3-\mathrm{H} 3 \mathrm{~A}$ & 109.5 & $\mathrm{O} 24-\mathrm{C} 27-\mathrm{H} 27 \mathrm{~B}$ & 109.5 \\
\hline $\mathrm{N} 4-\mathrm{C} 3-\mathrm{H} 3 \mathrm{~B}$ & 109.5 & $\mathrm{H} 27 \mathrm{~A}-\mathrm{C} 27-\mathrm{H} 27 \mathrm{~B}$ & 109.5 \\
\hline $\mathrm{C} 2-\mathrm{C} 3-\mathrm{H} 3 \mathrm{~B}$ & 109.5 & $\mathrm{O} 24-\mathrm{C} 27-\mathrm{H} 27 \mathrm{C}$ & 109.5 \\
\hline $\mathrm{H} 3 \mathrm{~A}-\mathrm{C} 3-\mathrm{H} 3 \mathrm{~B}$ & 108.1 & $\mathrm{H} 27 \mathrm{~A}-\mathrm{C} 27-\mathrm{H} 27 \mathrm{C}$ & 109.5 \\
\hline $\mathrm{C} 21-\mathrm{N} 4-\mathrm{C} 3$ & $115.94(13)$ & $\mathrm{H} 27 \mathrm{~B}-\mathrm{C} 27-\mathrm{H} 27 \mathrm{C}$ & 109.5 \\
\hline $\mathrm{C} 21-\mathrm{N} 4-\mathrm{C} 5$ & $115.77(13)$ & $\mathrm{C} 32-\mathrm{N} 31-\mathrm{C} 36$ & $116.71(16)$ \\
\hline $\mathrm{C} 3-\mathrm{N} 4-\mathrm{C} 5$ & $112.21(13)$ & $\mathrm{N} 31-\mathrm{C} 32-\mathrm{C} 33$ & $124.38(17)$ \\
\hline $\mathrm{N} 4-\mathrm{C} 5-\mathrm{C} 6$ & $112.02(15)$ & $\mathrm{N} 31-\mathrm{C} 32-\mathrm{H} 32$ & 117.8 \\
\hline $\mathrm{N} 4-\mathrm{C} 5-\mathrm{H} 5 \mathrm{~A}$ & 109.2 & $\mathrm{C} 33-\mathrm{C} 32-\mathrm{H} 32$ & 117.8 \\
\hline $\mathrm{C} 6-\mathrm{C} 5-\mathrm{H} 5 \mathrm{~A}$ & 109.2 & $\mathrm{C} 32-\mathrm{C} 33-\mathrm{C} 34$ & $117.29(16)$ \\
\hline $\mathrm{N} 4-\mathrm{C} 5-\mathrm{H} 5 \mathrm{~B}$ & 109.2 & $\mathrm{C} 32-\mathrm{C} 33-\mathrm{C} 37$ & $121.41(15)$ \\
\hline $\mathrm{C} 6-\mathrm{C} 5-\mathrm{H} 5 \mathrm{~B}$ & 109.2 & $\mathrm{C} 34-\mathrm{C} 33-\mathrm{C} 37$ & $121.31(14)$ \\
\hline $\mathrm{H} 5 \mathrm{~A}-\mathrm{C} 5-\mathrm{H} 5 \mathrm{~B}$ & 107.9 & $\mathrm{C} 35-\mathrm{C} 34-\mathrm{C} 33$ & $119.20(17)$ \\
\hline $\mathrm{N} 1-\mathrm{C} 6-\mathrm{C} 5$ & $109.88(14)$ & $\mathrm{C} 35-\mathrm{C} 34-\mathrm{H} 34$ & 120.4 \\
\hline $\mathrm{N} 1-\mathrm{C} 6-\mathrm{H} 6 \mathrm{~A}$ & 109.7 & $\mathrm{C} 33-\mathrm{C} 34-\mathrm{H} 34$ & 120.4 \\
\hline $\mathrm{C} 5-\mathrm{C} 6-\mathrm{H} 6 \mathrm{~A}$ & 109.7 & $\mathrm{C} 36-\mathrm{C} 35-\mathrm{C} 34$ & $118.80(18)$ \\
\hline $\mathrm{N} 1-\mathrm{C} 6-\mathrm{H} 6 \mathrm{~B}$ & 109.7 & $\mathrm{C} 36-\mathrm{C} 35-\mathrm{H} 35$ & 120.6 \\
\hline $\mathrm{C} 5-\mathrm{C} 6-\mathrm{H} 6 \mathrm{~B}$ & 109.7 & $\mathrm{C} 34-\mathrm{C} 35-\mathrm{H} 35$ & 120.6 \\
\hline $\mathrm{H} 6 \mathrm{~A}-\mathrm{C} 6-\mathrm{H} 6 \mathrm{~B}$ & 108.2 & $\mathrm{~N} 31-\mathrm{C} 36-\mathrm{C} 35$ & $123.59(18)$ \\
\hline $\mathrm{C} 22-\mathrm{C} 21-\mathrm{C} 26$ & $116.02(15)$ & $\mathrm{N} 31-\mathrm{C} 36-\mathrm{H} 36$ & 118.2 \\
\hline $\mathrm{C} 22-\mathrm{C} 21-\mathrm{N} 4$ & $122.74(15)$ & $\mathrm{C} 35-\mathrm{C} 36-\mathrm{H} 36$ & 118.2 \\
\hline $\mathrm{C} 26-\mathrm{C} 21-\mathrm{N} 4$ & $121.11(14)$ & $\mathrm{O} 32-\mathrm{C} 37-\mathrm{O} 31$ & $124.84(16)$ \\
\hline $\mathrm{C} 21-\mathrm{C} 22-\mathrm{C} 23$ & $122.14(16)$ & $\mathrm{O} 32-\mathrm{C} 37-\mathrm{C} 33$ & $118.62(15)$ \\
\hline $\mathrm{C} 21-\mathrm{C} 22-\mathrm{H} 22$ & 118.9 & $\mathrm{O} 31-\mathrm{C} 37-\mathrm{C} 33$ & $116.54(15)$ \\
\hline
\end{tabular}




$\begin{array}{ll}\mathrm{C} 23-\mathrm{C} 22-\mathrm{H} 22 & 118.9 \\ \mathrm{C} 6-\mathrm{N} 1-\mathrm{C} 2-\mathrm{C} 3 & -59.85(18) \\ \mathrm{N} 1-\mathrm{C} 2-\mathrm{C} 3-\mathrm{N} 4 & 57.18(18) \\ \mathrm{C} 2-\mathrm{C} 3-\mathrm{N} 4-\mathrm{C} 21 & 170.15(13) \\ \mathrm{C} 2-\mathrm{C} 3-\mathrm{N} 4-\mathrm{C} 5 & -53.72(18) \\ \mathrm{C} 21-\mathrm{N} 4-\mathrm{C} 5-\mathrm{C} 6 & -170.28(14) \\ \mathrm{C} 3-\mathrm{N} 4-\mathrm{C} 5-\mathrm{C} 6 & 53.52(19) \\ \mathrm{C} 2-\mathrm{N} 1-\mathrm{C} 6-\mathrm{C} 5 & 58.59(19) \\ \mathrm{N} 4-\mathrm{C} 5-\mathrm{C} 6-\mathrm{N} 1 & -55.7(2) \\ \mathrm{C} 3-\mathrm{N} 4-\mathrm{C} 21-\mathrm{C} 22 & -13.9(2) \\ \mathrm{C} 5-\mathrm{N} 4-\mathrm{C} 21-\mathrm{C} 22 & -148.45(17) \\ \mathrm{C} 3-\mathrm{N} 4-\mathrm{C} 21-\mathrm{C} 26 & 170.48(16) \\ \mathrm{C} 5-\mathrm{N} 4-\mathrm{C} 21-\mathrm{C} 26 & 35.9(2) \\ \mathrm{C} 26-\mathrm{C} 21-\mathrm{C} 22-\mathrm{C} 23 & 0.7(3) \\ \mathrm{N} 4-\mathrm{C} 21-\mathrm{C} 22-\mathrm{C} 23 & -175.17(16) \\ \mathrm{C} 21-\mathrm{C} 22-\mathrm{C} 23-\mathrm{C} 24 & -0.6(3) \\ \mathrm{C} 22-\mathrm{C} 23-\mathrm{C} 24-\mathrm{C} 25 & 0.3(3) \\ \mathrm{C} 22-\mathrm{C} 23-\mathrm{C} 24-\mathrm{O} 24 & -178.99(17) \\ \mathrm{C} 23-\mathrm{C} 24-\mathrm{C} 25-\mathrm{C} 26 & 0.0(3) \\ \end{array}$

$$
\begin{aligned}
& \mathrm{O} 24-\mathrm{C} 24-\mathrm{C} 25-\mathrm{C} 26 \\
& \mathrm{C} 24-\mathrm{C} 25-\mathrm{C} 26-\mathrm{C} 21 \\
& \mathrm{C} 22-\mathrm{C} 21-\mathrm{C} 26-\mathrm{C} 25 \\
& \mathrm{~N} 4-\mathrm{C} 21-\mathrm{C} 26-\mathrm{C} 25 \\
& \mathrm{C} 23-\mathrm{C} 24-\mathrm{O} 24-\mathrm{C} 27 \\
& \mathrm{C} 25-\mathrm{C} 24-\mathrm{O} 24-\mathrm{C} 27 \\
& \mathrm{C} 36-\mathrm{N} 31-\mathrm{C} 32-\mathrm{C} 33 \\
& \mathrm{~N} 31-\mathrm{C} 32-\mathrm{C} 33-\mathrm{C} 34 \\
& \mathrm{~N} 31-\mathrm{C} 32-\mathrm{C} 33-\mathrm{C} 37 \\
& \mathrm{C} 32-\mathrm{C} 33-\mathrm{C} 34-\mathrm{C} 35 \\
& \mathrm{C} 37-\mathrm{C} 33-\mathrm{C} 34-\mathrm{C} 35 \\
& \mathrm{C} 33-\mathrm{C} 34-\mathrm{C} 35-\mathrm{C} 36 \\
& \mathrm{C} 32-\mathrm{N} 31-\mathrm{C} 36-\mathrm{C} 35 \\
& \mathrm{C} 34-\mathrm{C} 35-\mathrm{C} 36-\mathrm{N} 31 \\
& \mathrm{C} 32-\mathrm{C} 33-\mathrm{C} 37-\mathrm{O} 32 \\
& \mathrm{C} 34-\mathrm{C} 33-\mathrm{C} 37-\mathrm{O} 32 \\
& \mathrm{C} 32-\mathrm{C} 33-\mathrm{C} 37-\mathrm{O} 31 \\
& \mathrm{C} 34-\mathrm{C} 33-\mathrm{C} 37-\mathrm{O} 31
\end{aligned}
$$

$$
\begin{aligned}
& 179.32(18) \\
& 0.1(3) \\
& -0.4(3) \\
& 175.52(18) \\
& -13.4(3) \\
& 167.31(18) \\
& -1.7(3) \\
& 0.9(3) \\
& -179.08(17) \\
& 0.9(3) \\
& -179.12(16) \\
& -1.8(3) \\
& 0.7(3) \\
& 1.0(3) \\
& -32.1(2) \\
& 147.86(17) \\
& 148.09(18) \\
& -31.9(2)
\end{aligned}
$$

Hydrogen-bond geometry $\left(\AA,{ }^{\circ}\right)$

\begin{tabular}{lllll}
\hline$D-\mathrm{H} \cdots A$ & $D-\mathrm{H}$ & $\mathrm{H} \cdots A$ & $D \cdots A$ & $D-\mathrm{H} \cdots A$ \\
\hline $\mathrm{N} 1-\mathrm{H} 11 \cdots \mathrm{O} 31$ & $0.976(19)$ & $1.714(19)$ & $2.677(2)$ & $168.2(18)$ \\
$\mathrm{N} 1-\mathrm{H} 12 \cdots \mathrm{O} 32^{\mathrm{i}}$ & $0.94(2)$ & $1.82(2)$ & $2.749(2)$ & $168.3(17)$ \\
$\mathrm{C} 2-\mathrm{H} 2 B \cdots \mathrm{N} 31^{\mathrm{ii}}$ & 0.97 & 2.56 & $3.518(2)$ & 169 \\
$\mathrm{C} 36-\mathrm{H} 36 \cdots \mathrm{O} 24^{\mathrm{iii}}$ & 0.93 & 2.51 & $3.432(2)$ & 172 \\
$\mathrm{C} 3-\mathrm{H} 3 A \cdots C g 1^{\mathrm{iv}}$ & 0.97 & 2.97 & $3.775(2)$ & 156 \\
\hline
\end{tabular}

Symmetry codes: (i) $-x+3 / 2, y-1 / 2, z$; (ii) $-x+1,-y+2,-z+1$; (iii) $x,-y+3 / 2, z-1 / 2$; (iv) $x-1 / 2,-y+3 / 2,-z+1$.

4-(4-Methoxyphenyl)piperazin-1-ium 2-hydroxy-3,5-dinitrobenzoate (VII)

Crystal data

$\mathrm{C}_{7} \mathrm{H}_{3} \mathrm{~N}_{2} \mathrm{O}_{7}^{+} \cdot \mathrm{C}_{11} \mathrm{H}_{17} \mathrm{~N}_{2} \mathrm{O}^{-}$

$M_{r}=420.38$

Monoclinic, $P 2{ }_{1} / c$

$a=7.5500(9) \AA$

$b=7.6489(9) \AA$

$c=32.719(6) \AA$

$\beta=91.30(1)^{\circ}$

$V=1889.0(5) \AA^{3}$

$Z=4$

\section{Data collection}

Oxford Diffraction Xcalibur with Sapphire CCD diffractometer

Radiation source: Enhance (Mo) X-ray Source Graphite monochromator $\omega$ scans
$F(000)=880$

$D_{\mathrm{x}}=1.478 \mathrm{Mg} \mathrm{m}^{-3}$

Mo $K \alpha$ radiation, $\lambda=0.71073 \AA$

Cell parameters from 4078 reflections

$\theta=2.7-28.0^{\circ}$

$\mu=0.12 \mathrm{~mm}^{-1}$

$T=296 \mathrm{~K}$

Block, colourless

$0.18 \times 0.12 \times 0.06 \mathrm{~mm}$

Absorption correction: multi-scan

(CrysAlis RED; Oxford Diffraction, 2009)

$T_{\min }=0.916, T_{\max }=0.993$

8215 measured reflections

4074 independent reflections 
2003 reflections with $I>2 \sigma(I)$

$R_{\text {int }}=0.038$

$\theta_{\max }=28.0^{\circ}, \theta_{\min }=2.7^{\circ}$

\section{Refinement}

Refinement on $F^{2}$

Least-squares matrix: full

$R\left[F^{2}>2 \sigma\left(F^{2}\right)\right]=0.066$

$w R\left(F^{2}\right)=0.128$

$S=1.03$

4074 reflections

281 parameters

0 restraints

$$
\begin{aligned}
& h=-9 \rightarrow 5 \\
& k=-9 \rightarrow 9 \\
& l=-42 \rightarrow 41
\end{aligned}
$$

Primary atom site location: difference Fourier map

Hydrogen site location: mixed

$\mathrm{H}$ atoms treated by a mixture of independent and constrained refinement

$w=1 /\left[\sigma^{2}\left(F_{\mathrm{o}}^{2}\right)+(0.0413 P)^{2}+0.4471 P\right]$ where $P=\left(F_{\mathrm{o}}{ }^{2}+2 F_{\mathrm{c}}{ }^{2}\right) / 3$

$(\Delta / \sigma)_{\max }<0.001$

$\Delta \rho_{\max }=0.22 \mathrm{e} \AA^{-3}$

$\Delta \rho_{\min }=-0.23$ e $\AA^{-3}$

Special details

Experimental. Compound (VII). IR ( $\left.\mathrm{KBr}, \mathrm{cm}^{-1}\right) 3084\left(\mathrm{NH}_{2}\right), 2834\left(\mathrm{OCH}_{3}\right), 1568(\mathrm{COO}), 1499\left(\mathrm{NO}_{2}\right)$. NMR $\left(\mathrm{CDCl}_{3}\right)$ $\delta\left({ }^{1} \mathrm{H}\right) 3.05\left(\mathrm{~m}, 4 \mathrm{H}\right.$, piperazine), $3.37\left(\mathrm{~m}, 4 \mathrm{H}\right.$, piperazine), $3.77\left(\mathrm{~s}, 3 \mathrm{H}, \mathrm{OCH}_{3}\right), 6.85(\mathrm{~m}, 4 \mathrm{H}$, methoxyphenyl), $7.52(\mathrm{~s}, 1 \mathrm{H}$, 3,5-dinitrosalicylate), 8.09 (s, 1H, 3,5-dinitrosalicylate), 8.99 (s, 1H, 3,5-dinitrosalicylate).

Geometry. All esds (except the esd in the dihedral angle between two 1.s. planes) are estimated using the full covariance matrix. The cell esds are taken into account individually in the estimation of esds in distances, angles and torsion angles; correlations between esds in cell parameters are only used when they are defined by crystal symmetry. An approximate (isotropic) treatment of cell esds is used for estimating esds involving l.s. planes.

Fractional atomic coordinates and isotropic or equivalent isotropic displacement parameters $\left(\hat{A}^{2}\right)$

\begin{tabular}{lllll}
\hline & $x$ & $y$ & $z$ & $U_{\text {iso }} / U_{\text {eq }}$ \\
\hline $\mathrm{N} 1$ & $0.6215(3)$ & $0.5723(4)$ & $0.61103(8)$ & $0.0465(7)$ \\
$\mathrm{H} 11$ & $0.703(4)$ & $0.587(4)$ & $0.5906(9)$ & $0.056^{*}$ \\
$\mathrm{H} 12$ & $0.533(4)$ & $0.494(4)$ & $0.6028(9)$ & $0.056^{*}$ \\
$\mathrm{C} 2$ & $0.5349(4)$ & $0.7433(4)$ & $0.61776(9)$ & $0.0531(9)$ \\
$\mathrm{H} 2 \mathrm{~A}$ & 0.4783 & 0.7826 & 0.5925 & $0.064^{*}$ \\
$\mathrm{H} 2 \mathrm{~B}$ & 0.6235 & 0.8292 & 0.6258 & $0.064^{*}$ \\
$\mathrm{C} 3$ & $0.3982(4)$ & $0.7294(4)$ & $0.65055(9)$ & $0.0489(8)$ \\
$\mathrm{H} 3 \mathrm{~A}$ & 0.3504 & 0.8446 & 0.6559 & $0.059^{*}$ \\
$\mathrm{H} 3 \mathrm{~B}$ & 0.3016 & 0.6558 & 0.6407 & $0.059^{*}$ \\
$\mathrm{~N} 4$ & $0.4712(3)$ & $0.6567(3)$ & $0.68854(7)$ & $0.0399(6)$ \\
$\mathrm{C} 5$ & $0.5669(4)$ & $0.4924(4)$ & $0.68187(9)$ & $0.0483(8)$ \\
$\mathrm{H} 5 \mathrm{~A}$ & 0.4832 & 0.4024 & 0.6734 & $0.058^{*}$ \\
$\mathrm{H} 5 \mathrm{~B}$ & 0.6233 & 0.4554 & 0.7074 & $0.058^{*}$ \\
$\mathrm{C} 6$ & $0.7057(4)$ & $0.5118(4)$ & $0.64972(8)$ & $0.0505(8)$ \\
$\mathrm{H} 6 \mathrm{~A}$ & 0.7947 & 0.5955 & 0.6588 & $0.061^{*}$ \\
$\mathrm{H} 6 \mathrm{~B}$ & 0.7637 & 0.4003 & 0.6454 & $0.061^{*}$ \\
$\mathrm{C} 21$ & $0.3501(4)$ & $0.6515(4)$ & $0.72152(9)$ & $0.0382(7)$ \\
$\mathrm{C} 22$ & $0.1878(4)$ & $0.7370(4)$ & $0.72002(10)$ & $0.0510(8)$ \\
$\mathrm{H} 22$ & 0.1538 & 0.7966 & 0.6963 & $0.061^{*}$ \\
$\mathrm{C} 23$ & $0.0745(4)$ & $0.7358(4)$ & $0.75303(10)$ & $0.0522(8)$ \\
H23 & -0.0327 & 0.7954 & 0.7512 & $0.063^{*}$ \\
$\mathrm{C} 24$ & $0.1201(4)$ & $0.6473(4)$ & $0.78814(9)$ & $0.0455(8)$ \\
$\mathrm{C} 25$ & $0.2807(4)$ & $0.5624(4)$ & $0.79002(9)$ & $0.0547(9)$ \\
& & & &
\end{tabular}




\begin{tabular}{lllll} 
H25 & 0.3138 & 0.5028 & 0.8138 & $0.066^{*}$ \\
C26 & $0.3934(4)$ & $0.5636(4)$ & $0.75758(9)$ & $0.0499(8)$ \\
H26 & 0.5008 & 0.5044 & 0.7598 & $0.060^{*}$ \\
O24 & $0.0184(3)$ & $0.6354(3)$ & $0.82246(6)$ & $0.0640(7)$ \\
C27 & $-0.1485(4)$ & $0.7203(5)$ & $0.82152(11)$ & $0.0745(1)$ \\
H27A & -0.2061 & 0.7017 & 0.8470 & $0.112^{*}$ \\
H27B & -0.2206 & 0.6733 & 0.7996 & $0.112^{*}$ \\
H27C & -0.1318 & 0.8433 & 0.8173 & $0.112^{*}$ \\
C31 & $0.8836(3)$ & $0.6893(4)$ & $0.46865(8)$ & $0.0355(7)$ \\
C32 & $0.8864(3)$ & $0.7026(4)$ & $0.51247(9)$ & $0.0374(7)$ \\
C33 & $1.0424(3)$ & $0.7812(4)$ & $0.52995(8)$ & $0.0352(7)$ \\
C34 & $1.1812(3)$ & $0.8371(4)$ & $0.50706(8)$ & $0.0372(7)$ \\
H34 & 1.2797 & 0.8892 & 0.5195 & $0.045^{*}$ \\
C35 & $1.1728(3)$ & $0.8150(4)$ & $0.46526(9)$ & $0.0368(7)$ \\
C36 & $1.0252(3)$ & $0.7425(4)$ & $0.44590(9)$ & $0.0383(7)$ \\
H36 & 1.0217 & 0.7298 & 0.4176 & $0.046^{*}$ \\
C37 & $0.7243(4)$ & $0.6189(4)$ & $0.44690(10)$ & $0.0446(8)$ \\
O31 & $0.7120(3)$ & $0.6063(3)$ & $0.40978(6)$ & $0.0609(7)$ \\
O32 & $0.5931(2)$ & $0.5689(3)$ & $0.46961(7)$ & $0.0626(7)$ \\
H32 & $0.638(4)$ & $0.590(5)$ & $0.4996(12)$ & $0.094^{*}$ \\
O33 & $0.7553(2)$ & $0.6499(3)$ & $0.53332(6)$ & $0.0530(6)$ \\
N33 & $1.0576(3)$ & $0.8102(4)$ & $0.57403(8)$ & $0.0481(7)$ \\
O34 & $0.9695(3)$ & $0.7215(4)$ & $0.59704(7)$ & $0.0868(9)$ \\
O35 & $1.1628(3)$ & $0.9203(3)$ & $0.58660(6)$ & $0.0627(7)$ \\
N35 & $1.3207(3)$ & $0.8725(3)$ & $0.44092(9)$ & $0.0492(7)$ \\
O36 & $1.4399(3)$ & $0.9552(3)$ & $0.45754(7)$ & $0.0715(7)$ \\
O37 & $1.3199(3)$ & $0.8357(3)$ & $0.40434(7)$ & $0.0687(7)$ \\
& & & & \\
\hline
\end{tabular}

Atomic displacement parameters $\left(\AA^{2}\right)$

\begin{tabular}{lllllll}
\hline & $U^{11}$ & $U^{22}$ & $U^{33}$ & $U^{12}$ & $U^{13}$ & $U^{23}$ \\
\hline $\mathrm{N} 1$ & $0.0429(15)$ & $0.061(2)$ & $0.0360(15)$ & $-0.0125(14)$ & $0.0016(11)$ & $-0.0055(14)$ \\
$\mathrm{C} 2$ & $0.064(2)$ & $0.053(2)$ & $0.0417(19)$ & $-0.0007(17)$ & $0.0004(16)$ & $0.0075(17)$ \\
$\mathrm{C} 3$ & $0.0565(19)$ & $0.053(2)$ & $0.0371(18)$ & $0.0086(16)$ & $0.0021(15)$ & $0.0053(16)$ \\
$\mathrm{N} 4$ & $0.0438(13)$ & $0.0416(16)$ & $0.0342(14)$ & $0.0040(11)$ & $-0.0003(11)$ & $0.0033(12)$ \\
$\mathrm{C} 5$ & $0.0505(18)$ & $0.055(2)$ & $0.0397(19)$ & $0.0108(16)$ & $0.0033(14)$ & $0.0063(16)$ \\
$\mathrm{C} 6$ & $0.0477(18)$ & $0.066(2)$ & $0.0374(18)$ & $0.0033(16)$ & $-0.0019(14)$ & $-0.0007(17)$ \\
$\mathrm{C} 21$ & $0.0430(16)$ & $0.0340(18)$ & $0.0375(17)$ & $-0.0001(13)$ & $-0.0035(13)$ & $-0.0012(14)$ \\
$\mathrm{C} 22$ & $0.0511(19)$ & $0.057(2)$ & $0.045(2)$ & $0.0118(16)$ & $0.0001(15)$ & $0.0135(17)$ \\
$\mathrm{C} 23$ & $0.0480(18)$ & $0.053(2)$ & $0.056(2)$ & $0.0132(15)$ & $0.0034(16)$ & $0.0039(18)$ \\
$\mathrm{C} 24$ & $0.0482(18)$ & $0.045(2)$ & $0.044(2)$ & $0.0021(15)$ & $0.0057(15)$ & $-0.0026(16)$ \\
$\mathrm{C} 25$ & $0.062(2)$ & $0.064(2)$ & $0.0382(19)$ & $0.0154(18)$ & $-0.0005(15)$ & $0.0126(18)$ \\
$\mathrm{C} 26$ & $0.0490(18)$ & $0.059(2)$ & $0.0417(19)$ & $0.0167(16)$ & $-0.0010(15)$ & $0.0055(17)$ \\
O24 & $0.0614(15)$ & $0.0814(18)$ & $0.0497(14)$ & $0.0129(12)$ & $0.0142(11)$ & $0.0028(13)$ \\
C27 & $0.057(2)$ & $0.096(3)$ & $0.071(3)$ & $0.014(2)$ & $0.0207(19)$ & $-0.007(2)$ \\
C31 & $0.0344(15)$ & $0.0352(17)$ & $0.0366(17)$ & $-0.0028(13)$ & $-0.0008(13)$ & $-0.0002(14)$ \\
C32 & $0.0318(15)$ & $0.0367(18)$ & $0.0437(18)$ & $0.0004(13)$ & $0.0011(13)$ & $0.0046(15)$ \\
C33 & $0.0367(16)$ & $0.0371(18)$ & $0.0315(16)$ & $0.0003(13)$ & $-0.0022(13)$ & $0.0003(14)$
\end{tabular}




\begin{tabular}{lllllll}
\hline C34 & $0.0352(15)$ & $0.0340(18)$ & $0.0422(18)$ & $-0.0016(13)$ & $-0.0053(13)$ & $-0.0020(15)$ \\
C35 & $0.0316(15)$ & $0.0345(17)$ & $0.0443(19)$ & $-0.0064(13)$ & $0.0036(13)$ & $0.0017(15)$ \\
C36 & $0.0405(16)$ & $0.0355(17)$ & $0.0388(17)$ & $-0.0019(13)$ & $0.0018(13)$ & $-0.0015(14)$ \\
C37 & $0.0397(17)$ & $0.048(2)$ & $0.046(2)$ & $-0.0086(14)$ & $-0.0020(15)$ & $0.0005(16)$ \\
O31 & $0.0570(13)$ & $0.0868(19)$ & $0.0389(14)$ & $-0.0254(12)$ & $-0.0012(10)$ & $-0.0093(13)$ \\
O32 & $0.0429(13)$ & $0.099(2)$ & $0.0455(13)$ & $-0.0297(12)$ & $-0.0024(10)$ & $0.0073(14)$ \\
O33 & $0.0396(12)$ & $0.0793(17)$ & $0.0404(13)$ & $-0.0153(11)$ & $0.0032(10)$ & $0.0039(12)$ \\
N33 & $0.0381(14)$ & $0.063(2)$ & $0.0424(17)$ & $0.0001(13)$ & $-0.0041(12)$ & $0.0013(15)$ \\
O34 & $0.0712(16)$ & $0.146(3)$ & $0.0427(15)$ & $-0.0443(16)$ & $0.0011(12)$ & $0.0102(16)$ \\
O35 & $0.0688(15)$ & $0.0690(17)$ & $0.0495(14)$ & $-0.0132(13)$ & $-0.0138(11)$ & $-0.0063(13)$ \\
N35 & $0.0460(16)$ & $0.0512(18)$ & $0.0505(18)$ & $-0.0110(13)$ & $0.0022(13)$ & $0.0012(15)$ \\
O36 & $0.0604(14)$ & $0.092(2)$ & $0.0616(16)$ & $-0.0425(13)$ & $0.0006(12)$ & $-0.0031(14)$ \\
O37 & $0.0643(15)$ & $0.095(2)$ & $0.0474(15)$ & $-0.0258(13)$ & $0.0132(11)$ & $-0.0123(15)$ \\
\hline
\end{tabular}

Geometric parameters $\left(\AA,{ }^{\circ}\right)$

\begin{tabular}{|c|c|c|c|}
\hline $\mathrm{N} 1-\mathrm{C} 6$ & $1.478(4)$ & $\mathrm{C} 25-\mathrm{H} 25$ & 0.9300 \\
\hline $\mathrm{N} 1-\mathrm{C} 2$ & $1.481(4)$ & $\mathrm{C} 26-\mathrm{H} 26$ & 0.9300 \\
\hline N1-H11 & $0.93(3)$ & $\mathrm{O} 24-\mathrm{C} 27$ & $1.417(3)$ \\
\hline $\mathrm{N} 1-\mathrm{H} 12$ & $0.93(3)$ & $\mathrm{C} 27-\mathrm{H} 27 \mathrm{~A}$ & 0.9600 \\
\hline $\mathrm{C} 2-\mathrm{C} 3$ & $1.509(4)$ & $\mathrm{C} 27-\mathrm{H} 27 \mathrm{~B}$ & 0.9600 \\
\hline $\mathrm{C} 2-\mathrm{H} 2 \mathrm{~A}$ & 0.9700 & $\mathrm{C} 27-\mathrm{H} 27 \mathrm{C}$ & 0.9600 \\
\hline $\mathrm{C} 2-\mathrm{H} 2 \mathrm{~B}$ & 0.9700 & $\mathrm{C} 31-\mathrm{C} 36$ & $1.378(4)$ \\
\hline $\mathrm{C} 3-\mathrm{N} 4$ & $1.458(3)$ & $\mathrm{C} 31-\mathrm{C} 32$ & $1.437(4)$ \\
\hline $\mathrm{C} 3-\mathrm{H} 3 \mathrm{~A}$ & 0.9700 & $\mathrm{C} 31-\mathrm{C} 37$ & $1.485(4)$ \\
\hline С $3-\mathrm{H} 3 \mathrm{~B}$ & 0.9700 & $\mathrm{C} 32-\mathrm{O} 33$ & $1.280(3)$ \\
\hline $\mathrm{N} 4-\mathrm{C} 21$ & $1.431(3)$ & $\mathrm{C} 32-\mathrm{C} 33$ & $1.430(4)$ \\
\hline $\mathrm{N} 4-\mathrm{C} 5$ & $1.469(3)$ & $\mathrm{C} 33-\mathrm{C} 34$ & $1.370(3)$ \\
\hline $\mathrm{C} 5-\mathrm{C} 6$ & $1.509(4)$ & $\mathrm{C} 33-\mathrm{N} 33$ & $1.461(3)$ \\
\hline $\mathrm{C} 5-\mathrm{H} 5 \mathrm{~A}$ & 0.9700 & $\mathrm{C} 34-\mathrm{C} 35$ & $1.378(4)$ \\
\hline $\mathrm{C} 5-\mathrm{H} 5 \mathrm{~B}$ & 0.9700 & C34-H34 & 0.9300 \\
\hline C6-H6A & 0.9700 & $\mathrm{C} 35-\mathrm{C} 36$ & 1.385 \\
\hline $\mathrm{C} 6-\mathrm{H} 6 \mathrm{~B}$ & 0.9700 & $\mathrm{C} 35-\mathrm{N} 35$ & 1.455 \\
\hline $\mathrm{C} 21-\mathrm{C} 22$ & $1.389(4)$ & C36-H36 & 0.9300 \\
\hline $\mathrm{C} 21-\mathrm{C} 26$ & $1.391(4)$ & $\mathrm{C} 37-\mathrm{O} 31$ & $1.220(3)$ \\
\hline $\mathrm{C} 22-\mathrm{C} 23$ & $1.393(4)$ & $\mathrm{C} 37-\mathrm{O} 32$ & $1.309(3)$ \\
\hline $\mathrm{C} 22-\mathrm{H} 22$ & 0.9300 & $\mathrm{O} 32-\mathrm{H} 32$ & $1.04(4)$ \\
\hline $\mathrm{C} 23-\mathrm{C} 24$ & $1.371(4)$ & $\mathrm{N} 33-\mathrm{O} 34$ & $1.222(3)$ \\
\hline $\mathrm{C} 23-\mathrm{H} 23$ & 0.9300 & $\mathrm{~N} 33-\mathrm{O} 35$ & $1.223(3)$ \\
\hline $\mathrm{C} 24-\mathrm{C} 25$ & $1.376(4)$ & $\mathrm{N} 35-\mathrm{O} 36$ & $1.218(3)$ \\
\hline $\mathrm{C} 24-\mathrm{O} 24$ & $1.378(3)$ & $\mathrm{N} 35-\mathrm{O} 37$ & $1.229(3)$ \\
\hline $\mathrm{C} 25-\mathrm{C} 26$ & $1.375(4)$ & & \\
\hline $\mathrm{C} 6-\mathrm{N} 1-\mathrm{C} 2$ & $109.4(2)$ & $\mathrm{C} 25-\mathrm{C} 24-\mathrm{O} 24$ & $116.1(3)$ \\
\hline $\mathrm{C} 6-\mathrm{N} 1-\mathrm{H} 11$ & $112.1(18)$ & $\mathrm{C} 26-\mathrm{C} 25-\mathrm{C} 24$ & 121.5 \\
\hline $\mathrm{C} 2-\mathrm{N} 1-\mathrm{H} 11$ & $107.5(18)$ & $\mathrm{C} 26-\mathrm{C} 25-\mathrm{H} 25$ & 119.2 \\
\hline $\mathrm{C} 6-\mathrm{N} 1-\mathrm{H} 12$ & $109.6(18)$ & $\mathrm{C} 24-\mathrm{C} 25-\mathrm{H} 25$ & 119.2 \\
\hline $\mathrm{C} 2-\mathrm{N} 1-\mathrm{H} 12$ & $107.2(18)$ & $\mathrm{C} 25-\mathrm{C} 26-\mathrm{C} 21$ & 121.3 \\
\hline
\end{tabular}




\begin{tabular}{|c|c|c|c|}
\hline $\mathrm{H} 11-\mathrm{N} 1-\mathrm{H} 12$ & $111(3)$ & $\mathrm{C} 25-\mathrm{C} 26-\mathrm{H} 26$ & 119.3 \\
\hline $\mathrm{N} 1-\mathrm{C} 2-\mathrm{C} 3$ & $110.8(2)$ & $\mathrm{C} 21-\mathrm{C} 26-\mathrm{H} 26$ & 119.3 \\
\hline $\mathrm{N} 1-\mathrm{C} 2-\mathrm{H} 2 \mathrm{~A}$ & 109.5 & $\mathrm{C} 24-\mathrm{O} 24-\mathrm{C} 27$ & $117.6(3)$ \\
\hline $\mathrm{C} 3-\mathrm{C} 2-\mathrm{H} 2 \mathrm{~A}$ & 109.5 & $\mathrm{O} 24-\mathrm{C} 27-\mathrm{H} 27 \mathrm{~A}$ & 109.5 \\
\hline $\mathrm{N} 1-\mathrm{C} 2-\mathrm{H} 2 \mathrm{~B}$ & 109.5 & $\mathrm{O} 24-\mathrm{C} 27-\mathrm{H} 27 \mathrm{~B}$ & 109.5 \\
\hline $\mathrm{C} 3-\mathrm{C} 2-\mathrm{H} 2 \mathrm{~B}$ & 109.5 & $\mathrm{H} 27 \mathrm{~A}-\mathrm{C} 27-\mathrm{H} 27 \mathrm{~B}$ & 109.5 \\
\hline $\mathrm{H} 2 \mathrm{~A}-\mathrm{C} 2-\mathrm{H} 2 \mathrm{~B}$ & 108.1 & $\mathrm{O} 24-\mathrm{C} 27-\mathrm{H} 27 \mathrm{C}$ & 109.5 \\
\hline $\mathrm{N} 4-\mathrm{C} 3-\mathrm{C} 2$ & $112.4(2)$ & $\mathrm{H} 27 \mathrm{~A}-\mathrm{C} 27-\mathrm{H} 27 \mathrm{C}$ & 109.5 \\
\hline $\mathrm{N} 4-\mathrm{C} 3-\mathrm{H} 3 \mathrm{~A}$ & 109.1 & $\mathrm{H} 27 \mathrm{~B}-\mathrm{C} 27-\mathrm{H} 27 \mathrm{C}$ & 109.5 \\
\hline $\mathrm{C} 2-\mathrm{C} 3-\mathrm{H} 3 \mathrm{~A}$ & 109.1 & $\mathrm{C} 36-\mathrm{C} 31-\mathrm{C} 32$ & $121.6(2)$ \\
\hline $\mathrm{N} 4-\mathrm{C} 3-\mathrm{H} 3 \mathrm{~B}$ & 109.1 & $\mathrm{C} 36-\mathrm{C} 31-\mathrm{C} 37$ & $118.6(3)$ \\
\hline $\mathrm{C} 2-\mathrm{C} 3-\mathrm{H} 3 \mathrm{~B}$ & 109.1 & $\mathrm{C} 32-\mathrm{C} 31-\mathrm{C} 37$ & $119.8(2)$ \\
\hline $\mathrm{H} 3 \mathrm{~A}-\mathrm{C} 3-\mathrm{H} 3 \mathrm{~B}$ & 107.8 & $\mathrm{O} 33-\mathrm{C} 32-\mathrm{C} 33$ & $124.0(3)$ \\
\hline $\mathrm{C} 21-\mathrm{N} 4-\mathrm{C} 3$ & $114.7(2)$ & $\mathrm{O} 33-\mathrm{C} 32-\mathrm{C} 31$ & $121.0(2)$ \\
\hline $\mathrm{C} 21-\mathrm{N} 4-\mathrm{C} 5$ & $114.4(2)$ & $\mathrm{C} 33-\mathrm{C} 32-\mathrm{C} 31$ & $115.0(2)$ \\
\hline $\mathrm{C} 3-\mathrm{N} 4-\mathrm{C} 5$ & $112.2(2)$ & $\mathrm{C} 34-\mathrm{C} 33-\mathrm{C} 32$ & $123.1(3)$ \\
\hline $\mathrm{N} 4-\mathrm{C} 5-\mathrm{C} 6$ & $111.8(3)$ & $\mathrm{C} 34-\mathrm{C} 33-\mathrm{N} 33$ & $116.7(2)$ \\
\hline $\mathrm{N} 4-\mathrm{C} 5-\mathrm{H} 5 \mathrm{~A}$ & 109.3 & $\mathrm{C} 32-\mathrm{C} 33-\mathrm{N} 33$ & $120.3(2)$ \\
\hline $\mathrm{C} 6-\mathrm{C} 5-\mathrm{H} 5 \mathrm{~A}$ & 109.3 & $\mathrm{C} 33-\mathrm{C} 34-\mathrm{C} 35$ & $119.0(2)$ \\
\hline $\mathrm{N} 4-\mathrm{C} 5-\mathrm{H} 5 \mathrm{~B}$ & 109.3 & $\mathrm{C} 33-\mathrm{C} 34-\mathrm{H} 34$ & 120.5 \\
\hline $\mathrm{C} 6-\mathrm{C} 5-\mathrm{H} 5 \mathrm{~B}$ & 109.3 & $\mathrm{C} 35-\mathrm{C} 34-\mathrm{H} 34$ & 120.5 \\
\hline $\mathrm{H} 5 \mathrm{~A}-\mathrm{C} 5-\mathrm{H} 5 \mathrm{~B}$ & 107.9 & $\mathrm{C} 34-\mathrm{C} 35-\mathrm{C} 36$ & $121.5(2)$ \\
\hline $\mathrm{N} 1-\mathrm{C} 6-\mathrm{C} 5$ & $109.6(2)$ & $\mathrm{C} 34-\mathrm{C} 35-\mathrm{N} 35$ & $119.2(2)$ \\
\hline $\mathrm{N} 1-\mathrm{C} 6-\mathrm{H} 6 \mathrm{~A}$ & 109.7 & $\mathrm{C} 36-\mathrm{C} 35-\mathrm{N} 35$ & $119.4(3)$ \\
\hline $\mathrm{C} 5-\mathrm{C} 6-\mathrm{H} 6 \mathrm{~A}$ & 109.7 & $\mathrm{C} 31-\mathrm{C} 36-\mathrm{C} 35$ & $119.8(3)$ \\
\hline $\mathrm{N} 1-\mathrm{C} 6-\mathrm{H} 6 \mathrm{~B}$ & 109.7 & $\mathrm{C} 31-\mathrm{C} 36-\mathrm{H} 36$ & 120.1 \\
\hline $\mathrm{C} 5-\mathrm{C} 6-\mathrm{H} 6 \mathrm{~B}$ & 109.7 & $\mathrm{C} 35-\mathrm{C} 36-\mathrm{H} 36$ & 120.1 \\
\hline $\mathrm{H} 6 \mathrm{~A}-\mathrm{C} 6-\mathrm{H} 6 \mathrm{~B}$ & 108.2 & $\mathrm{O} 31-\mathrm{C} 37-\mathrm{O} 32$ & $120.1(3)$ \\
\hline $\mathrm{C} 22-\mathrm{C} 21-\mathrm{C} 26$ & $116.6(3)$ & $\mathrm{O} 31-\mathrm{C} 37-\mathrm{C} 31$ & $123.2(3)$ \\
\hline $\mathrm{C} 22-\mathrm{C} 21-\mathrm{N} 4$ & $122.6(3)$ & $\mathrm{O} 32-\mathrm{C} 37-\mathrm{C} 31$ & $116.7(3)$ \\
\hline $\mathrm{C} 26-\mathrm{C} 21-\mathrm{N} 4$ & $120.8(2)$ & $\mathrm{C} 37-\mathrm{O} 32-\mathrm{H} 32$ & $104.6(18)$ \\
\hline $\mathrm{C} 21-\mathrm{C} 22-\mathrm{C} 23$ & $121.8(3)$ & $\mathrm{C} 32-\mathrm{O} 33-\mathrm{H} 32$ & $99.0(13)$ \\
\hline $\mathrm{C} 21-\mathrm{C} 22-\mathrm{H} 22$ & 119.1 & $\mathrm{O} 34-\mathrm{N} 33-\mathrm{O} 35$ & $122.3(3)$ \\
\hline $\mathrm{C} 23-\mathrm{C} 22-\mathrm{H} 22$ & 119.1 & $\mathrm{O} 34-\mathrm{N} 33-\mathrm{C} 33$ & $119.5(3)$ \\
\hline $\mathrm{C} 24-\mathrm{C} 23-\mathrm{C} 22$ & $120.4(3)$ & $\mathrm{O} 35-\mathrm{N} 33-\mathrm{C} 33$ & $118.2(3)$ \\
\hline $\mathrm{C} 24-\mathrm{C} 23-\mathrm{H} 23$ & 119.8 & $\mathrm{O} 36-\mathrm{N} 35-\mathrm{O} 37$ & $122.8(2)$ \\
\hline $\mathrm{C} 22-\mathrm{C} 23-\mathrm{H} 23$ & 119.8 & $\mathrm{O} 36-\mathrm{N} 35-\mathrm{C} 35$ & $118.8(3)$ \\
\hline $\mathrm{C} 23-\mathrm{C} 24-\mathrm{C} 25$ & $118.4(3)$ & $\mathrm{O} 37-\mathrm{N} 35-\mathrm{C} 35$ & $118.5(2)$ \\
\hline $\mathrm{C} 23-\mathrm{C} 24-\mathrm{O} 24$ & $125.6(3)$ & & \\
\hline $\mathrm{C} 6-\mathrm{N} 1-\mathrm{C} 2-\mathrm{C} 3$ & $-58.5(3)$ & $\mathrm{C} 36-\mathrm{C} 31-\mathrm{C} 32-\mathrm{C} 33$ & $2.6(4)$ \\
\hline $\mathrm{N} 1-\mathrm{C} 2-\mathrm{C} 3-\mathrm{N} 4$ & $54.3(3)$ & $\mathrm{C} 37-\mathrm{C} 31-\mathrm{C} 32-\mathrm{C} 33$ & $-176.6(2)$ \\
\hline $\mathrm{C} 2-\mathrm{C} 3-\mathrm{N} 4-\mathrm{C} 21$ & $176.0(2)$ & $\mathrm{O} 33-\mathrm{C} 32-\mathrm{C} 33-\mathrm{C} 34$ & $-179.8(3)$ \\
\hline $\mathrm{C} 2-\mathrm{C} 3-\mathrm{N} 4-\mathrm{C} 5$ & $-51.2(3)$ & $\mathrm{C} 31-\mathrm{C} 32-\mathrm{C} 33-\mathrm{C} 34$ & $-1.3(4)$ \\
\hline $\mathrm{C} 21-\mathrm{N} 4-\mathrm{C} 5-\mathrm{C} 6$ & $-174.0(2)$ & $\mathrm{O} 33-\mathrm{C} 32-\mathrm{C} 33-\mathrm{N} 33$ & $-1.6(4)$ \\
\hline $\mathrm{C} 3-\mathrm{N} 4-\mathrm{C} 5-\mathrm{C} 6$ & $53.0(3)$ & $\mathrm{C} 31-\mathrm{C} 32-\mathrm{C} 33-\mathrm{N} 33$ & $176.9(3)$ \\
\hline $\mathrm{C} 2-\mathrm{N} 1-\mathrm{C} 6-\mathrm{C} 5$ & $60.0(3)$ & $\mathrm{C} 32-\mathrm{C} 33-\mathrm{C} 34-\mathrm{C} 35$ & $-0.9(4)$ \\
\hline $\mathrm{N} 4-\mathrm{C} 5-\mathrm{C} 6-\mathrm{N} 1$ & $-57.5(3)$ & $\mathrm{N} 33-\mathrm{C} 33-\mathrm{C} 34-\mathrm{C} 35$ & $-179.2(2)$ \\
\hline
\end{tabular}




$\begin{array}{ll}\mathrm{C} 3-\mathrm{N} 4-\mathrm{C} 21-\mathrm{C} 22 & -11.2(4) \\ \mathrm{C} 5-\mathrm{N} 4-\mathrm{C} 21-\mathrm{C} 22 & -142.9(3) \\ \mathrm{C} 3-\mathrm{N} 4-\mathrm{C} 21-\mathrm{C} 26 & 170.9(3) \\ \mathrm{C} 5-\mathrm{N} 4-\mathrm{C} 21-\mathrm{C} 26 & 39.2(4) \\ \mathrm{C} 26-\mathrm{C} 21-\mathrm{C} 22-\mathrm{C} 23 & 0.4(5) \\ \mathrm{N} 4-\mathrm{C} 21-\mathrm{C} 22-\mathrm{C} 23 & -177.6(3) \\ \mathrm{C} 21-\mathrm{C} 22-\mathrm{C} 23-\mathrm{C} 24 & -0.8(5) \\ \mathrm{C} 22-\mathrm{C} 23-\mathrm{C} 24-\mathrm{C} 25 & 0.9(5) \\ \mathrm{C} 22-\mathrm{C} 23-\mathrm{C} 24-\mathrm{O} 24 & -179.3(3) \\ \mathrm{C} 23-\mathrm{C} 24-\mathrm{C} 25-\mathrm{C} 26 & -0.6(5) \\ \mathrm{O} 24-\mathrm{C} 24-\mathrm{C} 25-\mathrm{C} 26 & 179.5(3) \\ \mathrm{C} 24-\mathrm{C} 25-\mathrm{C} 26-\mathrm{C} 21 & 0.3(5) \\ \mathrm{C} 22-\mathrm{C} 21-\mathrm{C} 26-\mathrm{C} 25 & -0.2(5) \\ \mathrm{N} 4-\mathrm{C} 21-\mathrm{C} 26-\mathrm{C} 25 & 177.9(3) \\ \mathrm{C} 23-\mathrm{C} 24-\mathrm{O} 24-\mathrm{C} 27 & 0.6(5) \\ \mathrm{C} 25-\mathrm{C} 24-\mathrm{O} 24-\mathrm{C} 27 & -179.6(3) \\ \mathrm{C} 36-\mathrm{C} 31-\mathrm{C} 32-\mathrm{O} 33 & -178.8(3) \\ \mathrm{C} 37-\mathrm{C} 31-\mathrm{C} 32-\mathrm{O} 33 & 2.0(4)\end{array}$

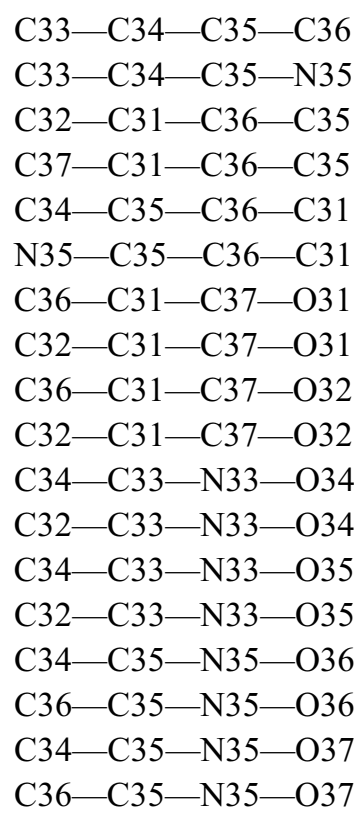

Hydrogen-bond geometry $\left(\AA,{ }^{\circ}\right)$

\begin{tabular}{lllll}
\hline$D-\mathrm{H} \cdots A$ & $D-\mathrm{H}$ & $\mathrm{H} \cdots A$ & $D \cdots A$ & $D-\mathrm{H} \cdots A$ \\
\hline $\mathrm{O} 32-\mathrm{H} 32 \cdots \mathrm{O} 33$ & $1.04(4)$ & $1.47(4)$ & $2.472(3)$ & $158(3)$ \\
$\mathrm{N} 1-\mathrm{H} 11 \cdots \mathrm{O} 33$ & $0.93(3)$ & $1.98(3)$ & $2.820(3)$ & $150(3)$ \\
$\mathrm{N} 1-\mathrm{H} 11 \cdots \mathrm{O} 34$ & $0.93(3)$ & $2.27(3)$ & $2.910(3)$ & $126(2)$ \\
$\mathrm{N} 1-\mathrm{H} 12 \cdots \mathrm{O} 31^{\mathrm{i}}$ & $0.93(3)$ & $2.04(3)$ & $2.931(3)$ & $160(3)$ \\
$\mathrm{N} 1-\mathrm{H} 12 \cdots \mathrm{O} 32^{\mathrm{i}}$ & $0.93(3)$ & $2.58(3)$ & $3.250(3)$ & $129(2)$ \\
$\mathrm{C} 34-\mathrm{H} 34 \cdots \mathrm{O} 36^{\mathrm{ii}}$ & 0.93 & 2.53 & $3.449(3)$ & 171 \\
$\mathrm{C} 5-\mathrm{H} 5 B \cdots C g 2^{\mathrm{iii}}$ & 0.97 & 2.84 & $3.639(3)$ & 140
\end{tabular}

Symmetry codes: (i) $-x+1,-y+1,-z+1$; (ii) $-x+3,-y+2,-z+1$; (iii) $-x+1, y-1 / 2,-z+3 / 2$.

4-(4-Methoxyphenyl)piperazin-1-ium hydrogensuccinate (VIII)

Crystal data

$\mathrm{C}_{11} \mathrm{H}_{17} \mathrm{~N}_{2} \mathrm{O}^{+} \cdot \mathrm{C}_{4} \mathrm{H}_{5} \mathrm{O}_{4}^{-}$

$M_{r}=310.35$

Orthorhombic, Pna2 ${ }_{1}$

$a=9.3225(9) \AA$

$b=28.261(3) \AA$

$c=5.8228(8) \AA$

$V=1534.1(3) \AA^{3}$

$Z=4$

$F(000)=664$

\section{Data collection}

Oxford Diffraction Xcalibur with Sapphire CCD diffractometer

Radiation source: Enhance (Mo) X-ray Source Graphite monochromator $\omega$ scans
$D_{\mathrm{x}}=1.344 \mathrm{Mg} \mathrm{m}^{-3}$

Mo $K \alpha$ radiation, $\lambda=0.71073 \AA$

Cell parameters from 2423 reflections

$\theta=2.6-27.8^{\circ}$

$\mu=0.10 \mathrm{~mm}^{-1}$

$T=296 \mathrm{~K}$

Block, colourless

$0.44 \times 0.42 \times 0.24 \mathrm{~mm}$

Absorption correction: multi-scan

(CrysAlis RED; Oxford Diffraction, 2009)

$T_{\min }=0.816, T_{\max }=0.976$

5828 measured reflections

2419 independent reflections 
2053 reflections with $I>2 \sigma(I)$

$R_{\text {int }}=0.018$

$\theta_{\max }=27.5^{\circ}, \theta_{\min }=2.6^{\circ}$

\section{Refinement}

Refinement on $F^{2}$

Least-squares matrix: full

$R\left[F^{2}>2 \sigma\left(F^{2}\right)\right]=0.043$

$w R\left(F^{2}\right)=0.104$

$S=1.14$

2419 reflections

233 parameters

16 restraints

Primary atom site location: difference Fourier map

Hydrogen site location: mixed

$$
\begin{aligned}
& h=-6 \rightarrow 11 \\
& k=-36 \rightarrow 27 \\
& l=-7 \rightarrow 5
\end{aligned}
$$

$\mathrm{H}$ atoms treated by a mixture of independent and constrained refinement

$$
w=1 /\left[\sigma^{2}\left(F_{\mathrm{o}}^{2}\right)+(0.0459 P)^{2}+0.356 P\right]
$$$$
\text { where } P=\left(F_{\mathrm{o}}^{2}+2 F_{\mathrm{c}}^{2}\right) / 3
$$

$(\Delta / \sigma)_{\max }<0.001$

$\Delta \rho_{\max }=0.16 \mathrm{e} \AA^{-3}$

$\Delta \rho_{\text {min }}=-0.24$ e $\AA^{-3}$

Absolute structure: Flack $x$ determined using 460 quotients $\left[\left(I^{+}\right)-(I)\right] /\left[\left(I^{+}\right)+\left(I^{-}\right)\right]$(Parsons et al., 2013)

\section{Special details}

Experimental. Compound (VIII). IR ( $\left.\mathrm{KBr}, \mathrm{cm}^{-1}\right) 3135\left(\mathrm{NH}_{2}\right), 2836\left(\mathrm{OCH}_{3}\right), 1562(\mathrm{COO})$. NMR $\left.\left(\mathrm{CDCl}_{3}\right) \delta\left({ }^{1} \mathrm{H}\right)\right) 2.66(\mathrm{~s}$, $4 \mathrm{H}$, succinate), $3.32\left(\mathrm{~m}, 4 \mathrm{H}\right.$, piperazine), $3.35\left(\mathrm{~m}, 4 \mathrm{H}\right.$, piperazine), $3.77\left(\mathrm{~s}, 3 \mathrm{H}, \mathrm{OCH}_{3}\right), 6.90(\mathrm{~m}, 4 \mathrm{H}$, methoxyphenyl).

Geometry. All esds (except the esd in the dihedral angle between two 1.s. planes) are estimated using the full covariance matrix. The cell esds are taken into account individually in the estimation of esds in distances, angles and torsion angles;

\begin{tabular}{|c|c|c|c|c|c|}
\hline & $x$ & $y$ & $z$ & $U_{\text {iso }} * / U_{\text {eq }}$ & Occ. $(<1)$ \\
\hline N1 & 0.5210 & $0.66867(8)$ & $0.5428(5)$ & $0.0395(6)$ & \\
\hline H11 & $0.580(3)$ & $0.6921(11)$ & $0.556(7)$ & $0.047^{*}$ & \\
\hline $\mathrm{H} 12$ & $0.435(3)$ & $0.6801(10)$ & $0.623(7)$ & $0.047^{*}$ & \\
\hline $\mathrm{C} 2$ & $0.5853(3)$ & $0.62541(9)$ & $0.6443(7)$ & $0.0435(7)$ & \\
\hline $\mathrm{H} 2 \mathrm{~A}$ & 0.6135 & 0.6317 & 0.8018 & $0.052^{*}$ & \\
\hline $\mathrm{H} 2 \mathrm{~B}$ & 0.6704 & 0.6167 & 0.5586 & $0.052^{*}$ & \\
\hline $\mathrm{C} 3$ & $0.4786(3)$ & $0.58515(9)$ & $0.6388(6)$ & $0.0414(7)$ & \\
\hline $\mathrm{H} 3 \mathrm{~A}$ & 0.5238 & 0.5568 & 0.6992 & $0.050^{*}$ & \\
\hline $\mathrm{H} 3 \mathrm{~B}$ & 0.3981 & 0.5929 & 0.7374 & $0.050^{*}$ & \\
\hline N4 & $0.4259(2)$ & $0.57556(7)$ & $0.4075(5)$ & $0.0347(5)$ & \\
\hline $\mathrm{C} 5$ & $0.3676(3)$ & $0.61875(9)$ & $0.3026(6)$ & $0.0439(7)$ & \\
\hline $\mathrm{H} 5 \mathrm{~A}$ & 0.2824 & 0.6284 & 0.3859 & $0.053^{*}$ & \\
\hline $\mathrm{H} 5 \mathrm{~B}$ & 0.3397 & 0.6120 & 0.1454 & $0.053^{*}$ & \\
\hline C6 & 0.4745 & $0.65878(10)$ & $0.3038(6)$ & $0.0479(8)$ & \\
\hline H6A & 0.5570 & 0.6503 & 0.2110 & $0.058^{*}$ & \\
\hline H6B & 0.4312 & 0.6869 & 0.2380 & $0.058^{*}$ & \\
\hline $\mathrm{C} 21$ & 0.3353 & $0.53502(9)$ & $0.3862(5)$ & $0.0331(6)$ & \\
\hline $\mathrm{C} 22$ & $0.3336(3)$ & $0.49886(9)$ & $0.5496(6)$ & $0.0394(7)$ & \\
\hline $\mathrm{H} 22$ & 0.3926 & 0.5011 & 0.6779 & $0.047^{*}$ & \\
\hline $\mathrm{C} 23$ & $0.2451(3)$ & $0.45967(10)$ & $0.5241(6)$ & $0.0440(7)$ & \\
\hline $\mathrm{H} 23$ & 0.2456 & 0.4361 & 0.6354 & $0.053^{*}$ & \\
\hline $\mathrm{C} 24$ & $0.1567(3)$ & $0.45534(9)$ & $0.3356(6)$ & $0.0407(7)$ & \\
\hline
\end{tabular}
correlations between esds in cell parameters are only used when they are defined by crystal symmetry. An approximate (isotropic) treatment of cell esds is used for estimating esds involving l.s. planes.

Fractional atomic coordinates and isotropic or equivalent isotropic displacement parameters $\left(\AA^{2}\right)$ 


\begin{tabular}{|c|c|c|c|c|c|}
\hline $\mathrm{C} 25$ & 0.1589 & $0.48988(10)$ & $0.1703(7)$ & $0.0484(8)$ & \\
\hline $\mathrm{H} 25$ & 0.1013 & 0.4870 & 0.0406 & $0.058^{*}$ & \\
\hline $\mathrm{C} 26$ & 0.2469 & $0.52924(10)$ & $0.1961(6)$ & $0.0464(8)$ & \\
\hline $\mathrm{H} 26$ & 0.2466 & 0.5524 & 0.0826 & $0.056^{*}$ & \\
\hline $\mathrm{O} 24$ & $0.0728(2)$ & $0.41532(8)$ & $0.3280(5)$ & $0.0620(8)$ & \\
\hline $\mathrm{C} 27$ & -0.0230 & $0.41098(13)$ & $0.1399(9)$ & $0.0729(12)$ & \\
\hline $\mathrm{H} 27 \mathrm{~A}$ & -0.0790 & 0.3827 & 0.1572 & $0.109^{*}$ & \\
\hline H27B & 0.0307 & 0.4094 & -0.0005 & $0.109^{*}$ & \\
\hline $\mathrm{H} 27 \mathrm{C}$ & -0.0856 & 0.4379 & 0.1358 & $0.109^{*}$ & \\
\hline $\mathrm{C} 31$ & $0.7061(15)$ & 0.7714 & $0.797(3)$ & $0.0296(18)$ & $0.660(15)$ \\
\hline $\mathrm{O} 31$ & $0.7173(15)$ & $0.7375(5)$ & 0.655 & $0.0463(18)$ & $0.660(15)$ \\
\hline $\mathrm{O} 32$ & $0.7811(18)$ & $0.8079(5)$ & $0.790(4)$ & $0.0443(15)$ & $0.660(15)$ \\
\hline $\mathrm{C} 32$ & $0.5909(9)$ & $0.7663(3)$ & $0.9790(16)$ & $0.0375(16)$ & $0.660(15)$ \\
\hline $\mathrm{H} 32 \mathrm{~A}$ & 0.6331 & 0.7517 & 1.1138 & $0.045^{*}$ & $0.660(15)$ \\
\hline H32B & 0.5175 & 0.7450 & 0.9216 & $0.045^{*}$ & $0.660(15)$ \\
\hline C33 & 0.5200 & $0.8123(3)$ & 1.0509 (19) & $0.056(3)$ & $0.660(15)$ \\
\hline H33A & 0.5933 & 0.8339 & 1.1050 & $0.067^{*}$ & $0.660(15)$ \\
\hline H33B & 0.4747 & 0.8265 & 0.9178 & $0.067^{*}$ & $0.660(15)$ \\
\hline C34 & $0.4093(16)$ & $0.8060(6)$ & $1.237(3)$ & $0.0468(19)$ & $0.660(15)$ \\
\hline $\mathrm{O} 33$ & $0.3172(11)$ & $0.8361(3)$ & $1.279(2)$ & $0.086(3)$ & $0.660(15)$ \\
\hline O34 & $0.4253(19)$ & $0.7688(5)$ & $1.359(2)$ & $0.065(3)$ & $0.660(15)$ \\
\hline H34 & 0.3533 & 0.7647 & 1.4378 & $0.098^{*}$ & $0.660(15)$ \\
\hline C41 & $0.702(3)$ & $0.7808(8)$ & $0.792(6)$ & $0.0296(18)$ & $0.340(15)$ \\
\hline O41 & $0.736(3)$ & $0.7440(11)$ & $0.678(7)$ & $0.0463(18)$ & $0.340(15)$ \\
\hline $\mathrm{O} 42$ & $0.782(4)$ & $0.8158(10)$ & $0.816(8)$ & $0.0443(15)$ & $0.340(15)$ \\
\hline $\mathrm{C} 42$ & $0.567(2)$ & $0.7768(6)$ & $0.934(3)$ & $0.0375(16)$ & $0.340(15)$ \\
\hline $\mathrm{H} 42 \mathrm{~A}$ & 0.4853 & 0.7819 & 0.8332 & $0.045^{*}$ & $0.340(15)$ \\
\hline H42B & 0.5601 & 0.7447 & 0.9916 & $0.045^{*}$ & $0.340(15)$ \\
\hline $\mathrm{C} 43$ & $0.555(2)$ & $0.8103(7)$ & $1.134(3)$ & $0.056(3)$ & $0.340(15)$ \\
\hline $\mathrm{H} 43 \mathrm{~A}$ & 0.5570 & 0.8425 & 1.0770 & $0.067^{*}$ & $0.340(15)$ \\
\hline H43B & 0.6369 & 0.8060 & 1.2337 & $0.067^{*}$ & $0.340(15)$ \\
\hline $\mathrm{C} 44$ & $0.419(3)$ & $0.8032(11)$ & $1.272(5)$ & $0.0468(19)$ & $0.340(15)$ \\
\hline $\mathrm{O} 43$ & $0.304(2)$ & $0.8221(6)$ & $1.225(5)$ & $0.086(3)$ & $0.340(15)$ \\
\hline $\mathrm{O} 44$ & $0.425(4)$ & $0.7694(12)$ & $1.418(5)$ & $0.065(3)$ & $0.340(15)$ \\
\hline $\mathrm{H} 44$ & 0.3490 & 0.7681 & 1.4892 & $0.098^{*}$ & $0.340(15)$ \\
\hline
\end{tabular}

Atomic displacement parameters $\left(\AA^{2}\right)$

\begin{tabular}{lllllll}
\hline & $U^{11}$ & $U^{22}$ & $U^{33}$ & $U^{12}$ & $U^{13}$ & $U^{23}$ \\
\hline $\mathrm{N} 1$ & $0.0367(12)$ & $0.0305(11)$ & $0.0512(17)$ & $-0.0052(10)$ & $0.0144(13)$ & $-0.0049(11)$ \\
$\mathrm{C} 2$ & $0.0389(14)$ & $0.0366(13)$ & $0.055(2)$ & $-0.0012(11)$ & $-0.0002(16)$ & $-0.0023(14)$ \\
$\mathrm{C} 3$ & $0.0440(15)$ & $0.0361(13)$ & $0.0440(18)$ & $-0.0024(11)$ & $-0.0032(15)$ & $0.0033(13)$ \\
$\mathrm{N} 4$ & $0.0388(12)$ & $0.0296(10)$ & $0.0357(13)$ & $-0.0015(9)$ & $0.0050(11)$ & $-0.0003(10)$ \\
$\mathrm{C} 5$ & $0.0592(17)$ & $0.0331(12)$ & $0.0395(17)$ & $-0.0042(12)$ & $-0.0014(16)$ & $0.0044(13)$ \\
$\mathrm{C} 6$ & $0.0606(18)$ & $0.0386(14)$ & $0.0446(19)$ & $-0.0070(13)$ & $0.0126(18)$ & $0.0056(14)$ \\
$\mathrm{C} 21$ & $0.0345(13)$ & $0.0314(12)$ & $0.0333(16)$ & $0.0023(10)$ & $0.0055(12)$ & $-0.0008(11)$ \\
$\mathrm{C} 22$ & $0.0421(15)$ & $0.0354(13)$ & $0.0406(17)$ & $0.0004(11)$ & $-0.0064(14)$ & $0.0037(13)$ \\
$\mathrm{C} 23$ & $0.0483(16)$ & $0.0366(14)$ & $0.0472(19)$ & $-0.0025(12)$ & $-0.0064(16)$ & $0.0123(13)$
\end{tabular}




\begin{tabular}{lllllll}
$\mathrm{C} 24$ & $0.0370(13)$ & $0.0332(12)$ & $0.052(2)$ & $-0.0019(11)$ & $-0.0022(15)$ & $0.0026(13)$ \\
$\mathrm{C} 25$ & $0.0533(17)$ & $0.0456(15)$ & $0.046(2)$ & $-0.0059(13)$ & $-0.0156(17)$ & $0.0056(15)$ \\
$\mathrm{C} 26$ & $0.0587(18)$ & $0.0414(14)$ & $0.0391(19)$ & $-0.0073(13)$ & $-0.0055(16)$ & $0.0104(14)$ \\
$\mathrm{O} 24$ & $0.0604(13)$ & $0.0480(11)$ & $0.078(2)$ & $-0.0194(10)$ & $-0.0266(15)$ & $0.0143(12)$ \\
$\mathrm{C} 27$ & $0.067(2)$ & $0.061(2)$ & $0.090(3)$ & $-0.0233(17)$ & $-0.036(2)$ & $0.011(2)$ \\
$\mathrm{C} 31$ & $0.0307(14)$ & $0.020(4)$ & $0.0377(18)$ & $0.002(3)$ & $0.0121(15)$ & $0.002(4)$ \\
$\mathrm{O} 31$ & $0.039(4)$ & $0.043(4)$ & $0.056(4)$ & $-0.018(2)$ & $0.025(4)$ & $-0.025(2)$ \\
$\mathrm{O} 32$ & $0.0467(11)$ & $0.029(4)$ & $0.057(5)$ & $-0.011(3)$ & $0.027(2)$ & $-0.014(3)$ \\
$\mathrm{C} 32$ & $0.043(3)$ & $0.025(3)$ & $0.045(4)$ & $0.002(2)$ & $0.019(3)$ & $0.004(2)$ \\
$\mathrm{C} 33$ & $0.069(5)$ & $0.0348(18)$ & $0.064(7)$ & $0.008(3)$ & $0.043(5)$ & $0.009(4)$ \\
$\mathrm{C} 34$ & $0.053(3)$ & $0.032(2)$ & $0.055(5)$ & $0.0033(16)$ & $0.031(3)$ & $-0.001(2)$ \\
$\mathrm{O} 33$ & $0.097(3)$ & $0.054(5)$ & $0.107(7)$ & $0.039(4)$ & $0.067(4)$ & $0.016(4)$ \\
$\mathrm{O} 34$ & $0.0654(15)$ & $0.0655(15)$ & $0.065(8)$ & $0.0228(12)$ & $0.054(5)$ & $0.026(4)$ \\
$\mathrm{C} 41$ & $0.0307(14)$ & $0.020(4)$ & $0.0377(18)$ & $0.002(3)$ & $0.0121(15)$ & $0.002(4)$ \\
$\mathrm{O} 41$ & $0.039(4)$ & $0.043(4)$ & $0.056(4)$ & $-0.018(2)$ & $0.025(4)$ & $-0.025(2)$ \\
$\mathrm{O} 42$ & $0.0467(11)$ & $0.029(4)$ & $0.057(5)$ & $-0.011(3)$ & $0.027(2)$ & $-0.014(3)$ \\
$\mathrm{C} 42$ & $0.043(3)$ & $0.025(3)$ & $0.045(4)$ & $0.002(2)$ & $0.019(3)$ & $0.004(2)$ \\
$\mathrm{C} 43$ & $0.069(5)$ & $0.0348(18)$ & $0.064(7)$ & $0.008(3)$ & $0.043(5)$ & $0.009(4)$ \\
$\mathrm{C} 44$ & $0.053(3)$ & $0.032(2)$ & $0.055(5)$ & $0.0033(16)$ & $0.031(3)$ & $-0.001(2)$ \\
O43 & $0.097(3)$ & $0.054(5)$ & $0.107(7)$ & $0.039(4)$ & $0.067(4)$ & $0.016(4)$ \\
O44 & $0.0654(15)$ & $0.0655(15)$ & $0.065(8)$ & $0.0228(12)$ & $0.054(5)$ & $0.026(4)$ \\
& & & & & & \\
\hline
\end{tabular}

Geometric parameters $\left(\AA,{ }^{\circ}\right)$

\begin{tabular}{llll}
\hline $\mathrm{N} 1-\mathrm{C} 6$ & $1.484(5)$ & $\mathrm{O} 24-\mathrm{C} 27$ & $1.419(5)$ \\
$\mathrm{N} 1-\mathrm{C} 2$ & $1.484(4)$ & $\mathrm{C} 27-\mathrm{H} 27 \mathrm{~A}$ & 0.9600 \\
$\mathrm{~N} 1-\mathrm{H} 11$ & $0.86(3)$ & $\mathrm{C} 27-\mathrm{H} 27 \mathrm{~B}$ & 0.9600 \\
$\mathrm{~N} 1-\mathrm{H} 12$ & $0.98(3)$ & $\mathrm{C} 27-\mathrm{H} 27 \mathrm{C}$ & 0.9600 \\
$\mathrm{C} 2-\mathrm{C} 3$ & $1.512(4)$ & $\mathrm{C} 31-\mathrm{O} 32$ & $1.247(5)$ \\
$\mathrm{C} 2-\mathrm{H} 2 \mathrm{~A}$ & 0.9700 & $\mathrm{C} 31-\mathrm{O} 31$ & $1.270(7)$ \\
$\mathrm{C} 2-\mathrm{H} 2 \mathrm{~B}$ & 0.9700 & $\mathrm{C} 31-\mathrm{C} 32$ & $1.513(6)$ \\
$\mathrm{C} 3-\mathrm{N} 4$ & $1.459(4)$ & $\mathrm{C} 32-\mathrm{C} 33$ & $1.517(7)$ \\
$\mathrm{C} 3-\mathrm{H} 3 \mathrm{~A}$ & 0.9700 & $\mathrm{C} 32-\mathrm{H} 32 \mathrm{~A}$ & 0.9700 \\
$\mathrm{C} 3-\mathrm{H} 3 \mathrm{~B}$ & 0.9700 & $\mathrm{C} 32-\mathrm{H} 32 \mathrm{~B}$ & 0.9700 \\
$\mathrm{~N} 4-\mathrm{C} 21$ & $1.429(3)$ & $\mathrm{C} 33-\mathrm{C} 34$ & $1.505(6)$ \\
$\mathrm{N} 4-\mathrm{C} 5$ & $1.469(3)$ & $\mathrm{C} 33-\mathrm{H} 33 \mathrm{~A}$ & 0.9700 \\
$\mathrm{C} 5-\mathrm{C} 6$ & $1.508(4)$ & $\mathrm{C} 33-\mathrm{H} 33 \mathrm{~B}$ & 0.9700 \\
$\mathrm{C} 5-\mathrm{H} 5 \mathrm{~A}$ & 0.9700 & $\mathrm{C} 34-\mathrm{O} 33$ & $1.233(9)$ \\
$\mathrm{C} 5-\mathrm{H} 5 \mathrm{~B}$ & 0.9700 & $\mathrm{C} 34-\mathrm{O} 34$ & $1.280(6)$ \\
$\mathrm{C} 6-\mathrm{H} 6 \mathrm{~A}$ & 0.9700 & $\mathrm{O} 34-\mathrm{H} 34$ & 0.8200 \\
$\mathrm{C} 6-\mathrm{H} 6 \mathrm{~B}$ & 0.9700 & $\mathrm{C} 41-\mathrm{O} 42$ & $1.247(9)$ \\
$\mathrm{C} 21-\mathrm{C} 26$ & $1.390(4)$ & $\mathrm{C} 41-\mathrm{O} 41$ & $1.271(10)$ \\
$\mathrm{C} 21-\mathrm{C} 22$ & $1.396(4)$ & $\mathrm{C} 41-\mathrm{C} 42$ & $1.514(9)$ \\
$\mathrm{C} 22-\mathrm{C} 23$ & $1.388(4)$ & $\mathrm{C} 42-\mathrm{C} 43$ & $1.507(11)$ \\
$\mathrm{C} 22-\mathrm{H} 22$ & 0.9300 & $\mathrm{C} 42-\mathrm{H} 42 \mathrm{~A}$ & 0.9700 \\
$\mathrm{C} 23-\mathrm{C} 24$ & $1.378(5)$ & $\mathrm{C} 42-\mathrm{H} 42 \mathrm{~B}$ & 0.9700 \\
$\mathrm{C} 23-\mathrm{H} 23$ & 0.9300 & $\mathrm{C} 43-\mathrm{C} 44$ & $1.508(10)$ \\
$\mathrm{C} 24-\mathrm{C} 25$ & $1.371(4)$ & $\mathrm{C} 43-\mathrm{H} 43 \mathrm{~A}$ & 0.9700 \\
& &
\end{tabular}




\begin{tabular}{|c|c|c|c|}
\hline $\mathrm{C} 24-\mathrm{O} 24$ & $1.376(3)$ & $\mathrm{C} 43-\mathrm{H} 43 \mathrm{~B}$ & 0.9700 \\
\hline $\mathrm{C} 25-\mathrm{C} 26$ & $1.390(4)$ & $\mathrm{C} 44-\mathrm{O} 43$ & $1.235(13)$ \\
\hline $\mathrm{C} 25-\mathrm{H} 25$ & 0.9300 & $\mathrm{C} 44-\mathrm{O} 44$ & $1.282(9)$ \\
\hline $\mathrm{C} 26-\mathrm{H} 26$ & 0.9300 & $\mathrm{O} 44-\mathrm{H} 44$ & 0.8200 \\
\hline $\mathrm{C} 6-\mathrm{N} 1-\mathrm{C} 2$ & $109.6(2)$ & $\mathrm{C} 21-\mathrm{C} 26-\mathrm{C} 25$ & $122.0(3)$ \\
\hline $\mathrm{C} 6-\mathrm{N} 1-\mathrm{H} 11$ & $114(3)$ & $\mathrm{C} 21-\mathrm{C} 26-\mathrm{H} 26$ & 119.0 \\
\hline $\mathrm{C} 2-\mathrm{N} 1-\mathrm{H} 11$ & $110(2)$ & $\mathrm{C} 25-\mathrm{C} 26-\mathrm{H} 26$ & 119.0 \\
\hline $\mathrm{C} 6-\mathrm{N} 1-\mathrm{H} 12$ & $106(2)$ & $\mathrm{C} 24-\mathrm{O} 24-\mathrm{C} 27$ & $117.0(3)$ \\
\hline $\mathrm{C} 2-\mathrm{N} 1-\mathrm{H} 12$ & $114(2)$ & $\mathrm{O} 24-\mathrm{C} 27-\mathrm{H} 27 \mathrm{~A}$ & 109.5 \\
\hline $\mathrm{H} 11-\mathrm{N} 1-\mathrm{H} 12$ & $103(3)$ & $\mathrm{O} 24-\mathrm{C} 27-\mathrm{H} 27 \mathrm{~B}$ & 109.5 \\
\hline $\mathrm{N} 1-\mathrm{C} 2-\mathrm{C} 3$ & $110.2(2)$ & $\mathrm{H} 27 \mathrm{~A}-\mathrm{C} 27-\mathrm{H} 27 \mathrm{~B}$ & 109.5 \\
\hline $\mathrm{N} 1-\mathrm{C} 2-\mathrm{H} 2 \mathrm{~A}$ & 109.6 & $\mathrm{O} 24-\mathrm{C} 27-\mathrm{H} 27 \mathrm{C}$ & 109.5 \\
\hline $\mathrm{C} 3-\mathrm{C} 2-\mathrm{H} 2 \mathrm{~A}$ & 109.6 & $\mathrm{H} 27 \mathrm{~A}-\mathrm{C} 27-\mathrm{H} 27 \mathrm{C}$ & 109.5 \\
\hline $\mathrm{N} 1-\mathrm{C} 2-\mathrm{H} 2 \mathrm{~B}$ & 109.6 & $\mathrm{H} 27 \mathrm{~B}-\mathrm{C} 27-\mathrm{H} 27 \mathrm{C}$ & 109.5 \\
\hline $\mathrm{C} 3-\mathrm{C} 2-\mathrm{H} 2 \mathrm{~B}$ & 109.6 & $\mathrm{O} 32-\mathrm{C} 31-\mathrm{O} 31$ & $123.7(8)$ \\
\hline $\mathrm{H} 2 \mathrm{~A}-\mathrm{C} 2-\mathrm{H} 2 \mathrm{~B}$ & 108.1 & $\mathrm{O} 32-\mathrm{C} 31-\mathrm{C} 32$ & $120.0(6)$ \\
\hline $\mathrm{N} 4-\mathrm{C} 3-\mathrm{C} 2$ & $112.4(3)$ & $\mathrm{O} 31-\mathrm{C} 31-\mathrm{C} 32$ & $116.3(6)$ \\
\hline $\mathrm{N} 4-\mathrm{C} 3-\mathrm{H} 3 \mathrm{~A}$ & 109.1 & $\mathrm{C} 31-\mathrm{C} 32-\mathrm{C} 33$ & $114.8(6)$ \\
\hline $\mathrm{C} 2-\mathrm{C} 3-\mathrm{H} 3 \mathrm{~A}$ & 109.1 & $\mathrm{C} 31-\mathrm{C} 32-\mathrm{H} 32 \mathrm{~A}$ & 108.6 \\
\hline $\mathrm{N} 4-\mathrm{C} 3-\mathrm{H} 3 \mathrm{~B}$ & 109.1 & $\mathrm{C} 33-\mathrm{C} 32-\mathrm{H} 32 \mathrm{~A}$ & 108.6 \\
\hline $\mathrm{C} 2-\mathrm{C} 3-\mathrm{H} 3 \mathrm{~B}$ & 109.1 & $\mathrm{C} 31-\mathrm{C} 32-\mathrm{H} 32 \mathrm{~B}$ & 108.6 \\
\hline $\mathrm{H} 3 \mathrm{~A}-\mathrm{C} 3-\mathrm{H} 3 \mathrm{~B}$ & 107.9 & $\mathrm{C} 33-\mathrm{C} 32-\mathrm{H} 32 \mathrm{~B}$ & 108.6 \\
\hline $\mathrm{C} 21-\mathrm{N} 4-\mathrm{C} 3$ & $115.3(2)$ & $\mathrm{H} 32 \mathrm{~A}-\mathrm{C} 32-\mathrm{H} 32 \mathrm{~B}$ & 107.5 \\
\hline $\mathrm{C} 21-\mathrm{N} 4-\mathrm{C} 5$ & $114.3(2)$ & $\mathrm{C} 34-\mathrm{C} 33-\mathrm{C} 32$ & $113.3(6)$ \\
\hline $\mathrm{C} 3-\mathrm{N} 4-\mathrm{C} 5$ & $110.7(2)$ & $\mathrm{C} 34-\mathrm{C} 33-\mathrm{H} 33 \mathrm{~A}$ & 108.9 \\
\hline $\mathrm{N} 4-\mathrm{C} 5-\mathrm{C} 6$ & $112.1(2)$ & $\mathrm{C} 32-\mathrm{C} 33-\mathrm{H} 33 \mathrm{~A}$ & 108.9 \\
\hline $\mathrm{N} 4-\mathrm{C} 5-\mathrm{H} 5 \mathrm{~A}$ & 109.2 & $\mathrm{C} 34-\mathrm{C} 33-\mathrm{H} 33 \mathrm{~B}$ & 108.9 \\
\hline $\mathrm{C} 6-\mathrm{C} 5-\mathrm{H} 5 \mathrm{~A}$ & 109.2 & $\mathrm{C} 32-\mathrm{C} 33-\mathrm{H} 33 \mathrm{~B}$ & 108.9 \\
\hline $\mathrm{N} 4-\mathrm{C} 5-\mathrm{H} 5 \mathrm{~B}$ & 109.2 & $\mathrm{H} 33 \mathrm{~A}-\mathrm{C} 33-\mathrm{H} 33 \mathrm{~B}$ & 107.7 \\
\hline $\mathrm{C} 6-\mathrm{C} 5-\mathrm{H} 5 \mathrm{~B}$ & 109.2 & $\mathrm{O} 33-\mathrm{C} 34-\mathrm{O} 34$ & $122.3(7)$ \\
\hline $\mathrm{H} 5 \mathrm{~A}-\mathrm{C} 5-\mathrm{H} 5 \mathrm{~B}$ & 107.9 & $\mathrm{O} 33-\mathrm{C} 34-\mathrm{C} 33$ & $122.8(7)$ \\
\hline $\mathrm{N} 1-\mathrm{C} 6-\mathrm{C} 5$ & $109.8(3)$ & $\mathrm{O} 34-\mathrm{C} 34-\mathrm{C} 33$ & $114.7(6)$ \\
\hline $\mathrm{N} 1-\mathrm{C} 6-\mathrm{H} 6 \mathrm{~A}$ & 109.7 & $\mathrm{C} 34-\mathrm{O} 34-\mathrm{H} 34$ & 109.5 \\
\hline $\mathrm{C} 5-\mathrm{C} 6-\mathrm{H} 6 \mathrm{~A}$ & 109.7 & $\mathrm{O} 42-\mathrm{C} 41-\mathrm{O} 41$ & $124.3(15)$ \\
\hline $\mathrm{N} 1-\mathrm{C} 6-\mathrm{H} 6 \mathrm{~B}$ & 109.7 & $\mathrm{O} 42-\mathrm{C} 41-\mathrm{C} 42$ & $119.7(12)$ \\
\hline $\mathrm{C} 5-\mathrm{C} 6-\mathrm{H} 6 \mathrm{~B}$ & 109.7 & $\mathrm{O} 41-\mathrm{C} 41-\mathrm{C} 42$ & $115.3(12)$ \\
\hline $\mathrm{H} 6 \mathrm{~A}-\mathrm{C} 6-\mathrm{H} 6 \mathrm{~B}$ & 108.2 & $\mathrm{C} 43-\mathrm{C} 42-\mathrm{C} 41$ & $116.0(12)$ \\
\hline $\mathrm{C} 26-\mathrm{C} 21-\mathrm{C} 22$ & $116.7(2)$ & $\mathrm{C} 43-\mathrm{C} 42-\mathrm{H} 42 \mathrm{~A}$ & 108.3 \\
\hline $\mathrm{C} 26-\mathrm{C} 21-\mathrm{N} 4$ & $120.9(3)$ & $\mathrm{C} 41-\mathrm{C} 42-\mathrm{H} 42 \mathrm{~A}$ & 108.3 \\
\hline $\mathrm{C} 22-\mathrm{C} 21-\mathrm{N} 4$ & $122.3(3)$ & $\mathrm{C} 43-\mathrm{C} 42-\mathrm{H} 42 \mathrm{~B}$ & 108.3 \\
\hline $\mathrm{C} 23-\mathrm{C} 22-\mathrm{C} 21$ & $121.2(3)$ & $\mathrm{C} 41-\mathrm{C} 42-\mathrm{H} 42 \mathrm{~B}$ & 108.3 \\
\hline $\mathrm{C} 23-\mathrm{C} 22-\mathrm{H} 22$ & 119.4 & $\mathrm{H} 42 \mathrm{~A}-\mathrm{C} 42-\mathrm{H} 42 \mathrm{~B}$ & 107.4 \\
\hline $\mathrm{C} 21-\mathrm{C} 22-\mathrm{H} 22$ & 119.4 & $\mathrm{C} 42-\mathrm{C} 43-\mathrm{C} 44$ & $113.1(15)$ \\
\hline $\mathrm{C} 24-\mathrm{C} 23-\mathrm{C} 22$ & 120.8 & $\mathrm{C} 42-\mathrm{C} 43-\mathrm{H} 43 \mathrm{~A}$ & 109.0 \\
\hline $\mathrm{C} 24-\mathrm{C} 23-\mathrm{H} 23$ & 119.6 & $\mathrm{C} 44-\mathrm{C} 43-\mathrm{H} 43 \mathrm{~A}$ & 109.0 \\
\hline $\mathrm{C} 22-\mathrm{C} 23-\mathrm{H} 23$ & 119.6 & $\mathrm{C} 42-\mathrm{C} 43-\mathrm{H} 43 \mathrm{~B}$ & 109.0 \\
\hline $\mathrm{C} 25-\mathrm{C} 24-\mathrm{O} 24$ & $124.8(3)$ & $\mathrm{C} 44-\mathrm{C} 43-\mathrm{H} 43 \mathrm{~B}$ & 109.0 \\
\hline
\end{tabular}




$\begin{array}{ll}\mathrm{C} 25-\mathrm{C} 24-\mathrm{C} 23 & 119.1(3) \\ \mathrm{O} 24-\mathrm{C} 24-\mathrm{C} 23 & 116.0(3) \\ \mathrm{C} 24-\mathrm{C} 25-\mathrm{C} 26 & 120.2(3) \\ \mathrm{C} 24-\mathrm{C} 25-\mathrm{H} 25 & 119.9 \\ \mathrm{C} 26-\mathrm{C} 25-\mathrm{H} 25 & \\ & \\ \mathrm{C} 6-\mathrm{N} 1-\mathrm{C} 2-\mathrm{C} 3 & 56.9(3) \\ \mathrm{N} 1-\mathrm{C} 2-\mathrm{C} 3-\mathrm{N} 4 & 174.2(2) \\ \mathrm{C} 2-\mathrm{C} 3-\mathrm{N} 4-\mathrm{C} 21 & -54.0(3) \\ \mathrm{C} 2-\mathrm{C} 3-\mathrm{N} 4-\mathrm{C} 5 & -172.9(3) \\ \mathrm{C} 21-\mathrm{N} 4-\mathrm{C} 5-\mathrm{C} 6 & 54.8(3) \\ \mathrm{C} 3-\mathrm{N} 4-\mathrm{C} 5-\mathrm{C} 6 & 58.5(3) \\ \mathrm{C} 2-\mathrm{N} 1-\mathrm{C} 6-\mathrm{C} 5 & -57.4(3) \\ \mathrm{N} 4-\mathrm{C} 5-\mathrm{C} 6-\mathrm{N} 1 & 161.9(3) \\ \mathrm{C} 3-\mathrm{N} 4-\mathrm{C} 21-\mathrm{C} 26 & 31.8(4) \\ \mathrm{C} 5-\mathrm{N} 4-\mathrm{C} 21-\mathrm{C} 26 & -19.9(4) \\ \mathrm{C} 3-\mathrm{N} 4-\mathrm{C} 21-\mathrm{C} 22 & -150.0(3) \\ \mathrm{C} 5-\mathrm{N} 4-\mathrm{C} 21-\mathrm{C} 22 & -1.3(4) \\ \mathrm{C} 26-\mathrm{C} 21-\mathrm{C} 22-\mathrm{C} 23 & -179.6(3) \\ \mathrm{N} 4-\mathrm{C} 21-\mathrm{C} 22-\mathrm{C} 23 & 0.2(5) \\ \mathrm{C} 21-\mathrm{C} 22-\mathrm{C} 23-\mathrm{C} 24 & 1.2(5) \\ \mathrm{C} 22-\mathrm{C} 23-\mathrm{C} 24-\mathrm{C} 25 & -179.5(3) \\ \mathrm{C} 22-\mathrm{C} 23-\mathrm{C} 24-\mathrm{O} 24 & \end{array}$

$\begin{array}{ll}\mathrm{H} 43 \mathrm{~A}-\mathrm{C} 43-\mathrm{H} 43 \mathrm{~B} & 107.8 \\ \mathrm{O} 43-\mathrm{C} 44-\mathrm{O} 44 & 120.3(16) \\ \mathrm{O} 43-\mathrm{C} 44-\mathrm{C} 43 & 123.7(14) \\ \mathrm{O} 44-\mathrm{C} 44-\mathrm{C} 43 & 114.8(12) \\ \mathrm{C} 44-\mathrm{O} 44-\mathrm{H} 44 & \\ & \\ \mathrm{O} 24-\mathrm{C} 24-\mathrm{C} 25-\mathrm{C} 26 & 179.3(3) \\ \mathrm{C} 23-\mathrm{C} 24-\mathrm{C} 25-\mathrm{C} 26 & -1.5(5) \\ \mathrm{C} 22-\mathrm{C} 21-\mathrm{C} 26-\mathrm{C} 25 & 1.0(4) \\ \mathrm{N} 4-\mathrm{C} 21-\mathrm{C} 26-\mathrm{C} 25 & 179.3(3) \\ \mathrm{C} 24-\mathrm{C} 25-\mathrm{C} 26-\mathrm{C} 21 & 0.4(5) \\ \mathrm{C} 25-\mathrm{C} 24-\mathrm{O} 24-\mathrm{C} 27 & -3.1(5) \\ \mathrm{C} 23-\mathrm{C} 24-\mathrm{O} 24-\mathrm{C} 27 & 177.7(3) \\ \mathrm{O} 32-\mathrm{C} 31-\mathrm{C} 32-\mathrm{C} 33 & 32(2) \\ \mathrm{O} 31-\mathrm{C} 31-\mathrm{C} 32-\mathrm{C} 33 & -146(2) \\ \mathrm{C} 31-\mathrm{C} 32-\mathrm{C} 33-\mathrm{C} 34 & -178.3(12) \\ \mathrm{C} 32-\mathrm{C} 33-\mathrm{C} 34-\mathrm{O} 33 & -162(2) \\ \mathrm{C} 32-\mathrm{C} 33-\mathrm{C} 34-\mathrm{O} 34 & 23(2) \\ \mathrm{O} 42-\mathrm{C} 41-\mathrm{C} 42-\mathrm{C} 43 & -13(5) \\ \mathrm{O} 41-\mathrm{C} 41-\mathrm{C} 42-\mathrm{C} 43 & 157(4) \\ \mathrm{C} 41-\mathrm{C} 42-\mathrm{C} 43-\mathrm{C} 44 & -178(2) \\ \mathrm{C} 42-\mathrm{C} 43-\mathrm{C} 44-\mathrm{O} 43 & -86(4) \\ \mathrm{C} 42-\mathrm{C} 43-\mathrm{C} 44-\mathrm{O} 44 & 82(4) \\ \end{array}$

Hydrogen-bond geometry $\left(\AA,{ }^{\circ}\right)$

\begin{tabular}{lllll}
\hline$D-\mathrm{H} \cdots A$ & $D-\mathrm{H}$ & $\mathrm{H} \cdots A$ & $D \cdots A$ & $D-\mathrm{H} \cdots A$ \\
\hline $\mathrm{N} 1-\mathrm{H} 11 \cdots \mathrm{O} 31$ & $0.86(3)$ & $1.90(3)$ & $2.750(15)$ & $167(4)$ \\
$\mathrm{N} 1-\mathrm{H} 12 \cdots \mathrm{O} 32^{\mathrm{i}}$ & $0.98(3)$ & $1.77(4)$ & $2.741(19)$ & $171(3)$ \\
$\mathrm{O} 34-\mathrm{H} 34 \cdots \mathrm{O} 31^{\mathrm{ii}}$ & 0.82 & 1.79 & $2.60(2)$ & 168 \\
$\mathrm{~N} 1-\mathrm{H} 11 \cdots \mathrm{O} 41$ & $0.86(3)$ & $2.18(4)$ & $3.03(3)$ & $165(4)$ \\
$\mathrm{N} 1-\mathrm{H} 12 \cdots \mathrm{O} 42^{\mathrm{i}}$ & $0.98(3)$ & $1.82(5)$ & $2.77(4)$ & $163(3)$ \\
$\mathrm{O} 44-\mathrm{H} 44 \cdots \mathrm{O} 41^{\mathrm{ii}}$ & 0.82 & 1.56 & $2.35(2)$ & 161 \\
$\mathrm{C} 3-\mathrm{H} 3 A \cdots \mathrm{Cg} 2^{\mathrm{iii}}$ & 0.97 & 2.76 & $3.652(3)$ & 154 \\
\hline
\end{tabular}

Symmetry codes: (i) $x-1 / 2,-y+3 / 2, z$; (ii) $x-1 / 2,-y+3 / 2, z+1$; (iii) $-x+1,-y+1, z+1 / 2$.

4-(4-Methoxyphenyl)piperazin-1-ium hydrogenfumarate (IX)

Crystal data

$\mathrm{C}_{11} \mathrm{H}_{17} \mathrm{~N}_{2} \mathrm{O}^{+} \cdot \mathrm{C}_{4} \mathrm{H}_{3} \mathrm{O}_{4}^{-}$

$M_{r}=308.33$

Orthorhombic, Pna2 1

$a=9.069(1) \AA$

$b=28.528(3) \AA$

$c=5.8375(9) \AA$

$V=1510.3(3) \AA^{3}$

$Z=4$

$F(000)=656$
$D_{\mathrm{x}}=1.356 \mathrm{Mg} \mathrm{m}^{-3}$

Mo $K \alpha$ radiation, $\lambda=0.71073 \AA$

Cell parameters from 2829 reflections

$\theta=2.7-27.7^{\circ}$

$\mu=0.10 \mathrm{~mm}^{-1}$

$T=296 \mathrm{~K}$

Plate, colourless

$0.48 \times 0.48 \times 0.08 \mathrm{~mm}$ 


\section{Data collection}

Oxford Diffraction Xcalibur with Sapphire CCD diffractometer

Radiation source: Enhance (Mo) X-ray Source Graphite monochromator

$\omega$ scans

Absorption correction: multi-scan

(CrysAlis RED; Oxford Diffraction, 2009)

$T_{\min }=0.888, T_{\max }=0.992$

\section{Refinement}

Refinement on $F^{2}$

Least-squares matrix: full

$R\left[F^{2}>2 \sigma\left(F^{2}\right)\right]=0.041$

$w R\left(F^{2}\right)=0.101$

$S=1.05$

2827 reflections

221 parameters

11 restraints

Primary atom site location: difference Fourier map

Hydrogen site location: mixed
5834 measured reflections

2827 independent reflections

2316 reflections with $I>2 \sigma(I)$

$R_{\text {int }}=0.015$

$\theta_{\max }=27.5^{\circ}, \theta_{\min }=2.7^{\circ}$

$h=-8 \rightarrow 11$

$k=-32 \rightarrow 35$

$l=-7 \rightarrow 6$

$\mathrm{H}$ atoms treated by a mixture of independent and constrained refinement

$w=1 /\left[\sigma^{2}\left(F_{\mathrm{o}}^{2}\right)+(0.0429 P)^{2}+0.4265 P\right]$

where $P=\left(F_{\mathrm{o}}^{2}+2 F_{\mathrm{c}}^{2}\right) / 3$

$(\Delta / \sigma)_{\max }<0.001$

$\Delta \rho_{\max }=0.15$ e $\AA^{-3}$

$\Delta \rho_{\text {min }}=-0.14$ e $\AA^{-3}$

Absolute structure: Flack $x$ determined using 769 quotients $\left[\left(I^{+}\right)-\left(I^{-}\right)\right] /\left[\left(I^{+}\right)+\left(I^{-}\right)\right]$(Parsons et al., 2013)

\section{Special details}

Experimental. Compound (IX). IR ( $\left.\left.\mathrm{KBr}, \mathrm{cm}^{-1}\right) 3001\left(\mathrm{NH}_{2}\right), 2839\left(\mathrm{OCH}_{3}\right), 1562(\mathrm{COO}) . \mathrm{NMR}(\mathrm{CDCl}) \delta\left({ }^{1} \mathrm{H}\right)\right) 3.09(\mathrm{~m}$, $4 \mathrm{H}$, piperazine), $3.35\left(\mathrm{~m}, 4 \mathrm{H}\right.$, piperazine), $3.77\left(\mathrm{~s}, 3 \mathrm{H}, \mathrm{OCH}_{3}\right), 6.26(\mathrm{~s}, 2 \mathrm{H}$, fumarate), 6.90 (m, 4H, methoxyphenyl).

Geometry. All esds (except the esd in the dihedral angle between two 1.s. planes) are estimated using the full covariance matrix. The cell esds are taken into account individually in the estimation of esds in distances, angles and torsion angles; correlations between esds in cell parameters are only used when they are defined by crystal symmetry. An approximate (isotropic) treatment of cell esds is used for estimating esds involving l.s. planes.

Fractional atomic coordinates and isotropic or equivalent isotropic displacement parameters $\left(\AA^{2}\right)$

\begin{tabular}{lllll}
\hline & $x$ & $y$ & $z$ & $U_{\text {iso }} * / U_{\text {eq }}$ \\
N1 & $0.5253(3)$ & $0.66688(8)$ & $0.5154(5)$ & $0.0448(6)$ \\
H11 & $0.588(4)$ & $0.6872(12)$ & $0.518(6)$ & $0.054^{*}$ \\
H12 & $0.440(4)$ & $0.6776(11)$ & $0.596(6)$ & $0.054^{*}$ \\
C2 & $0.5885(3)$ & $0.62425(10)$ & $0.6244(6)$ & $0.0490(8)$ \\
H2A & 0.6182 & 0.6315 & 0.7801 & $0.059^{*}$ \\
H2B & 0.6753 & 0.6144 & 0.5403 & $0.059^{*}$ \\
C3 & $0.4771(3)$ & $0.58500(9)$ & $0.6274(6)$ & $0.0449(7)$ \\
H3A & 0.5225 & 0.5572 & 0.6916 & $0.054^{*}$ \\
H3B & 0.3952 & 0.5937 & 0.7254 & $0.054^{*}$ \\
N4 & $0.4215(3)$ & $0.57432(7)$ & $0.3988(4)$ & $0.0395(6)$ \\
C5 & $0.3637(4)$ & $0.61653(10)$ & $0.2869(6)$ & $0.0505(8)$ \\
H5A & 0.2767 & 0.6272 & 0.3685 & $0.061^{*}$ \\
H5B & 0.3344 & 0.6089 & 0.1315 & $0.061^{*}$ \\
C6 & $0.4751(4)$ & $0.65530(10)$ & $0.2808(6)$ & $0.0540(9)$ \\
H6A & 0.5589 & 0.6458 & 0.1886 & $0.065^{*}$ \\
H6B & 0.4317 & 0.6829 & 0.2106 & $0.065^{*}$ \\
C21 & $0.3300(3)$ & $0.53374(9)$ & $0.3824(5)$ & $0.0372(6)$
\end{tabular}




\begin{tabular}{|c|c|c|c|c|c|}
\hline $\mathrm{C} 22$ & $0.3313(3)$ & $0.49854(9)$ & $0.5482(6)$ & $0.0441(7)$ & \\
\hline $\mathrm{H} 22$ & 0.3919 & 0.5017 & 0.6758 & $0.053^{*}$ & \\
\hline $\mathrm{C} 23$ & $0.2439(3)$ & $0.45907(10)$ & $0.5261(7)$ & $0.0485(8)$ & \\
\hline $\mathrm{H} 23$ & 0.2468 & 0.4361 & 0.6393 & $0.058^{*}$ & \\
\hline $\mathrm{C} 24$ & $0.1529(3)$ & $0.45301(10)$ & $0.3401(6)$ & $0.0456(8)$ & \\
\hline $\mathrm{C} 25$ & $0.1521(4)$ & $0.48719(10)$ & $0.1732(6)$ & $0.0518(8)$ & \\
\hline $\mathrm{H} 25$ & 0.0924 & 0.4836 & 0.0448 & $0.062 *$ & \\
\hline $\mathrm{C} 26$ & $0.2391(4)$ & $0.52673(11)$ & $0.1945(6)$ & $0.0509(8)$ & \\
\hline $\mathrm{H} 26$ & 0.2366 & 0.5493 & 0.0796 & $0.061 *$ & \\
\hline $\mathrm{O} 24$ & $0.0698(3)$ & $0.41291(8)$ & $0.3349(5)$ & $0.0668(8)$ & \\
\hline $\mathrm{C} 27$ & $-0.0259(4)$ & $0.40672(14)$ & $0.1473(9)$ & $0.0804(13)$ & \\
\hline $\mathrm{H} 27 \mathrm{~A}$ & -0.0819 & 0.3785 & 0.1680 & $0.121^{*}$ & \\
\hline $\mathrm{H} 27 \mathrm{~B}$ & 0.0307 & 0.4045 & 0.0088 & $0.121^{*}$ & \\
\hline $\mathrm{H} 27 \mathrm{C}$ & -0.0918 & 0.4330 & 0.1373 & $0.121^{*}$ & \\
\hline $\mathrm{C} 31$ & $0.7171(3)$ & $0.77494(10)$ & $0.8023(6)$ & $0.0452(7)$ & \\
\hline $\mathrm{O} 31$ & $0.7323(3)$ & $0.73835(7)$ & $0.6832(5)$ & $0.0673(8)$ & \\
\hline $\mathrm{O} 32$ & $0.7875(3)$ & $0.81180(7)$ & $0.7776(5)$ & $0.0595(7)$ & \\
\hline $\mathrm{C} 32$ & 0.6095 (4) & $0.77182(10)$ & $0.9941(6)$ & $0.0526(9)$ & $0.906(9)$ \\
\hline H32 & 0.5993 & 0.7427 & 1.0643 & $0.063^{*}$ & $0.906(9)$ \\
\hline $\mathrm{C} 33$ & $0.5305(7)$ & $0.80489(15)$ & $1.0714(12)$ & $0.0607(11)$ & $0.906(9)$ \\
\hline H33 & 0.5456 & 0.8345 & 1.0089 & $0.073^{*}$ & $0.906(9)$ \\
\hline C34 & $0.4163(5)$ & $0.80084(15)$ & $1.2518(8)$ & $0.0518(9)$ & $0.906(9)$ \\
\hline $\mathrm{O} 33$ & $0.3241(5)$ & $0.83097(16)$ & $1.2845(8)$ & $0.0992(18)$ & $0.906(9)$ \\
\hline $\mathrm{O} 34$ & 0.4218 (4) & $0.76290(13)$ & $1.3671(8)$ & $0.0734(13)$ & $0.906(9)$ \\
\hline H34 & 0.3526 & 0.7621 & 1.4574 & $0.110^{*}$ & $0.906(9)$ \\
\hline $\mathrm{C} 42$ & 0.6095 (4) & $0.77182(10)$ & $0.9941(6)$ & $0.0526(9)$ & $0.094(9)$ \\
\hline $\mathrm{H} 42$ & 0.5982 & 0.7421 & 1.0566 & $0.063^{*}$ & $0.094(9)$ \\
\hline $\mathrm{C} 43$ & $0.531(6)$ & $0.8035(9)$ & $1.086(10)$ & $0.0607(11)$ & $0.094(9)$ \\
\hline $\mathrm{H} 43$ & 0.5533 & 0.8343 & 1.0477 & $0.073^{*}$ & $0.094(9)$ \\
\hline C44 & $0.407(4)$ & $0.7961(12)$ & $1.247(6)$ & $0.0518(9)$ & $0.094(9)$ \\
\hline $\mathrm{O} 43$ & $0.280(4)$ & $0.8058(15)$ & $1.203(7)$ & $0.0992(18)$ & $0.094(9)$ \\
\hline O44 & $0.444(4)$ & $0.7801(13)$ & $1.442(6)$ & $0.0734(13)$ & $0.094(9)$ \\
\hline H44 & 0.3697 & 0.7743 & 1.5169 & $0.110^{*}$ & $0.094(9)$ \\
\hline
\end{tabular}

Atomic displacement parameters $\left(\AA^{2}\right)$

\begin{tabular}{lllllll}
\hline & $U^{11}$ & $U^{22}$ & $U^{33}$ & $U^{12}$ & $U^{13}$ & $U^{23}$ \\
\hline $\mathrm{N} 1$ & $0.0436(14)$ & $0.0378(12)$ & $0.0531(18)$ & $-0.0049(10)$ & $0.0167(13)$ & $-0.0020(12)$ \\
$\mathrm{C} 2$ & $0.0444(16)$ & $0.0431(15)$ & $0.060(2)$ & $0.0018(12)$ & $0.0054(16)$ & $-0.0042(15)$ \\
$\mathrm{C} 3$ & $0.0482(17)$ & $0.0398(14)$ & $0.047(2)$ & $-0.0019(13)$ & $-0.0013(15)$ & $0.0052(13)$ \\
$\mathrm{N} 4$ & $0.0466(13)$ & $0.0348(11)$ & $0.0371(14)$ & $0.0016(10)$ & $0.0048(12)$ & $-0.0005(10)$ \\
$\mathrm{C} 5$ & $0.072(2)$ & $0.0403(14)$ & $0.0396(19)$ & $-0.0043(14)$ & $-0.0015(17)$ & $0.0079(14)$ \\
$\mathrm{C} 6$ & $0.077(2)$ & $0.0423(14)$ & $0.043(2)$ & $-0.0044(15)$ & $0.0172(18)$ & $0.0048(15)$ \\
$\mathrm{C} 21$ & $0.0398(15)$ & $0.0355(13)$ & $0.0363(17)$ & $0.0060(11)$ & $0.0060(14)$ & $0.0009(12)$ \\
$\mathrm{C} 22$ & $0.0484(17)$ & $0.0402(14)$ & $0.0437(19)$ & $0.0029(12)$ & $-0.0071(15)$ & $0.0040(14)$ \\
$\mathrm{C} 23$ & $0.0532(18)$ & $0.0413(15)$ & $0.0510(19)$ & $0.0005(13)$ & $-0.0054(17)$ & $0.0123(15)$ \\
$\mathrm{C} 24$ & $0.0391(15)$ & $0.0409(14)$ & $0.057(2)$ & $0.0015(12)$ & $-0.0036(15)$ & $0.0009(14)$ \\
$\mathrm{C} 25$ & $0.0560(19)$ & $0.0519(17)$ & $0.048(2)$ & $-0.0013(15)$ & $-0.0160(17)$ & $0.0035(16)$
\end{tabular}




\begin{tabular}{lllllll}
\hline & & & & & \\
$\mathrm{C} 26$ & $0.065(2)$ & $0.0455(15)$ & $0.042(2)$ & $-0.0041(14)$ & $-0.0061(16)$ & $0.0115(14)$ \\
O24 & $0.0633(15)$ & $0.0532(12)$ & $0.084(2)$ & $-0.0163(11)$ & $-0.0224(15)$ & $0.0125(13)$ \\
C27 & $0.064(2)$ & $0.070(2)$ & $0.107(4)$ & $-0.0205(19)$ & $-0.034(3)$ & $0.012(2)$ \\
C31 & $0.0431(16)$ & $0.0435(15)$ & $0.049(2)$ & $0.0003(12)$ & $0.0227(15)$ & $-0.0016(14)$ \\
O31 & $0.0655(15)$ & $0.0577(13)$ & $0.079(2)$ & $-0.0153(11)$ & $0.0428(14)$ & $-0.0234(13)$ \\
O32 & $0.0632(14)$ & $0.0469(11)$ & $0.0684(17)$ & $-0.0123(10)$ & $0.0369(13)$ & $-0.0050(11)$ \\
C32 & $0.0564(19)$ & $0.0380(14)$ & $0.063(2)$ & $-0.0018(14)$ & $0.0321(18)$ & $-0.0001(15)$ \\
C33 & $0.067(2)$ & $0.0474(16)$ & $0.067(3)$ & $0.0048(16)$ & $0.034(2)$ & $0.0065(17)$ \\
C34 & $0.0558(19)$ & $0.0416(18)$ & $0.058(2)$ & $0.0052(15)$ & $0.0280(18)$ & $-0.0037(15)$ \\
O33 & $0.115(3)$ & $0.064(2)$ & $0.119(3)$ & $0.045(2)$ & $0.081(3)$ & $0.028(2)$ \\
O34 & $0.081(2)$ & $0.0502(19)$ & $0.089(3)$ & $0.0215(17)$ & $0.061(2)$ & $0.0185(17)$ \\
C42 & $0.0564(19)$ & $0.0380(14)$ & $0.063(2)$ & $-0.0018(14)$ & $0.0321(18)$ & $-0.0001(15)$ \\
C43 & $0.067(2)$ & $0.0474(16)$ & $0.067(3)$ & $0.0048(16)$ & $0.034(2)$ & $0.0065(17)$ \\
C44 & $0.0558(19)$ & $0.0416(18)$ & $0.058(2)$ & $0.0052(15)$ & $0.0280(18)$ & $-0.0037(15)$ \\
O43 & $0.115(3)$ & $0.064(2)$ & $0.119(3)$ & $0.045(2)$ & $0.081(3)$ & $0.028(2)$ \\
O44 & $0.081(2)$ & $0.0502(19)$ & $0.089(3)$ & $0.0215(17)$ & $0.061(2)$ & $0.0185(17)$ \\
& & & & & \\
\hline
\end{tabular}

Geometric parameters $\left(\AA,{ }^{\circ}\right)$

\begin{tabular}{|c|c|c|c|}
\hline $\mathrm{N} 1-\mathrm{C} 6$ & $1.481(4)$ & $\mathrm{C} 24-\mathrm{C} 25$ & $1.378(4)$ \\
\hline $\mathrm{N} 1-\mathrm{C} 2$ & $1.487(4)$ & $\mathrm{C} 25-\mathrm{C} 26$ & $1.382(4)$ \\
\hline $\mathrm{N} 1-\mathrm{H} 11$ & $0.81(3)$ & $\mathrm{C} 25-\mathrm{H} 25$ & 0.9300 \\
\hline $\mathrm{N} 1-\mathrm{H} 12$ & $0.96(4)$ & $\mathrm{C} 26-\mathrm{H} 26$ & 0.9300 \\
\hline $\mathrm{C} 2-\mathrm{C} 3$ & $1.508(4)$ & $\mathrm{O} 24-\mathrm{C} 27$ & $1.409(5)$ \\
\hline $\mathrm{C} 2-\mathrm{H} 2 \mathrm{~A}$ & 0.9700 & $\mathrm{C} 27-\mathrm{H} 27 \mathrm{~A}$ & 0.9600 \\
\hline $\mathrm{C} 2-\mathrm{H} 2 \mathrm{~B}$ & 0.9700 & $\mathrm{C} 27-\mathrm{H} 27 \mathrm{~B}$ & 0.9600 \\
\hline $\mathrm{C} 3-\mathrm{N} 4$ & $1.459(4)$ & $\mathrm{C} 27-\mathrm{H} 27 \mathrm{C}$ & 0.9600 \\
\hline $\mathrm{C} 3-\mathrm{H} 3 \mathrm{~A}$ & 0.9700 & $\mathrm{C} 31-\mathrm{O} 32$ & $1.239(3)$ \\
\hline $\mathrm{C} 3-\mathrm{H} 3 \mathrm{~B}$ & 0.9700 & $\mathrm{C} 31-\mathrm{O} 31$ & $1.262(4)$ \\
\hline $\mathrm{N} 4-\mathrm{C} 21$ & $1.428(3)$ & $\mathrm{C} 31-\mathrm{C} 32$ & $1.488(4)$ \\
\hline $\mathrm{N} 4-\mathrm{C} 5$ & $1.467(4)$ & $\mathrm{C} 32-\mathrm{C} 33$ & $1.268(4)$ \\
\hline $\mathrm{C} 5-\mathrm{C} 6$ & $1.499(4)$ & $\mathrm{C} 32-\mathrm{H} 32$ & 0.9300 \\
\hline $\mathrm{C} 5-\mathrm{H} 5 \mathrm{~A}$ & 0.9700 & $\mathrm{C} 33-\mathrm{C} 34$ & $1.481(5)$ \\
\hline $\mathrm{C} 5-\mathrm{H} 5 \mathrm{~B}$ & 0.9700 & $\mathrm{C} 33-\mathrm{H} 33$ & 0.9300 \\
\hline C6-H6A & 0.9700 & $\mathrm{C} 34-\mathrm{O} 33$ & $1.214(4)$ \\
\hline C6-H6B & 0.9700 & $\mathrm{C} 34-\mathrm{O} 34$ & $1.276(5)$ \\
\hline $\mathrm{C} 21-\mathrm{C} 26$ & $1.387(4)$ & O34-H34 & 0.8200 \\
\hline $\mathrm{C} 21-\mathrm{C} 22$ & $1.395(4)$ & $\mathrm{C} 43-\mathrm{C} 44$ & $1.479(12)$ \\
\hline $\mathrm{C} 22-\mathrm{C} 23$ & $1.383(4)$ & $\mathrm{C} 43-\mathrm{H} 43$ & 0.9300 \\
\hline $\mathrm{C} 22-\mathrm{H} 22$ & 0.9300 & $\mathrm{C} 44-\mathrm{O} 43$ & $1.212(12)$ \\
\hline $\mathrm{C} 23-\mathrm{C} 24$ & $1.375(5)$ & $\mathrm{C} 44-\mathrm{O} 44$ & $1.274(12)$ \\
\hline $\mathrm{C} 23-\mathrm{H} 23$ & 0.9300 & $\mathrm{O} 44-\mathrm{H} 44$ & 0.8200 \\
\hline $\mathrm{C} 24-\mathrm{O} 24$ & $1.370(3)$ & & \\
\hline $\mathrm{C} 6-\mathrm{N} 1-\mathrm{C} 2$ & $109.4(2)$ & $\mathrm{C} 21-\mathrm{C} 22-\mathrm{H} 22$ & 119.4 \\
\hline $\mathrm{C} 6-\mathrm{N} 1-\mathrm{H} 11$ & $113(3)$ & $\mathrm{C} 24-\mathrm{C} 23-\mathrm{C} 22$ & $121.3(3)$ \\
\hline $\mathrm{C} 2-\mathrm{N} 1-\mathrm{H} 11$ & $108(2)$ & $\mathrm{C} 24-\mathrm{C} 23-\mathrm{H} 23$ & 119.3 \\
\hline $\mathrm{C} 6-\mathrm{N} 1-\mathrm{H} 12$ & $106(2)$ & $\mathrm{C} 22-\mathrm{C} 23-\mathrm{H} 23$ & 119.3 \\
\hline
\end{tabular}




\begin{tabular}{|c|c|c|c|}
\hline $\mathrm{C} 2-\mathrm{N} 1-\mathrm{H} 12$ & $111(2)$ & $\mathrm{O} 24-\mathrm{C} 24-\mathrm{C} 23$ & $116.9(3)$ \\
\hline $\mathrm{H} 11-\mathrm{N} 1-\mathrm{H} 12$ & $109(3)$ & $\mathrm{O} 24-\mathrm{C} 24-\mathrm{C} 25$ & $124.9(3)$ \\
\hline $\mathrm{N} 1-\mathrm{C} 2-\mathrm{C} 3$ & $110.7(3)$ & $\mathrm{C} 23-\mathrm{C} 24-\mathrm{C} 25$ & $118.2(3)$ \\
\hline $\mathrm{N} 1-\mathrm{C} 2-\mathrm{H} 2 \mathrm{~A}$ & 109.5 & $\mathrm{C} 24-\mathrm{C} 25-\mathrm{C} 26$ & $120.7(3)$ \\
\hline $\mathrm{C} 3-\mathrm{C} 2-\mathrm{H} 2 \mathrm{~A}$ & 109.5 & $\mathrm{C} 24-\mathrm{C} 25-\mathrm{H} 25$ & 119.7 \\
\hline $\mathrm{N} 1-\mathrm{C} 2-\mathrm{H} 2 \mathrm{~B}$ & 109.5 & $\mathrm{C} 26-\mathrm{C} 25-\mathrm{H} 25$ & 119.7 \\
\hline $\mathrm{C} 3-\mathrm{C} 2-\mathrm{H} 2 \mathrm{~B}$ & 109.5 & $\mathrm{C} 25-\mathrm{C} 26-\mathrm{C} 21$ & $121.9(3)$ \\
\hline $\mathrm{H} 2 \mathrm{~A}-\mathrm{C} 2-\mathrm{H} 2 \mathrm{~B}$ & 108.1 & $\mathrm{C} 25-\mathrm{C} 26-\mathrm{H} 26$ & 119.0 \\
\hline $\mathrm{N} 4-\mathrm{C} 3-\mathrm{C} 2$ & $112.1(3)$ & $\mathrm{C} 21-\mathrm{C} 26-\mathrm{H} 26$ & 119.0 \\
\hline $\mathrm{N} 4-\mathrm{C} 3-\mathrm{H} 3 \mathrm{~A}$ & 109.2 & $\mathrm{C} 24-\mathrm{O} 24-\mathrm{C} 27$ & $117.4(3)$ \\
\hline $\mathrm{C} 2-\mathrm{C} 3-\mathrm{H} 3 \mathrm{~A}$ & 109.2 & $\mathrm{O} 24-\mathrm{C} 27-\mathrm{H} 27 \mathrm{~A}$ & 109.5 \\
\hline $\mathrm{N} 4-\mathrm{C} 3-\mathrm{H} 3 \mathrm{~B}$ & 109.2 & $\mathrm{O} 24-\mathrm{C} 27-\mathrm{H} 27 \mathrm{~B}$ & 109.5 \\
\hline $\mathrm{C} 2-\mathrm{C} 3-\mathrm{H} 3 \mathrm{~B}$ & 109.2 & $\mathrm{H} 27 \mathrm{~A}-\mathrm{C} 27-\mathrm{H} 27 \mathrm{~B}$ & 109.5 \\
\hline $\mathrm{H} 3 \mathrm{~A}-\mathrm{C} 3-\mathrm{H} 3 \mathrm{~B}$ & 107.9 & $\mathrm{O} 24-\mathrm{C} 27-\mathrm{H} 27 \mathrm{C}$ & 109.5 \\
\hline $\mathrm{C} 21-\mathrm{N} 4-\mathrm{C} 3$ & $115.6(2)$ & $\mathrm{H} 27 \mathrm{~A}-\mathrm{C} 27-\mathrm{H} 27 \mathrm{C}$ & 109.5 \\
\hline $\mathrm{C} 21-\mathrm{N} 4-\mathrm{C} 5$ & $115.3(2)$ & $\mathrm{H} 27 \mathrm{~B}-\mathrm{C} 27-\mathrm{H} 27 \mathrm{C}$ & 109.5 \\
\hline $\mathrm{C} 3-\mathrm{N} 4-\mathrm{C} 5$ & $111.1(2)$ & $\mathrm{O} 32-\mathrm{C} 31-\mathrm{O} 31$ & $125.6(3)$ \\
\hline $\mathrm{N} 4-\mathrm{C} 5-\mathrm{C} 6$ & $112.0(3)$ & $\mathrm{O} 32-\mathrm{C} 31-\mathrm{C} 32$ & $118.5(3)$ \\
\hline $\mathrm{N} 4-\mathrm{C} 5-\mathrm{H} 5 \mathrm{~A}$ & 109.2 & $\mathrm{O} 31-\mathrm{C} 31-\mathrm{C} 32$ & $115.9(2)$ \\
\hline $\mathrm{C} 6-\mathrm{C} 5-\mathrm{H} 5 \mathrm{~A}$ & 109.2 & $\mathrm{C} 33-\mathrm{C} 32-\mathrm{C} 31$ & $126.4(3)$ \\
\hline $\mathrm{N} 4-\mathrm{C} 5-\mathrm{H} 5 \mathrm{~B}$ & 109.2 & $\mathrm{C} 33-\mathrm{C} 32-\mathrm{H} 32$ & 116.8 \\
\hline $\mathrm{C} 6-\mathrm{C} 5-\mathrm{H} 5 \mathrm{~B}$ & 109.2 & $\mathrm{C} 31-\mathrm{C} 32-\mathrm{H} 32$ & 116.8 \\
\hline $\mathrm{H} 5 \mathrm{~A}-\mathrm{C} 5-\mathrm{H} 5 \mathrm{~B}$ & 107.9 & $\mathrm{C} 32-\mathrm{C} 33-\mathrm{C} 34$ & $126.2(4)$ \\
\hline $\mathrm{N} 1-\mathrm{C} 6-\mathrm{C} 5$ & $110.5(3)$ & $\mathrm{C} 32-\mathrm{C} 33-\mathrm{H} 33$ & 116.9 \\
\hline $\mathrm{N} 1-\mathrm{C} 6-\mathrm{H} 6 \mathrm{~A}$ & 109.6 & $\mathrm{C} 34-\mathrm{C} 33-\mathrm{H} 33$ & 116.9 \\
\hline $\mathrm{C} 5-\mathrm{C} 6-\mathrm{H} 6 \mathrm{~A}$ & 109.6 & $\mathrm{O} 33-\mathrm{C} 34-\mathrm{O} 34$ & $123.0(4)$ \\
\hline $\mathrm{N} 1-\mathrm{C} 6-\mathrm{H} 6 \mathrm{~B}$ & 109.6 & $\mathrm{O} 33-\mathrm{C} 34-\mathrm{C} 33$ & $122.5(4)$ \\
\hline $\mathrm{C} 5-\mathrm{C} 6-\mathrm{H} 6 \mathrm{~B}$ & 109.6 & $\mathrm{O} 34-\mathrm{C} 34-\mathrm{C} 33$ & $114.5(3)$ \\
\hline $\mathrm{H} 6 \mathrm{~A}-\mathrm{C} 6-\mathrm{H} 6 \mathrm{~B}$ & 108.1 & $\mathrm{C} 34-\mathrm{O} 34-\mathrm{H} 34$ & 109.5 \\
\hline $\mathrm{C} 26-\mathrm{C} 21-\mathrm{C} 22$ & $116.7(3)$ & $\mathrm{C} 44-\mathrm{C} 43-\mathrm{H} 43$ & 116.8 \\
\hline $\mathrm{C} 26-\mathrm{C} 21-\mathrm{N} 4$ & $121.0(3)$ & $\mathrm{O} 43-\mathrm{C} 44-\mathrm{O} 44$ & $121.3(18)$ \\
\hline $\mathrm{C} 22-\mathrm{C} 21-\mathrm{N} 4$ & $122.2(3)$ & $\mathrm{O} 43-\mathrm{C} 44-\mathrm{C} 43$ & $124(2)$ \\
\hline $\mathrm{C} 23-\mathrm{C} 22-\mathrm{C} 21$ & $121.1(3)$ & $\mathrm{O} 44-\mathrm{C} 44-\mathrm{C} 43$ & $114.9(18)$ \\
\hline $\mathrm{C} 23-\mathrm{C} 22-\mathrm{H} 22$ & 119.4 & $\mathrm{C} 44-\mathrm{O} 44-\mathrm{H} 44$ & 109.5 \\
\hline $\mathrm{C} 6-\mathrm{N} 1-\mathrm{C} 2-\mathrm{C} 3$ & $-57.2(3)$ & $\mathrm{C} 22-\mathrm{C} 23-\mathrm{C} 24-\mathrm{O} 24$ & $-179.4(3)$ \\
\hline $\mathrm{N} 1-\mathrm{C} 2-\mathrm{C} 3-\mathrm{N} 4$ & $55.8(3)$ & $\mathrm{C} 22-\mathrm{C} 23-\mathrm{C} 24-\mathrm{C} 25$ & $0.8(5)$ \\
\hline $\mathrm{C} 2-\mathrm{C} 3-\mathrm{N} 4-\mathrm{C} 21$ & $172.3(2)$ & $\mathrm{O} 24-\mathrm{C} 24-\mathrm{C} 25-\mathrm{C} 26$ & $179.4(3)$ \\
\hline $\mathrm{C} 2-\mathrm{C} 3-\mathrm{N} 4-\mathrm{C} 5$ & $-53.8(3)$ & $\mathrm{C} 23-\mathrm{C} 24-\mathrm{C} 25-\mathrm{C} 26$ & $-0.9(5)$ \\
\hline $\mathrm{C} 21-\mathrm{N} 4-\mathrm{C} 5-\mathrm{C} 6$ & $-171.4(3)$ & $\mathrm{C} 24-\mathrm{C} 25-\mathrm{C} 26-\mathrm{C} 21$ & $0.0(5)$ \\
\hline $\mathrm{C} 3-\mathrm{N} 4-\mathrm{C} 5-\mathrm{C} 6$ & $54.7(3)$ & $\mathrm{C} 22-\mathrm{C} 21-\mathrm{C} 26-\mathrm{C} 25$ & $0.9(5)$ \\
\hline $\mathrm{C} 2-\mathrm{N} 1-\mathrm{C} 6-\mathrm{C} 5$ & $57.9(3)$ & $\mathrm{N} 4-\mathrm{C} 21-\mathrm{C} 26-\mathrm{C} 25$ & $178.6(3)$ \\
\hline $\mathrm{N} 4-\mathrm{C} 5-\mathrm{C} 6-\mathrm{N} 1$ & $-57.2(4)$ & $\mathrm{C} 23-\mathrm{C} 24-\mathrm{O} 24-\mathrm{C} 27$ & $178.8(3)$ \\
\hline $\mathrm{C} 3-\mathrm{N} 4-\mathrm{C} 21-\mathrm{C} 26$ & $162.4(3)$ & $\mathrm{C} 25-\mathrm{C} 24-\mathrm{O} 24-\mathrm{C} 27$ & $-1.4(5)$ \\
\hline $\mathrm{C} 5-\mathrm{N} 4-\mathrm{C} 21-\mathrm{C} 26$ & $30.5(4)$ & $\mathrm{O} 32-\mathrm{C} 31-\mathrm{C} 32-\mathrm{C} 33$ & $34.7(7)$ \\
\hline $\mathrm{C} 3-\mathrm{N} 4-\mathrm{C} 21-\mathrm{C} 22$ & $-20.1(4)$ & $\mathrm{O} 31-\mathrm{C} 31-\mathrm{C} 32-\mathrm{C} 33$ & $-148.0(6)$ \\
\hline $\mathrm{C} 5-\mathrm{N} 4-\mathrm{C} 21-\mathrm{C} 22$ & $-151.9(3)$ & $\mathrm{C} 31-\mathrm{C} 32-\mathrm{C} 33-\mathrm{C} 34$ & $175.5(5)$ \\
\hline $\mathrm{C} 26-\mathrm{C} 21-\mathrm{C} 22-\mathrm{C} 23$ & $-0.9(4)$ & $\mathrm{C} 32-\mathrm{C} 33-\mathrm{C} 34-\mathrm{O} 33$ & $-164.1(8)$ \\
\hline
\end{tabular}


$\mathrm{N} 4-\mathrm{C} 21-\mathrm{C} 22-\mathrm{C} 23$

$-178.6(3)$

$\mathrm{C} 32-\mathrm{C} 33-\mathrm{C} 34-\mathrm{O} 34$

$14.9(10)$

$\mathrm{C} 21-\mathrm{C} 22-\mathrm{C} 23-\mathrm{C} 24$

$0.1(5)$

Hydrogen-bond geometry $\left(\AA,^{\circ}\right)$

\begin{tabular}{lllll}
\hline$D-\mathrm{H} \cdots A$ & $D-\mathrm{H}$ & $\mathrm{H} \cdots A$ & $D \cdots A$ & $D-\mathrm{H} \cdots A$ \\
\hline $\mathrm{N} 1-\mathrm{H} 11 \cdots \mathrm{O} 31$ & $0.81(4)$ & $2.18(3)$ & $2.940(4)$ & $155(3)$ \\
$\mathrm{N} 1-\mathrm{H} 12 \cdots \mathrm{O} 32^{\mathrm{i}}$ & $0.96(4)$ & $1.77(4)$ & $2.714(4)$ & $169(3)$ \\
$\mathrm{O} 34-\mathrm{H} 34 \cdots \mathrm{O} 31^{\mathrm{ii}}$ & 0.82 & 1.71 & $2.522(5)$ & 170 \\
$\mathrm{O} 44-\mathrm{H} 44 \cdots \mathrm{O} 31^{\mathrm{ii}}$ & 0.82 & 1.62 & $2.44(2)$ & 175 \\
$\mathrm{C} 3-\mathrm{H} 3 A \cdots \mathrm{Cg} 2^{\mathrm{iii}}$ & 0.97 & 2.76 & $3.650(3)$ & 153 \\
\hline
\end{tabular}

Symmetry codes: (i) $x-1 / 2,-y+3 / 2, z$; (ii) $x-1 / 2,-y+3 / 2, z+1$; (iii) $-x+1,-y+1, z+1 / 2$.

4-(4-Methoxyphenyl)piperazin-1-ium hydrogenmaleate (X)

\section{Crystal data}

\section{$\mathrm{C}_{11} \mathrm{H}_{17} \mathrm{~N}_{2} \mathrm{O}^{+} \cdot \mathrm{C}_{4} \mathrm{H}_{3} \mathrm{O}_{4}^{-}$}

$M_{r}=308.33$

Monoclinic, $P 2_{1} / c$

$a=9.063$ (1) $\AA$

$b=6.4956(9) \AA$

$c=26.093(3) \AA$

$\beta=93.18(1)^{\circ}$

$V=1533.7(3) \AA^{3}$

$Z=4$

\section{Data collection}

Oxford Diffraction Xcalibur with Sapphire CCD diffractometer

Radiation source: Enhance (Mo) X-ray Source

Graphite monochromator

$\omega$ scans

Absorption correction: multi-scan

(CrysAlis RED; Oxford Diffraction, 2009)

$T_{\min }=0.871, T_{\max }=0.968$

\section{Refinement}

Refinement on $F^{2}$

Least-squares matrix: full

$R\left[F^{2}>2 \sigma\left(F^{2}\right)\right]=0.040$

$w R\left(F^{2}\right)=0.111$

$S=1.05$

3311 reflections

210 parameters

0 restraints

Primary atom site location: difference Fourier map

Hydrogen site location: mixed
$F(000)=656$

$D_{\mathrm{x}}=1.335 \mathrm{Mg} \mathrm{m}^{-3}$

Mo $K \alpha$ radiation, $\lambda=0.71073 \AA$

Cell parameters from 3315 reflections

$\theta=2.7-27.8^{\circ}$

$\mu=0.10 \mathrm{~mm}^{-1}$

$T=296 \mathrm{~K}$

Block, colourless

$0.48 \times 0.44 \times 0.32 \mathrm{~mm}$

6112 measured reflections

3311 independent reflections

2459 reflections with $I>2 \sigma(I)$

$R_{\text {int }}=0.014$

$\theta_{\max }=27.6^{\circ}, \theta_{\min }=2.7^{\circ}$

$h=-11 \rightarrow 11$

$k=-6 \rightarrow 8$

$l=-26 \rightarrow 33$

$\mathrm{H}$ atoms treated by a mixture of independent and constrained refinement

$w=1 /\left[\sigma^{2}\left(F_{\mathrm{o}}^{2}\right)+(0.0521 P)^{2}+0.3046 P\right]$ where $P=\left(F_{\mathrm{o}}^{2}+2 F_{\mathrm{c}}^{2}\right) / 3$

$(\Delta / \sigma)_{\max }=0.001$

$\Delta \rho_{\max }=0.21 \mathrm{e} \AA^{-3}$

$\Delta \rho_{\min }=-0.13$ e $\AA^{-3}$

Extinction correction: SHELXL, $\mathrm{Fc}^{*}=\mathrm{kFc}\left[1+0.001 \mathrm{xFc}^{2} \lambda^{3} / \sin (2 \theta)\right]^{-1 / 4}$

Extinction coefficient: 0.0192 (18) 


\section{Special details}

Experimental. Compound (X). IR ( $\left.\mathrm{KBr}, \mathrm{cm}^{-1}\right) 3073\left(\mathrm{NH}_{2}\right), 2836\left(\mathrm{OCH}_{3}\right), 1565(\mathrm{COO})$. NMR $\left.\left(\mathrm{CDCl}_{3}\right) \delta\left({ }^{1} \mathrm{H}\right)\right) 3.34(\mathrm{~m}$, $4 \mathrm{H}$, piperazine), $3.41\left(\mathrm{~m}, 4 \mathrm{H}\right.$, piperazine), $3.77\left(\mathrm{~s}, 3 \mathrm{H}, \mathrm{OCH}_{3}\right), 6.29(\mathrm{~s}, 2 \mathrm{H}$, maleate), $6.90(\mathrm{~m}, 4 \mathrm{H}$, methoxyphenyl).

Geometry. All esds (except the esd in the dihedral angle between two 1.s. planes) are estimated using the full covariance matrix. The cell esds are taken into account individually in the estimation of esds in distances, angles and torsion angles; correlations between esds in cell parameters are only used when they are defined by crystal symmetry. An approximate (isotropic) treatment of cell esds is used for estimating esds involving l.s. planes.

Fractional atomic coordinates and isotropic or equivalent isotropic displacement parameters $\left(\AA^{2}\right)$

\begin{tabular}{|c|c|c|c|c|}
\hline & $x$ & $y$ & $z$ & $U_{\text {iso }} * / U_{\text {eq }}$ \\
\hline N1 & $0.54059(15)$ & $0.1806(2)$ & $0.56144(5)$ & $0.0431(3)$ \\
\hline H11 & $0.6147(19)$ & $0.166(3)$ & $0.5393(6)$ & $0.052 *$ \\
\hline $\mathrm{H} 12$ & $0.452(2)$ & $0.174(3)$ & $0.5426(7)$ & $0.052 *$ \\
\hline $\mathrm{C} 2$ & $0.5537(2)$ & $0.3928(3)$ & $0.58165(6)$ & $0.0531(4)$ \\
\hline $\mathrm{H} 2 \mathrm{~A}$ & 0.5428 & 0.4903 & 0.5535 & $0.064^{*}$ \\
\hline $\mathrm{H} 2 \mathrm{~B}$ & 0.6506 & 0.4121 & 0.5986 & $0.064^{*}$ \\
\hline $\mathrm{C} 3$ & $0.43600(18)$ & $0.4321(2)$ & $0.61930(6)$ & $0.0468(4)$ \\
\hline $\mathrm{H} 3 \mathrm{~A}$ & 0.4472 & 0.5706 & 0.6329 & $0.056^{*}$ \\
\hline H3B & 0.3392 & 0.4222 & 0.6016 & $0.056^{*}$ \\
\hline N4 & $0.44597(13)$ & $0.28484(17)$ & $0.66144(4)$ & $0.0362(3)$ \\
\hline $\mathrm{C} 5$ & $0.43395(19)$ & $0.0748(2)$ & $0.64123(6)$ & $0.0481(4)$ \\
\hline $\mathrm{H} 5 \mathrm{~A}$ & 0.3370 & 0.0559 & 0.6243 & $0.058^{*}$ \\
\hline $\mathrm{H} 5 \mathrm{~B}$ & 0.4438 & -0.0222 & 0.6695 & $0.058^{*}$ \\
\hline C6 & $0.55084(18)$ & $0.0302(2)$ & $0.60367(6)$ & $0.0468(4)$ \\
\hline H6A & 0.6480 & 0.0377 & 0.6211 & $0.056^{*}$ \\
\hline H6B & 0.5373 & -0.1077 & 0.5900 & $0.056^{*}$ \\
\hline $\mathrm{C} 21$ & $0.35362(15)$ & $0.3268(2)$ & $0.70263(5)$ & $0.0354(3)$ \\
\hline $\mathrm{C} 22$ & $0.26577(18)$ & $0.5006(2)$ & $0.70426(6)$ & $0.0481(4)$ \\
\hline $\mathrm{H} 22$ & 0.2632 & 0.5920 & 0.6768 & $0.058^{*}$ \\
\hline $\mathrm{C} 23$ & $0.18120(18)$ & $0.5421(3)$ & $0.74593(6)$ & $0.0530(4)$ \\
\hline $\mathrm{H} 23$ & 0.1247 & 0.6616 & 0.7463 & $0.064^{*}$ \\
\hline $\mathrm{C} 24$ & $0.18071(17)$ & $0.4076(3)$ & $0.78649(5)$ & $0.0489(4)$ \\
\hline $\mathrm{C} 25$ & $0.2673(2)$ & $0.2332(3)$ & $0.78540(6)$ & $0.0537(4)$ \\
\hline $\mathrm{H} 25$ & 0.2679 & 0.1410 & 0.8127 & $0.064^{*}$ \\
\hline $\mathrm{C} 26$ & $0.35276(18)$ & $0.1936(3)$ & $0.74451(6)$ & $0.0464(4)$ \\
\hline H326 & 0.4110 & 0.0757 & 0.7448 & $0.056^{*}$ \\
\hline $\mathrm{O} 24$ & $0.10103(14)$ & $0.4323(2)$ & $0.82944(4)$ & $0.0706(4)$ \\
\hline $\mathrm{C} 27$ & $0.0207(2)$ & $0.6185(4)$ & $0.83401(7)$ & $0.0844(7)$ \\
\hline $\mathrm{H} 27 \mathrm{~A}$ & -0.0231 & 0.6219 & 0.8667 & $0.127^{*}$ \\
\hline H27B & -0.0556 & 0.6260 & 0.8070 & $0.127^{*}$ \\
\hline $\mathrm{H} 27 \mathrm{C}$ & 0.0864 & 0.7334 & 0.8314 & $0.127^{*}$ \\
\hline $\mathrm{C} 31$ & $0.81864(16)$ & $0.2456(2)$ & $0.48041(7)$ & $0.0439(4)$ \\
\hline $\mathrm{C} 32$ & $0.93279(17)$ & $0.2644(2)$ & $0.44164(6)$ & $0.0460(4)$ \\
\hline H32 & 0.8951 & 0.2815 & 0.4080 & $0.055^{*}$ \\
\hline $\mathrm{C} 33$ & $1.07926(16)$ & $0.2605(2)$ & $0.44719(6)$ & $0.0429(4)$ \\
\hline $\mathrm{H} 33$ & 1.1274 & 0.2743 & 0.4168 & $0.052 *$ \\
\hline $\mathrm{C} 34$ & $1.17846(15)$ & $0.2380(2)$ & $0.49393(6)$ & $0.0370(3)$ \\
\hline
\end{tabular}




$\begin{array}{lllll}\text { O31 } & 0.68826(12) & 0.23449(19) & 0.46522(5) & 0.0627(4) \\ \text { O32 } & 0.85878(12) & 0.23939(18) & 0.52832(4) & 0.0520(3) \\ \text { O33 } & 1.12495(12) & 0.23595(18) & 0.53839(4) & 0.0488(3) \\ \text { H33A } & 0.996(2) & 0.240(3) & 0.5354(8) & 0.073^{*} \\ \text { O34 } & 1.31216(11) & 0.22095(17) & 0.48789(4) & 0.0495(3)\end{array}$

Atomic displacement parameters $\left(\AA^{2}\right)$

\begin{tabular}{lllllll}
\hline & $U^{11}$ & $U^{22}$ & $U^{33}$ & $U^{12}$ & $U^{13}$ & $U^{23}$ \\
\hline $\mathrm{N} 1$ & $0.0357(6)$ & $0.0568(8)$ & $0.0375(7)$ & $0.0007(6)$ & $0.0084(5)$ & $-0.0023(6)$ \\
$\mathrm{C} 2$ & $0.0645(10)$ & $0.0474(9)$ & $0.0493(9)$ & $-0.0055(8)$ & $0.0214(8)$ & $0.0037(7)$ \\
$\mathrm{C} 3$ & $0.0590(10)$ & $0.0388(8)$ & $0.0440(8)$ & $0.0018(7)$ & $0.0146(7)$ & $0.0034(7)$ \\
$\mathrm{N} 4$ & $0.0396(6)$ & $0.0349(6)$ & $0.0346(6)$ & $0.0002(5)$ & $0.0054(5)$ & $0.0009(5)$ \\
$\mathrm{C} 5$ & $0.0591(10)$ & $0.0385(8)$ & $0.0484(9)$ & $-0.0012(7)$ & $0.0186(7)$ & $0.0000(7)$ \\
$\mathrm{C} 6$ & $0.0507(9)$ & $0.0450(9)$ & $0.0454(8)$ & $0.0080(7)$ & $0.0087(7)$ & $-0.0013(7)$ \\
C21 & $0.0340(7)$ & $0.0391(7)$ & $0.0329(7)$ & $-0.0023(6)$ & $0.0007(5)$ & $-0.0016(6)$ \\
C22 & $0.0550(9)$ & $0.0482(9)$ & $0.0419(8)$ & $0.0101(7)$ & $0.0106(7)$ & $0.0080(7)$ \\
C23 & $0.0539(10)$ & $0.0584(10)$ & $0.0472(9)$ & $0.0176(8)$ & $0.0085(7)$ & $0.0009(8)$ \\
C24 & $0.0435(8)$ & $0.0715(11)$ & $0.0316(7)$ & $0.0060(8)$ & $0.0023(6)$ & $-0.0037(7)$ \\
C25 & $0.0645(10)$ & $0.0662(11)$ & $0.0305(7)$ & $0.0109(9)$ & $0.0042(7)$ & $0.0111(7)$ \\
C26 & $0.0525(9)$ & $0.0508(9)$ & $0.0358(8)$ & $0.0120(7)$ & $0.0001(6)$ & $0.0044(6)$ \\
O24 & $0.0722(8)$ & $0.1024(11)$ & $0.0386(6)$ & $0.0251(7)$ & $0.0175(6)$ & $0.0033(6)$ \\
C27 & $0.0759(13)$ & $0.126(2)$ & $0.0528(11)$ & $0.0418(14)$ & $0.0177(10)$ & $-0.0042(12)$ \\
C31 & $0.0357(8)$ & $0.0337(8)$ & $0.0631(10)$ & $0.0035(6)$ & $0.0104(7)$ & $0.0045(7)$ \\
C32 & $0.0425(8)$ & $0.0529(9)$ & $0.0427(8)$ & $0.0026(7)$ & $0.0035(6)$ & $0.0069(7)$ \\
C33 & $0.0411(8)$ & $0.0493(9)$ & $0.0395(8)$ & $0.0003(7)$ & $0.0118(6)$ & $0.0033(7)$ \\
C34 & $0.0352(7)$ & $0.0300(7)$ & $0.0463(8)$ & $-0.0020(6)$ & $0.0071(6)$ & $-0.0005(6)$ \\
O31 & $0.0334(6)$ & $0.0690(8)$ & $0.0858(9)$ & $0.0036(5)$ & $0.0040(6)$ & $0.0110(7)$ \\
O32 & $0.0418(6)$ & $0.0641(8)$ & $0.0517(7)$ & $0.0009(5)$ & $0.0172(5)$ & $0.0003(5)$ \\
O33 & $0.0427(6)$ & $0.0647(8)$ & $0.0393(6)$ & $0.0007(5)$ & $0.0056(4)$ & $-0.0020(5)$ \\
O34 & $0.0337(5)$ & $0.0572(7)$ & $0.0580(7)$ & $-0.0010(5)$ & $0.0069(5)$ & $0.0012(5)$ \\
& & & & & & \\
& & & & & & \\
& & & & & & \\
& & & & & & \\
& & & & &
\end{tabular}

Geometric parameters $\left(\AA,{ }^{\circ}\right)$

\begin{tabular}{llll}
\hline $\mathrm{N} 1-\mathrm{C} 6$ & $1.472(2)$ & $\mathrm{C} 23-\mathrm{H} 23$ & 0.9300 \\
$\mathrm{~N} 1-\mathrm{C} 2$ & $1.478(2)$ & $\mathrm{C} 24-\mathrm{O} 24$ & $1.3759(17)$ \\
$\mathrm{N} 1-\mathrm{H} 11$ & $0.915(17)$ & $\mathrm{C} 24-\mathrm{C} 25$ & $1.379(2)$ \\
$\mathrm{N} 1-\mathrm{H} 12$ & $0.917(18)$ & $\mathrm{C} 25-\mathrm{C} 26$ & $1.377(2)$ \\
$\mathrm{C} 2-\mathrm{C} 3$ & $1.511(2)$ & $\mathrm{C} 25-\mathrm{H} 25$ & 0.9300 \\
$\mathrm{C} 2-\mathrm{H} 2 \mathrm{~A}$ & 0.9700 & $\mathrm{C} 26-\mathrm{H} 326$ & 0.9300 \\
$\mathrm{C} 2-\mathrm{H} 2 \mathrm{~B}$ & 0.9700 & $\mathrm{O} 24-\mathrm{C} 27$ & $1.420(3)$ \\
$\mathrm{C} 3-\mathrm{N} 4$ & $1.4567(18)$ & $\mathrm{C} 27-\mathrm{H} 27 \mathrm{~A}$ & 0.9600 \\
$\mathrm{C} 3-\mathrm{H} 3 \mathrm{~A}$ & 0.9700 & $\mathrm{C} 27-\mathrm{H} 27 \mathrm{~B}$ & 0.9600 \\
$\mathrm{C} 3-\mathrm{H} 3 \mathrm{~B}$ & 0.9700 & $\mathrm{C} 27-\mathrm{H} 27 \mathrm{C}$ & 0.9600 \\
$\mathrm{~N} 4-\mathrm{C} 21$ & $1.4248(17)$ & $\mathrm{C} 31-\mathrm{O} 31$ & $1.2274(19)$ \\
$\mathrm{N} 4-\mathrm{C} 5$ & $1.4645(19)$ & $\mathrm{C} 31-\mathrm{O} 32$ & $1.283(2)$ \\
$\mathrm{C} 5-\mathrm{C} 6$ & $1.511(2)$ & $\mathrm{C} 31-\mathrm{C} 32$ & $1.492(2)$ \\
$\mathrm{C} 5-\mathrm{H} 5 \mathrm{~A}$ & 0.9700 & $\mathrm{C} 32-\mathrm{C} 33$ & $1.328(2)$
\end{tabular}




\begin{tabular}{|c|c|c|c|}
\hline C5-H5B & 0.9700 & $\mathrm{C} 32-\mathrm{H} 32$ & 0.9300 \\
\hline C6-H6A & 0.9700 & $\mathrm{C} 33-\mathrm{C} 34$ & $1.482(2)$ \\
\hline C6-H6B & 0.9700 & C33-H33 & 0.9300 \\
\hline $\mathrm{C} 21-\mathrm{C} 22$ & $1.383(2)$ & $\mathrm{C} 34-\mathrm{O} 34$ & $1.2355(17)$ \\
\hline $\mathrm{C} 21-\mathrm{C} 26$ & $1.394(2)$ & $\mathrm{C} 34-\mathrm{O} 33$ & $1.2820(17)$ \\
\hline $\mathrm{C} 22-\mathrm{C} 23$ & $1.391(2)$ & $\mathrm{O} 32-\mathrm{H} 33 \mathrm{~A}$ & $1.25(2)$ \\
\hline $\mathrm{C} 22-\mathrm{H} 22$ & 0.9300 & $\mathrm{O} 33-\mathrm{H} 33 \mathrm{~A}$ & $1.17(2)$ \\
\hline $\mathrm{C} 23-\mathrm{C} 24$ & $1.373(2)$ & & \\
\hline $\mathrm{C} 6-\mathrm{N} 1-\mathrm{C} 2$ & $110.57(12)$ & $\mathrm{C} 21-\mathrm{C} 22-\mathrm{C} 23$ & $121.70(14)$ \\
\hline $\mathrm{C} 6-\mathrm{N} 1-\mathrm{H} 11$ & $112.8(11)$ & $\mathrm{C} 21-\mathrm{C} 22-\mathrm{H} 22$ & 119.2 \\
\hline $\mathrm{C} 2-\mathrm{N} 1-\mathrm{H} 11$ & $105.8(11)$ & $\mathrm{C} 23-\mathrm{C} 22-\mathrm{H} 22$ & 119.2 \\
\hline $\mathrm{C} 6-\mathrm{N} 1-\mathrm{H} 12$ & $112.6(11)$ & $\mathrm{C} 24-\mathrm{C} 23-\mathrm{C} 22$ & $120.31(15)$ \\
\hline $\mathrm{C} 2-\mathrm{N} 1-\mathrm{H} 12$ & $106.8(11)$ & $\mathrm{C} 24-\mathrm{C} 23-\mathrm{H} 23$ & 119.8 \\
\hline $\mathrm{H} 11-\mathrm{N} 1-\mathrm{H} 12$ & $107.8(15)$ & $\mathrm{C} 22-\mathrm{C} 23-\mathrm{H} 23$ & 119.8 \\
\hline $\mathrm{N} 1-\mathrm{C} 2-\mathrm{C} 3$ & $110.13(13)$ & $\mathrm{C} 23-\mathrm{C} 24-\mathrm{O} 24$ & $125.33(15)$ \\
\hline $\mathrm{N} 1-\mathrm{C} 2-\mathrm{H} 2 \mathrm{~A}$ & 109.6 & $\mathrm{C} 23-\mathrm{C} 24-\mathrm{C} 25$ & $118.74(14)$ \\
\hline $\mathrm{C} 3-\mathrm{C} 2-\mathrm{H} 2 \mathrm{~A}$ & 109.6 & $\mathrm{O} 24-\mathrm{C} 24-\mathrm{C} 25$ & $115.93(14)$ \\
\hline $\mathrm{N} 1-\mathrm{C} 2-\mathrm{H} 2 \mathrm{~B}$ & 109.6 & $\mathrm{C} 26-\mathrm{C} 25-\mathrm{C} 24$ & $120.98(15)$ \\
\hline $\mathrm{C} 3-\mathrm{C} 2-\mathrm{H} 2 \mathrm{~B}$ & 109.6 & $\mathrm{C} 26-\mathrm{C} 25-\mathrm{H} 25$ & 119.5 \\
\hline $\mathrm{H} 2 \mathrm{~A}-\mathrm{C} 2-\mathrm{H} 2 \mathrm{~B}$ & 108.1 & $\mathrm{C} 24-\mathrm{C} 25-\mathrm{H} 25$ & 119.5 \\
\hline $\mathrm{N} 4-\mathrm{C} 3-\mathrm{C} 2$ & $111.29(13)$ & $\mathrm{C} 25-\mathrm{C} 26-\mathrm{C} 21$ & $121.25(15)$ \\
\hline $\mathrm{N} 4-\mathrm{C} 3-\mathrm{H} 3 \mathrm{~A}$ & 109.4 & $\mathrm{C} 25-\mathrm{C} 26-\mathrm{H} 326$ & 119.4 \\
\hline $\mathrm{C} 2-\mathrm{C} 3-\mathrm{H} 3 \mathrm{~A}$ & 109.4 & $\mathrm{C} 21-\mathrm{C} 26-\mathrm{H} 326$ & 119.4 \\
\hline $\mathrm{N} 4-\mathrm{C} 3-\mathrm{H} 3 \mathrm{~B}$ & 109.4 & $\mathrm{C} 24-\mathrm{O} 24-\mathrm{C} 27$ & $117.53(15)$ \\
\hline $\mathrm{C} 2-\mathrm{C} 3-\mathrm{H} 3 \mathrm{~B}$ & 109.4 & $\mathrm{O} 24-\mathrm{C} 27-\mathrm{H} 27 \mathrm{~A}$ & 109.5 \\
\hline $\mathrm{H} 3 \mathrm{~A}-\mathrm{C} 3-\mathrm{H} 3 \mathrm{~B}$ & 108.0 & $\mathrm{O} 24-\mathrm{C} 27-\mathrm{H} 27 \mathrm{~B}$ & 109.5 \\
\hline $\mathrm{C} 21-\mathrm{N} 4-\mathrm{C} 3$ & $115.42(11)$ & $\mathrm{H} 27 \mathrm{~A}-\mathrm{C} 27-\mathrm{H} 27 \mathrm{~B}$ & 109.5 \\
\hline $\mathrm{C} 21-\mathrm{N} 4-\mathrm{C} 5$ & $114.50(11)$ & $\mathrm{O} 24-\mathrm{C} 27-\mathrm{H} 27 \mathrm{C}$ & 109.5 \\
\hline $\mathrm{C} 3-\mathrm{N} 4-\mathrm{C} 5$ & $109.87(12)$ & $\mathrm{H} 27 \mathrm{~A}-\mathrm{C} 27-\mathrm{H} 27 \mathrm{C}$ & 109.5 \\
\hline $\mathrm{N} 4-\mathrm{C} 5-\mathrm{C} 6$ & $111.79(12)$ & $\mathrm{H} 27 \mathrm{~B}-\mathrm{C} 27-\mathrm{H} 27 \mathrm{C}$ & 109.5 \\
\hline $\mathrm{N} 4-\mathrm{C} 5-\mathrm{H} 5 \mathrm{~A}$ & 109.3 & $\mathrm{O} 31-\mathrm{C} 31-\mathrm{O} 32$ & $121.90(14)$ \\
\hline $\mathrm{C} 6-\mathrm{C} 5-\mathrm{H} 5 \mathrm{~A}$ & 109.3 & $\mathrm{O} 31-\mathrm{C} 31-\mathrm{C} 32$ & $118.52(15)$ \\
\hline $\mathrm{N} 4-\mathrm{C} 5-\mathrm{H} 5 \mathrm{~B}$ & 109.3 & $\mathrm{O} 32-\mathrm{C} 31-\mathrm{C} 32$ & $119.57(14)$ \\
\hline $\mathrm{C} 6-\mathrm{C} 5-\mathrm{H} 5 \mathrm{~B}$ & 109.3 & $\mathrm{C} 33-\mathrm{C} 32-\mathrm{C} 31$ & $130.65(15)$ \\
\hline $\mathrm{H} 5 \mathrm{~A}-\mathrm{C} 5-\mathrm{H} 5 \mathrm{~B}$ & 107.9 & $\mathrm{C} 33-\mathrm{C} 32-\mathrm{H} 32$ & 114.7 \\
\hline $\mathrm{N} 1-\mathrm{C} 6-\mathrm{C} 5$ & $109.95(13)$ & $\mathrm{C} 31-\mathrm{C} 32-\mathrm{H} 32$ & 114.7 \\
\hline $\mathrm{N} 1-\mathrm{C} 6-\mathrm{H} 6 \mathrm{~A}$ & 109.7 & $\mathrm{C} 32-\mathrm{C} 33-\mathrm{C} 34$ & $130.48(13)$ \\
\hline $\mathrm{C} 5-\mathrm{C} 6-\mathrm{H} 6 \mathrm{~A}$ & 109.7 & $\mathrm{C} 32-\mathrm{C} 33-\mathrm{H} 33$ & 114.8 \\
\hline $\mathrm{N} 1-\mathrm{C} 6-\mathrm{H} 6 \mathrm{~B}$ & 109.7 & $\mathrm{C} 34-\mathrm{C} 33-\mathrm{H} 33$ & 114.8 \\
\hline $\mathrm{C} 5-\mathrm{C} 6-\mathrm{H} 6 \mathrm{~B}$ & 109.7 & $\mathrm{O} 34-\mathrm{C} 34-\mathrm{O} 33$ & $122.51(14)$ \\
\hline $\mathrm{H} 6 \mathrm{~A}-\mathrm{C} 6-\mathrm{H} 6 \mathrm{~B}$ & 108.2 & $\mathrm{O} 34-\mathrm{C} 34-\mathrm{C} 33$ & $117.29(13)$ \\
\hline $\mathrm{C} 22-\mathrm{C} 21-\mathrm{C} 26$ & $117.01(13)$ & $\mathrm{O} 33-\mathrm{C} 34-\mathrm{C} 33$ & $120.20(12)$ \\
\hline $\mathrm{C} 22-\mathrm{C} 21-\mathrm{N} 4$ & $122.86(13)$ & $\mathrm{C} 31-\mathrm{O} 32-\mathrm{H} 33 \mathrm{~A}$ & $111.7(9)$ \\
\hline $\mathrm{C} 26-\mathrm{C} 21-\mathrm{N} 4$ & $120.09(13)$ & $\mathrm{C} 34-\mathrm{O} 33-\mathrm{H} 33 \mathrm{~A}$ & $111.5(10)$ \\
\hline $\mathrm{C} 6-\mathrm{N} 1-\mathrm{C} 2-\mathrm{C} 3$ & $-57.07(18)$ & $\mathrm{C} 22-\mathrm{C} 23-\mathrm{C} 24-\mathrm{O} 24$ & $-179.65(16)$ \\
\hline $\mathrm{N} 1-\mathrm{C} 2-\mathrm{C} 3-\mathrm{N} 4$ & $57.68(18)$ & $\mathrm{C} 22-\mathrm{C} 23-\mathrm{C} 24-\mathrm{C} 25$ & $1.0(3)$ \\
\hline
\end{tabular}




$\begin{array}{ll}\mathrm{C} 2-\mathrm{C} 3-\mathrm{N} 4-\mathrm{C} 21 & 171.43(13) \\ \mathrm{C} 2-\mathrm{C} 3-\mathrm{N} 4-\mathrm{C} 5 & -57.26(17) \\ \mathrm{C} 21-\mathrm{N} 4-\mathrm{C} 5-\mathrm{C} 6 & -171.06(12) \\ \mathrm{C} 3-\mathrm{N} 4-\mathrm{C} 5-\mathrm{C} 6 & 57.15(17) \\ \mathrm{C} 2-\mathrm{N} 1-\mathrm{C} 6-\mathrm{C} 5 & 56.57(17) \\ \mathrm{N} 4-\mathrm{C} 5-\mathrm{C} 6-\mathrm{N} 1 & -57.02(18) \\ \mathrm{C} 3-\mathrm{N} 4-\mathrm{C} 21-\mathrm{C} 22 & -3.3(2) \\ \mathrm{C} 5-\mathrm{N} 4-\mathrm{C} 21-\mathrm{C} 22 & -132.32(16) \\ \mathrm{C} 3-\mathrm{N} 4-\mathrm{C} 21-\mathrm{C} 26 & 178.91(14) \\ \mathrm{C} 5-\mathrm{N} 4-\mathrm{C} 21-\mathrm{C} 26 & 49.85(18) \\ \mathrm{C} 26-\mathrm{C} 21-\mathrm{C} 22-\mathrm{C} 23 & 0.6(2) \\ \mathrm{N} 4-\mathrm{C} 21-\mathrm{C} 22-\mathrm{C} 23 & -177.33(14) \\ \mathrm{C} 21-\mathrm{C} 22-\mathrm{C} 23-\mathrm{C} 24 & -1.3(3)\end{array}$

$\mathrm{C} 23-\mathrm{C} 24-\mathrm{C} 25-\mathrm{C} 26$
$\mathrm{O} 24-\mathrm{C} 24-\mathrm{C} 25-\mathrm{C} 26$
$\mathrm{C} 24-\mathrm{C} 25-\mathrm{C} 26-\mathrm{C} 21$
$\mathrm{C} 22-\mathrm{C} 21-\mathrm{C} 26-\mathrm{C} 25$
$\mathrm{~N} 4-\mathrm{C} 21-\mathrm{C} 26-\mathrm{C} 25$
$\mathrm{C} 23-\mathrm{C} 24-\mathrm{O} 24-\mathrm{C} 27$
$\mathrm{C} 25-\mathrm{C} 24-\mathrm{O} 24-\mathrm{C} 27$
$\mathrm{O} 31-\mathrm{C} 31-\mathrm{C} 32-\mathrm{C} 33$
$\mathrm{O} 32-\mathrm{C} 31-\mathrm{C} 32-\mathrm{C} 33$
$\mathrm{C} 31-\mathrm{C} 32-\mathrm{C} 33-\mathrm{C} 34$
$\mathrm{C} 32-\mathrm{C} 33-\mathrm{C} 34-\mathrm{O} 34$
$\mathrm{C} 32-\mathrm{C} 33-\mathrm{C} 34-\mathrm{O} 33$

$-0.1(3)$

$-179.42(16)$

$-0.7(3)$

$0.4(2)$

$178.39(14)$

$-4.6(3)$

$174.67(18)$

$-173.24(17)$

$6.0(2)$

$-0.5(3)$

$173.69(16)$

$-6.1(2)$

Hydrogen-bond geometry $\left(A,{ }^{\circ}\right)$

\begin{tabular}{lllll}
\hline$D-\mathrm{H} \cdots A$ & $D-\mathrm{H}$ & $\mathrm{H} \cdots A$ & $D \cdots A$ & $D-\mathrm{H} \cdots A$ \\
\hline $\mathrm{O} 33-\mathrm{H} 33 A \cdots \mathrm{O} 32$ & $1.167(18)$ & $1.247(18)$ & $2.4121(16)$ & $175(2)$ \\
$\mathrm{N} 1-\mathrm{H} 11 \cdots \mathrm{O} 31$ & $0.915(17)$ & $2.126(16)$ & $2.9309(19)$ & $146.2(15)$ \\
$\mathrm{N} 1-\mathrm{H} 11 \cdots \mathrm{O} 32$ & $0.915(17)$ & $2.296(17)$ & $3.0798(18)$ & $143.5(14)$ \\
$\mathrm{N} 1-\mathrm{H} 12 \cdots \mathrm{O} 34^{\mathrm{i}}$ & $0.919(18)$ & $1.881(18)$ & $2.7563(17)$ & $158.5(17)$ \\
$\mathrm{C} 2-\mathrm{H} 2 A \cdots \mathrm{O} 34^{\mathrm{ii}}$ & 0.97 & 2.56 & $3.363(2)$ & 140
\end{tabular}

Symmetry codes: (i) $x-1, y, z$; (ii) $-x+2,-y+1,-z+1$.

4-(4-Methoxyphenyl)piperazin-1-ium trichloroacetate (XI)

Crystal data

$\mathrm{C}_{11} \mathrm{H}_{17} \mathrm{~N}_{2} \mathrm{O}^{+} \cdot \mathrm{C}_{2} \mathrm{Cl}_{3} \mathrm{O}_{2}^{-}$

$M_{r}=355.64$

Orthorhombic, $P c a 2_{1}$

$a=10.6117(11) \AA$

$b=13.808(1) \AA$

$c=10.9137(8) \AA$

$V=1599.1(2) \AA^{3}$

$Z=4$

$F(000)=736$

\section{Data collection}

Oxford Diffraction Xcalibur with Sapphire CCD diffractometer

Radiation source: Enhance (Mo) X-ray Source

Graphite monochromator

$\omega$ scans

Absorption correction: multi-scan

(CrysAlis RED; Oxford Diffraction, 2009)

$T_{\text {min }}=0.476, T_{\max }=0.892$
$D_{\mathrm{x}}=1.477 \mathrm{Mg} \mathrm{m}^{-3}$

Mo $K \alpha$ radiation, $\lambda=0.71073 \AA$

Cell parameters from 2428 reflections

$\theta=3.0-27.7^{\circ}$

$\mu=0.58 \mathrm{~mm}^{-1}$

$T=296 \mathrm{~K}$

Block, colourless

$0.48 \times 0.48 \times 0.20 \mathrm{~mm}$

6173 measured reflections

2428 independent reflections

2278 reflections with $I>2 \sigma(I)$

$R_{\text {int }}=0.027$

$\theta_{\text {max }}=27.7^{\circ}, \theta_{\min }=3.0^{\circ}$

$h=-5 \rightarrow 13$

$k=-16 \rightarrow 17$

$l=-14 \rightarrow 5$ 


\section{Refinement}

Refinement on $F^{2}$

Least-squares matrix: full

$R\left[F^{2}>2 \sigma\left(F^{2}\right)\right]=0.032$

$w R\left(F^{2}\right)=0.086$

$S=1.08$

2428 reflections

198 parameters

1 restraint

Primary atom site location: difference Fourier map

Hydrogen site location: mixed
$\mathrm{H}$ atoms treated by a mixture of independent and constrained refinement

$w=1 /\left[\sigma^{2}\left(F_{\mathrm{o}}^{2}\right)+(0.0532 P)^{2}+0.3843 P\right]$ where $P=\left(F_{\mathrm{o}}^{2}+2 F_{\mathrm{c}}^{2}\right) / 3$

$(\Delta / \sigma)_{\max }<0.001$

$\Delta \rho_{\max }=0.25 \mathrm{e} \AA^{-3}$

$\Delta \rho_{\min }=-0.30$ e $\AA^{-3}$

Extinction correction: SHELXL, $\mathrm{Fc}^{*}=\mathrm{kFc}\left[1+0.001 \mathrm{xFc}^{2} \lambda^{3} / \sin (2 \theta)\right]^{-1 / 4}$

Extinction coefficient: 0.023 (2)

Absolute structure: Classical Flack method preferred over Parsons because s.u. lower Absolute structure parameter: 0.11 (7)

Special details

Experimental. Compound (XI). IR $\left(\mathrm{KBr}, \mathrm{cm}^{-1}\right) 3073\left(\mathrm{NH}_{2}\right), 2829\left(\mathrm{OCH}_{3}\right), 1561(\mathrm{COO})$. NMR $\left.\left(\mathrm{CDCl}_{3}\right) \delta\left({ }^{1} \mathrm{H}\right)\right) 3.07(\mathrm{~m}$, $4 \mathrm{H}$, piperazine), $3.19\left(\mathrm{~m}, 4 \mathrm{H}\right.$, piperazine), $3.77\left(\mathrm{~s}, 3 \mathrm{H}, \mathrm{OCH}_{3}\right), 6.89(\mathrm{~m}, 4 \mathrm{H}$, methoxyphenyl).

Geometry. All esds (except the esd in the dihedral angle between two 1.s. planes) are estimated using the full covariance matrix. The cell esds are taken into account individually in the estimation of esds in distances, angles and torsion angles; correlations between esds in cell parameters are only used when they are defined by crystal symmetry. An approximate (isotropic) treatment of cell esds is used for estimating esds involving l.s. planes.

Fractional atomic coordinates and isotropic or equivalent isotropic displacement parameters $\left(\AA^{2}\right)$

\begin{tabular}{lllll}
\hline & $x$ & $y$ & $z$ & $U_{\text {iso }} * / U_{\text {eq }}$ \\
\hline $\mathrm{N} 1$ & $0.1394(2)$ & $0.44188(17)$ & $0.2473(3)$ & $0.0301(5)$ \\
$\mathrm{H} 11$ & $0.168(3)$ & $0.495(3)$ & $0.204(3)$ & $0.036^{*}$ \\
$\mathrm{H} 12$ & $0.061(3)$ & $0.426(3)$ & $0.206(4)$ & $0.036^{*}$ \\
$\mathrm{C} 2$ & $0.1158(3)$ & $0.4667(2)$ & $0.3775(3)$ & $0.0343(7)$ \\
$\mathrm{H} 2 \mathrm{~A}$ & 0.0542 & 0.5185 & 0.3822 & $0.041^{*}$ \\
$\mathrm{H} 2 \mathrm{~B}$ & 0.1933 & 0.4895 & 0.4148 & $0.041^{*}$ \\
$\mathrm{C} 3$ & $0.0680(3)$ & $0.37961(19)$ & $0.4468(3)$ & $0.0320(6)$ \\
$\mathrm{H} 3 \mathrm{~A}$ & 0.0546 & 0.3967 & 0.5320 & $0.038^{*}$ \\
$\mathrm{H} 3 \mathrm{~B}$ & -0.0121 & 0.3591 & 0.4126 & $0.038^{*}$ \\
$\mathrm{~N} 4$ & $0.1586(2)$ & $0.30039(15)$ & $0.4389(2)$ & $0.0261(5)$ \\
$\mathrm{C} 5$ & $0.1815(3)$ & $0.2750(2)$ & $0.3106(3)$ & $0.0311(6)$ \\
$\mathrm{H} 5 \mathrm{~A}$ & 0.1037 & 0.2523 & 0.2738 & $0.037^{*}$ \\
$\mathrm{H} 5 \mathrm{~B}$ & 0.2424 & 0.2227 & 0.3067 & $0.037^{*}$ \\
$\mathrm{C} 6$ & $0.2304(2)$ & $0.3607(2)$ & $0.2392(3)$ & $0.0324(6)$ \\
$\mathrm{H} 6 \mathrm{~A}$ & 0.3112 & 0.3808 & 0.2721 & $0.039^{*}$ \\
$\mathrm{H} 6 \mathrm{~B}$ & 0.2423 & 0.3426 & 0.1541 & $0.039^{*}$ \\
$\mathrm{C} 21$ & $0.1350(2)$ & $0.22071(19)$ & $0.5194(3)$ & $0.0269(6)$ \\
$\mathrm{C} 22$ & $0.0345(3)$ & $0.2170(2)$ & $0.5993(3)$ & $0.0347(6)$ \\
$\mathrm{H} 22$ & -0.0265 & 0.2653 & 0.5959 & $0.042^{*}$ \\
$\mathrm{C} 23$ & $0.0216(3)$ & $0.1433(2)$ & $0.6846(3)$ & $0.0396(7)$ \\
$\mathrm{H} 23$ & -0.0468 & 0.1430 & 0.7379 & $0.048^{*}$ \\
$\mathrm{C} 24$ & $0.1103(3)$ & $0.0700(2)$ & $0.6906(3)$ & $0.0398(7)$ \\
$\mathrm{C} 25$ & $0.2088(3)$ & $0.0707(2)$ & $0.6074(4)$ & $0.0388(7)$ \\
H25 & 0.2671 & 0.0205 & 0.6083 & $0.047^{*}$
\end{tabular}




\begin{tabular}{lllll}
$\mathrm{C} 26$ & $0.2220(3)$ & $0.14432(19)$ & $0.5235(3)$ & $0.0324(6)$ \\
$\mathrm{H} 26$ & 0.2892 & 0.1434 & 0.4689 & $0.039^{*}$ \\
$\mathrm{O} 24$ & $0.1108(3)$ & $-0.00368(18)$ & $0.7747(3)$ & $0.0587(8)$ \\
$\mathrm{C} 27$ & $0.0095(6)$ & $-0.0086(4)$ & $0.8579(5)$ & $0.0733(13)$ \\
$\mathrm{H} 27 \mathrm{~A}$ & 0.0234 & -0.0608 & 0.9145 & $0.110^{*}$ \\
$\mathrm{H} 27 \mathrm{~B}$ & -0.0675 & -0.0197 & 0.8138 & $0.110^{*}$ \\
$\mathrm{H} 27 \mathrm{C}$ & 0.0035 & 0.0513 & 0.9022 & $0.110^{*}$ \\
$\mathrm{C} 31$ & $0.3163(2)$ & $0.61757(18)$ & $0.1132(3)$ & $0.0266(5)$ \\
$\mathrm{O} 31$ & $0.20314(18)$ & $0.60081(16)$ & $0.1045(3)$ & $0.0444(6)$ \\
O32 & $0.39463(19)$ & $0.57402(18)$ & $0.1749(3)$ & $0.0464(6)$ \\
C32 & $0.3653(3)$ & $0.7076(2)$ & $0.0383(3)$ & $0.0304(6)$ \\
C11 & $0.52532(7)$ & $0.69160(6)$ & $-0.00662(9)$ & $0.0426(2)$ \\
C12 & $0.35730(10)$ & $0.80958(5)$ & $0.13523(10)$ & $0.0505(3)$ \\
C13 & $0.27618(8)$ & $0.72790(7)$ & $-0.09480(9)$ & $0.0540(3)$ \\
\hline
\end{tabular}

Atomic displacement parameters $\left(\AA^{2}\right)$

\begin{tabular}{lllllll}
\hline & $U^{11}$ & $U^{22}$ & $U^{33}$ & $U^{12}$ & $U^{13}$ & $U^{23}$ \\
\hline $\mathrm{N} 1$ & $0.0286(11)$ & $0.0228(11)$ & $0.0389(15)$ & $-0.0020(8)$ & $-0.0003(11)$ & $0.0038(11)$ \\
$\mathrm{C} 2$ & $0.0433(14)$ & $0.0199(13)$ & $0.0398(18)$ & $0.0022(11)$ & $-0.0004(14)$ & $-0.0032(12)$ \\
$\mathrm{C} 3$ & $0.0354(13)$ & $0.0242(13)$ & $0.0364(16)$ & $0.0059(11)$ & $0.0060(12)$ & $-0.0015(12)$ \\
$\mathrm{N} 4$ & $0.0304(11)$ & $0.0178(10)$ & $0.0300(13)$ & $0.0008(8)$ & $0.0049(9)$ & $-0.0026(9)$ \\
$\mathrm{C} 5$ & $0.0370(14)$ & $0.0234(13)$ & $0.0331(15)$ & $0.0034(11)$ & $0.0027(13)$ & $-0.0025(12)$ \\
$\mathrm{C} 6$ & $0.0308(14)$ & $0.0307(14)$ & $0.0356(16)$ & $0.0041(10)$ & $0.0064(13)$ & $0.0002(13)$ \\
$\mathrm{C} 21$ & $0.0295(11)$ & $0.0196(11)$ & $0.0316(16)$ & $-0.0051(9)$ & $0.0001(11)$ & $-0.0022(11)$ \\
$\mathrm{C} 22$ & $0.0335(13)$ & $0.0295(13)$ & $0.0411(18)$ & $-0.0016(11)$ & $0.0036(13)$ & $-0.0032(13)$ \\
$\mathrm{C} 23$ & $0.0455(15)$ & $0.0342(15)$ & $0.0392(19)$ & $-0.0102(13)$ & $0.0117(14)$ & $-0.0011(14)$ \\
$\mathrm{C} 24$ & $0.0628(19)$ & $0.0206(12)$ & $0.0361(18)$ & $-0.0128(13)$ & $-0.0005(15)$ & $-0.0005(13)$ \\
$\mathrm{C} 25$ & $0.0487(15)$ & $0.0198(12)$ & $0.048(2)$ & $0.0005(11)$ & $-0.0002(16)$ & $0.0004(13)$ \\
$\mathrm{C} 26$ & $0.0336(13)$ & $0.0229(13)$ & $0.0408(18)$ & $-0.0009(10)$ & $0.0034(12)$ & $-0.0015(13)$ \\
$\mathrm{O} 24$ & $0.0943(19)$ & $0.0332(12)$ & $0.0485(17)$ & $-0.0085(13)$ & $0.0079(15)$ & $0.0129(13)$ \\
$\mathrm{C} 27$ & $0.095(3)$ & $0.064(3)$ & $0.061(3)$ & $-0.028(2)$ & $0.008(3)$ & $0.023(2)$ \\
$\mathrm{C} 31$ & $0.0303(12)$ & $0.0190(11)$ & $0.0305(15)$ & $-0.0017(9)$ & $0.0033(12)$ & $0.0005(11)$ \\
O31 & $0.0301(9)$ & $0.0391(11)$ & $0.0640(17)$ & $-0.0070(8)$ & $-0.0039(11)$ & $0.0214(12)$ \\
O32 & $0.0328(11)$ & $0.0421(12)$ & $0.0643(18)$ & $-0.0046(9)$ & $-0.0072(11)$ & $0.0244(12)$ \\
C32 & $0.0320(12)$ & $0.0260(13)$ & $0.0332(15)$ & $-0.0049(10)$ & $-0.0009(12)$ & $0.0029(12)$ \\
C11 & $0.0343(3)$ & $0.0457(4)$ & $0.0478(5)$ & $-0.0125(3)$ & $0.0085(3)$ & $-0.0007(4)$ \\
C12 & $0.0714(6)$ & $0.0236(3)$ & $0.0565(6)$ & $-0.0017(3)$ & $0.0089(5)$ & $-0.0065(4)$ \\
C13 & $0.0514(5)$ & $0.0652(6)$ & $0.0455(5)$ & $-0.0134(4)$ & $-0.0124(4)$ & $0.0233(5)$ \\
& & & & & & \\
\hline & & & & & &
\end{tabular}

Geometric parameters $\left(\AA,{ }^{\circ}\right)$

\begin{tabular}{llll}
\hline $\mathrm{N} 1-\mathrm{C} 6$ & $1.482(3)$ & $\mathrm{C} 22-\mathrm{C} 23$ & $1.386(5)$ \\
$\mathrm{N} 1-\mathrm{C} 2$ & $1.484(4)$ & $\mathrm{C} 22-\mathrm{H} 22$ & 0.9300 \\
$\mathrm{~N} 1-\mathrm{H} 11$ & $0.92(4)$ & $\mathrm{C} 23-\mathrm{C} 24$ & $1.383(5)$ \\
$\mathrm{N} 1-\mathrm{H} 12$ & $0.97(3)$ & $\mathrm{C} 23-\mathrm{H} 23$ & 0.9300 \\
$\mathrm{C} 2-\mathrm{C} 3$ & $1.509(4)$ & $\mathrm{C} 24-\mathrm{O} 24$ & $1.370(4)$ \\
$\mathrm{C} 2-\mathrm{H} 2 \mathrm{~A}$ & 0.9700 & $\mathrm{C} 24-\mathrm{C} 25$ & $1.385(5)$
\end{tabular}




\begin{tabular}{|c|c|c|c|}
\hline $\mathrm{C} 2-\mathrm{H} 2 \mathrm{~B}$ & 0.9700 & $\mathrm{C} 25-\mathrm{C} 26$ & $1.375(4)$ \\
\hline $\mathrm{C} 3-\mathrm{N} 4$ & $1.459(3)$ & $\mathrm{C} 25-\mathrm{H} 25$ & 0.9300 \\
\hline $\mathrm{C} 3-\mathrm{H} 3 \mathrm{~A}$ & 0.9700 & $\mathrm{C} 26-\mathrm{H} 26$ & 0.9300 \\
\hline $\mathrm{C} 3-\mathrm{H} 3 \mathrm{~B}$ & 0.9700 & $\mathrm{O} 24-\mathrm{C} 27$ & $1.410(6)$ \\
\hline $\mathrm{N} 4-\mathrm{C} 21$ & $1.430(4)$ & $\mathrm{C} 27-\mathrm{H} 27 \mathrm{~A}$ & 0.9600 \\
\hline $\mathrm{N} 4-\mathrm{C} 5$ & $1.464(4)$ & $\mathrm{C} 27-\mathrm{H} 27 \mathrm{~B}$ & 0.9600 \\
\hline $\mathrm{C} 5-\mathrm{C} 6$ & $1.509(4)$ & $\mathrm{C} 27-\mathrm{H} 27 \mathrm{C}$ & 0.9600 \\
\hline $\mathrm{C} 5-\mathrm{H} 5 \mathrm{~A}$ & 0.9700 & $\mathrm{C} 31-\mathrm{O} 31$ & $1.227(3)$ \\
\hline C5-H5B & 0.9700 & $\mathrm{C} 31-\mathrm{O} 32$ & $1.227(4)$ \\
\hline C6-H6A & 0.9700 & $\mathrm{C} 31-\mathrm{C} 32$ & $1.576(4)$ \\
\hline C6-H6B & 0.9700 & $\mathrm{C} 32-\mathrm{Cl} 3$ & $1.756(3)$ \\
\hline $\mathrm{C} 21-\mathrm{C} 22$ & $1.379(4)$ & $\mathrm{C} 32-\mathrm{Cl} 2$ & $1.763(3)$ \\
\hline $\mathrm{C} 21-\mathrm{C} 26$ & $1.402(4)$ & $\mathrm{C} 32-\mathrm{Cl1}$ & $1.781(3)$ \\
\hline $\mathrm{C} 6-\mathrm{N} 1-\mathrm{C} 2$ & $110.0(2)$ & $\mathrm{C} 22-\mathrm{C} 21-\mathrm{N} 4$ & $123.5(3)$ \\
\hline $\mathrm{C} 6-\mathrm{N} 1-\mathrm{H} 11$ & $111(2)$ & $\mathrm{C} 26-\mathrm{C} 21-\mathrm{N} 4$ & $118.9(2)$ \\
\hline $\mathrm{C} 2-\mathrm{N} 1-\mathrm{H} 11$ & $111(2)$ & $\mathrm{C} 21-\mathrm{C} 22-\mathrm{C} 23$ & $121.9(3)$ \\
\hline $\mathrm{C} 6-\mathrm{N} 1-\mathrm{H} 12$ & $111(2)$ & $\mathrm{C} 21-\mathrm{C} 22-\mathrm{H} 22$ & 119.0 \\
\hline $\mathrm{C} 2-\mathrm{N} 1-\mathrm{H} 12$ & $111(2)$ & $\mathrm{C} 23-\mathrm{C} 22-\mathrm{H} 22$ & 119.0 \\
\hline $\mathrm{H} 11-\mathrm{N} 1-\mathrm{H} 12$ & $103(3)$ & $\mathrm{C} 24-\mathrm{C} 23-\mathrm{C} 22$ & $120.1(3)$ \\
\hline $\mathrm{N} 1-\mathrm{C} 2-\mathrm{C} 3$ & $110.6(2)$ & $\mathrm{C} 24-\mathrm{C} 23-\mathrm{H} 23$ & 119.9 \\
\hline $\mathrm{N} 1-\mathrm{C} 2-\mathrm{H} 2 \mathrm{~A}$ & 109.5 & $\mathrm{C} 22-\mathrm{C} 23-\mathrm{H} 23$ & 119.9 \\
\hline $\mathrm{C} 3-\mathrm{C} 2-\mathrm{H} 2 \mathrm{~A}$ & 109.5 & $\mathrm{O} 24-\mathrm{C} 24-\mathrm{C} 23$ & $125.3(3)$ \\
\hline $\mathrm{N} 1-\mathrm{C} 2-\mathrm{H} 2 \mathrm{~B}$ & 109.5 & $\mathrm{O} 24-\mathrm{C} 24-\mathrm{C} 25$ & $116.2(3)$ \\
\hline $\mathrm{C} 3-\mathrm{C} 2-\mathrm{H} 2 \mathrm{~B}$ & 109.5 & $\mathrm{C} 23-\mathrm{C} 24-\mathrm{C} 25$ & $118.5(3)$ \\
\hline $\mathrm{H} 2 \mathrm{~A}-\mathrm{C} 2-\mathrm{H} 2 \mathrm{~B}$ & 108.1 & $\mathrm{C} 26-\mathrm{C} 25-\mathrm{C} 24$ & $121.3(3)$ \\
\hline $\mathrm{N} 4-\mathrm{C} 3-\mathrm{C} 2$ & $110.3(2)$ & $\mathrm{C} 26-\mathrm{C} 25-\mathrm{H} 25$ & 119.4 \\
\hline $\mathrm{N} 4-\mathrm{C} 3-\mathrm{H} 3 \mathrm{~A}$ & 109.6 & $\mathrm{C} 24-\mathrm{C} 25-\mathrm{H} 25$ & 119.4 \\
\hline $\mathrm{C} 2-\mathrm{C} 3-\mathrm{H} 3 \mathrm{~A}$ & 109.6 & $\mathrm{C} 25-\mathrm{C} 26-\mathrm{C} 21$ & $120.7(3)$ \\
\hline $\mathrm{N} 4-\mathrm{C} 3-\mathrm{H} 3 \mathrm{~B}$ & 109.6 & $\mathrm{C} 25-\mathrm{C} 26-\mathrm{H} 26$ & 119.7 \\
\hline $\mathrm{C} 2-\mathrm{C} 3-\mathrm{H} 3 \mathrm{~B}$ & 109.6 & $\mathrm{C} 21-\mathrm{C} 26-\mathrm{H} 26$ & 119.7 \\
\hline $\mathrm{H} 3 \mathrm{~A}-\mathrm{C} 3-\mathrm{H} 3 \mathrm{~B}$ & 108.1 & $\mathrm{C} 24-\mathrm{O} 24-\mathrm{C} 27$ & $117.7(3)$ \\
\hline $\mathrm{C} 21-\mathrm{N} 4-\mathrm{C} 3$ & $115.2(2)$ & $\mathrm{O} 24-\mathrm{C} 27-\mathrm{H} 27 \mathrm{~A}$ & 109.5 \\
\hline $\mathrm{C} 21-\mathrm{N} 4-\mathrm{C} 5$ & $115.6(2)$ & $\mathrm{O} 24-\mathrm{C} 27-\mathrm{H} 27 \mathrm{~B}$ & 109.5 \\
\hline $\mathrm{C} 3-\mathrm{N} 4-\mathrm{C} 5$ & $110.2(2)$ & $\mathrm{H} 27 \mathrm{~A}-\mathrm{C} 27-\mathrm{H} 27 \mathrm{~B}$ & 109.5 \\
\hline $\mathrm{N} 4-\mathrm{C} 5-\mathrm{C} 6$ & $111.3(2)$ & $\mathrm{O} 24-\mathrm{C} 27-\mathrm{H} 27 \mathrm{C}$ & 109.5 \\
\hline $\mathrm{N} 4-\mathrm{C} 5-\mathrm{H} 5 \mathrm{~A}$ & 109.4 & $\mathrm{H} 27 \mathrm{~A}-\mathrm{C} 27-\mathrm{H} 27 \mathrm{C}$ & 109.5 \\
\hline $\mathrm{C} 6-\mathrm{C} 5-\mathrm{H} 5 \mathrm{~A}$ & 109.4 & $\mathrm{H} 27 \mathrm{~B}-\mathrm{C} 27-\mathrm{H} 27 \mathrm{C}$ & 109.5 \\
\hline $\mathrm{N} 4-\mathrm{C} 5-\mathrm{H} 5 \mathrm{~B}$ & 109.4 & $\mathrm{O} 31-\mathrm{C} 31-\mathrm{O} 32$ & $127.8(3)$ \\
\hline $\mathrm{C} 6-\mathrm{C} 5-\mathrm{H} 5 \mathrm{~B}$ & 109.4 & $\mathrm{O} 31-\mathrm{C} 31-\mathrm{C} 32$ & $115.6(2)$ \\
\hline $\mathrm{H} 5 \mathrm{~A}-\mathrm{C} 5-\mathrm{H} 5 \mathrm{~B}$ & 108.0 & $\mathrm{O} 32-\mathrm{C} 31-\mathrm{C} 32$ & $116.6(2)$ \\
\hline $\mathrm{N} 1-\mathrm{C} 6-\mathrm{C} 5$ & $109.8(2)$ & $\mathrm{C} 31-\mathrm{C} 32-\mathrm{Cl} 3$ & $112.15(19)$ \\
\hline $\mathrm{N} 1-\mathrm{C} 6-\mathrm{H} 6 \mathrm{~A}$ & 109.7 & $\mathrm{C} 31-\mathrm{C} 32-\mathrm{Cl} 2$ & $107.6(2)$ \\
\hline $\mathrm{C} 5-\mathrm{C} 6-\mathrm{H} 6 \mathrm{~A}$ & 109.7 & $\mathrm{Cl} 3-\mathrm{C} 32-\mathrm{Cl} 2$ & $110.05(16)$ \\
\hline $\mathrm{N} 1-\mathrm{C} 6-\mathrm{H} 6 \mathrm{~B}$ & 109.7 & $\mathrm{C} 31-\mathrm{C} 32-\mathrm{Cl} 1$ & $111.06(19)$ \\
\hline $\mathrm{C} 5-\mathrm{C} 6-\mathrm{H} 6 \mathrm{~B}$ & 109.7 & $\mathrm{Cl} 3-\mathrm{C} 32-\mathrm{Cl} 1$ & $107.82(18)$ \\
\hline $\mathrm{H} 6 \mathrm{~A}-\mathrm{C} 6-\mathrm{H} 6 \mathrm{~B}$ & 108.2 & $\mathrm{Cl} 2-\mathrm{C} 32-\mathrm{Cl} 1$ & $108.08(15)$ \\
\hline $\mathrm{C} 22-\mathrm{C} 21-\mathrm{C} 26$ & $117.4(3)$ & & \\
\hline
\end{tabular}




\begin{tabular}{llll}
$\mathrm{C} 6-\mathrm{N} 1-\mathrm{C} 2-\mathrm{C} 3$ & $-57.4(3)$ & $\mathrm{C} 22-\mathrm{C} 23-\mathrm{C} 24-\mathrm{O} 24$ & $176.6(3)$ \\
$\mathrm{N} 1-\mathrm{C} 2-\mathrm{C} 3-\mathrm{N} 4$ & $58.2(3)$ & $\mathrm{C} 22-\mathrm{C} 23-\mathrm{C} 24-\mathrm{C} 25$ & $-2.1(5)$ \\
$\mathrm{C} 2-\mathrm{C} 3-\mathrm{N} 4-\mathrm{C} 21$ & $168.8(3)$ & $\mathrm{O} 24-\mathrm{C} 24-\mathrm{C} 25-\mathrm{C} 26$ & $-176.2(3)$ \\
$\mathrm{C} 2-\mathrm{C} 3-\mathrm{N} 4-\mathrm{C} 5$ & $-58.2(3)$ & $\mathrm{C} 23-\mathrm{C} 24-\mathrm{C} 25-\mathrm{C} 26$ & $2.6(5)$ \\
$\mathrm{C} 21-\mathrm{N} 4-\mathrm{C} 5-\mathrm{C} 6$ & $-168.7(2)$ & $\mathrm{C} 24-\mathrm{C} 25-\mathrm{C} 26-\mathrm{C} 21$ & $-0.4(5)$ \\
$\mathrm{C} 3-\mathrm{N} 4-\mathrm{C} 5-\mathrm{C} 6$ & $58.5(3)$ & $\mathrm{C} 22-\mathrm{C} 21-\mathrm{C} 26-\mathrm{C} 25$ & $-2.3(4)$ \\
$\mathrm{C} 2-\mathrm{N} 1-\mathrm{C} 6-\mathrm{C} 5$ & $56.6(3)$ & $\mathrm{N} 4-\mathrm{C} 21-\mathrm{C} 26-\mathrm{C} 25$ & $174.1(3)$ \\
$\mathrm{N} 4-\mathrm{C} 5-\mathrm{C} 6-\mathrm{N} 1$ & $-57.6(3)$ & $\mathrm{C} 23-\mathrm{C} 24-\mathrm{O} 24-\mathrm{C} 27$ & $3.5(5)$ \\
$\mathrm{C} 3-\mathrm{N} 4-\mathrm{C} 21-\mathrm{C} 22$ & $0.2(4)$ & $\mathrm{C} 25-\mathrm{C} 24-\mathrm{O} 24-\mathrm{C} 27$ & $-177.7(4)$ \\
$\mathrm{C} 5-\mathrm{N} 4-\mathrm{C} 21-\mathrm{C} 22$ & $-130.3(3)$ & $\mathrm{O} 31-\mathrm{C} 31-\mathrm{C} 32-\mathrm{C} 13$ & $-29.9(3)$ \\
$\mathrm{C} 3-\mathrm{N} 4-\mathrm{C} 21-\mathrm{C} 26$ & $-175.9(3)$ & $\mathrm{O} 32-\mathrm{C} 31-\mathrm{C} 32-\mathrm{Cl}$ & $151.5(3)$ \\
$\mathrm{C} 5-\mathrm{N} 4-\mathrm{C} 21-\mathrm{C} 26$ & $53.6(3)$ & $\mathrm{O} 31-\mathrm{C} 31-\mathrm{C} 32-\mathrm{C} 2$ & $91.3(3)$ \\
$\mathrm{C} 26-\mathrm{C} 21-\mathrm{C} 22-\mathrm{C} 23$ & $2.8(5)$ & $\mathrm{O} 32-\mathrm{C} 31-\mathrm{C} 32-\mathrm{C} 2$ & $-87.3(3)$ \\
$\mathrm{N} 4-\mathrm{C} 21-\mathrm{C} 22-\mathrm{C} 23$ & $-173.3(3)$ & $\mathrm{O} 31-\mathrm{C} 31-\mathrm{C} 32-\mathrm{C} 11$ & $-150.6(2)$ \\
$\mathrm{C} 21-\mathrm{C} 22-\mathrm{C} 23-\mathrm{C} 24$ & $-0.7(5)$ & $\mathrm{O} 32-\mathrm{C} 31-\mathrm{C} 32-\mathrm{C} 11$ & $30.8(4)$ \\
\hline
\end{tabular}

Hydrogen-bond geometry $\left(\AA,{ }^{\circ}\right)$

\begin{tabular}{lllll}
\hline$D-\mathrm{H} \cdots A$ & $D-\mathrm{H}$ & $\mathrm{H} \cdots A$ & $D \cdots A$ & $D-\mathrm{H} \cdots A$ \\
\hline $\mathrm{N} 1-\mathrm{H} 11 \cdots \mathrm{O} 31$ & $0.92(4)$ & $1.86(4)$ & $2.775(4)$ & $172(3)$ \\
$\mathrm{N} 1-\mathrm{H} 12 \cdots \mathrm{O} 32^{\mathrm{i}}$ & $0.97(3)$ & $1.80(3)$ & $2.724(3)$ & $158(3)$ \\
\hline
\end{tabular}

Symmetry code: (i) $x-1 / 2,-y+1, z$.

Bis(4-(4-methoxyphenyl)piperazin-1-ium) chloranilate(2-) dihydrate (XII)

Crystal data

$\mathrm{C}_{11} \mathrm{H}_{17} \mathrm{~N}_{2} \mathrm{O}^{+} \cdot 0.5 \mathrm{C}_{6} \mathrm{Cl}_{2} \mathrm{O}_{4}{ }^{2-} \cdot \mathrm{H}_{2} \mathrm{O}$

$M_{r}=314.76$

Monoclinic, $P 2_{1} / n$

$a=9.1597(5) \AA$

$b=15.1434(8) \AA$

$c=10.8742(6) \AA$

$\beta=102.067(5)^{\circ}$

$V=1475.02(14) \AA^{3}$

$Z=4$

\section{Data collection}

Oxford Diffraction Xcalibur with Sapphire CCD diffractometer

Radiation source: Enhance (Mo) X-ray Source

Graphite monochromator

$\omega$ scans

Absorption correction: multi-scan

(CrysAlis RED; Oxford Diffraction, 2009)

$T_{\min }=0.892, T_{\max }=0.947$

\section{Refinement}

Refinement on $F^{2}$

Least-squares matrix: full

$R\left[F^{2}>2 \sigma\left(F^{2}\right)\right]=0.039$
$F(000)=664$

$D_{\mathrm{x}}=1.417 \mathrm{Mg} \mathrm{m}^{-3}$

Mo $K \alpha$ radiation, $\lambda=0.71073 \AA$

Cell parameters from 3253 reflections

$\theta=2.6-28.0^{\circ}$

$\mu=0.28 \mathrm{~mm}^{-1}$

$T=296 \mathrm{~K}$

Block, colourless

$0.44 \times 0.24 \times 0.20 \mathrm{~mm}$

9650 measured reflections 9650 independent reflections

7444 reflections with $I>2 \sigma(I)$

$\theta_{\max }=27.6^{\circ}, \theta_{\min }=2.6^{\circ}$

$h=-11 \rightarrow 11$

$k=-19 \rightarrow 19$

$l=-13 \rightarrow 14$

$w R\left(F^{2}\right)=0.105$

$S=1.02$

9650 reflections 
204 parameters

0 restraints

Hydrogen site location: mixed

$\mathrm{H}$ atoms treated by a mixture of independent and constrained refinement

$$
\begin{aligned}
& w=1 /\left[\sigma^{2}\left(F_{\mathrm{o}}^{2}\right)+(0.0573 P)^{2}+0.263 P\right] \\
& \text { where } P=\left(F_{\mathrm{o}}^{2}+2 F_{\mathrm{c}}^{2}\right) / 3 \\
& (\Delta / \sigma)_{\max }<0.001 \\
& \Delta \rho_{\max }=0.23 \mathrm{e} \AA^{-3} \\
& \Delta \rho_{\min }=-0.32 \mathrm{e} \AA^{-3}
\end{aligned}
$$

Special details

Experimental. Compound (XII). IR ( $\left.\mathrm{KBr}, \mathrm{cm}^{-1}\right) 3311(\mathrm{OH}), 3073\left(\mathrm{NH}_{2}\right), 2825\left(\mathrm{OCH}_{3}\right), 1561(\mathrm{COO}), 793$ and $741(\mathrm{CCl})$ . NMR $\left.\left(\mathrm{CDCl}_{3}\right) \delta\left({ }^{1} \mathrm{H}\right)\right) 3.11\left(\mathrm{~m}, 4 \mathrm{H}\right.$, piperazine), $3.40(\mathrm{~m}, 4 \mathrm{H}$, piperazine $), 3.77\left(\mathrm{~s}, 3 \mathrm{H}, \mathrm{OCH}_{3}\right), 6.88(\mathrm{~m}, 4 \mathrm{H}$, methoxyphenyl).

Geometry. All esds (except the esd in the dihedral angle between two 1.s. planes) are estimated using the full covariance matrix. The cell esds are taken into account individually in the estimation of esds in distances, angles and torsion angles;

\begin{tabular}{|c|c|c|c|c|}
\hline & $x$ & $y$ & $z$ & $U_{\text {iso }} * / U_{\text {eq }}$ \\
\hline N1 & $0.4381(2)$ & $0.32824(14)$ & $0.35576(19)$ & $0.0420(5)$ \\
\hline H11 & $0.437(2)$ & $0.3477(15)$ & $0.278(3)$ & $0.050^{*}$ \\
\hline H12 & $0.420(3)$ & $0.3725(16)$ & $0.405(2)$ & $0.050 *$ \\
\hline $\mathrm{C} 2$ & $0.5890(2)$ & $0.29024(17)$ & $0.4015(2)$ & $0.0472(6)$ \\
\hline $\mathrm{H} 2 \mathrm{~A}$ & 0.6629 & 0.3370 & 0.4125 & $0.057 *$ \\
\hline $\mathrm{H} 2 \mathrm{~B}$ & 0.6115 & 0.2490 & 0.3397 & $0.057 *$ \\
\hline $\mathrm{C} 3$ & $0.5965(2)$ & $0.24346(16)$ & $0.5241(2)$ & $0.0427(6)$ \\
\hline $\mathrm{H} 3 \mathrm{~A}$ & 0.6939 & 0.2164 & 0.5509 & $0.051 *$ \\
\hline H3B & 0.5827 & 0.2856 & 0.5878 & $0.051^{*}$ \\
\hline N4 & 0.48097 (19) & $0.17569(13)$ & $0.51047(18)$ & $0.0398(5)$ \\
\hline C5 & $0.3334(2)$ & $0.21513(17)$ & $0.4745(2)$ & $0.0455(6)$ \\
\hline $\mathrm{H} 5 \mathrm{~A}$ & 0.3186 & 0.2572 & 0.5381 & $0.055^{*}$ \\
\hline H5B & 0.2576 & 0.1696 & 0.4681 & $0.055^{*}$ \\
\hline C6 & $0.3188(2)$ & $0.26139(16)$ & $0.3494(2)$ & $0.0454(6)$ \\
\hline H6A & 0.3256 & 0.2184 & 0.2847 & $0.054 *$ \\
\hline H6B & 0.2221 & 0.2899 & 0.3271 & $0.054^{*}$ \\
\hline $\mathrm{C} 21$ & $0.5025(2)$ & $0.10929(15)$ & $0.6041(2)$ & $0.0357(5)$ \\
\hline $\mathrm{C} 22$ & $0.6331(2)$ & $0.05976(16)$ & $0.6228(2)$ & $0.0432(6)$ \\
\hline $\mathrm{H} 22$ & 0.7033 & 0.0719 & 0.5744 & $0.052 *$ \\
\hline $\mathrm{C} 23$ & $0.6610(2)$ & $-0.00626(16)$ & $0.7103(2)$ & $0.0457(6)$ \\
\hline $\mathrm{H} 23$ & 0.7503 & -0.0375 & 0.7222 & $0.055^{*}$ \\
\hline $\mathrm{C} 24$ & $0.5565(2)$ & $-0.02672(15)$ & $0.7812(2)$ & $0.0418(6)$ \\
\hline $\mathrm{C} 25$ & $0.4243(2)$ & $0.01896(16)$ & $0.7614(2)$ & $0.0452(6)$ \\
\hline $\mathrm{H} 25$ & 0.3516 & 0.0040 & 0.8059 & $0.054^{*}$ \\
\hline $\mathrm{C} 26$ & $0.3991(2)$ & $0.08760(16)$ & $0.6747(2)$ & $0.0419(6)$ \\
\hline $\mathrm{H} 26$ & 0.3106 & 0.1195 & 0.6641 & $0.050 *$ \\
\hline $\mathrm{O} 24$ & $0.59700(19)$ & $-0.09318(12)$ & 0.86827 (19) & $0.0615(5)$ \\
\hline $\mathrm{C} 27$ & $0.4842(3)$ & $-0.12833(17)$ & $0.9257(3)$ & $0.0577(7)$ \\
\hline $\mathrm{H} 27 \mathrm{~A}$ & 0.5208 & -0.1806 & 0.9720 & $0.086 *$ \\
\hline $\mathrm{H} 27 \mathrm{~B}$ & 0.3980 & -0.1429 & 0.8621 & $0.086^{*}$ \\
\hline
\end{tabular}
correlations between esds in cell parameters are only used when they are defined by crystal symmetry. An approximate (isotropic) treatment of cell esds is used for estimating esds involving l.s. planes.

Refinement. Refined as a 2-component twin.

Fractional atomic coordinates and isotropic or equivalent isotropic displacement parameters $\left(\AA^{2}\right)$ 


$\begin{array}{lllll}\mathrm{H} 27 \mathrm{C} & 0.4573 & -0.0853 & 0.9820 & 0.086^{*} \\ \mathrm{C} 31 & 0.4846(2) & 0.41334(14) & 0.0515(2) & 0.0301(5) \\ \mathrm{O} 31 & 0.46952(17) & 0.34242(10) & 0.10569(14) & 0.0429(4) \\ \mathrm{C} 32 & 0.4674(2) & 0.42353(14) & -0.0785(2) & 0.0313(5) \\ \text { C132 } & 0.42518(7) & 0.33070(4) & -0.17371(6) & 0.04725(19) \\ \text { O33 } & 0.54043(16) & 0.48217(10) & 0.25106(14) & 0.0419(4) \\ \text { C33 } & 0.5224(2) & 0.49523(14) & 0.13592(19) & 0.0297(5) \\ \text { O41 } & 0.3458(2) & 0.45297(16) & 0.5134(2) & 0.0698(6) \\ \text { H41 } & 0.383(4) & 0.464(2) & 0.589(4) & 0.105^{*} \\ \text { H42 } & 0.295(4) & 0.497(2) & 0.492(3) & 0.105^{*}\end{array}$

Atomic displacement parameters $\left(\AA^{2}\right)$

\begin{tabular}{lllllll}
\hline & $U^{11}$ & $U^{22}$ & $U^{33}$ & $U^{12}$ & $U^{13}$ & $U^{23}$ \\
\hline $\mathrm{N} 1$ & $0.0531(12)$ & $0.0465(13)$ & $0.0271(11)$ & $0.0008(11)$ & $0.0101(9)$ & $0.0103(10)$ \\
$\mathrm{C} 2$ & $0.0458(13)$ & $0.0526(15)$ & $0.0447(15)$ & $-0.0023(12)$ & $0.0132(11)$ & $0.0145(13)$ \\
$\mathrm{C} 3$ & $0.0397(12)$ & $0.0497(14)$ & $0.0362(14)$ & $-0.0084(12)$ & $0.0026(10)$ & $0.0099(12)$ \\
$\mathrm{N} 4$ & $0.0358(10)$ & $0.0461(11)$ & $0.0344(11)$ & $-0.0073(9)$ & $0.0003(8)$ & $0.0123(9)$ \\
$\mathrm{C} 5$ & $0.0372(12)$ & $0.0559(16)$ & $0.0411(14)$ & $-0.0077(12)$ & $0.0028(10)$ & $0.0152(13)$ \\
$\mathrm{C} 6$ & $0.0462(13)$ & $0.0509(15)$ & $0.0343(14)$ & $-0.0037(12)$ & $-0.0026(10)$ & $0.0082(12)$ \\
$\mathrm{C} 21$ & $0.0368(12)$ & $0.0404(13)$ & $0.0280(12)$ & $-0.0096(11)$ & $0.0027(9)$ & $0.0032(10)$ \\
C22 & $0.0374(13)$ & $0.0471(15)$ & $0.0472(15)$ & $-0.0058(11)$ & $0.0139(11)$ & $0.0057(12)$ \\
C23 & $0.0358(12)$ & $0.0451(14)$ & $0.0566(17)$ & $0.0018(11)$ & $0.0103(11)$ & $0.0088(13)$ \\
C24 & $0.0462(13)$ & $0.0399(14)$ & $0.0375(14)$ & $-0.0005(12)$ & $0.0043(10)$ & $0.0093(11)$ \\
C25 & $0.0429(13)$ & $0.0540(16)$ & $0.0413(15)$ & $-0.0008(12)$ & $0.0146(10)$ & $0.0123(12)$ \\
C26 & $0.0358(12)$ & $0.0498(15)$ & $0.0398(14)$ & $0.0045(11)$ & $0.0070(10)$ & $0.0125(12)$ \\
O24 & $0.0567(11)$ & $0.0620(12)$ & $0.0673(14)$ & $0.0079(9)$ & $0.0164(9)$ & $0.0339(11)$ \\
C27 & $0.0752(18)$ & $0.0480(16)$ & $0.0522(18)$ & $-0.0012(15)$ & $0.0190(14)$ & $0.0151(14)$ \\
C31 & $0.0292(10)$ & $0.0331(12)$ & $0.0290(12)$ & $0.0005(9)$ & $0.0083(8)$ & $0.0032(10)$ \\
O31 & $0.0612(10)$ & $0.0355(9)$ & $0.0327(9)$ & $-0.0024(8)$ & $0.0115(7)$ & $0.0066(7)$ \\
C32 & $0.0368(11)$ & $0.0315(11)$ & $0.0256(11)$ & $-0.0004(10)$ & $0.0060(8)$ & $-0.0010(9)$ \\
C132 & $0.0648(4)$ & $0.0407(3)$ & $0.0347(3)$ & $-0.0058(3)$ & $0.0068(3)$ & $-0.0065(3)$ \\
O33 & $0.0582(9)$ & $0.0456(10)$ & $0.0215(8)$ & $-0.0073(8)$ & $0.0073(7)$ & $0.0036(7)$ \\
C33 & $0.0282(10)$ & $0.0381(12)$ & $0.0232(11)$ & $0.0019(9)$ & $0.0065(8)$ & $0.0014(9)$ \\
O41 & $0.0876(16)$ & $0.0781(15)$ & $0.0408(12)$ & $0.0001(12)$ & $0.0066(10)$ & $-0.0146(12)$ \\
& & & & & & \\
\hline
\end{tabular}

Geometric parameters $\left(\AA,{ }^{\circ}\right)$

\begin{tabular}{llll}
\hline $\mathrm{N} 1-\mathrm{C} 6$ & $1.481(3)$ & $\mathrm{C} 23-\mathrm{C} 24$ & $1.384(3)$ \\
$\mathrm{N} 1-\mathrm{C} 2$ & $1.484(3)$ & $\mathrm{C} 23-\mathrm{H} 23$ & 0.9300 \\
$\mathrm{~N} 1-\mathrm{H} 11$ & $0.89(3)$ & $\mathrm{C} 24-\mathrm{C} 25$ & $1.372(3)$ \\
$\mathrm{N} 1-\mathrm{H} 12$ & $0.89(2)$ & $\mathrm{C} 24-\mathrm{O} 24$ & $1.379(3)$ \\
$\mathrm{C} 2-\mathrm{C} 3$ & $1.498(3)$ & $\mathrm{C} 25-\mathrm{C} 26$ & $1.390(3)$ \\
$\mathrm{C} 2-\mathrm{H} 2 \mathrm{~A}$ & 0.9700 & $\mathrm{C} 25-\mathrm{H} 25$ & 0.9300 \\
$\mathrm{C} 2-\mathrm{H} 2 \mathrm{~B}$ & 0.9700 & $\mathrm{C} 26-\mathrm{H} 26$ & 0.9300 \\
$\mathrm{C} 3-\mathrm{N} 4$ & $1.459(3)$ & $\mathrm{O} 24-\mathrm{C} 27$ & $1.419(3)$ \\
$\mathrm{C} 3-\mathrm{H} 3 \mathrm{~A}$ & 0.9700 & $\mathrm{C} 27-\mathrm{H} 27 \mathrm{~A}$ & 0.9600 \\
$\mathrm{C} 3-\mathrm{H} 3 \mathrm{~B}$ & 0.9700 & $\mathrm{C} 27-\mathrm{H} 27 \mathrm{~B}$ & 0.9600
\end{tabular}




\begin{tabular}{|c|c|c|c|}
\hline $\mathrm{N} 4-\mathrm{C} 21$ & $1.415(3)$ & $\mathrm{C} 27-\mathrm{H} 27 \mathrm{C}$ & 0.9600 \\
\hline $\mathrm{N} 4-\mathrm{C} 5$ & $1.455(3)$ & $\mathrm{C} 31-\mathrm{O} 31$ & $1.246(2)$ \\
\hline $\mathrm{C} 5-\mathrm{C} 6$ & $1.511(3)$ & $\mathrm{C} 31-\mathrm{C} 32$ & $1.398(3)$ \\
\hline $\mathrm{C} 5-\mathrm{H} 5 \mathrm{~A}$ & 0.9700 & $\mathrm{C} 31-\mathrm{C} 33$ & $1.539(3)$ \\
\hline $\mathrm{C} 5-\mathrm{H} 5 \mathrm{~B}$ & 0.9700 & $\mathrm{C} 32-\mathrm{C} 33^{\mathrm{i}}$ & $1.392(3)$ \\
\hline $\mathrm{C} 6-\mathrm{H} 6 \mathrm{~A}$ & 0.9700 & $\mathrm{C} 32-\mathrm{Cl} 32$ & $1.741(2)$ \\
\hline $\mathrm{C} 6-\mathrm{H} 6 \mathrm{~B}$ & 0.9700 & $\mathrm{O} 33-\mathrm{C} 33$ & $1.244(2)$ \\
\hline $\mathrm{C} 21-\mathrm{C} 26$ & $1.378(3)$ & $\mathrm{C} 33-\mathrm{C} 32^{\mathrm{i}}$ & $1.392(3)$ \\
\hline $\mathrm{C} 21-\mathrm{C} 22$ & $1.390(3)$ & $\mathrm{O} 41-\mathrm{H} 41$ & $0.83(4)$ \\
\hline $\mathrm{C} 22-\mathrm{C} 23$ & $1.367(3)$ & $\mathrm{O} 41-\mathrm{H} 42$ & $0.82(3)$ \\
\hline $\mathrm{C} 22-\mathrm{H} 22$ & 0.9300 & & \\
\hline $\mathrm{C} 6-\mathrm{N} 1-\mathrm{C} 2$ & $112.16(18)$ & $\mathrm{C} 26-\mathrm{C} 21-\mathrm{N} 4$ & $124.1(2)$ \\
\hline $\mathrm{C} 6-\mathrm{N} 1-\mathrm{H} 11$ & $108.7(15)$ & $\mathrm{C} 22-\mathrm{C} 21-\mathrm{N} 4$ & $118.3(2)$ \\
\hline $\mathrm{C} 2-\mathrm{N} 1-\mathrm{H} 11$ & $105.7(14)$ & $\mathrm{C} 23-\mathrm{C} 22-\mathrm{C} 21$ & $121.7(2)$ \\
\hline $\mathrm{C} 6-\mathrm{N} 1-\mathrm{H} 12$ & $108.2(15)$ & $\mathrm{C} 23-\mathrm{C} 22-\mathrm{H} 22$ & 119.1 \\
\hline $\mathrm{C} 2-\mathrm{N} 1-\mathrm{H} 12$ & $111.7(16)$ & $\mathrm{C} 21-\mathrm{C} 22-\mathrm{H} 22$ & 119.1 \\
\hline $\mathrm{H} 11-\mathrm{N} 1-\mathrm{H} 12$ & $110(2)$ & $\mathrm{C} 22-\mathrm{C} 23-\mathrm{C} 24$ & $120.1(2)$ \\
\hline $\mathrm{N} 1-\mathrm{C} 2-\mathrm{C} 3$ & 110.34 (18) & $\mathrm{C} 22-\mathrm{C} 23-\mathrm{H} 23$ & 120.0 \\
\hline $\mathrm{N} 1-\mathrm{C} 2-\mathrm{H} 2 \mathrm{~A}$ & 109.6 & $\mathrm{C} 24-\mathrm{C} 23-\mathrm{H} 23$ & 120.0 \\
\hline $\mathrm{C} 3-\mathrm{C} 2-\mathrm{H} 2 \mathrm{~A}$ & 109.6 & $\mathrm{C} 25-\mathrm{C} 24-\mathrm{O} 24$ & $125.3(2)$ \\
\hline $\mathrm{N} 1-\mathrm{C} 2-\mathrm{H} 2 \mathrm{~B}$ & 109.6 & $\mathrm{C} 25-\mathrm{C} 24-\mathrm{C} 23$ & $119.4(2)$ \\
\hline $\mathrm{C} 3-\mathrm{C} 2-\mathrm{H} 2 \mathrm{~B}$ & 109.6 & $\mathrm{O} 24-\mathrm{C} 24-\mathrm{C} 23$ & $115.3(2)$ \\
\hline $\mathrm{H} 2 \mathrm{~A}-\mathrm{C} 2-\mathrm{H} 2 \mathrm{~B}$ & 108.1 & $\mathrm{C} 24-\mathrm{C} 25-\mathrm{C} 26$ & $120.0(2)$ \\
\hline $\mathrm{N} 4-\mathrm{C} 3-\mathrm{C} 2$ & $110.20(19)$ & $\mathrm{C} 24-\mathrm{C} 25-\mathrm{H} 25$ & 120.0 \\
\hline $\mathrm{N} 4-\mathrm{C} 3-\mathrm{H} 3 \mathrm{~A}$ & 109.6 & $\mathrm{C} 26-\mathrm{C} 25-\mathrm{H} 25$ & 120.0 \\
\hline $\mathrm{C} 2-\mathrm{C} 3-\mathrm{H} 3 \mathrm{~A}$ & 109.6 & $\mathrm{C} 21-\mathrm{C} 26-\mathrm{C} 25$ & $121.3(2)$ \\
\hline $\mathrm{N} 4-\mathrm{C} 3-\mathrm{H} 3 \mathrm{~B}$ & 109.6 & $\mathrm{C} 21-\mathrm{C} 26-\mathrm{H} 26$ & 119.4 \\
\hline $\mathrm{C} 2-\mathrm{C} 3-\mathrm{H} 3 \mathrm{~B}$ & 109.6 & $\mathrm{C} 25-\mathrm{C} 26-\mathrm{H} 26$ & 119.4 \\
\hline $\mathrm{H} 3 \mathrm{~A}-\mathrm{C} 3-\mathrm{H} 3 \mathrm{~B}$ & 108.1 & $\mathrm{C} 24-\mathrm{O} 24-\mathrm{C} 27$ & $117.40(19)$ \\
\hline $\mathrm{C} 21-\mathrm{N} 4-\mathrm{C} 5$ & $117.85(18)$ & $\mathrm{O} 24-\mathrm{C} 27-\mathrm{H} 27 \mathrm{~A}$ & 109.5 \\
\hline $\mathrm{C} 21-\mathrm{N} 4-\mathrm{C} 3$ & $115.96(17)$ & $\mathrm{O} 24-\mathrm{C} 27-\mathrm{H} 27 \mathrm{~B}$ & 109.5 \\
\hline $\mathrm{C} 5-\mathrm{N} 4-\mathrm{C} 3$ & $110.60(18)$ & $\mathrm{H} 27 \mathrm{~A}-\mathrm{C} 27-\mathrm{H} 27 \mathrm{~B}$ & 109.5 \\
\hline $\mathrm{N} 4-\mathrm{C} 5-\mathrm{C} 6$ & $109.54(18)$ & $\mathrm{O} 24-\mathrm{C} 27-\mathrm{H} 27 \mathrm{C}$ & 109.5 \\
\hline $\mathrm{N} 4-\mathrm{C} 5-\mathrm{H} 5 \mathrm{~A}$ & 109.8 & $\mathrm{H} 27 \mathrm{~A}-\mathrm{C} 27-\mathrm{H} 27 \mathrm{C}$ & 109.5 \\
\hline $\mathrm{C} 6-\mathrm{C} 5-\mathrm{H} 5 \mathrm{~A}$ & 109.8 & $\mathrm{H} 27 \mathrm{~B}-\mathrm{C} 27-\mathrm{H} 27 \mathrm{C}$ & 109.5 \\
\hline $\mathrm{N} 4-\mathrm{C} 5-\mathrm{H} 5 \mathrm{~B}$ & 109.8 & $\mathrm{O} 31-\mathrm{C} 31-\mathrm{C} 32$ & $125.0(2)$ \\
\hline $\mathrm{C} 6-\mathrm{C} 5-\mathrm{H} 5 \mathrm{~B}$ & 109.8 & $\mathrm{O} 31-\mathrm{C} 31-\mathrm{C} 33$ & $116.47(18)$ \\
\hline $\mathrm{H} 5 \mathrm{~A}-\mathrm{C} 5-\mathrm{H} 5 \mathrm{~B}$ & 108.2 & $\mathrm{C} 32-\mathrm{C} 31-\mathrm{C} 33$ & $118.55(18)$ \\
\hline $\mathrm{N} 1-\mathrm{C} 6-\mathrm{C} 5$ & $110.47(18)$ & $\mathrm{C} 31-\mathrm{C} 32-\mathrm{C} 33^{\mathrm{i}}$ & $123.2(2)$ \\
\hline $\mathrm{N} 1-\mathrm{C} 6-\mathrm{H} 6 \mathrm{~A}$ & 109.6 & $\mathrm{C} 31-\mathrm{C} 32-\mathrm{Cl} 32$ & $118.45(17)$ \\
\hline $\mathrm{C} 5-\mathrm{C} 6-\mathrm{H} 6 \mathrm{~A}$ & 109.6 & $\mathrm{C} 33^{\mathrm{i}}-\mathrm{C} 32-\mathrm{Cl} 32$ & $118.31(16)$ \\
\hline $\mathrm{N} 1-\mathrm{C} 6-\mathrm{H} 6 \mathrm{~B}$ & 109.6 & $\mathrm{O} 33-\mathrm{C} 33-\mathrm{C} 32^{\mathrm{i}}$ & $125.8(2)$ \\
\hline $\mathrm{C} 5-\mathrm{C} 6-\mathrm{H} 6 \mathrm{~B}$ & 109.6 & $\mathrm{O} 33-\mathrm{C} 33-\mathrm{C} 31$ & $115.99(19)$ \\
\hline $\mathrm{H} 6 \mathrm{~A}-\mathrm{C} 6-\mathrm{H} 6 \mathrm{~B}$ & 108.1 & $\mathrm{C} 32^{\mathrm{i}}-\mathrm{C} 33-\mathrm{C} 31$ & $118.20(17)$ \\
\hline $\mathrm{C} 26-\mathrm{C} 21-\mathrm{C} 22$ & $117.5(2)$ & $\mathrm{H} 41-\mathrm{O} 41-\mathrm{H} 42$ & $102(3)$ \\
\hline $\mathrm{C} 6-\mathrm{N} 1-\mathrm{C} 2-\mathrm{C} 3$ & $-53.5(3)$ & $\mathrm{C} 22-\mathrm{C} 23-\mathrm{C} 24-\mathrm{O} 24$ & $-178.8(2)$ \\
\hline
\end{tabular}




$\begin{array}{llll}\mathrm{N} 1-\mathrm{C} 2-\mathrm{C} 3-\mathrm{N} 4 & 56.5(3) & \mathrm{O} 24-\mathrm{C} 24-\mathrm{C} 25-\mathrm{C} 26 & 176.9(2) \\ \mathrm{C} 2-\mathrm{C} 3-\mathrm{N} 4-\mathrm{C} 21 & 161.1(2) & \mathrm{C} 23-\mathrm{C} 24-\mathrm{C} 25-\mathrm{C} 26 & -2.8(4) \\ \mathrm{C} 2-\mathrm{C} 3-\mathrm{N} 4-\mathrm{C} 5 & -61.3(3) & \mathrm{C} 22-\mathrm{C} 21-\mathrm{C} 26-\mathrm{C} 25 & 0.1(3) \\ \mathrm{C} 21-\mathrm{N} 4-\mathrm{C} 5-\mathrm{C} 6 & -162.3(2) & \mathrm{N} 4-\mathrm{C} 21-\mathrm{C} 26-\mathrm{C} 25 & 177.1(2) \\ \mathrm{C} 3-\mathrm{N} 4-\mathrm{C} 5-\mathrm{C} 6 & 61.0(3) & \mathrm{C} 24-\mathrm{C} 25-\mathrm{C} 26-\mathrm{C} 21 & 2.3(4) \\ \mathrm{C} 2-\mathrm{N} 1-\mathrm{C} 6-\mathrm{C} 5 & 53.7(3) & \mathrm{C} 25-\mathrm{C} 24-\mathrm{O} 24-\mathrm{C} 27 & 12.2(4) \\ \mathrm{N} 4-\mathrm{C} 5-\mathrm{C} 6-\mathrm{N} 1 & -56.7(3) & \mathrm{C} 23-\mathrm{C} 24-\mathrm{O} 24-\mathrm{C} 27 & -168.1(2) \\ \mathrm{C} 5-\mathrm{N} 4-\mathrm{C} 21-\mathrm{C} 26 & -8.7(3) & \mathrm{O} 31-\mathrm{C} 31-\mathrm{C} 32-\mathrm{C} 33^{\mathrm{i}} & 176.5(2) \\ \mathrm{C} 3-\mathrm{N} 4-\mathrm{C} 21-\mathrm{C} 26 & 125.7(2) & \mathrm{C} 33-\mathrm{C} 31-\mathrm{C} 32-\mathrm{C} 33^{\mathrm{i}} & -2.4(3) \\ \mathrm{C} 5-\mathrm{N} 4-\mathrm{C} 21-\mathrm{C} 22 & 168.2(2) & \mathrm{O} 31-\mathrm{C} 31-\mathrm{C} 32-\mathrm{C} 32 & -1.3(3) \\ \mathrm{C} 3-\mathrm{N} 4-\mathrm{C} 21-\mathrm{C} 22 & -57.4(3) & \mathrm{C} 33-\mathrm{C} 31-\mathrm{C} 32-\mathrm{C} 132 & 179.81(14) \\ \mathrm{C} 26-\mathrm{C} 21-\mathrm{C} 22-\mathrm{C} 23 & -2.0(3) & \mathrm{O} 31-\mathrm{C} 31-\mathrm{C} 33-\mathrm{O} 33 & 2.8(3) \\ \mathrm{N} 4-\mathrm{C} 21-\mathrm{C} 22-\mathrm{C} 23 & -179.1(2) & \mathrm{C} 32-\mathrm{C} 31-\mathrm{C} 33-\mathrm{O} 33 & -178.16(18) \\ \mathrm{C} 21-\mathrm{C} 22-\mathrm{C} 23-\mathrm{C} 24 & 1.5(4) & \mathrm{O} 31-\mathrm{C} 31-\mathrm{C} 33-\mathrm{C} 32^{\mathrm{i}} & -176.76(18) \\ \mathrm{C} 22-\mathrm{C} 23-\mathrm{C} 24-\mathrm{C} 25 & 0.9(4) & \mathrm{C} 32-\mathrm{C} 31-\mathrm{C} 33-\mathrm{C} 32^{\mathrm{i}} & 2.2(3) \\ \end{array}$

Symmetry code: (i) $-x+1,-y+1,-z$.

Hydrogen-bond geometry $\left(\AA,{ }^{\circ}\right)$

\begin{tabular}{lllll}
\hline$D-\mathrm{H} \cdots A$ & $D-\mathrm{H}$ & $\mathrm{H} \cdots A$ & $D \cdots A$ & $D-\mathrm{H} \cdots A$ \\
\hline $\mathrm{N} 1-\mathrm{H} 11 \cdots \mathrm{O} 31$ & $0.89(3)$ & $1.96(3)$ & $2.802(3)$ & $157(2)$ \\
$\mathrm{N} 1-\mathrm{H} 11 \cdots \mathrm{O} 33$ & $0.89(3)$ & $2.29(2)$ & $2.838(3)$ & $119(2)$ \\
$\mathrm{N} 1-\mathrm{H} 12 \cdots \mathrm{O} 41$ & $0.90(2)$ & $1.92(2)$ & $2.798(3)$ & $168(3)$ \\
$\mathrm{O} 41-\mathrm{H} 41 \cdots \mathrm{O} 33^{\mathrm{ii}}$ & $0.84(4)$ & $1.92(4)$ & $2.738(3)$ & $166(3)$ \\
$\mathrm{O} 41-\mathrm{H} 42 \cdots \mathrm{O} 24^{\text {iii }}$ & $0.82(3)$ & $2.49(3)$ & $3.269(3)$ & $160(3)$ \\
\hline
\end{tabular}

Symmetry codes: (ii) $-x+1,-y+1,-z+1$; (iii) $x-1 / 2,-y+1 / 2, z-1 / 2$. 